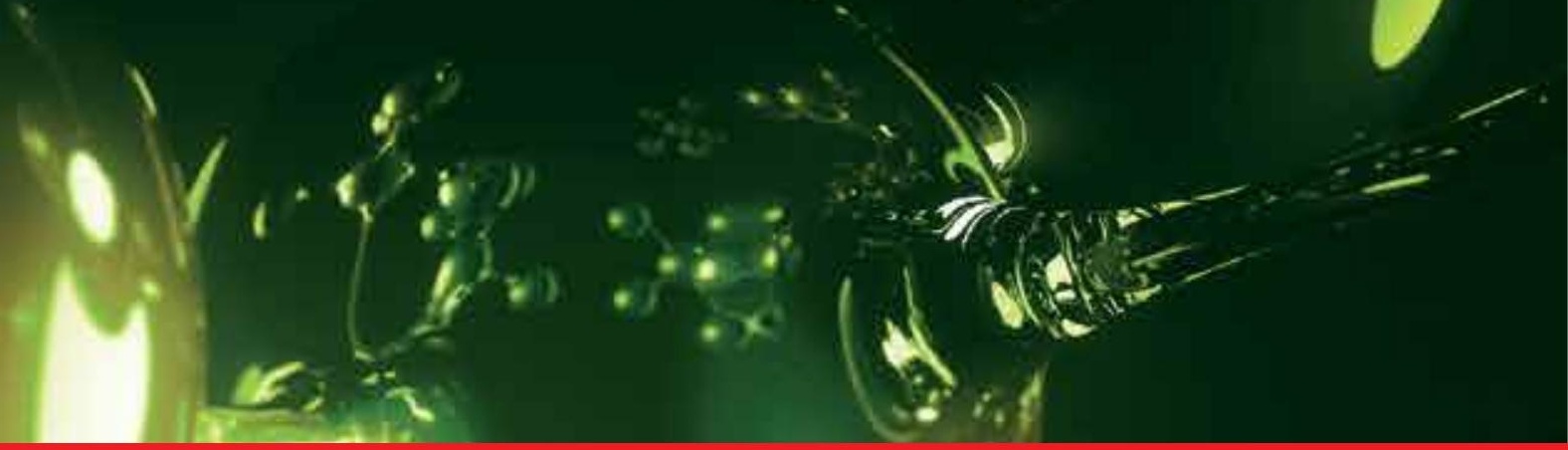

\title{
IntechOpen
}

\section{Modern Spectroscopic Techniques and Applications}

Edited by Maaz Khan, Gustavo Morari do Nascimento and Marwa El-Azazy

\section{D}





\section{Modern Spectroscopic Techniques and Applications}

Edited by Maaz Khan, Gustavo Morari do Nascimento and Marwa El-Azazy 

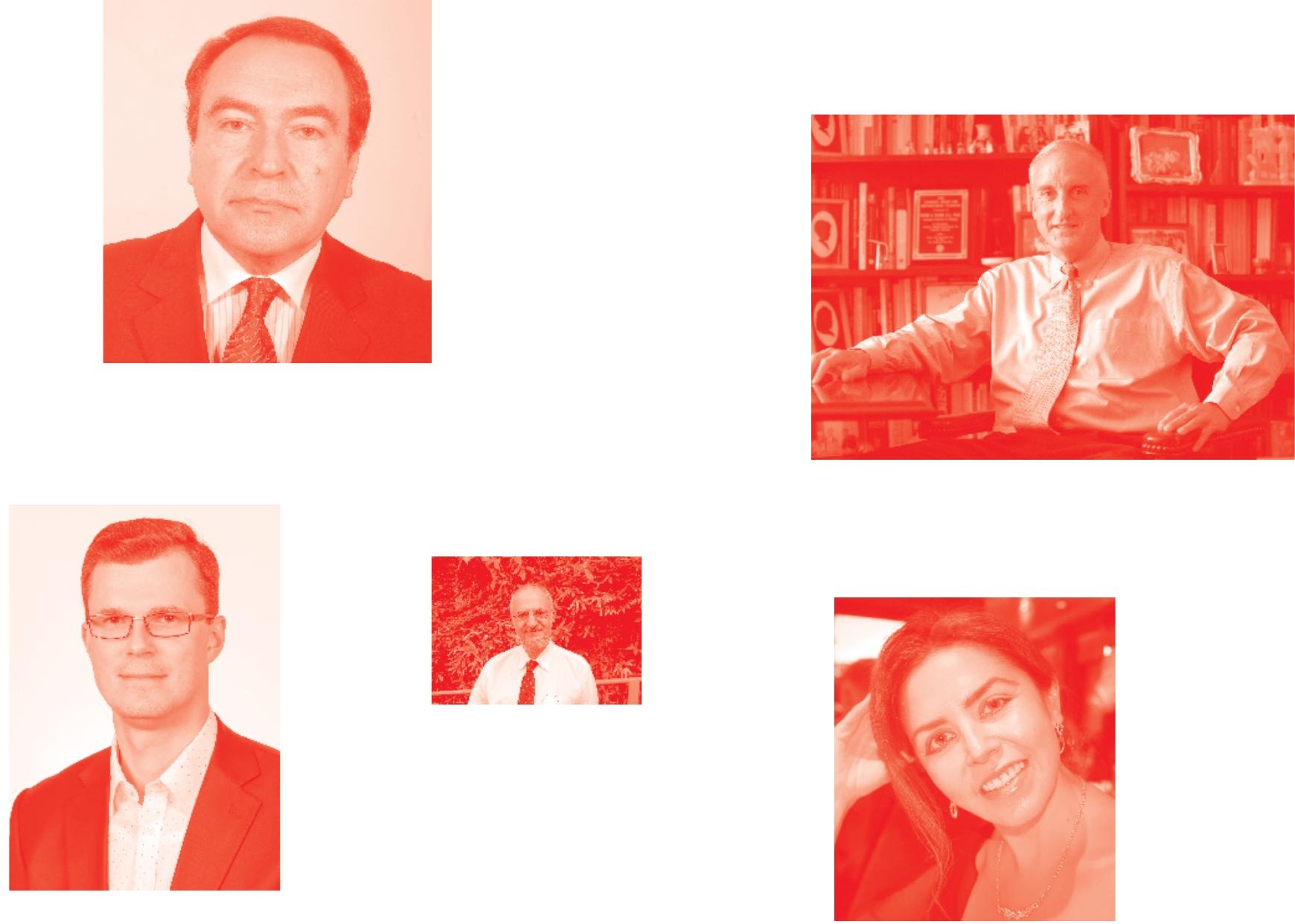

Supporting open minds since 2005
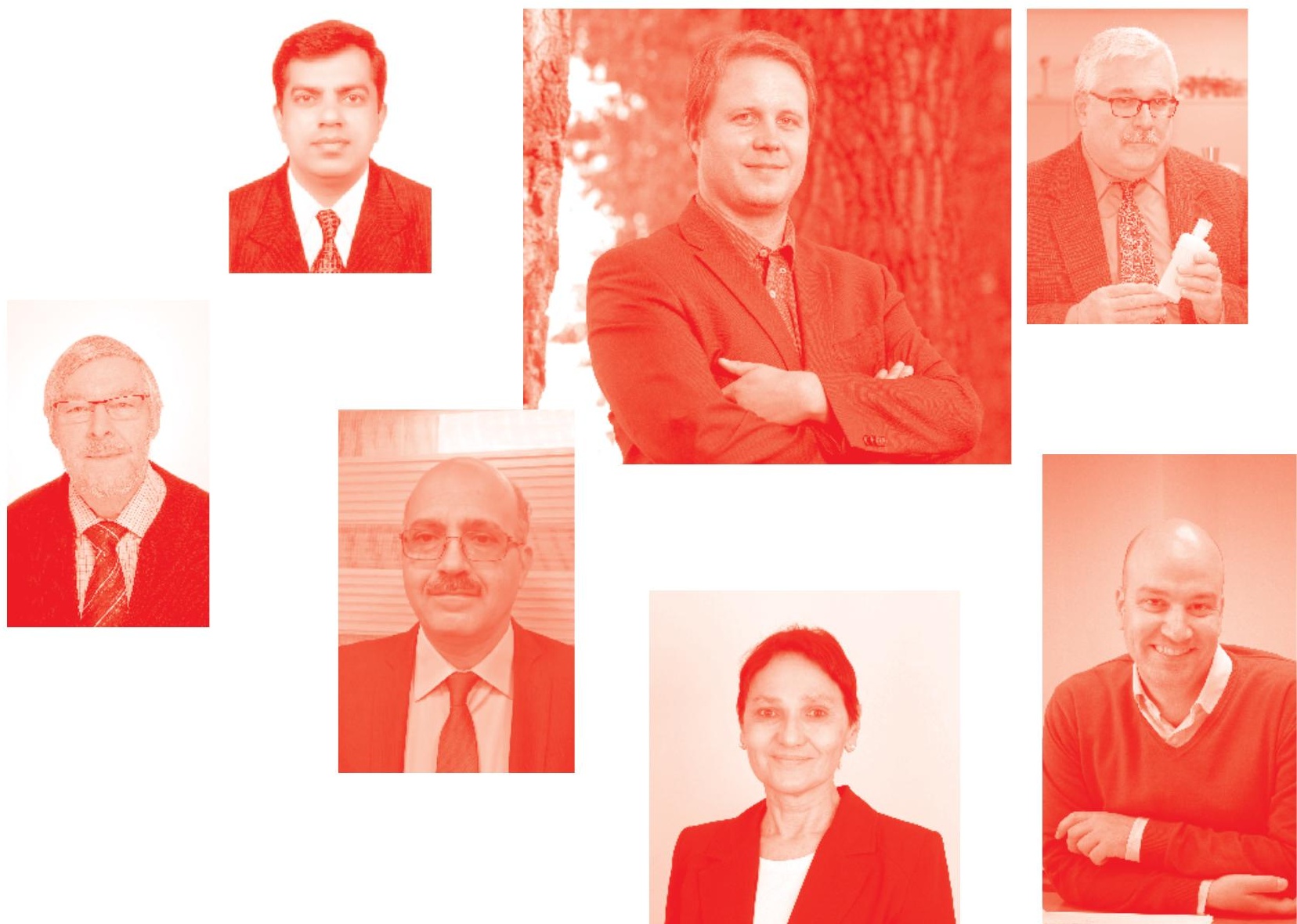
Modern Spectroscopic Techniques and Applications

http: //dx. doi. org/10.5772/intechopen. 77480

Edited by Maaz Khan, Gustavo Morari do Nascimento and Marwa El-Azazy

\section{Contributors}

Mahmoud Rahal, Abdeslam El Hajbi, Minerva Robles-Agudo, Martha Patricia Gonzalez Tejeda, Ignacio Rojas Rodríguez, Porfirio Essau Martinez Muñoz, Nathalie Dupuy, Catherine Rebufa, Fabien Girard, Jacques Artaud, Søren Hassing, Boukhlifi Fatima, Deniz Sahin, Gustavo Morari Do Nascimento

( ) The Editor(s) and the Author(s) 2020

The rights of the editor(s) and the author(s) have been asserted in accordance with the Copyright, Designs and Patents Act 1988. All rights to the book as a whole are reserved by INTECHOPEN LIMITED. The book as a whole (compilation) cannot be reproduced, distributed or used for commercial or non-commercial purposes without INTECHOPEN LIMITED's written permission. Enquiries concerning the use of the book should be directed to INTECHOPEN LIMITED rights and permissions department (permissions@intechopen.com).

Violations are liable to prosecution under the governing Copyright Law .

\section{(cc) BY}

Individual chapters of this publication are distributed under the terms of the Creative Commons Attribution 3.๑ Unported License which permits commercial use, distribution and reproduction of the individual chapters, provided the original author(s) and source publication are appropriately acknowledged. If so indicated, certain images may not be included under the Creative Commons license. In such cases users will need to obtain permission from the license holder to reproduce the material. More details and guidelines concerning content reuse and adaptation can be found at http : //www . intechopen . com/copyright-policy . html .

\section{Notice}

Statements and opinions expressed in the chapters are these of the individual contributors and not necessarily those of the editors or publisher. No responsibility is accepted for the accuracy of information contained in the published chapters. The publisher assumes no responsibility for any damage or injury to persons or property arising out of the use of any materials, instructions, methods or ideas contained in the book.

First published in London, United Kingdom, 2020 by IntechOpen IntechOpen is the global imprint of INTECHOPEN LIMITED, registered in England and Wales, registration number: 11086078 , 7th floor, 10 Lower Thames Street, London,

EC3R 6AF, United Kingdom

Printed in Croatia

British Library Cataloguing-in-Publication Data

A catalogue record for this book is available from the British Library

Additional hard and PDF copies can be obtained from orders@intechopen.com

Modern Spectroscopic Techniques and Applications

Edited by Maaz Khan, Gustavo Morari do Nascimento and Marwa El-Azazy

p. cm.

Print ISBN 978-1-78985-205-9

Online ISBN 978-1-78985-206-6

eBook (PDF) ISBN 978-1-78984-582-2 


\section{We are IntechOpen, \\ the world's leading publisher of Open Access books}

\section{Built by scientists, for scientists}

\section{$4,600+$}

Open access books available

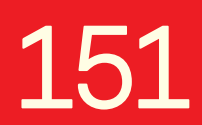

Countries delivered to

\section{$120,000+$}

International authors and editors

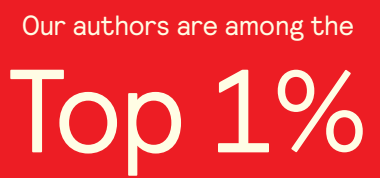

most cited scientists

Contributors from top 500 universities
$135 \mathrm{M}+$

Downloads
$12.2 \%$

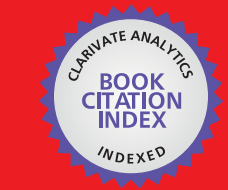

WEB OF SCIENCE ${ }^{\text {M }}$

Selection of our books indexed in the Book Citation Index in Web of Science ${ }^{\mathrm{TM}}$ Core Collection (BKCI)

\section{Interested in publishing with us? \\ Contact book.department@intechopen.com}

Numbers displayed above are based on latest data collected.

For more information visit www.intechopen.com 



\section{Meet the editors}

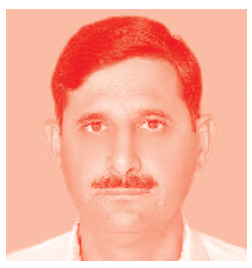

Dr Maaz Khan works as a Senior Researcher in PINSTECH, Pakistan. He has received his $\mathrm{PhD}$ and Post-Doctorate degree in material science. His research interests include fabrication of nanomaterials and their structural, optical, magnetic, and electrical characterizations. He has authored more than 100 research articles and published 8 books. Presently, he is serving as the Editor-in-Chief of "Journal of Materials, Processing and Design" and Executive Editor of "International Journal of Nano Studies \& Technology". He is also on the editorial board of several more journals in the material science field.

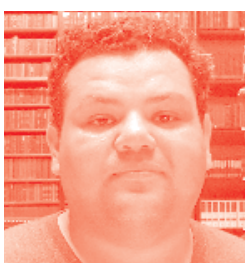

Dr Gustavo Morari do Nascimento is a professor at the Federal University of ABC. He has vast experience in many fields related to the characterization of nanomaterials using spectroscopic techniques associated to microscopic resolution. He obtained his doctoral degree from the University of São Paulo (USP) in 2004 with a thesis about the spectroscopic characterization of nanocomposites formed by conducting polymers and clays. Afterwards, he completed a post-doctoral degree at Massachusetts Institute of Technology (MIT) in the resonance Raman study of double-walled carbon nanotubes doped with halogens under the guidance of the legendary Mildred S. Dresselhaus. Back in Brazil, he spent three years (2009-2011) at the Federal University of Minas Gerais (UFMG) working on the synthesis of nanostructured carbon modified with molecular magnets. Nowadays, his research focus is on molecular characterization of modified carbon nanostructured materials and polymer nanocomposites using different spectroscopic techniques. Resonance Raman and SERS (surface enhanced Raman spectroscopy) coupled to microscopy techniques added to X-ray absorption techniques at National Synchrotron Light Laboratory have been the main techniques employed in the investigation.

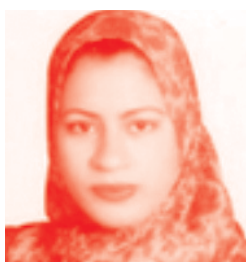

Dr Marwa El-Azazy is an Analytical Chemist and "experienced educator and researcher" with 20 years of teaching experience at several institutions. Her main research interest is the construction of sensors (using microfluidic platforms for point-of-caretesting of drugs, bio-analytes, and ion-selective electrodes), chemometrics and analytical method development, spectroscopic analyses of drugs and pharmaceuticals, synthesis and characterization of nanomaterials, and development of green chemistry approaches for wastewater treatment. Dr Marwa has a track record of research inputs including $>30$ refereed papers in prestigious international journals, several conference presentations, two book chapters, in addition to several research grants. She serves as a reviewer for a variety of international journals. 



\section{Contents}

Preface III

$\begin{array}{lr}\text { Chapter } 1 & 1\end{array}$

Atomic Spectroscopy

by Deniz Sahin

Chapter 2

What Is Vibrational Raman Spectroscopy: A Vibrational or an Electronic Spectroscopic Technique or Both?

by Søren Hassing

Chapter 3

Vibrational Zero-Point Energy of Organosilicon Compounds

by Mahmoud Rahal and Abdeslam El Hajbi

Chapter 4

Analysis of the Absorption Phenomenon through the Use of Finite Element Method

by Minerva Robles-Agudo, Martha Patricia Gonzalez-Tejeda,

Porfirio Esau Martinez-Muñoz and Ignacio Rojas-Rodríguez

Chapter 5

Discrimination by Infrared Spectroscopy: Application to Micronized

Locust Bean and Guar Gums

by Catherine Rébufa, Fabien Girard, Jacques Artaud and Nathalie Dupuy

Chapter 6

Raman Spectroscopy and Imaging of Carbon Allotropes

by Gustavo Morari do Nascimento

Chapter 7

Quantitative Analysis by IR: Determination of Chitin/Chitosan DD

by Boukhlifi Fatima 



\title{
Preface
}

Modern spectroscopy techniques are used for the analysis of materials, such as the elemental, molecular, and chemical compositions, atomic structure, perfection of surfaces of solids, surface layers, and phase boundaries. These techniques employ electromagnetic radiation that is typically classified by the wavelength region of the spectrum. Spectroscopy techniques are used as a tool for studying the structures of atoms and molecules, as a large number of wavelengths emitted by different materials makes it possible to investigate their structures in detail, including the electron configurations of the ground and various excited states.

The book is divided into seven chapters, which are mainly focused on the experimental as well as theoretical aspects. These chapters include an introduction to atomic spectroscopy, vibrational Raman spectroscopy, vibrational zero-point energy of organic compounds, analysis of light absorption phenomenon, and discrimination by infrared spectroscopy. All the authors are experts and active in their fields and therefore this book provides the latest information about these topics. The contents of the book provide fundamental knowledge necessary for the advanced study in the field of modern spectroscopic techniques and applications. Proper references have been provided at the end of each chapter, which can lead the readers to the best sources in the literature and help them read more detail about the topic.

I am indebted to all the authors for helping me to complete this project and also many thanks to the entire IntechOpen publishing team for making this project possible. Furthermore, I am thankful to Ms. Dolores Kuzelj, the Author Service Manager, for her pleasant and cooperative behavior during the publishing process of this book. I hope that this book will help the readers acquire further knowledge about modern spectroscopic techniques and their applications in different areas.

\author{
Maaz Khan \\ PINSTECH, \\ Islamabad, Pakistan
}

Gustavo Morari do Nascimento

Federal University of ABC,

Santo André, Brazil

Marwa El-Azazy

Department of Chemistry and Earth Sciences,

Qatar University,

Doha, Qatar 



\title{
Chapter 1
}

\section{Atomic Spectroscopy}

\author{
Deniz Sahin
}

\begin{abstract}
Atomic spectroscopy includes a number of analytical techniques used to determine the elemental composition of a sample (it can be gas, liquid, or solid) by observing its electromagnetic spectrum or its mass spectrum. Element concentrations of a millionth (ppm) or one billionth part (ppb) of the sample can be detected. There are different variations of atomic spectroscopy, emission, absorption, fluorescence, and mass spectroscopy. Determination of an appropriate technique requires a basic understanding of each technique since each has its individual strengths and limitations. This chapter is designed to provide a basic overview to the atomic spectroscopy techniques and how can you select the one that best suits our analytical problems.
\end{abstract}

Keywords: atomic absorption spectroscopy (AAS), atomic emission spectroscopy (AES), atomic fluorescence spectroscopy (AFS), X-ray fluorescence (XRF), mass spectroscopy (MS)

\section{Introduction}

Spectroscopy is the study of interactions between matter and different forms of electromagnetic radiation; when practiced to quantitative analysis, the term spectrometry is used.

Atomic spectroscopy includes the techniques of atomic absorption spectroscopy (AAS), atomic emission spectroscopy (AES), atomic fluorescence spectroscopy (AFS), X-ray fluorescence (XRF), and inorganic mass spectroscopy (MS). AAS, AES, and AFS exploit interactions between UV-visible light and the valence electrons of free gaseous atoms. In XRF, high-energy charged particles collide with inner-shell electrons of atom, initiating transitions with eventual emission of X-ray photons. For inorganic MS, ionized analyte atoms are separated in a magnetic field according to their mass to charge $(\mathrm{m} / \mathrm{z})$ ratio [1].

\section{Atomic spectroscopy: general principles}

Every element has a characteristic atomic structure, with a small, positively charged nucleus surrounded by a sufficient number of electrons necessary to maintain neutrality. Electrons settle into orbitals within an atom and one of the electrons can also jump from one energy level to the higher level by acquiring the necessitated energy (Figure 1). This energy is provided by colliding with other atoms, such as heating-AES, photons derived from light-AAS and AFS, or high-energy electronsXRF. Possible transitions happen, when the required energy reaches to the difference between two energy states $(\Delta \mathrm{E})$. A neutral atom may exist at a low energy shell 

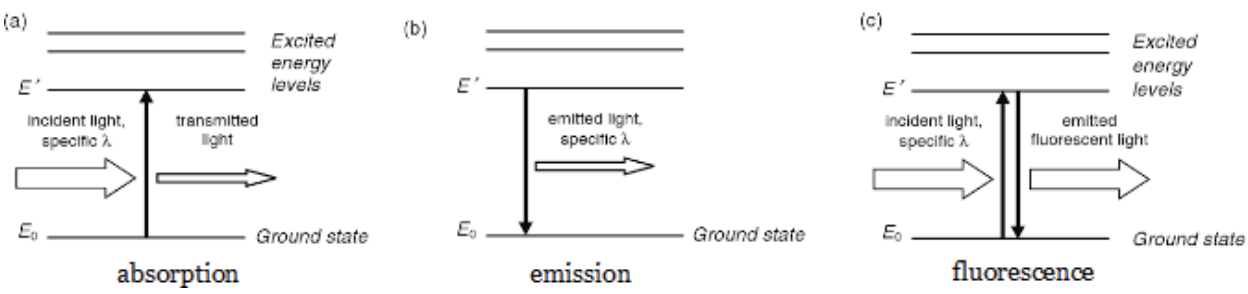

Figure 1.

Energy level diagrams to show transitions associated with (a) AAS, (b) AES, and (c) AFS. The vertical arrows indicate absorption or emission of light.

or ground state $\left(E_{0}\right)$, or at any of a group of excited states depending on how many electrons have been jumped to higher energy levels $\left(E^{\prime},\right)$ although it is normal to think for the first transition. Each element has a unique energy level and the $\Delta \mathrm{Es}$ associated with transitions between those levels.

The $\Delta \mathrm{E}$ for movements of valence electrons in most elements meets the energy equal to UV/visible radiation. The energy of a photon (E) is computed with the following equation:

$$
\mathrm{E}=\mathrm{ho}
$$

where $\mathrm{h}$ is Planck's constant $\left(6.63 \times 10^{-34} \mathrm{Js}\right)$ and $v$ the frequency of the waveform corresponding to that photon. The relationship between wavelength and frequency is showed by the equation below:

$$
v=\frac{c}{\lambda}
$$

where $\mathrm{c}$ is the speed of light and $\lambda$ the wavelength. Thus,

$$
\mathrm{E}=\frac{\mathrm{hc}}{\lambda}
$$

and a specific transition, $\Delta \mathrm{E}$, is associated with a unique wavelength.

When light of a specific wavelength enters an analytical system, outer shell electrons of the corresponding atoms will be excited as energy is absorbed. As a result, the amount of light transmitted from the system to detector will be reduced, this is understood as AAS (Figure 1a).

Under appropriate circumstances, outer shell electrons of vaporized atoms may be excited by heating. As these electrons return to the more stable ground state, energy is lost. As Figure 1b shows, some of this energy is emitted as light, which can be measured with a detector, this is AES.

Some of the radiant energy absorbed by ground state atoms can be emitted as light as the atom returns to the ground state i.e. AFS (Figure 1c).

When high-energy photons strike to a massive particle, it can excite an inner shell electron of the atom. The forming inner orbital vacancy can be filled with an outer shell electron. The transition is created by an emission of an X-Ray photon. This process is called X-ray fluorescence (XRF) [2-6].

The energy of the emission i.e. the wavelength is characteristic of the atom (element) from which it originated while the intensity of the emission is related to concentration of the atoms in the sample [7].

The high temperature inductively coupled plasma has been successfully used as an effective ion source for a mass spectroscopy, the type of method of inductively coupled plasma-mass spectroscopy (ICP-MS) is routinely used for measurements of trace elements in clinical and biological samples $[8,9]$. 
It follows from Eqs. 1-3 that the wavelengths of the absorbed or emitted light are unique to a given element.

\section{Atomic spectroscopy: instrumentation}

Formation of the atomic vapor i.e. atomization is the major principle of emission, absorption, and fluorescence techniques. The most critical component of instruments used in atomic spectroscopy is the atomization sources and sample introduction devices with an associated spectrometer for wavelength selection and detection of light. Atomization involves the several key (the basic) steps: solvent removal, separation from anion and other elements of the matrix, and reduction of ions to the ground state atom. The design of an AFS instrument is similar to those for AAS and AES except that the light source and the detector are located at a right angle (Figure 2).

A light source which emits the sharp atomic lines of the element to be determined is selected. There are two types of light sources used in these instruments: continuous sources and line sources. A continuous source, also called to as a broad-band source, emits radiation over a broad range of wavelengths. A line source, on the other hand, emits radiation at specific wavelengths, but this source of radiation is not as pure as radiation from a laser. Table 1 provides a list of most common kinds of lamps considered to be light sources.

The atomizer is any device which will produce ground state atoms as a vapor into the light path. Many atomizers utilized for AFS are similar to those used for AAS and AES. The atomizers most commonly used in these techniques are flames and electrothermal atomizers [10]. The flame provides for easy and fast measurements with few interferences and is preferred at any appropriate concentration for the analyte. Flame atomizers contain a pneumatic nebulizer, an expansion chamber, and an air-acetylene laminar flame with a $10 \mathrm{~cm}$ path length. The typical pneumatic nebulizer for sample introduction is insufficient, and although elements such as $\mathrm{Na}$ and $\mathrm{K}$ can be determined in biological samples by flame AES, flame atomization is more suitable for AAS and AFS. AAS measurements can detect concentrations at approximately $1 \mu \mathrm{g} / \mathrm{ml}(\mathrm{ppm})$ or more. Devices are being developed to overcome these limitations of the typical nebulizer. Atomization can be reached to $100 \%$ and the devices can also generate the sample as a pulse flow rather than the continuous flow. Most systems use a graphite tube which is heated electrical energy, a technique called graphite furnace atomization, although other materials are sometimes employed. A programmed sequence of the furnace temperature is used in electrically heated graphite tube. With this atomizer, $10-50 \mu \mathrm{l}$ of test solution is dried, organic material is destroyed, and the analyte ions dissociated from anions for reduction to ground state atoms. This atomizer also produces temperatures up to $3000 \mathrm{~K}$ which allows to form an atomic vapor of refractory elements such as aluminum and chromium. Since the analyte is atomized and retained within a small volume furnace, this procures a dense atom population. The technique is extremely sensitive as it allows one to detect a few $\mu \mathrm{g} / \mathrm{ml}$ concentrations of the analyte. Although the technique is widely used for AAS, electrothermal atomization will

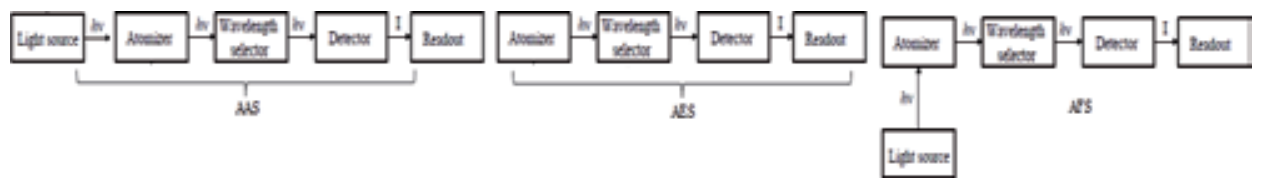

Figure 2.

Schematic diagram of an AAS, AES, and AFS instrument. 


\begin{tabular}{|c|c|c|}
\hline stourus & Mavederall heaul & Uesiul fex... \\
\hline$H_{1}$ and Dalate & cerrn.um su.roc tian $160590 \mathrm{~m}$ & meloads stoss:ten \\
\hline :urgeten imp & 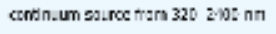 & melosus stex:ben \\
\hline Xe are in: & carrn.um gu.roc tran 210 100: nח & melowar fucrecen:s \\
\hline$=\boldsymbol{H}|>|$ a & 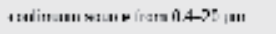 & "elenie atarclin \\
\hline 81000 & corrn.um ss.roc trans $110 . \mathrm{m}$ & melosus stexs:ben \\
\hline thathome mre & cerrn.um so.roc tran $03520 \mu \mathrm{m}$ & melogus stox:ben \\
\hline nelew ratheda tam & Ira sn.me in I.Q, Vkhs & aterv atserthen \\
\hline 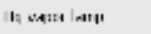 & 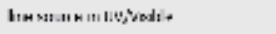 & 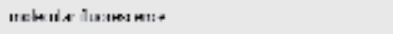 \\
\hline Inom & 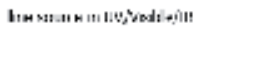 & 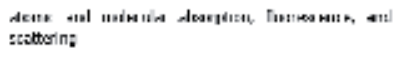 \\
\hline
\end{tabular}

Table 1.

The most common kinds of light sources.

provide a better performance for both AES and sample introduction into an inductively coupled plasma. Traditional sources usually include arcs and sparks but modern instruments use argon or some other inert gas to create plasma. The plasma may be produced when gas atoms are ionized, $\mathrm{Ar}+\mathrm{e}^{-} \rightarrow \mathrm{Ar}^{1}+2 \mathrm{e}^{-}-$a process generated by seeding ions from a high-voltage spark-and is sustained from a radio frequency generator in the area of the induction coil. This is known as inductively coupled plasma (ICP). Plasma exists at temperatures of up to $10,000 \mathrm{~K}$ and the instrument prevents the torch from melting. XRF requires that sample should be irradiated by high energy photons. In most instruments, the source is the polychromatic primary beam from $X$-Ray tubes. Of interest to biological applications, however, it is the use of radioactive isotopes such as ${ }^{244} \mathrm{Cm},{ }^{241} \mathrm{Am},{ }^{55} \mathrm{Fe}$, and ${ }^{109} \mathrm{Cd}[11,12]$.

An ideal wavelength selector has a high throughput of radiation and a narrow effective bandwidth. There are two major types of wavelength selectors - filters and monochromators. A simple example of an absorption filter is a piece of colored glass. Absorption filters provide effective bandwidths of $30-250 \mathrm{~nm}$, although the throughput can be only $10 \%$ of the source's emission intensity at the low end of this range. Interference filters constructed of a several optical layers deposited on a glass or transparent material. Typically, effective bandwidth is $10-20 \mathrm{~nm}$, with maximum throughputs of at least $40 \%$ [11]. A monochromator is used to convert a polychromatic source of radiation at the entrance slit to a monochromatic source of restricted effective bandwidth at the exit slit. These devices are classified as either fixed-wavelength or scanning. The wavelength selects by manually rotating the grating in a fixed-wavelength monochromator. A scanning monochromator includes a drive mechanism that continuously rotates the grating, allowing sequential wavelengths to exit from the monochromator (Figure 3) [11].

Detectors use a sensitive transducer that converts a signal comes from light energy into electrons An ideal detector produces signal, $S$, is a linear function of the electromagnetic radiation's power, $P$,

$$
\mathrm{S}=\mathrm{kP}+\mathrm{D}
$$

where $k$ is the detector's sensitivity and $D$ is the detector's dark current, or the background current when no radiation of source reached to the detector.

Phototubes and photomultipliers include a photosensitive surface that absorbs radiation in the $\boldsymbol{U V}$-visible, or near-IR, generating an electrical current proportional to the number of photons reaching the transducer (Figure 4). Other photon 
(a)
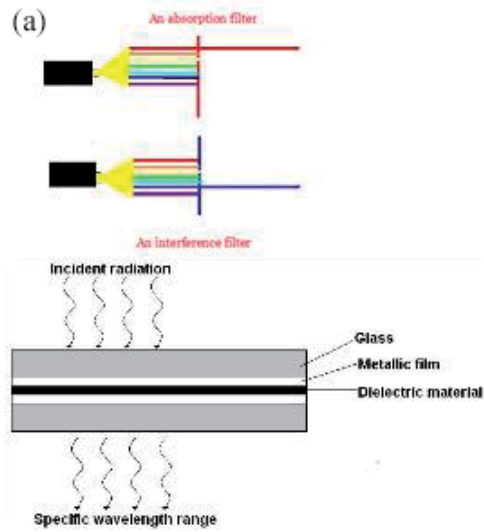

(b)

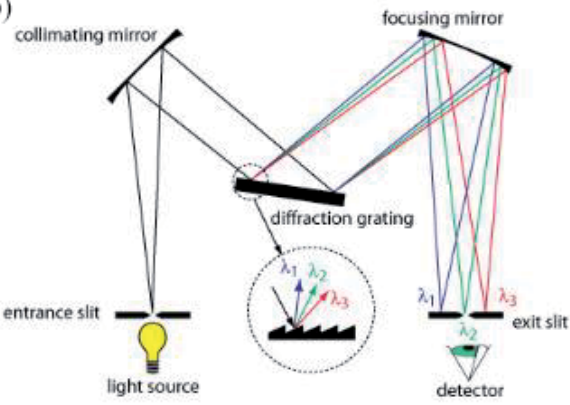

Figure 3.

Schematic diagram of wavelength selectors: (a) filters and (b) a diffraction grating monochromator.
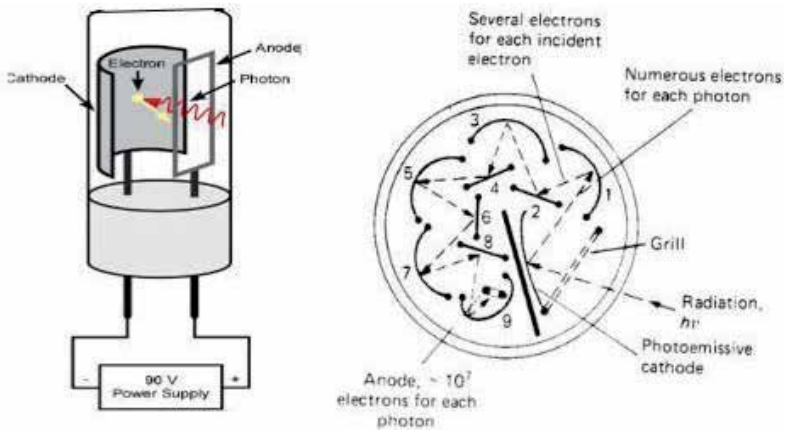

Figure 4 .

Diagram of a phototube and a photomultiplier tube.

detectors use a semiconductor compound as the photosensitive surface. One advantage of the Si photodiode manufactured utilizing semiconductor process is that it easy to miniaturize. Infrared photons do not have enough heat to generate a measurable current with a photon transducer [11].

A transducer's electrical signal is sent to a signal processor where it is displayed in a form that is more convenient to explain. The analog meters, digital meters, recorders, and computers equipped with data acquisition boards are good examples of signal processors. A signal processor is used in calibrating the detector's

\begin{tabular}{llcl}
\hline Detector & Class & Wavelength Range & Output Signal \\
\hline phototube & photon & $200-1000 \mathrm{~nm}$ & current \\
photomultiplier & photon & $110-1000 \mathrm{~nm}$ & current \\
Si photodiode & photon & $250-1100 \mathrm{~nm}$ & current \\
photoconductor & photon & $750-6000 \mathrm{~nm}$ & change in resistance \\
photovoltaic cell & photon & $400-5000 \mathrm{~nm}$ & current or voltage \\
thermocouple & thermal & $0.8-40 \mu \mathrm{m}$ & voltage \\
thermistor & thermal & $0.8-40 \mu \mathrm{m}$ & change in resistance \\
pneumatic & thermal & $0.8-1000 \mu \mathrm{m}$ & membrane displacement \\
pyroelectric & thermal & $0.3-1000 \mu \mathrm{m}$ & current
\end{tabular}

Table 2.

Examples of detectors for spectroscopy. 
response, amplificating the transducer's signal, removing noise by filtering, or mathematically transforming the signal [11] (Table 2).

\section{Atomic spectroscopy: sample preparation}

An ideal sample preparation should remove interfering components from the matrix and to adjust of analyte to facilitate the actual measurement. Methods for destruction of the organic matrix by simple heating or by acid digestion have been developed and are thoroughly approved. Microwave heating is used for this purpose, with the specifically designed a compatible equipment to avoid dangerous of excessive pressure within reaction flask. Although the number of samples that can be processed is not large, microwave heating affords rapid digestion and low reagent blanks. More recent developments include continuous flow systems for automated digestion which has a direct link with the instrument [12].

Liquid-liquid portioning has been widely applied for preconcentration procedure. Analyte atoms in a large volume of aqueous solution are complexed with a suitable agent and collected into a small volume of solvent. Vapor generation procedures permit the rapid introduction of $100 \%$ of the sample into the atomizer and are used for AAS, AES, AFS, and ICP-MS. Certain elements such as arsenic, selenium, and bismuth readily evolve gaseous hydrides and transferred by a flow of inert gas to an AES, and ICP-MS or to a heated silica tube positioned in the light path for AAS, AFS. The tube can be heated using the air-acetylene flame or an electric current. The obtained heat is enough to cause decomposition of the hydride and atomization of the analyte. Thus, there is no loss off analyte, which in all the atoms flow the light path with in few seconds and they are trapped within the silica tube that was retarded their dispersion. Any sample volume added to the reaction container, hydride generation AAS has detection limits a few nanograms of analyte. Mercury can quickly form a vapor in the ambient temperature, and this property is the basis for cold vapor generation. When a reducing agent is added to sample solution, $\mathrm{Hg}^{2+}$ converts to the elemental mercury. Agitation or bubbling of gas through the solution is used to enhance rapid vaporization of the atomic mercury and to improve the transfer of mercury to a flow through cell located in the light path. As with hydride generation, the detection limit is a few nanogram and some manufacturers have been developed common instrumentation to accomplish both procedures. Chromatographic or electrophoretic techniques have been also developed that are coupled directly to the atomic spectroscopic instrument to develop integrated analytical arrangements [13].

\section{Atomic spectroscopy: detection limits}

The detection limits are important parameters of analytical techniques. Typical detection limit ranges for the major atomic spectroscopy techniques are shown in Figure 5. AAS detection limits are generally better in all cases where the element can be atomized. Detection limits for refractory elements such as bor, titanium, and vanadium are better by ICP than by AAS. Nonmetals and the halogens can only be determined by ICP. Optimum detection of nonmetals such as sulfur, nitrogen, and halogens by ICP-ES can only be achieved when a vacuum monochromator is used. For mercury and those elements that form hydrides, the cold vapor mercury or hydride generation techniques offer exceptional detection limits [14]. 


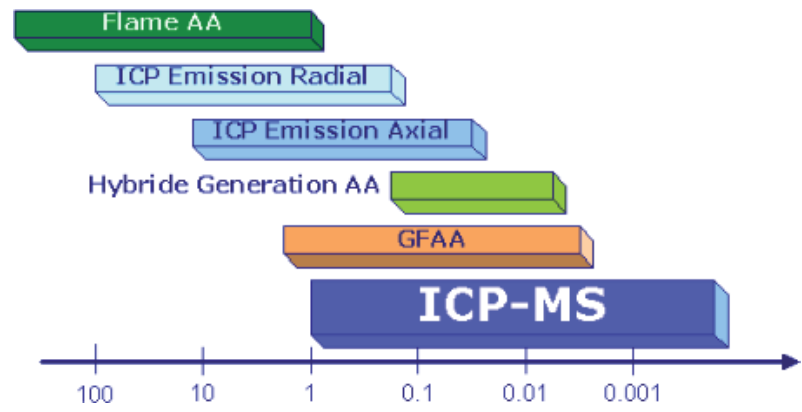

Figure 5 .

Typical detection limit ranges for the major atomic spectroscopy techniques.

\section{Atomic spectroscopy: analytical working range}

The analytical working range can be considered as the concentration range over which quantitative results can be obtained without recalibration for system. Selecting a technique with an analytical working range based on the expected analyte concentrations, minimizes the analysis times by allowing the samples with different analyte concentrations to be analyzed together. For example; ICP-MS, once considered only an ultratrace element technique, can now run concentration ranges from low parts-per-trillion (ppt) level up to high parts per million (ppm). A wide analytical working range also can reduce, for example handling requirements, minimizing potential errors. Figure 6 shows typical analytical working ranges with a single set of instrumental conditions [15].

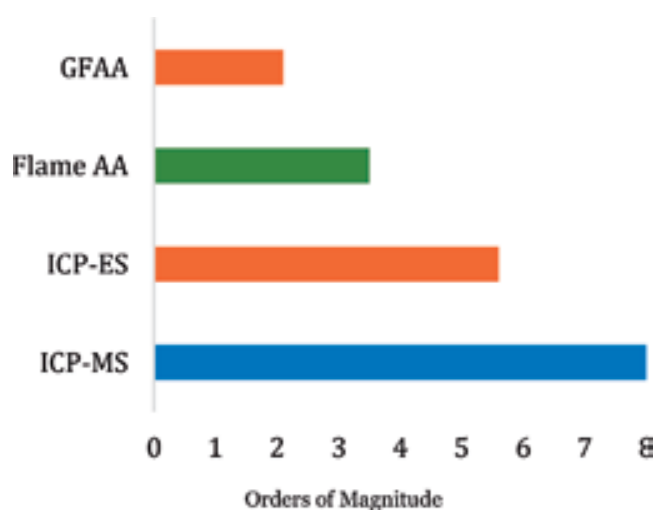

Figure 6.

Analytical working ranges for the major atomic spectroscopy techniques.

\section{Atomic spectroscopy: interferences}

Spectroscopic interferences have been determined and documented, and methods have been used to correct or compensate for those interferences which may occur. For example; ICP-AES provides a wide dynamic range and minimal chemical interferences [15]. A summary of the types of interferences seen with atomic spectroscopy techniques, and the corresponding methods of compensation are shown in Table 3. 


\begin{tabular}{lll}
\hline Technique & Type of interference & Method of compensation \\
\hline Flame AA & lonization & lonization buffer \\
& Chemical & Releasing agent or nitrous oxide-acetylene flame \\
& Physical & Dilution, matrix matching, or method of additions \\
\hline \multirow{2}{*}{ GFAA } & Physical and chemical & STPF conditions \\
& Molecular absorption & Zeeman or continuum source background correction \\
& Spectral & Zeeman background correction \\
\hline \multirow{2}{*}{ ICP emission } & Spectral & Background correction or the use of alternate \\
& Matrix & analytical lines \\
& & Internal standardization \\
\hline \multirow{2}{*}{ ICP-MS } & Mass overlap & Inter element correction, use of alternate mass \\
& Matrix & values, or higher mass resolution \\
& & Internal standardization \\
\hline
\end{tabular}

Table 3.

Atomic spectroscopy interferences.

\section{Atomic spectroscopy: other performance criteria}

Performance criteria for analytical techniques include the ease of use, required operator skills, and availability of documented methodology. Table 4 summarizes comparative advantages and limitations of the most common atomic spectroscopy techniques.

\begin{tabular}{llllll}
\hline Criteria & Flame AA & Flame AE & AFS & ICP & X-RF \\
\hline Costs & Low $(\sim 10-15 \mathrm{~K})$ & Moderate & Moderate & Highest $(\sim 200 \mathrm{~K})$ & Highest \\
\hline Instrumental ion & Low & Low & High & High & High \\
\hline Maintenance & Low & Low & Low & High & High \\
\hline Sample preparation & Moderate & Low & & Moderate & High \\
\hline Speed & Slow & Medium & & Fast & Fast \\
\hline Operator skill & Lower & Moderate & Higher & Higher & Higher \\
\hline
\end{tabular}

Table 4.

Comparison of spectroscopic techniques performance.

\section{Atomic spectroscopy: recent developments and applications}

Analytical methods of atomic spectroscopy have been used for elemental analysis identification, and quantitation in varieties of samples. Recently, most all of the spectroscopic techniques available are used in the analysis of metals and trace elements in samples of industrial and environmental origin.

Progress continues to develop in analytical spectroscopy as improvements are made to sensitivity, limits of detection, and availability. Recent development depends on instrumental adjustments and slight modifications to allow new types of measurements. Advancements in materials science have revealed demand for new methods of measurement using instruments already accessible, pushing the boundaries of what was previously available. For example, some new and interesting miniaturized plasma sources and a new distance of flight (DOF) mass spectrometer have been to the fore in developments. In addition, several novel methods have been developed, such as laser ablation molecular isotopic 
spectrometry (LAMIS) for isotope ratio analysis, and stand-off LIBS techniques such as "underwater LIBS" [16].

\section{Conclusion}

This chapter summarizes the key principles and application areas of atomic spectroscopy techniques. For example, a medical laboratory could determine the type and amount of heavy metals that could be present in patient's serum or urine. Environmental scientists could monitor heavy metal contamination of water and soil. The pharmaceutical industry uses these techniques to determine metals and metalloids in drug products $[17,18]$.

Important criteria for selecting an analytical technique include detection limits, analytical working range, sample preparation, cost, ease of use, and the availability of proven methodology. Atomic spectroscopy techniques have provided a rapid, simple, accurate, and highly sensitive means of determining the concentrations of the elements.

In the future, it seems more likely that maximum permissible limits for elements in drinking water, the drug product etc. will be reduced, rather than increased, therefore more sensitive techniques, such as ICP-MS, will begin to play a greater role in the analysis of elements.

\section{Author details}

Deniz Sahin

Department of Chemistry, Faculty of Sciences, Gazi University, Ankara, Turkey

*Address all correspondence to: dennoka1k@hotmail.com

\section{IntechOpen}

(C) 2019 The Author(s). Licensee IntechOpen. This chapter is distributed under the terms of the Creative Commons Attribution License (http://creativecommons.org/licenses/ by/3.0), which permits unrestricted use, distribution, and reproduction in any medium, provided the original work is properly cited. (cc) BY 


\section{References}

[1] Taylor A. Atomic spectroscopy, biomedical applications. In: Lindon CJ, Tranter EG, Koppanaal WD, editors. Encyclopedia of Spectroscopy and Spectrometry. 3rd edn. Netherland: Elsevier Ltd; 2017. pp. 76-80

[2] Nicholas PC. Elemental and structural characterization tests. In: Nicholas PC, editor. Polymer Characterization, Laboratory Techniques and Analysis. New Jersey, USA: Noyes Publications; 1996. pp. $43-81$

[3] Mahmoud N, Monireh A, Mohaddeseh S, Mohammad SS, Zahra I. Interface science and technology. Chapter 6. In: Nasrollahzadeh M, Mohammad SS, Sajjadi M, Zahra I, Monireh A, editors. Plant-Mediated Green Synthesis of Nanostructures: Mechanisms, Characterization, and Applications. Vol. 28. Netherland: Elsevier Ltd.; 2019. pp. 199-322

[4] John AT, Vaughan DJ. Theoretical Geochemistry: Applications of Quantum Mechanics in the Earth and the Mineral Sciences. UK: Oxford University Press; 1992. p. 20

[5] Wincott PL, Vaughan DJ. Sulfide Mineralogy and Geochemistry. Chapter 4. In: Vaughan DJ, editor. Spectroscopic Studies of Sulfides. Vol. 61. USA: Mineralogical Society of America; 2006. p. 191

[6] Andrew T. Biomedical applications of atomic spectroscopy. In: Lindon CJ, editor. Encyclopedia of Spectroscopy and Spectrometry. UK: Academic Press; 1999. pp. 174-182

[7] Flanagan Robert J, Taylor Andrew A, Ian DW, Robin W. Fundamentals of Analytical Toxicology. WileyInterscience: Wiley-Interscience; 2008. pp. 281-317

[8] Brouwers EE, Tibben M, Rosing H, Schellens JH, Beijnen JH.
The application of inductively coupled plasma mass spectrometry in clinical pharmacological oncology research. Mass Spectrometry Reviews.

2008;27:67-100

[9] Ramyalakshmi G, Venkatesh P, Hepcy KD, Ravindra Reddy K, Archana E, Manjuvani S. A review on inductively coupled plasma mass spectroscopy. International Journal of Drug Development and Research. 2012;4:69-79

[10] Harvey D. Spectroscopic methods. Chapter 10 In: Harvey D, editor.

Analytical Chemistry 2.0. USA: The California State University Affordable Learning Solutions Program, and Merlot; 2019. pp. 552-603

[11] Overview of Spectroscopy. Available from: https://chem.libretexts.org/ Courses/Northeastern-University/ Spectroscopic\%20Methods/10.1

[12] Farrukh MA, editor. Atomic Absorption Spectroscopy. USA: InTech; 2011

[13] Andrew T. Biomedical photonics handbook. In: Vo-Dinh T, editor. Atomic Spectroscopy in Biological and Clinical Analysis. Vol. 23(1). USA: CRC Press; 2003. p. 11

[14] Chunlong Z, editor. Fundamentals of Environmental Sampling and Analysis, Chapter 9. 1st ed. Hoboken, New Jersey, USA: John Wiley \& Sons, Inc.; 2000. p. 236

[15] The guide to techniques and applications of atomic spectroscopy. An Overview of Atomic Spectroscopy, Section 1. Waltham, USA: Perkin Elmer, Inc.; 1997. pp. 2-47

[16] Evans EH et al. Atomic spectrometry updates: Review of advances in atomic spectrometry 
and related techniques. Journal of Analytical Atomic Spectrometry. 2014;29:773-794

[17] Walsh A. The application of atomic absorption spectra to chemical analysis. Spectrochimica Acta. 1955;7:108-117

[18] Alkemade CTJ, Milatz JMW. Double-beam method of spectral selection with flames. Journal of the Optical Society of America A. 1955;45:83-84 



\title{
What Is Vibrational Raman Spectroscopy: A Vibrational or an Electronic Spectroscopic Technique or Both?
}

\author{
Søren Hassing
}

\begin{abstract}
Modern Raman spectroscopy covers several noninvasive reflection techniques for identification of molecules and investigation of molecular properties. All are based on the Raman effect, occurring when polarized laser light is inelastically scattered by a molecular sample. Vibrational Raman spectroscopy is the Raman technique most widely used in chemical analysis, and it is relevant for the characterization of molecules in solution, biomolecules, and solids (crystals and powders). In this chapter vibrational Raman spectroscopy is discussed under the headline: "What is vibrational Raman spectroscopy: a vibrational or an electronic spectroscopic technique, or both?" The answer to this question is gained through a discussion of different scattering situations, and it is demonstrated that the Raman technique is more than an alternative technique to infrared and near-infrared absorption spectroscopy. Starting with the Kramers-Heisenberg equation, the answer is obtained through a discussion of: State tensors and Raman tensors, nonresonance and resonance Raman scattering (RRS) excited with a single laser wavelength, and unpolarized and polarization-resolved RRS excited with variable laser wavelengths (RADIS), dispersive Raman vibrations as a tool for noninvasive detection of molecular color changes and that RADIS data are automatically born as three-dimensional multivariate data with high information contents.
\end{abstract}

Keywords: Raman, resonance Raman, RADIS, polarization dispersion, state tensor, Raman tensor, noninvasive

\section{Introduction}

Modern Raman spectroscopy is a class of well-documented, noninvasive, optical reflection techniques with a high spectral resolution applicable for the identification of molecules and investigation of molecular properties. All are based on the Raman effect, discovered by Raman in 1928 [1, 2]. Today more than 25 different Raman spectroscopic techniques are known [3]. The Raman effect occurs when light is inelastically scattered by a molecular sample.

Originally Raman and Krishnan observed the scattering of spectrally filtered sunlight from a liquid and found that the scattered light contained very week 
components of light, which had slightly different frequencies compared to the frequency of the incoming light. In a description of the scattering process based on quantum mechanics, the appearance of the shifted frequencies in the Raman scattered light is interpreted in the way that the molecules have shifted quantum state during the process. The shifted frequencies appear symmetrically around the frequency of the exciting light, from which one can conclude that the molecule may either be excited or de-excited during the scattering process. The last requires that the molecules have been excited (e.g., thermally) before the scattering event. This is termed anti-Stokes scattering, while the scattering where the molecules are excited during the process is termed Stokes scattering. The molecules may either shift rotational, vibrational, or electronic state during the scattering, depending on the molecule and the specific experimental conditions.

The spectroscopic technique based on Raman scattering, where the molecules shift vibrational state, is termed vibrational Raman spectroscopy. A vibrational Raman spectrum contains the unique and highly resolved vibrational signature of the scattering molecule. Normally only the Stokes part of the entire spectrum is measured, since this is more intense than the anti-Stokes part [3]. Vibrational Raman spectroscopy is the Raman technique most widely used in chemical analysis, and it is relevant for the investigation of molecules in solution, biomolecules, and solids (crystals and powders). Since the Raman technique can be performed as a reflection measurement, which requires no or very little sample preparation, it is well suited for the investigation of molecules in their natural environment such as in the food industry and in medical and environmental applications.

Nowadays vibrational Raman spectra are measured by illuminating the sample with polarized laser light with wave numbers either in the near-infrared (NIR), the visible (VIS), or the ultraviolet (UV) and simultaneously monitoring the reflected light. A vibrational Raman spectrum is then obtained by considering the intensity distribution in the Raman scattered light as a function of the so-called Raman shift $\Delta \widetilde{\nu}_{R}$ defined as $\Delta \widetilde{\nu}_{R}=\widetilde{\nu}_{\text {laser }}-\widetilde{\nu}_{s}$, where $\widetilde{\nu}_{s}$ and $\widetilde{\nu}_{\text {laser }}$ are the wave numbers of the Raman scattered light and the laser, respectively. In the case of Stokes scattering, $\Delta \widetilde{\nu}_{R}$ is positive.

Ever since the discovery of the Raman effect in 1928, the Achilles heel of Raman spectroscopy has been the low Raman cross section where typically $10^{8}$ incoming photons only generate a single Raman photon. The consequence is that the intensity of the Raman signal becomes very low in general. In the history of Raman spectroscopy, many attempts have been made to overcome this disadvantage. The three most important milestones for the practical application of Raman spectroscopy are the following:

- The invention and development of commercial lasers $[4,5]$

- The development of sensitive charge-coupled devices (CCD-detectors) $[6,7]$

- The development of interference filters, i.e., notch and edge filters

Not before the implementation of these three improvements could Raman spectroscopy really compete with the competing spectroscopic techniques IR, NIR, and UV/VIS.

A challenge particular in in situ resonance Raman investigations of biomolecules is that the excitation of the resonance Raman process is followed by a simultaneous excitation of fluorescence in either the molecule under investigation or in other molecules present in the sample. Since in general the fluorescence cross section is 
much larger than the Raman cross section, the Raman peaks typically ride on top of the spectrally broad fluorescence background so that it can be time-consuming to determine the true Raman intensity. Sometimes a part of the Raman spectrum may even be hidden behind the fluorescence. However, in most situations the influence from fluorescence may be taken care of by changing the laser wave number to NIR or to UV, by applying a fluorescence quenching technique [8], by applying a pulsed detection technique, or by implementing advanced signal processing including multivariate analysis.

In the present chapter vibrational Raman spectroscopy is discussed under the headline: "What is vibrational Raman spectroscopy: a vibrational or an electronic spectroscopic technique, or both?" Besides giving an answer to this question, the goal of the chapter is twofold: (1) to improve the readers' understanding of Raman scattering in general and (2) to demonstrate which kind of molecular information one may achieve by choosing different experimental conditions.

Before we begin the discussion, it should be noticed that Raman spectroscopy, like any kind of molecular spectroscopy, can be applied in two different ways: (1) as an analytical technique for the identification and quantification of molecules in a sample and (2) as a technique for studying the physical and chemical properties of molecules. In the first kind of application, the Raman signal is just considered as a source of data, which has to be compared with spectroscopic databases or which has to be combined with the chemometric method being most appropriate for the problem to be solved. For this kind of application, no knowledge of Raman theory and how the theory is applied to molecules is really needed. But it should be noticed that to decide how the chemometrics should be applied together with the Raman data, one must take into account that vibrational Raman spectra are in general characterized by exhibiting high spectral resolution (narrow and well-separated lines) compared to the broader bands typically found in IR and in particular in UV/ VIS spectra. For the second kind of applications, a deeper understanding of molecular physics and molecular Raman theory is needed.

\section{Some fundamentals of Raman theory}

\subsection{General theory}

A unified treatment of Raman theory can be found in $[9,10]$ and in [3], where in the last reference a long list of references to the Raman literature is provided. The symmetry aspects, interference phenomena, and polarization properties of resonance Raman scattering have been discussed by Mortensen and Hassing [11] and by Schweitzer-Stenner [12], while the vibronic aspects has been discussed by Siebrand and Zgierski [13].

Raman scattering can be described as a coherent absorption-emission sequence in which a primary photon with wave number $\widetilde{\nu}_{p}$ and polarization vector $u_{p}$ is replaced by a scattered photon with wave number $\widetilde{\nu}_{s}$ and polarization vector $u_{s}$. In comparison, fluorescence is an incoherent absorption-emission sequence, i.e., a combination of two independent processes, namely, a real absorption of a primary photon followed by a spontaneous emission of a secondary photon. In fluorescence the initially excited molecule is allowed to decay into other quantum states before the spontaneous emission of light. As well known, the number of vibrations in a molecule is given by the expression $3 N-6$, where $N$ is the number of atoms in the molecule. Since each vibration can be highly excited, it follows that the total number of vibrational states associated with every electronic state including the 
electronic ground state increases strongly with the size of the molecule. This gives many possibilities for the decay and accordingly also for the emission of the light. When the intensity contributions from all the possible radiative transitions are superposed to give the total intensity, the individual spectra overlap with the result that the spectral distribution of the fluorescence becomes broad and without much structure. In contrast, the number of accessible final states in the Raman process is very limited due to the coherent nature of the process. The typical vibrational Raman spectrum consists of narrow Raman lines with the typical FWHM bandwidths of $10-20 \mathrm{~cm}^{-1}$.

The basic equation for the theoretical description of Raman scattering is the famous Kramers-Heisenberg $(\mathrm{KH})$ dispersion relation. Kramers and Heisenberg derived the equation by the application of the correspondence principle to the classical dispersion relation. The $\mathrm{KH}$ equation expresses the transition probability per second for Raman scattering [14]. The original version did not contain the damping of the scattering system, and thus it did not immediately apply to resonance Raman scattering. Later Weisskopf modified the equation by introducing the damping of the atomic and molecular states with the assumption of an exponential decay of the excited states [15]. Within a modern theoretical framework, the KH relation and the expression for the intensity of the Raman scattered light can be derived by using formal scattering theory [16] or time-dependent second-order perturbation theory. In perturbation theory the interaction energy between the molecule and radiation field, normally considered in the electric dipole approximation, is used as the perturbation, when the time-dependent Schrödinger equation for the total system and molecule plus electromagnetic field is solved (e.g., see [17]).

The basic scattering equations are collected in Eq. (1). The transition probability is expressed through the total differential scattering cross section $\left(\frac{d \sigma}{d \Omega}\right)=\frac{I_{\text {Raman }}}{I_{\text {laser }}}$. Since the intensity of the laser $I_{\text {laser }}$ refers to a plane wave and the intensity of the Raman scattered light $I_{\text {Raman }}$ is the intensity scattered into the solid angle $d \Omega$, the scattering cross section $\left(\frac{d \sigma}{d \Omega}\right)$ becomes an area. It follows from Eq. (1) that the intensity of the Raman scattered light corresponding to the Raman transition $|a\rangle \rightarrow|b\rangle$ is proportional to the intensity of the laser $I_{\text {laser }}$ and to the absolute square of the Raman tensor, $\left|\alpha^{a \rightarrow b}\right|^{2}$, where $\alpha^{a \rightarrow b}$ is a tensor of rank 2 represented by a $3 \times 3$ matrix, which is related to the electric polarizability of the molecule. The expression for the Cartesian components of the Raman tensor $\alpha_{\rho \sigma}^{a \rightarrow b}(\rho \sigma=x, y, z)$ is also given in Eq. (1):

$$
\begin{gathered}
I_{\text {Raman }}=\left(\frac{d \sigma}{d \Omega}\right) I_{\text {laser }} d \Omega \\
\left(\frac{d \sigma}{d \Omega}\right)=4 \pi \alpha_{f s c}^{2} \widetilde{\nu}_{s}^{4}\left|\sum_{\rho \sigma} u_{s \rho} \alpha_{\rho \sigma}^{a \rightarrow b} u_{p \sigma}\right|^{2} \\
\alpha_{\rho \sigma}^{a \rightarrow b}=\sum_{r} \frac{\langle b|\rho| r\rangle\langle r|\sigma| a\rangle}{\widetilde{\nu}_{r, a}-\widetilde{\nu}_{p}-i \gamma_{r}}+\frac{\langle b|\sigma| r\rangle\langle r|\rho| a\rangle}{\widetilde{\nu}_{r, b}+\widetilde{\nu}_{p}+i \gamma_{r}}
\end{gathered}
$$

$\alpha_{f s c}$ is the fine structure constant known from atomic physics [17]. Notice that the Raman intensity is proportional to the fourth power of the wave number of the scattered light (i.e., inversely proportional to the fourth power of the wavelength). This means that the intensity of a Raman spectrum measured with a NIR laser with wavelength $1064 \mathrm{~nm}$ is decreased with a factor of 16 relative to the intensity of the same spectrum measured with a visible laser with wavelength $532 \mathrm{~nm}$. 
In the expression for the Raman tensor in Eq. (1), $|a\rangle,|b\rangle$ and $|r\rangle$ is the initial, the final, and the intermediate states of the molecule. In principle, all states are exact eigenstates of the molecular Hamiltonian. $\rho, \sigma$ is a shorthand notation for the Cartesian components of the electric dipole moment of the molecule divided by the velocity of light and measured relative to a coordinate system in the center of mass of the molecule. $\widetilde{\nu}_{r, a}$ is the wave number difference between the states $|r\rangle$ and $|a\rangle$. $\gamma_{r}$ is the damping of the state $|r\rangle$ given as the FWHM width and represents the exponential decay of the state. The matrix elements in the numerators of the two terms in the Raman tensor are given in the Dirac notation [18] and involve mathematically an integration over the coordinates used in the functions describing the molecular states. The terms in the expression for the Raman tensor, where the denominators contain the wave number difference between the state $|r\rangle$ and the laser $\widetilde{\nu}_{p}$, are called the resonance terms, since their contributions to the Raman intensity increase strongly when the excitation wave number $\widetilde{\nu}_{p}$ becomes equal to their energies $\widetilde{\nu}_{r, a}$. When this happens the scattering process is called resonance Raman scattering (RRS), while in cases where $\widetilde{\nu}_{p} \ll \widetilde{\nu}_{r, a}$, the process is just called Raman scattering (RS). The difference between RRS and RS and the implications with respect to the kind of molecular information one may obtain in the two scattering situations will be discussed in more detail in the following.

It follows from Eq. (1) that all the molecular states contribute to the intensity of the specific Raman transition $|a\rangle \rightarrow|b\rangle$. It also follows that the first step in the calculation of the intensity is to calculate the Raman tensor by performing the summation over all molecular states and then as the second step calculate the absolute square of the result. The consequence is that the contribution from each state will interfere either constructively or destructively with the contributions from all other states in the Raman intensity. The interference between the individual contributions depends on their magnitude and relative sign. An important issue in the evaluation of the expression for the Raman intensity is to establish the relations between the molecular properties and the Raman process. To achieve this goal, it is appropriate to divide the discussion into two parts: (1) discussion of the contribution to the scattering tensor from a single state and (2) discussion of the interference between the contributions from different states. For that purpose Mortensen and Hassing [11] defined the state tensor $S_{\rho \sigma}^{|r\rangle}$ corresponding to the intermediate state $|r\rangle$ :

$$
S_{\rho \sigma}^{|r\rangle} \equiv\langle b|\rho| r\rangle\langle r|\sigma| a\rangle
$$

The state tensor determines the contribution to the scattering from that particular state. $S_{\rho \sigma}^{|r\rangle}$ depends also on the initial and final states of the Raman process $|a\rangle$ and $|b\rangle$, but we omit these indices for simplicity in writing.

The Raman tensor now becomes

$$
\alpha_{\rho \sigma}^{a \rightarrow b}=\sum_{r} \frac{S_{\rho \sigma}^{|\gamma\rangle}}{\widetilde{\nu}_{r, a}-\widetilde{\nu}_{p}-i \gamma_{r}}+\frac{S_{\sigma \rho}^{|r\rangle}}{\widetilde{\nu}_{r, b}+\widetilde{\nu}_{p}+i \gamma_{r}}
$$

The introduction of the state tensor is more important in RRS, where only relative few states contribute significantly to the Raman intensity than in RS, where all molecular states contribute with the result that the information of their individual contributions is blurred.

One advantage of introducing the state tensor is that it is possible to evaluate the general form of the state tensor in cases, where the symmetry of the molecule is 
known. First, we notice that when the molecule has no symmetry, the state tensors are determined exclusively by the physical properties of the specific molecule considered, and all state tensor components may a priori be nonvanishing. The same holds for the Raman tensor, since it is a superposition of state tensors scaled with the associated energy factors. However, when the molecule exhibits some symmetry, the state tensors will be determined both by the physics and by the constraints imposed by the symmetry. The consequence of the symmetry is that some tensor components vanish. When the symmetry gets higher, the number of vanishing state tensor components increases. It is also important to notice that the structure of each state tensor contributing to a specific Raman transition can be different and different from the structure of the Raman tensor. This is because the state tensors are determined by the symmetry and the physical properties of the individual states, which contribute to the scattering, while the Raman tenor is a superposition of the state tensors. As we shall see later, this is particularly important in resonance Raman scattering of molecules containing a chromophore with high symmetry such as the molecules containing the heme group.

Traditionally the symmetry of a molecule with well-defined configuration has been described by using point groups and group representation theory, and from the early days of quantum physics, this has been used to derive selection rules, i.e., to give the conditions under which a particular matrix element must vanish. In spectroscopy the symmetry-based selection rules determine when a transition does not appear in the spectrum, i.e., it is a symmetry-forbidden transition.

In cases where the molecular point group has a threefold rotation axis or axes of higher order, one must, however, as demonstrated in [11] and by Mortensen in [19], apply the so-called non-commuting generator (NCG) approach to molecular symmetry in order to be able to evaluate the general form of the state tensors. In [19] the NCG approach is explained in detail, while in [11] it is shown how the method can be applied to calculate the structure of the state tensors both for molecules with integer and half integer spin. Besides all the possible state tensors for molecules with integer spin in the most important point groups have been evaluated and collected as an appendix. The appendix containing the state tensors is also reproduced in [3].

Recently [20] the NCG method has been extended and applied to develop the state and Raman tensors for molecular aggregates. Specifically the tensors for the $\mathrm{H}$ type dimer of two coupled monomers with $D_{4 h}$ symmetry are evaluated, and the result has been applied to interpret the experimental results of polarized resolved RRS spectra of a diluted solution of red blood cells (RBCs) of human blood. The main conclusion from this study is that aggregation between heme-protein molecules inside the RBC can be studied in vivo by polarized RRS, which opens the possibility of monitoring the effects on the aggregation of drugs added to the blood.

\subsection{Polarization properties of Raman scattering}

The polarization is a unique property of Raman scattering, which distinguishes the Raman technique from the UV/VIS and IR spectroscopy. In general, the polarization of the Raman scattered light is different from the polarization of the incoming laser light. This property is valid for oriented molecules (crystals), but perhaps more surprisingly, it is also valid for randomly oriented molecules like molecules in solutions and in powders. The reason for this is that the Raman process is controlled by a tensor (the Raman tensor) and not by a vector (the electric dipole vector) like in UV/VIS and IR absorption. While a vector only has one quantity, namely, its length, which is not changed (invariant) under rotation of the molecules, a tensor of rank 2 has three combinations of the nine tensor components, which are invariant 
to rotation. In the Raman case, these are called the rotational invariants of the Raman tensor, and they are denoted as $\Sigma^{0}, \Sigma^{1}$, and $\Sigma^{2}$. Recently the polarization properties of resonance Raman have been discussed in [20]. It is demonstrated that the amount of molecular information, which may be extracted from a resonance Raman experiment, can be increased considerably by measuring the polarizationresolved Raman signals in addition to the unpolarized signals. Specifically the results of two case studies are discussed. As already mentioned the first focuses on the aggregation of hemoglobin inside RBCs of human blood, and the second is an in vitro study of the stability of dye-sensitized solar cells. Here we shall only define the polarization properties relevant for the discussion that follows below, and we refer to $[11,20]$ for further details.

In polarization-resolved Raman experiments, two spectra are measured from which the parallel $\left(\frac{d \sigma}{d \Omega}\right)_{\|}$and perpendicular $\left(\frac{d \sigma}{d \Omega}\right)_{\perp}$ scattering cross sections can be determined by using the relation in Eq. (1).

The polarization properties in Raman scattering are expressed through the depolarization ratio (DPR) defined as

$$
D P R \equiv \frac{\left(\frac{d \sigma}{d \Omega}\right)_{\perp}}{\left(\frac{d \sigma}{d \Omega}\right)_{\|}}
$$

The polarization-resolved Raman measurements are illustrated in Figure 1, which shows the $180^{\circ}$ scattering geometry (reflection measurement) being the geometry mostly used. The molecule (M) is placed in the center of a space-fixed coordinate system, and the laser light, which is linearly polarized along the $Z$-axis, is propagating along the $X-$ axis in the negative direction. The Raman signals back reflected from the molecule are measured with a detector placed on the $X$-axis and with the polarization analyzers directed along the $Z$ - axis for measurement of the parallel component $\left|\alpha_{Z Z}^{a \rightarrow b}\right|^{2}$ (marked red in Figure 1) and along the $Y$-axis for

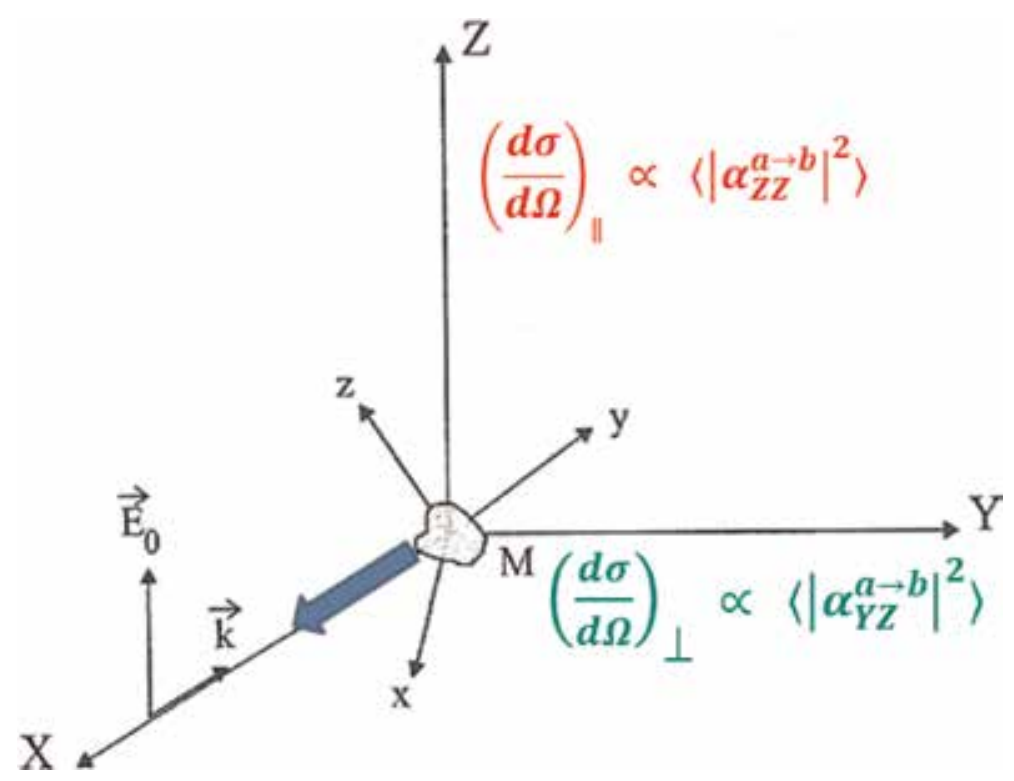

Figure 1.

Backscattering geometry illustrating polarized resolved Raman measurements of the parallel (along Z) and perpendicular (along Y) polarized data. $\overrightarrow{E_{0}}$ and $\vec{k}$ are the polarization and k-vector of the laser. $M$ is the randomly oriented molecule. 
measurement of the perpendicular polarized component $\left|\alpha_{Y Z}^{a \rightarrow b}\right|^{2}$ (marked green in Figure 1). For randomly oriented molecules, i.e., solutions and powders, we have to perform an average calculation of these scattering quantities with respect to orientation of the molecule. The average quantities $\left\langle\left|\alpha_{Z Z}^{a \rightarrow b}\right|^{2}\right\rangle$ and $\left\langle\left|\alpha_{Y Z}^{a \rightarrow b}\right|^{2}\right\rangle$ have been calculated in [11] by the application of angular momentum theory, and we refer to [11] for details. The result for the DPR is as follows:

$$
D P R=\frac{\left\langle\left|\alpha_{Y Z}^{a \rightarrow b}\right|^{2}\right\rangle}{\left\langle\left|\alpha_{Z Z}^{a \rightarrow b}\right|^{2}\right\rangle}=\frac{5 \Sigma^{1}+3 \Sigma^{2}}{10 \Sigma^{0}+4 \Sigma^{2}}
$$

where $\Sigma^{0}, \Sigma^{1}$, and $\Sigma^{2}$ are the rotational invariants mentioned above and each of them is a combination of the absolute squares of the Raman tensor components given in molecule-fixed coordinates [11]. $\Sigma^{0}$ is the absolute square of the trace of the tensor, $\Sigma^{2}$ is the symmetric anisotropy, and $\Sigma^{1}$ refers to the antisymmetric part of the Raman tensor, i.e., it contains terms of the form $\left|\alpha_{\rho \sigma}^{a \rightarrow b}-\alpha_{\sigma \rho}^{a \rightarrow b}\right|^{2}$.

\subsection{Vibronic expansion of the state and Raman tensors}

From the beginning of the history of Raman spectroscopy, it has been an important task to transform the basic and general scattering expressions into a form suitable for the interpretation of experimental Raman data. It follows from Eq. (1) that an important problem is that the calculation of the Raman intensity involves a summation over all molecular states, which are not known. Another problem is that in a molecule, the motions of the electrons and nuclei are not independent, which means that the functions describing the molecular states depend upon coordinates defining the positions of both the electrons and nuclei. It follows that any evaluation of the expression for the Raman intensity requires the introduction of approximations. It seems that there exist two different kinds of approach: the first approach introduces a number of radical approximations in one step, which leads to simple results, but with limited applicability. This approach was first followed by Placzek in 1934 with the development of his polarizability theory, which is valid for nonresonance Raman scattering [9]. The polarizability theory has been reconsidered by Long in 2002 [3] and is also briefly discussed below. The second approach introduces a number of approximations in a stepwise way directed toward Raman scattering in specific molecular systems. This kind of approach became relevant after the invention of the laser and development of commercial lasers, in particular tunable lasers in the 1960s and 1970s by which it became possible to perform resonance Raman experiments where the wave number of the laser was scanned through the visible absorption band of a large molecule. In particular, resonance Raman studies of biological molecules containing a chromophore came into focus [21-24]. Accordingly, it became necessary to transform the basic equations into a form suitable for the interpretation and extraction of the molecular information obtained under resonance conditions.

Almost all theoretical treatments of optical spectroscopic processes in molecules, including UV/VIS and Raman spectroscopy, are formulated within the adiabatic formalism. This formalism is based upon the assumption that in a molecule the motions of the light electrons are only weakly correlated to the motions of the heavy nuclei, so that it is possible to approximately separate these motions, when the Schrödinger equation for the molecule is solved. In this chapter we focus on 
vibrational Raman spectroscopy of larger molecules, i.e., either crystals or solutions and powders. In these molecules, the rotational spectra are not resolved, which means that it is not necessary to consider the rotational motion explicitly. In the adiabatic Born-Oppenheimer (ABO) approximation (e.g., see [17]), each molecular eigenstate is therefore, as a first step, approximated by a product of an electronic state and a vibrational state, where the ladder is normally considered within the harmonic approximation. Within the $\mathrm{ABO}$ approximation, the states in the state and Raman tensors are replaced by the product states: $|a\rangle=|g\rangle\left|v_{a}\right\rangle,|b\rangle=|g\rangle\left|v_{b}\right\rangle$ and $|r\rangle=|e\rangle\left|v_{e}\right\rangle$ where $g$ and $e$ are the electronic ground state and an excited electronic state, respectively. $\left|v_{e}\right\rangle=\left|v_{1}, v_{2}, v_{3}, v_{4}, \ldots \ldots v_{3 N-6}\right\rangle$ is the vibrational sub-state associated with the electronic state $e\rangle$, where in the harmonic approximation the vibrational state is also factorized in the product of $3 N-6$, and one-dimensional harmonic oscillator states, each of which describing a normal vibration. $N$ is the number of atoms in the molecule. However there are situations where the ABO approximation breaks down the product functions that serve in general as good approximations to the molecular wavefunctions. The most important situation for resonance Raman scattering, where it is necessary to go beyond the ABO approximation, is the one where an excited electronic state is coupled to the high-lying vibrational states belonging to the electronic ground state. The nonadiabatic coupling is mainly provided by the $\hat{T}_{N}$-operator, which is the operator describing the kinetic energy of the nuclei. This gives rise to radiationless transitions, which leads to an increase of the damping constant of the excited electronic state and explains essentially why the magnitude of the bandwidths observed in experimental UV/VIS absorption spectra is larger than the radiation damping. For the discussion given here, it is sufficient to associate the bandwidths estimated in UV/VIS spectra with the constant $\gamma_{r=e v}$ used in the Raman tensor. See $[25,26]$ for further discussions of the origin of the bandwidths found in Raman and UV/VIS experiments.

Since the ABO approximation only allows a partial separation of the electronic and nuclear motions, the functions describing the electronic states will depend on both the electronic coordinates and on the nuclear coordinates, while the vibrational functions only depend on the nuclear coordinates. However, the vibrational motion depends indirectly on the electronic state, since the electronic eigenvalue is a function of the nuclear coordinates and is found to play the role of the potential energy function for the vibrational motion.

The vibronic version of the state tensor in Eq. (2) may now be written as

$$
S_{\rho \sigma}^{|e\rangle\left|v_{e}\right\rangle}=\left\langle g(q, Q) v_{b}(Q)|\rho(q)| e(q, Q) v_{e}(Q)\right\rangle\left\langle e(q, Q) v_{e}(Q)|\sigma(q)| g(q, Q) v_{a}(Q)\right\rangle
$$

where $q$ and $Q$ symbolize the set of electronic and nuclear position coordinates, respectively. Thus each of the two matrix elements in the state tensor is evaluated by integrating formally over both the electronic and the nuclear coordinates. Besides, according to the adiabatic idea, the integration over the electronic coordinates should be performed before the integration over the nuclear coordinates.

In general, the functions describing the excited electronic states in larger molecules are not known. In fact, one important task in optical spectroscopy is to provide knowledge of these functions. Since also their dependence on the nuclear coordinates is not known, the formal integrations over the nuclear coordinates in the state tensor given in Eq. (6) are performed by introducing an appropriate Taylor expansion in the nuclear coordinates. The expansion of the state tensors may be performed in two different ways: (1) the electronic functions are expanded using perturbation theory and then the integration over the nuclear coordinates are performed (e.g., see [27]), and (2) the electronic transition moments $\rho(Q)=\langle g(q, Q)|\rho(q)| e(q, Q)\rangle$ and $\sigma_{e g}(Q)=\langle e(q, Q)|\sigma(q)| g(q, Q)\rangle$ defined through 
the formal integration over the electronic coordinates are Taylor expanded in the nuclear coordinates (e.g., see [11]). After the expansion of the state tensors in Eq. (6), using either scheme 1 or 2, the result is inserted in Eq. (1) in order to obtain the Raman intensity. Applying (2) to the first order (being in most cases sufficient), the state tensor in Eq. (6) becomes

$$
\begin{aligned}
S_{\rho \sigma}^{\left.|e| v_{e}\right\rangle}= & \left\langle v_{b} \mid v_{e}\right\rangle\left\langle v_{e} \mid v_{a}\right\rangle \rho_{g e}^{0} \sigma_{e g}^{0}+\sum_{k=1}^{3 N-6}\left\langle v_{b}\left|Q_{k}\right| v_{e}\right\rangle\left\langle v_{e} \mid v_{a}\right\rangle\left(\frac{\partial \rho_{g e}}{\partial Q_{k}}\right)_{0} \sigma_{e g}^{0} \\
& +\left\langle v_{b} \mid v_{e}\right\rangle\left\langle v_{e}\left|Q_{k}\right| v_{a}\right\rangle \rho_{g e}^{0}\left(\frac{\partial \sigma_{e g}}{\partial Q_{k}}\right)_{0}
\end{aligned}
$$

where all vibrational states are functions of the set of normal coordinate $Q$. The first and second lines are the state tensor in the Franck-Condon and the Herzberg-Teller approximations, respectively. Comparing Eq. (7) with the result in [27], it follows that the second expansion scheme leads to the simplest parametrization of the expression for the Raman intensity; although taken to infinite order, which is of course impossible in practice, the two expansion schemes are equivalent.

The vibronic models developed for RRS, which are found in the Raman literature, differ essentially from each other in two ways: (1) whether expansion scheme 1 or 2 has been applied and (2) the degree of approximation that has been introduced.

\section{What is vibrational Raman spectroscopy: a vibrational or an electronic spectroscopic technique or both?}

\subsection{Non-resonance Raman spectroscopy}

In most molecules, the energy differences between the electronic ground state and the excited electronic states are much larger that the mutual energy difference between the excited electronic states. Typically, there will therefore exist a relatively large spectral region between the energy of the final state in the Raman process and the energy of the first electronically excited state, where the wave number of the laser $\widetilde{\nu}_{p}$ can be chosen without exciting an electronic absorption. Besides, $\widetilde{\nu}_{p}$ will also typically be much larger than molecular vibrational frequencies, so that all the terms in the Raman tensor in Eq. (1) where $r$ runs over vibrational quantum numbers in the electronic ground state can be neglected relative to the contributions which involves excited electronic states, i.e., $\Delta \widetilde{\nu}_{R}=\widetilde{\nu}_{v_{b}, v_{a}} \ll \widetilde{\nu}_{p} \ll \widetilde{\nu}_{e v, g v_{a}}$. The damping constant of the excited electronic states $\gamma_{r=e}$ may also be neglected since they are of the order of magnitude as a vibrational quantum. With these assumptions and assuming that the electronic ground state is nondegenerate (which is typical), the expression for the Raman tensor in Eq. (1) can be approximated by

$$
\left(\alpha_{\rho \sigma}^{v_{a} \rightarrow v_{b}}\right)_{n o n-r e s} \cong\left\langle v_{b}\left|\hat{\alpha}_{\rho \sigma}\left(Q, \widetilde{\nu}_{v_{b}, v_{a}}, \widetilde{\nu}_{p}\right)\right| v_{a}\right\rangle
$$

where $\hat{\alpha}_{\rho \sigma}\left(Q, \widetilde{\nu}_{v_{b}, v_{a}}, \widetilde{\nu}_{p}\right)$ is the Cartesian component of the molecular transition polarizability tensor 


$$
\hat{\alpha}_{\rho \sigma}\left(Q, \widetilde{\nu}_{v_{b}, v_{a}}, \widetilde{\nu}_{p}\right)=\sum_{e \neq g} \frac{\rho_{g e}(Q) \sigma_{e g}(Q)}{\widetilde{\nu}_{e, g}-\widetilde{\nu}_{p}}+\frac{\sigma_{g e}(Q) \rho_{e g}(Q)}{\widetilde{\nu}_{e, g}+\widetilde{\nu}_{p}}
$$

To obtain Eq. (9) “the closure rule," i.e., $\hat{1}=\sum_{i}|i\rangle\langle i|$ valid for any complete set of states $|i\rangle$, has been applied to the vibrational sub-states of every electronic state, and the electronic transition moments defined above (expansion scheme 2) have been introduced. Because Eq. (9) involves a summation over all excited electronic states, a Taylor expansion of the electronic transition moments $\rho_{g e}(Q)$ and $\sigma_{e g}(Q)$ would result in too many parameters, which cannot be determined. A direct Taylor expansion of the molecular transition polarizability tensor $\hat{\alpha}_{\rho \sigma}\left(Q, \widetilde{\nu}_{v_{b}, v_{a}}, \widetilde{\nu}_{p}\right)$ is therefore more convenient in this case:

$$
\hat{\alpha}_{\rho \sigma}\left(Q, \widetilde{\nu}_{v_{b}, v_{a}}, \widetilde{\nu}_{p}\right) \cong \hat{\alpha}_{\rho \sigma}^{0}+\sum_{k=1}^{3 N-6}\left(\frac{\partial \hat{\alpha}_{\rho \sigma}}{\partial Q_{k}}\right)_{0} Q_{k}+\ldots
$$

where $Q_{k}$ is the $k^{\text {th }}$ normal coordinate. To calculate the Raman tensor, the expansion in Eq. (10) is inserted in Eq. (8), and the result is inserted in Eq. (1) in order to obtain the intensity of the Raman signal of the Raman transition $|a\rangle \rightarrow|b\rangle$.

Conclusion: It follows that the Raman signal only depends on variables related to the electronic ground state, i.e., the set of normal coordinates $Q$ and the wave numbers of the Raman bands $\widetilde{\nu}_{v_{b}, v_{a}}$, besides the wave number of the laser $\widetilde{\nu}_{p}$. However, the spectral distribution in the Raman spectra is independent of the laser wave number, which only has influence on the absolute intensity of the Raman signal. As seen from Eq. (9), all information about the individual electronically excited states is "washed out" in the calculation (and in the experimental signals), which means that we cannot obtain any information on individual excited electronic states by measuring the Raman signals under these conditions. Nonresonance Raman scattering (or RS for short) becomes therefore a vibrational spectroscopic technique just like IR and NIR. There are however two essential differences between RS and IR and NIR: first, the Raman signals are obtained by the scattering of laser light with wave numbers typically in the visible region and therefore much higher than the wave numbers of the IR and NIR photons, which are directly absorbed in IR and NIR spectroscopy. Second, since the Raman signal is controlled by a tensor instead of by a vector, the spectral selection rules of RS become different from those of IR and NIR. The selection rules for RS can easily be derived by considering Eq. (10) inserted in Eq. (8). Assuming room temperature and considering only the Stokes spectrum, it follows that Raman spectra only contain the fundamental transitions, i.e., $\left|v_{a}=0\right\rangle \rightarrow\left|v_{b}=1\right\rangle$, where the intensity is provided by the first derivative of the polarizability tensor. Besides for smaller molecules (e.g., benzene) having a high symmetry, the polarization enables one to distinguish between totally symmetric and asymmetric vibrations in the Raman spectrum. The DPR for symmetric vibrations becomes $0 \leq D P R<0.75$, which follows directly from Eq. (5) and the fact that the Raman tensor is symmetric in nonresonance (i.e., $\Sigma^{1}=0$ ). For asymmetric vibrations the $D P R=0.75$, since the tensor, because of the symmetry, has no trace (i.e., $\Sigma^{0}=0$ ) for these modes. For molecules without symmetry, all vibrations are of course in principle totally symmetric. Due to the generally narrow bandwidths of the Raman bands, Raman spectra represent in most cases a very well-defined vibrational signature of the molecule. For this reason and since the Raman signal can be measured in a reflection geometry and because no sample preparation is really needed, Raman signals are 
well suited for performing different kinds of multivariate analysis of solutions containing several molecular species.

\subsection{Vibrational resonance Raman spectroscopy}

In resonance Raman scattering, the wave number of the laser is chosen within the UV or visible absorption band of the molecule. Since the assumption

$\widetilde{\nu}_{v_{b}, v_{a}} \ll \widetilde{\nu}_{p} \ll \widetilde{\nu}_{e v, g v_{a}}$ is no longer valid, we have to go back to Eqs. (3) and (8) and insert approximations appropriate for the resonant scattering situation to be considered. First it should be noticed that resonance Raman scattering may form the basis of two different kinds of resonance Raman spectroscopy:

\section{Vibrational resonance Raman spectroscopy (VRRS)}

\section{Raman dispersion spectroscopy (RADIS) (see Section 3.3.)}

In VRRS the vibrational Raman spectrum is measured in the same way as in RS, i.e., the spectral distribution in the Raman scattered light is measured as a function of the Raman shift $\Delta \widetilde{\nu}_{R}$ with a fixed value of the wave number of the laser, but, as said, $\widetilde{\nu}_{p}$ is now chosen close to or within an electronic absorption of the molecule. The two most striking features of VRRS as compared to RS are that the intensity of the Raman signal is largely enhanced (typically with a factor of $10^{3}-10^{6}$ ) and that the intensity distribution in the vibrational spectrum is different in general. It follows from Eq. (3) that the enhancement is due to the fact that the real part in the denominators of the Raman tensor becomes small or even zero for the states being close to or in resonance with the laser. Since the state tensors associated with the resonating states dominate in the Raman intensity, the selection rules will now be determined not only by the initial and final states as in the RS but also by these state tensors. It follows from Eq. (7) that this will change the intensity distribution of the Raman signal. A further consequence is that new vibrational modes may become Raman active, but more importantly not only the fundamentals are seen in VRRS spectra, but also overtones (i.e., multiple excitations of a single normal vibration) and combination bands (i.e., multiple excitations involving several vibrations) are frequently observed. The appearance of the overtones and combination bands enables one to estimate anharmonicity constants and thereby improve the modeling of the vibrational potential function in the electronic ground state.

Larger molecules, typically biomolecules, are often colored because they contain a chromophore with high symmetry. Important examples are the metal-porphyrins, where the ring structure has the ideal symmetry of $D_{4 h}$. When the wave number of the laser is chosen within the visible absorption band of the chromophore, essentially only the ring vibrations are resonance enhanced and show up in the Raman spectra with significant intensity. Besides making the total Raman spectrum simpler, it also enables one to study a small part of a very large molecule, e.g., a protein molecule. Finally, it should be noticed that under resonance conditions, the value of the DPR may take any number from zero to infinity. This is because the Raman tensor needs no longer be symmetric but in general may have all three tensor invariants $\Sigma^{0}, \Sigma^{1}$, and $\Sigma^{2}$ different from zero. As seen from Eq. (5), the value infinity is obtained when the tensor is purely antisymmetric, i.e., when only $\Sigma^{1} \neq 0$. In the 1970s very high values of the DPR of vibrational modes were first observed experimentally in the VRRS spectra of the heme-proteins [21]. The results were interpreted as being due to the scattering by a vibration with the symmetry $a_{2 g}$ 
being associated with an antisymmetric state tensor for a resonating electronic state with $E_{u}$ symmetry in the point group $D_{4 h}$.

The resonance enhancement and the interference between the resonating state tensors are very sensitive to the magnitude of the damping constants $\gamma_{r=e v}$ of the resonating states. Since the damping is a measure of the lifetime of an excited electronic state, resonance Raman scattering may provide information about the dynamics of excited molecular states. However, to obtain this kind of information, measurement of the Raman signal at more than one laser wave number is required.

\subsection{Raman dispersion spectroscopy (RADIS)}

In RADIS the resonance Raman signal is monitored as a function of the excitation wave number. In practice a series of resonance Raman spectra are measured using either a number of discrete laser wave numbers or a tunable laser. RS, VRRS, and RADIS are illustrated in Figure 2. The figure illustrates, for a thought molecule, the changes in a series of Raman spectra obtained with increasing wave number toward resonance with an electronic state. The electronic resonance is illustrated by the UV/VIS absorption spectrum. One excitation spectrum, normally called an excitation profile (dotted curve), is obtained by plotting the intensity of a specific Raman band in the Raman spectrum versus the excitation wave number. It follows from the figure that there will be one excitation profile for each Raman band. It should be stressed that a complete RADIS experiment requires that two spectra are measured at each excitation wave number, namely, the parallel and perpendicular polarized spectra. From the polarized resolved spectra, the excitation profile can be calculated as the sum of these, but more importantly the DPR as a function of the excitation wave number can be determined. The DPR versus excitation wave number is called the polarization dispersion curve.

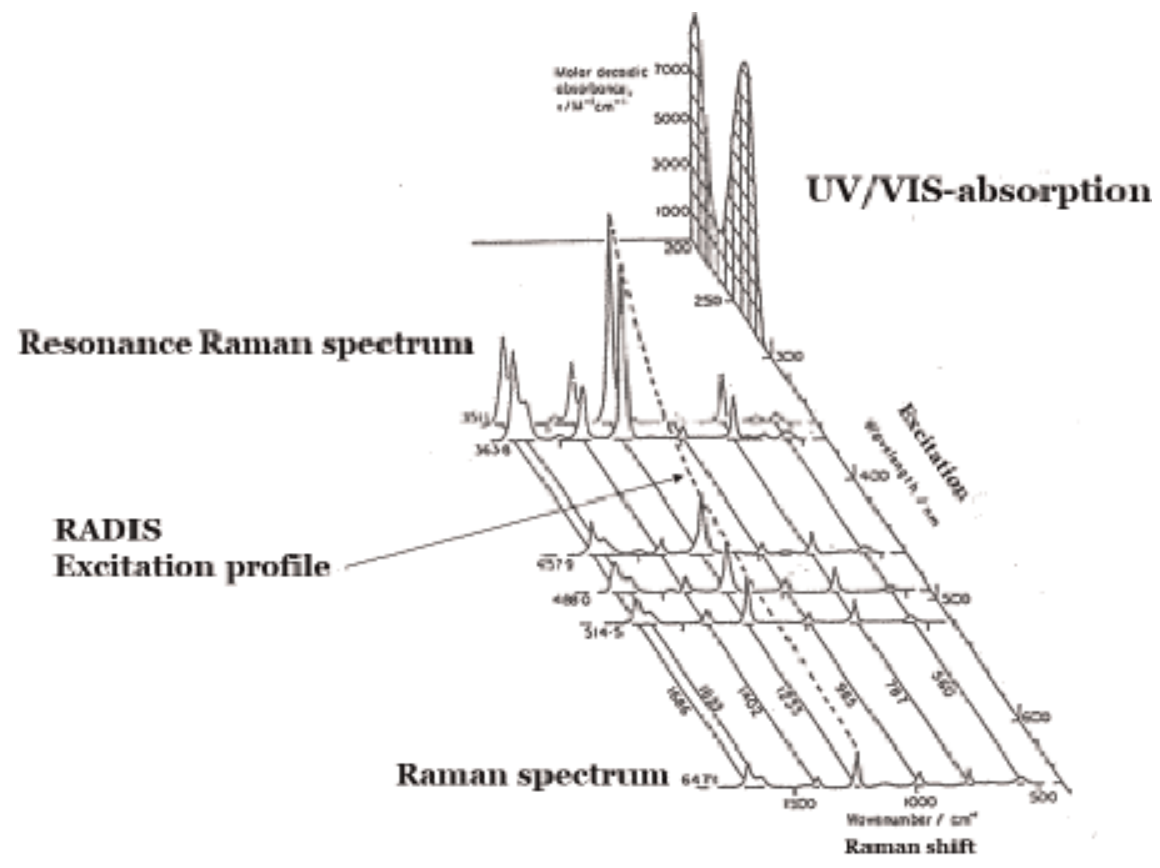

Figure 2.

Illustration of Raman, resonance Raman, and Raman dispersion (RADIS) spectroscopy. 
In a typical UV/VIS absorption experiment performed on a solution, one measures the absorbance $A$, which is related to the molar extinction coefficient $\varepsilon(\widetilde{\nu})$ via Lambert-Beers law, $A=\varepsilon(\widetilde{\nu}) C_{m} l$, where $C_{m}$ and $l$ are the molar concentration and the path length, respectively. From the application of the quantum theory, the molar extinction coefficient is found to be proportional to the spatial average of the absolute square of the transition dipole moment:

$$
\varepsilon(\widetilde{\nu}) \propto \sum_{v_{1}^{e}, v_{2}^{e}, v_{3}^{e} \ldots v_{3 N-6}^{e}}\left|\left\langle v_{1}^{e}, v_{2}^{e}, v_{3}^{e} \ldots v_{3 N-6}^{e}\left|\rho_{g e}(Q)\right| 0,0,0, \ldots\right\rangle\right|_{a v}^{2} L_{i}(\widetilde{\nu})
$$

where $v_{e} \equiv v_{1}^{e}, v_{2}^{e}, v_{3}^{e} \ldots v_{3 N-6}^{e}$ are the vibrational quantum numbers referring to the excited electronic state and to the electronic ground state, respectively. $L_{i}(\widetilde{\nu})$ is a normalized lineshape function for the $i^{t h}$ transition $|g 0\rangle \rightarrow\left|e v_{i}^{e}\right\rangle$ with a typical FWHM width of the order of magnitude as a vibrational quantum. A calculation requires that the electronic transition moment is Taylor expanded in the nuclear coordinates. Due to the superposition of the intensity of the individual transitions in Eq. (11), it follows that the vibrational fine structure in UV/VIS absorption spectra of larger molecules is not well resolved as also experienced from experiments. This is different in the RADIS spectra, where the narrow Raman bands enable the excitation profiles to be well separated experimentally (as illustrated in Figure 2). Since each excitation profile only has contributions from a single Raman-active vibration, the vibrational fine structure in the UV/ VIS absorption can be resolved. Thus, the amount of available information about the excited electronic molecular states is much larger in RADIS than in UV/VIS absorption.

It follows that while VRRS is mainly a vibrational spectroscopic technique, RADIS has more in common with electronic spectroscopy. It follows that each Raman-active vibration just plays the role of a "sensor" used to monitor the vibrational fine structure in the UV/VIS absorption spectrum.

Since the late 1980 s, a very large amount of systematic resonance Raman studies on different metal complexes [28, 29] and different metal-porphyrins [12] including heme-proteins have been performed with the goal of determining their structure, their bio-functionality, and the conditions for aggregation. In these studies both the VRRS and RADIS including both excitation profiles and polarization dispersion have been applied. Recently polarization-resolved VRRS has been combined with dynamic light scattering to study among other things the aggregation of Arenicola marina extracellular hemoglobin, which is a macromolecule with 144 heme groups instead of four as in human hemoglobin [30]. As already mentioned one great advantage of applying Raman scattering is that the technique can be performed as reflection measurements without much sample preparation. Besides, the Raman signals can be obtained through glass and other sheets of protection. Thus, Raman studies can be performed as in vivo or in situ studies. We refer to the comprehensive Raman literature on these matters for details (e.g., see [31-36]). A complete RADIS experiment may be time-consuming or in some cases impossible to carry out due to the lack of excitation lasers with the proper wave number. It may also be time-consuming to determine the correct intensity variation when the excitation wave number is changed due to changes in the scattering conditions (laser intensity and focus, laser-induced degradation of the molecule, change of the fluorescence, etc.). The application of internal standards and other means have to be introduced in order to ensure the correct experimental conditions. However, in some cases of practical interest, it is in fact possible to extract valuable information without completing a full RADIS experiment (see Sections 4.2 and 4.3). 
What Is Vibrational Raman Spectroscopy: A Vibrational or an Electronic Spectroscopic...

DOI: http://dx.doi.org/10.5772/intechopen.86838

\section{Examples}

As has been shown, vibrational RS is exclusively a vibrational spectroscopic technique like IR and NIR. However, vibrational Raman spectroscopy performed under resonance conditions may be considered as either a vibrational spectroscopic technique or as an electronic spectroscopic technique, which of the two depends on the way the experiments are performed. Three examples are briefly discussed below. For more applications, the interested reader should consult the comprehensive Raman literature [31-36].

\subsection{Example 1: perturbation of molecular symmetry}

As demonstrated in [11], the non-commuting generator approach to molecular symmetry may be applied to calculate the structure of the state tensors. Figure 3 shows an example for two vibrations in point group $D_{4 h}$. The figure also demonstrates what happens when the symmetry is lowered so that the configuration is now described in point group $D_{2 h}$. Lowering of the symmetry may be a result of a chemical reaction or may be due to a perturbation of the configuration from the planar square to a planar rectangular shape. To the left in the figure, the state tensors in $D_{4 h}$ for the Raman-active in-plane vibrations $a_{1 g}, a_{2 g}$, and $b_{1 g}$ are shown, which are written in front of the tensors. The symmetries of the two components of the resonating, degenerate electronic state with $E_{u}$ symmetry are written in the tensors on the positions, which correspond to the only nonvanishing elements. The plus and minus signs describe the numerical relations between the tensor elements. For the $a_{1 g}$ vibration, it follows that $S_{x x}^{\left|e 0=E_{u, x}\right\rangle}=S_{y y}^{\left|e 0=E_{u, y}\right\rangle}$, while for the $b_{1 g}$ vibration, we have that $S_{x x}^{\left|e 0=E_{u, x}\right\rangle}=-S_{y y}^{\left|e 0=E_{u, y}\right\rangle}$. The state tensor for the $a_{2 g}$ vibration is antisymmetric, i.e., $S_{x y}^{\left|e 0=E_{u, y}\right\rangle}=-S_{y x}^{\left|e 0=E_{u, x}\right\rangle}$. From the tensor structure, the DPR values can be calculated by using Eq. (5) and the relations given in [11] or [3]. The DPR values are written to the right side of the tensors and are seen to be constants. By correlating the symmetries of the two point groups, the symmetries of the vibrations are changed as follows: $a_{1 g} \rightarrow a_{g}, b_{1 g} \rightarrow b_{2 g}$ and $a_{2 g} \rightarrow b_{g 1}$. The state tensors for the $a_{g}, b_{1 g}$ and $b_{g 2}$ vibrations in $D_{2 h}$ are also shown in Figure 3. As before, inside the tensors the symmetries which the intermediate states must have in order

$$
\begin{array}{cc}
a_{1 g}:\left[\begin{array}{ccc}
E_{u, x} & 0 & 0 \\
0 & E_{u, y} & 0 \\
0 & 0 & 0
\end{array}\right]_{D P R=\frac{1}{8}} & a_{g g}:\left[\begin{array}{ccc}
B_{3 u} & 0 & 0 \\
0 & B_{2 u} & 0 \\
0 & 0 & B_{1 u}
\end{array}\right]_{D P R=\frac{1}{3}} \\
b_{1 g}:\left[\begin{array}{ccc}
-E_{u, x} & 0 & 0 \\
0 & E_{u, y} & 0 \\
0 & 0 & 0
\end{array}\right]_{D P R=\frac{3}{4}} & b_{2 g:}:\left[\begin{array}{ccc}
0 & 0 & B_{1 u} \\
0 & 0 & 0 \\
B_{3 u} & 0 & 0
\end{array}\right]_{D P R\left(\tilde{v}_{p}\right)} \\
a_{2 g}:\left[\begin{array}{ccc}
0 & E_{u, y} & 0 \\
-E_{u, x} & 0 & 0 \\
0 & 0 & 0
\end{array}\right]_{D P R \rightarrow \infty} & b_{1 g}:\left[\begin{array}{ccc}
0 & B_{2 u} & 0 \\
B_{3 u} & 0 & 0 \\
0 & 0 & 0
\end{array}\right]_{D P R\left(\hat{v}_{p}\right)}
\end{array}
$$

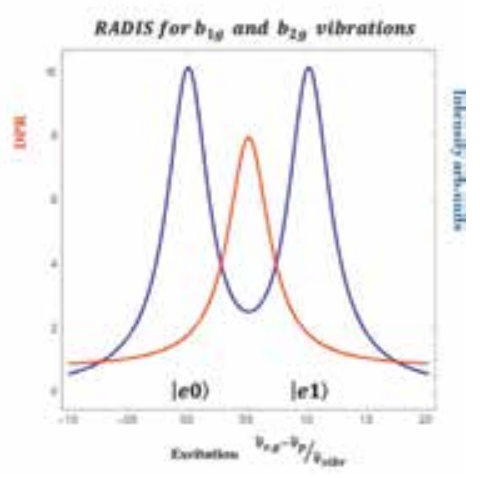

Figure 3.

(Left) Changes of the state tensors, induced by perturbation of a square planar $\left(D_{4 h}\right)$ molecular configuration into a rectangular planar $\left(D_{2 h}\right)$ configuration. (Right) Most notably is the change of the constant DPR into $D P R$ dispersion (red curve) for the $b_{1 g} \wedge b_{g 2}$ vibrations $\left(D_{2 h}\right)$. 
to give rise to any Raman signal are written. Due to the lowering of the symmetry, there are no longer numerical relations between the tensor components. Considering, e.g., the totally symmetric vibration, we see that a resonating state must have either $B_{3 u}, B_{2 u}$ or $B_{1 u}$ symmetry and that, e.g., an electronic state with $B_{3 u}$ symmetry gives rise to a tensor where only $S_{x x}^{\left|e 0=B_{3 u}\right\rangle} \neq 0$.

For the asymmetric $b_{2 g}$ vibration, the $B_{3 u}$ electronic state would result in a tensor where only the component $S_{z x}^{\left|e 0=B_{3 u}\right\rangle} \neq 0$, whereas one vibrational excitation of this vibration gives a state with the symmetry $b_{2 g} \otimes B_{3 u}=B_{1 u}$, which is seen to correspond to the transposed state tensor, i.e., only $S_{x z}^{\left|e 1=B_{1 u}\right\rangle} \neq 0$. From the power expansion of the vibronic state tensor given in Eq. (7), it may be shown that the two tensor elements satisfy the relation: $S_{z x}^{|e 0\rangle}=S_{x z}^{|e 1\rangle}$. A closer investigation shows that this relation is valid in general for asymmetric vibrations [11]. The Raman tensor is calculated by inserting the two state tensors in Eq. (3). The DPR is calculated by using Eq. (5) and the relations between the rotational invariants and the Raman tensor derived in [11]. By group theory, it can be shown that an external electronic perturbation with symmetry $B_{1 g}$ (in $D_{4 h}$ ) would result in the considered shift in the molecular configuration. As shown in Figure 3, the consequence of the symmetrylowering perturbation of the molecular configuration is that the DPR now shows a characteristic symmetric polarization dispersion with maximum half ways between the energy positions of the two states $|e 0\rangle$ and $|e 1\rangle$. The excitation profile is symmetric around the maximum of the DPR curve and has maxima at the positions of these states, i.e., at $|e 0\rangle$ and $|e 1\rangle$. In the point group $D_{4 h}$, the state tensor for the $a_{2 g}$ vibration is purely antisymmetric with a result that the DPR becomes infinity. After the perturbation, where $a_{2 g} \rightarrow b_{g 1}$, the $b_{1 g}$ state tensor is seen to have the same structure as the tensor for the $b_{2 g}$ mode, which means that the DPR dispersion curve and excitation profile also become similar. However, the energy of the state $|e 1\rangle$ will in general be different for different modes.

It follows that through the application of RADIS, it is possible to study small changes of the molecular configuration in excited electronic states and estimate the various molecular parameters influenced by these changes. As shown in numerous RRS papers on biomolecules, these structural changes, which are typically induced by minor changes in the environment of the molecule, can be studied in vivo, which is of course a major advantage [31-36].

\subsection{Example 2: noninvasive color detection using polarization dispersion}

The color of a molecular species is associated with the properties of the electronic excited states of the molecule, and in large biomolecules, it is due the presence of a chromophore being typically a metal complex. The red color of the hemeproteins, which is due to the presence of the Fe-porphyrin complex, is a well-known example. A change in color may be due to a change of the molecular configuration (distortion, aggregation) or be a result of a chemical reaction. By monitoring the color change before and after a chemical reaction, the substance concentration in solutions can be determined from the absorbance measured by a UV/VIS spectrophotometer or in the case of a solid by applying the spectrophotometer with an integrating sphere. In the literature several color detection methods have been developed for the detection of various substances. A book on color detection is in the process of publication by IntechOpen and will be published later in 2019.

In this section a reflection technique with high spectral resolution is discussed. The technique is suitable for the detection of minor color differences between 
similar molecules, i.e., molecules where a number of identical vibrations can be identified in the vibrational signature of the molecules. The method has been applied in combination with polarized resolved fluorescence to study the stability of the Ruthenium-based dye N719 [37] and to study in vitro the stability of N719 and the adsorption and desorption processes of this complex to the $\mathrm{TiO}_{2}$ substrate in dye-sensitized solar cells [38]. We refer to this paper for details.

Recently the method has also been proposed as a possible noninvasive screening technique for revealing a content of carbon monoxide in fresh tuna fish or meat. Preliminary experimental results were presented at the Raman conference ICORS 2016 in Brazil [39] and are discussed in the following.

The method is based on the presence of dispersive Raman modes combined with a small spectral difference between the visible absorption spectra of similar molecules. The idea is as follows: the resonance condition for a specific molecule in RRS depends, as we have seen above, on the difference in the wave number between the electronic absorption and the excitation laser. Due to the tensor property of resonance Raman scattering, the value of the DPR depends on this difference (polarization dispersion). Thus, a small spectral shift in the absorption will essentially be equivalent to a displacement of the polarization dispersion curve relative to the excitation wave number of the laser, as illustrated in Figure 5. The change of the DPR value at a specific wave number depends on the shape of the dispersion curve, which depends on the nature of the vibration and the Raman tensor. When the molecule has low or no symmetry, most Raman-active vibrations will be dispersive. Although the ideal symmetry of a chromophore is often high, which limits the number of dispersive modes, the real symmetry is frequently lowered due to perturbations of the chromophore, which opens up for dispersion. The heme group with the ideal symmetry $D_{4 h}$ is an example. This means that in reality the appearance of Raman modes exhibiting dispersion is quite common.

In the modified atmosphere packaging of fresh fish and meat products, the products are frequently exposed to carbon monoxide. Due to the higher binding affinity of $\mathrm{CO}$ in comparison with $\mathrm{O}_{2}, \mathrm{CO}$ replaces $\mathrm{O}_{2}$ in myoglobin in the muscle tissue with high affinity, which results in the cherry-red carboxy-myoglobin complex $M b C O$. Due to the red color and high stability of $M b C O$, the fish or meat products will appear to be more fresh and attractive for a longer time period than the unexposed products. In [40] a quantitative method for the determination of $C O$ bound to myoglobin based on visible absorption spectroscopy has been developed. Although this method has a high accuracy, it requires taking a sample from the product followed by sample preparation before the absorption spectra can be measured. Figure 4 shows a fresh tuna beef sample together with the polarizationresolved RRS spectra measured on the sample without any sample preparation but measured before and after approximately 10 minutes of exposure to $C O$ and exciting the sample with a solid-state $532 \mathrm{~nm}$ laser. The experimental DPR values estimated from six Raman modes are collected in Figure 5. Further experimental details including details on the data processing of the polarization-resolved RRS data are obtainable from the author. Figure 5 also shows, as an example, a simulation of the DPR dispersion curves for the mode at $1450 \mathrm{~cm}^{-1}$ present in the polarization-resolved RRS spectra and which has been assigned as a $b_{1 g}$ mode. The spectral shift (color shift) due to the exposure with $C O$ is $\approx 9 \mathrm{~nm}$ equivalent to $275 \mathrm{~cm}^{-1}$, estimated from measuring the diffuse reflectance of the tuna beef sample before and after exposure with $C O$ with a Lambda 900 spectrophotometer equipped with an integrating sphere. The polarization-resolved RRS data were collected by using a fiber-coupled, $180^{\circ}$ Raman microscope consisting of a modified OlympusBX60F5, a SpectraPro 2500i spectrograph (Acton) with 1200 lines $/ \mathrm{mm}$ 
grating, cooled CCD (Princeton Instr/Acton PIXIS), and $532 \mathrm{~nm} \mathrm{cw}$ laser (VentusLP532) focused with $10 \mathrm{x}$ objective with $\mathrm{NA}=0.30$. Collection optic: Same objective combined with Dichroic mirror and multimode fiber coupling to spectrograph. Av. integration time 30 seconds. No sample preparation.

First, it is noticed that the DPR values of five out the six modes indicate that they are dispersive, since all the DPR values are changed due to the exposure to $C O$. The polarization dispersion shows that the real symmetry of both $\mathrm{MbO}_{2}$ and $\mathrm{MbCO}$ must be lower than $D_{4 h}$, which would give constant values for the DPR. It is also noticed that all the DPR values are smaller than 0.75 , which indicate that the polarization dispersion is different from the one considered previously (see Figure 3). The DPR dispersion curve in Figure 5 is calculated for the $b_{1 g}$ mode at $1450 \mathrm{~cm}^{-1}$. It is obtained by adopting the procedure discussed in [11], where instead of considering the exact symmetry of the perturbed molecule (e.g., $D_{2 h}$ ) it is assumed that the molecule is really a weakly perturbed $D_{4 h}$ system. In our example it means that the state tensor relation $S_{x x}^{\left|e 0=E_{u, x}\right\rangle}=-S_{y y}^{\left|e 0=E_{u, y}\right\rangle}$ is still valid, but the degeneracy of the $\left|E_{u}\right\rangle$ state is lifted because of the perturbation, so that the two contributions to the Raman tensor now appear at slightly different energies (wave numbers). The splitting of the energy of the $\left|E_{u}\right\rangle$ state combined with the relation between the state tensors gives rise to constructive and destructive interference
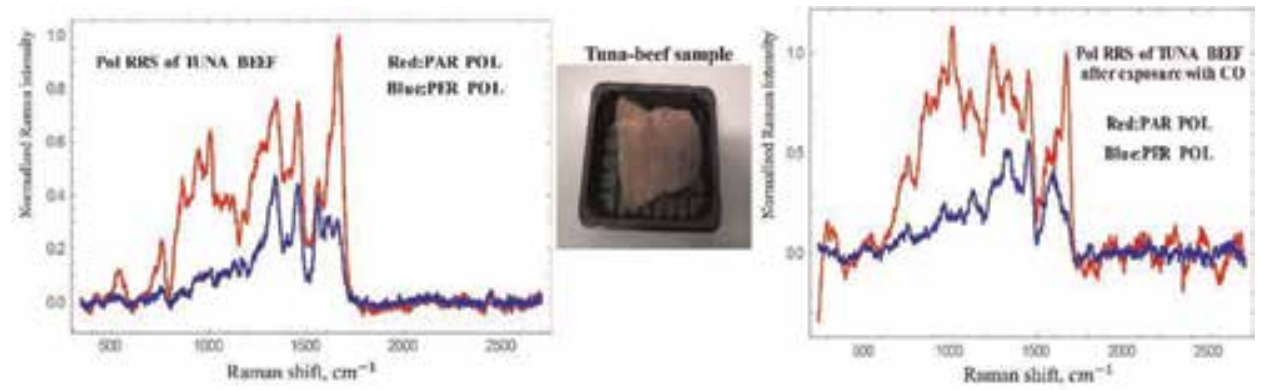

Figure 4.

Fluorescence-corrected and normalized polarization-resolved RRS spectra of the tuna beef sample measured before and after 10 minutes. Exposure with CO.

\begin{tabular}{|c|c|c|c|}
\hline \multicolumn{4}{|c|}{$\begin{array}{l}\text { Experimental DPR values } \\
\text { obtained with } 532 \mathrm{~nm} \text { excitation }\end{array}$} \\
\hline $\begin{array}{l}\text { Tuna beef } \\
\left(\mathrm{MbO}_{2}\right) \\
\text { Raman mode } \\
\mathrm{cm}^{-1}\end{array}$ & DPR & $\begin{array}{l}\text { Tuna beef after } \\
\text { exposure with } \\
\text { CO. (MbCO) } \\
\text { Raman mode } \\
\qquad \mathrm{cm}^{-1}\end{array}$ & DPR \\
\hline 1338 & 0.56 & 1332 & 0.64 \\
\hline 1399 & 0.36 & 1392 & 0.48 \\
\hline 1450 & 0.56 & 1452 & 0.66 \\
\hline 1558 & 0.76 & 1560 & 0.69 \\
\hline 1609 & 0.66 & 1609 & 0.80 \\
\hline 1658 & 0.30 & 1666 & 0.29 \\
\hline
\end{tabular}

\section{Simulation of DPR dispersion for the $1450 \mathrm{~cm}^{-1}$ Raman mode}

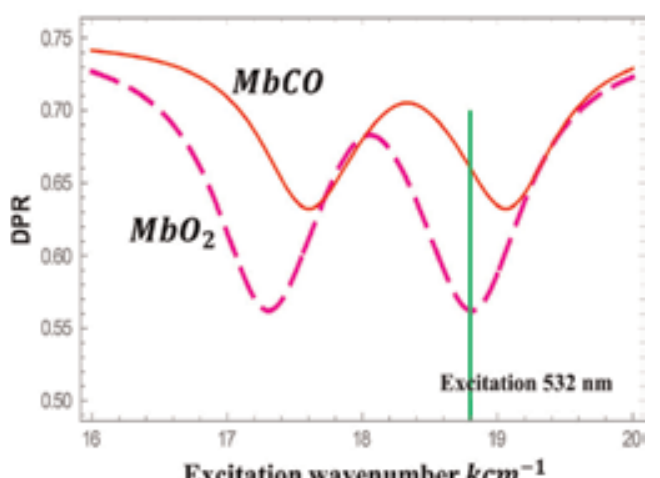

Figure 5.

Left: experimental DPR determined from the polarized RRS data in Figure 4 using a 6 mode fit (details available from author). Right: simulation and best fit of the DPR dispersion for the $b_{1 g}$ mode at $1450 \mathrm{~cm}^{-1}$ applying an "electronic interference model." 
phenomena in the Raman intensities in the resonance region. Each dispersion curve in Figure 5 is calculated by using only a single adjustable parameter, namely, the energy splitting of the $\left|E_{u}\right\rangle$ state. The energy splittings, which give the best fit to the experimental values, are as follows: $465 \mathrm{~cm}^{-1}$ and $330 \mathrm{~cm}^{-1}$ for $\mathrm{MbO}_{2}$ and $\mathrm{MbCO}$, respectively. The energy splittings are small in comparison with the vibrational wave numbers and correspond therefore to a small perturbation of the molecules away from the $D_{4 h}$ configuration. Since the small energy splittings are also comparable to the estimated bandwidths of the electronic transitions $\left(\gamma_{\left|E_{u}\right\rangle} \sim 400 \mathrm{~cm}^{-1}\right)$, they are not resolved in the visible absorption spectra, but as it follows from Figure 5, they give rise to measurable effects in the DPR dispersion. At the excitation wave number $18,797 \mathrm{~cm}^{-1}$ corresponding to excitation with the $532 \mathrm{~nm}$ laser, the change of the DPR value, induced by the exposure with $C O$, is about $10 \%$. The outcome of the experiment can be improved when the DPR data from all dispersive modes is considered. Another improvement, which would increase the reliability of the results considerably, would be a simultaneous measurement of the parallel and perpendicular polarized spectra, since this opens up for randomization and spatial averaging of the data. Simultaneous measurements of both polarizations can be obtained by modifying the excitation and collection optics in a standard Raman setup with CCD detector, in such a way that the upper and lower halves of the CCD can collect the parallel and perpendicular polarized spectra, respectively. This modification would also permit applications of polarization-resolved Raman imaging.

\subsection{Example 3: unpolarized RADIS as a source of three-way multivariate data}

In the last decade, chemometrics has become essential in the analysis of small differences of the chemical composition of samples in medical and environmental applications as well as in the food industry. Typically the chemical data are generated by different kinds of molecular spectroscopy: UV/VIS, fluorescence, NIR, IR, Raman, and others. A large number of mathematical methods have been developed and are in most cases part of the software package delivered with the spectrometer. The data from spectroscopy are in most cases two-way data set. Using vibrational Raman spectroscopy as an example, the elements $x_{i k}$ in the data matrix are as follows: $x_{i k}=I_{\text {Raman }}^{(i)}\left(\widetilde{\nu}_{k}\right)$, i.e., the Raman intensity at the wave number point $\widetilde{\nu}_{k}$ in the Raman spectrum for the $i^{\text {th }}$ sample. Typically, these data are analyzed by the application of principal component analysis (PCA). Despite the fact that PCA analysis often gives reliable results in chemical classification problems, the recent analysis of biological samples shows that in order to obtain sufficiently high recognition ratio for secure diagnostics, one has to work with very large data sets. One way is to work with three-way data, which in general has higher information density, instead of two-way data and then apply an appropriate three-way multivariate algorithm. In classification problems involving biological samples, the three-way data has been produced by combining UV/VIS absorption data with fluorescence data in the following way: the UV/VIS absorption is measured at selected wave numbers, and the fluorescence generated at these wave numbers is measured as well. Due to low spectral resolution in both these kinds of spectra, one must produce a data matrix with very high dimension, which has the consequence that a very large number of samples must be available.

In [41] a new application of RADIS has been proposed, where the coherent absorption-emission property of Raman scattering is utilized. When we compare the construction of three-way data by combining UV/VIS and fluorescence data with the RADIS in Figure 2, we see that the unpolarized RADIS data must 
automatically be born as three-way data and more importantly the spectral resolution is very high. Consequently, only few data points along the Raman shift axis $\widetilde{\nu}_{k}$ and along the excitation axis $\widetilde{\nu}_{j}\left(\widetilde{\nu}_{j}\right.$ is an excitation wave number) for small number of samples are really needed. Thus, the elements of the RADIS data matrix are given as $x_{i j k}=I_{\text {Raman }}^{(i)}\left(\widetilde{\nu}_{j}, \widetilde{\nu}_{k}\right)$. In [41] the RADIS data matrix has been analyzed by the application of a Tucker 3 multivariate model, and various classification problems have been simulated and studied with the result that only few samples $(<10)$ and few Raman lines (3-4) and few excitation wave numbers (2-3) are needed to obtain reliable results. We refer to [41] for details.

\section{Conclusions}

What is vibrational Raman spectroscopy: a vibrational or an electronic spectroscopic technique or both? Although the Raman signal reflects the vibrational motions of the molecule in the electronic ground state, our discussion shows that the answer to the question is that the Raman technique can be applied both as a vibrational and as an electronic spectroscopic technique depending on the experimental conditions chosen.

The Raman signal provides a highly resolved vibrational signature of the molecule. However, the signature depends on whether the molecular system (molecule or ion) is excited in non-resonance or in resonance with an electronic transition. In non-resonance it follows from Eqs. (9) to (10) that the Raman signal depends on the molecular polarizability tensor evaluated in the electronic ground state and not on the molecular dipole moment as in IR and NIR. In principle all fundamental vibrations in the molecule, where $\left(\frac{\partial \hat{\alpha}_{\rho \sigma}}{\partial Q_{k}}\right)_{0} \neq 0$, may contribute to the vibrational signature. The excited electronic states have no influence on the vibrational signature. The non-resonance Raman technique is therefore a genuine vibrational technique similar to IR and NIR. The main differences are that the Raman signals are measured in a different way than the IR and NIR signals and that the polarization may, for smaller and symmetric molecules, provide additional information, also for solutions.

In resonance, where the laser wave number is chosen within an electronic absorption band of the molecule, Eq. (3) shows that the state tensors being closest to resonance with the laser will contribute most to the Raman tensor and to the Raman signal (resonance enhancement). Thus, the vibrations appearing in the resonance Raman spectra are mainly those associated with the electronic absorption. As discussed in Sections 3.2 and 3.3 and illustrated in Figure 2, resonance Raman scattering may form the basis of two different kinds of resonance Raman techniques termed VRRS and RADIS. In VRRS the focus is on the vibrational Raman spectra, just like in RS, but now obtained under resonance conditions, while in RADIS the focus is on the excitation profiles and the polarization dispersion curves. In RADIS the total Raman signal and the polarization-resolved Raman signals (giving the DPR defined in Eq. (5)) for a specific Raman-active vibration are monitored as a function of the excitation wave number. The vibronic expansion of the state tensor given in Eq. (7) shows that the Raman signal in resonance is determined by the electronic transition moment of the resonating state and its derivatives and by the relations between the vibrational sub-states associated with the electronic ground state (i.e., $\left|v_{a}\right\rangle$ and $\left.\left|v_{b}\right\rangle\right)$ and the resonating electronic states (i.e., $\left|v_{e}\right\rangle$ ). Since this will change the selection rules as compared to non-resonance, the vibrational signature of a specific molecule obtained from VRRS is therefore generally different 
from the signature obtained from RS, and it depends on the specific wave number of the laser. Due to appearance of overtones and combination bands in the VRRS, the anharmonic corrections to the vibrational potential function in the electronic ground state can be estimated. It follows that VRRS is a vibrational spectroscopic technique, where the properties of the resonating states and the state tensors associated with these have an important influence on the spectral distribution.

Since the VRRS technique can be applied also as time-resolved spectroscopy, it is an attractive tool for the investigation of both the structure and dynamics of biomolecules. The main advantage of VRRS is the ability to investigate different parts of a large protein molecule by tuning the excitation wave number into the absorption band of the chromophore of interest. In a recent paper [42], the application of VRRS in the study of the structure and dynamics of various proteins is discussed. [42] gives an excellent review of this field covering both visible and UV resonance Raman as well as cw and time-resolved versions of VRRS.

The polarization properties of the resonance Raman signal are more important in resonance than in non-resonance. For example, as discussed in [43], the uniqueness of the polarization-resolved VRRS spectra combined with standard PCA chemometrics enables one to discriminate between closely related biomolecules with almost identical unpolarized VRRS spectra. The key point is that structural molecular change manifests itself through a change of the polarization of the Raman signal (DPR). The DPR defined in Eqs. (4) and (5) is an absolute quantity, which in combination with standard PCA renders the multivariate analysis insensitive to sample and experimental variations.

The discussion in Section 3.3 and the examples presented in Section 4 demonstrate that RADIS is closer to UV/visible absorption spectroscopy than it is to vibrational spectroscopy. Besides the spectral resolution is much higher enabling the vibrational fine structure of the absorption spectra to be resolved. In resonance, the interference between the state tensors, which is the origin of the sensitivity of the Raman signal with respect to changes of the molecular parameters, is restricted to those with energy denominators closest to the laser wave number. It was also demonstrated that the polarization properties of the Raman signal, expressed through the DPR, play a more important role than in non-resonance. As said already, the DPR is defined as the ratio between two Raman signals with different polarization. The interference, which can be both constructive and destructive, will in general be different in the two Raman signals depending on the wave number of the laser and the structure of the state tensors, which again is determined by the molecular symmetry and physical properties of the molecule. Section 4.1 and Figure 3 illustrate a simple example, where the molecular configuration in an electronically excited state is distorted, which, as seen, creates a significant polarization dispersion. To fully exploit the sensitivity of the DPR to changes in the molecular parameters, one must determine the polarization dispersion, i.e., one must monitor two resonance Raman spectra (the parallel and perpendicular polarized) at each laser wave number available. Traditionally the Raman spectra with different polarizations are measured in sequence. However, with CCD technology it is possible to measure the two Raman signals simultaneously, which will improve the accuracy of the DPR considerably. This requires a modification of the entrance and collection optics of a standard Raman spectrometer, so that the upper and lower halves of the CCD monitor the parallel and perpendicular polarized Raman signals, respectively $[44,45]$.

The examples discussed in Section 4.2 show that it is possible to detect a small change in color of a molecular sample by determining the change of the DPR of a dispersive Raman mode, applying only a single excitation wave number in the absorption spectrum. To be detected the color change must be due to a modification 
of the chromophore, so that the same Raman modes are present before and after the color change. Also in these examples, the resonance Raman technique performs as a kind of electronic technique.

Finally, it is shown in Section 4.3 that due to the coherent nature of the Raman process it generates automatically the so-called three-way multivariate data. This property is applied to solve chemical classification problems by using only a few (23 ) excitation wave numbers in un-polarized RADIS in combination with a threeway multivariate model. As shown, only very few samples $(<10)$ are needed, instead of the very large (500-600) number of samples required, when the visible absorption and fluorescence spectra are combined to produce the three-way data.

\section{Author details}

Søren Hassing

Institute of Chemical Engineering, Biotechnology and Environmental Technology, University of Southern Denmark, Odense, Denmark

*Address all correspondence to: sh@kbm.sdu.dk

\section{IntechOpen}

(C) 2019 The Author(s). Licensee IntechOpen. This chapter is distributed under the terms of the Creative Commons Attribution License (http://creativecommons.org/licenses/ by/3.0), which permits unrestricted use, distribution, and reproduction in any medium, provided the original work is properly cited. (cc) BY 
What Is Vibrational Raman Spectroscopy: A Vibrational or an Electronic Spectroscopic... DOI: http://dx.doi.org/10.5772/intechopen.86838

\section{References}

[1] Raman CV, Krishnan KS. A new type of secondary radiation. Nature. 1928; 121:501

[2] Raman CV. A new radiation. Indian Journal of Physics. 1928;2:387-398

[3] Long DA. The Raman Effect. Chichester: John Wiley \& Sons; 2002. ISBN: 978-0-471-49028-9

[4] Gordon JP, Zeiger HJ, Townes CH. The maser-new type of microwave amplifier, frequency standard and spectrometer. Physics Review. 1955;99: 1264-1274

[5] Schawlow AL, Townes CH. Infrared and optical masers. Physics Review. 1958;112:1940

[6] Boyle WS, Smith GE. Chargecoupled semiconductor devices. Bell System Technical Journal. 1970;49: 487-493

[7] Smith BA. Astronomical imaging applications for CCDs. In: JPL Conference on Charge-Coupled Device Technology and Applications; 1976. pp. $1235-1238$

[8] Xie L, Ling X, Fang Y, Zhang J, Liu Z. Graphene as a substrate to suppress fluorescence in resonance Raman spectroscopy. Journal of the American Chemical Society. 2009;131(29): 9890-9891

[9] Placzek G. In: Marx E, editor. Handbuch der Radiologie. Vol. 2. Leipzig: Akademische Verlagsgesellschaft; 1934. pp. 209-374

[10] Long DA. Raman Spectroscopy. London, UK: McGraw Hill; 1977

[11] Mortensen OS, Hassing S. Chapter 1: Polarization and interference phenomena in resonance Raman scattering. In: Clarke RJH, Hester RE, editors. Advances in Infrared and Raman Spectroscopy. Vol. 6. New York (US): Wiley; 1980

[12] Schweitzer-Stenner R. Polarized resonance Raman dispersion spectroscopy on metalporphyrins. Journal of Porphyrins and Phthalocyanines. 2001;5(3):198-224. DOI: 10.1002/jpp.307

[13] Siebrand W, Zgierski MZ. In: Lim C, editor. Excited States. Vol. 4. New York: Academic Press Inc.; 1979. pp. 1-134

[14] Kramers HA, Heisenberg W. Über die Streuung von Strahlung durch Atome. Zeitschrift für Physik. 1925; 31(1):681-708

[15] Weisskopf V. Zur Theorie der Resonanzfluoreszenz. Annals of Physics. 1931;9:23

[16] Goldberger KM, Watson KM. Collision Theory. New York, US: Dover Books on Physics; 2004. p. 944. ISBN-13: 978-0486435077

[17] Davydov AS. Quantum Mechanics. 1st ed. Oxford: Pergamon Press; 1965. p. 680

[18] Dirac PAM. The Principles of Quantum Mechanics. 4th ed. Clarendon Press: Oxford; 1967. 314p

[19] Mortensen OS. Structure and Bonding. Vol. 69. Berlin Heidelberg, Germany: Springer-Verlag; 1987. pp. 1-38

[20] Hassing S. In: Khan M, editor. Raman Spectroscopy and Applications. Rijeka, Croatia: InTech; 2017. pp. 143-162

[21] Spiro TG, Strekas TC. Resonance Raman spectra of hemoglobin and cytochrome c: Inverse polarization and 
vibronic scattering. Proceedings of the National Academy of Sciences of the United States of America. 1972;69:

2622-2626

[22] Shelnutt JA, Cheung LD, Chang RCC, Yu N, Felton LH. Resonance Raman spectra of metalloporphyrins. Effects of Jahn-Teller instability and nuclear distortion on excitation profiles of Stokes fundamentals. The Journal of Chemical Physics. 1977;66:3387-3398

[23] Shelnutt JA, O’Shea DC. Resonance Raman spectra of copper tetraphenylporphyrin: Effects of strong vibronic coupling on excitation profiles and the absorption spectrum. The Journal of Chemical Physics. 1978;69: 5361-5374

[24] Zgierski MZ, Pawlikowski M. Depolarization dispersion curves of resonance Raman fundamentals of metalloporphyrins and metallophthalocyanines subject to asymmetric perturbations. Chemical Physics. 1982;65:335-367

[25] Siebrand W, Zgierski MZ. Effect of solvent-induced line broadening on resonance Raman excitation profiles and depolarization ratios. The Journal of Physical Chemistry. 1982;86(24): 4718-4725

[26] Mortensen OS. Polarization dispersion in resonance Raman scattering. The effect of inhomogeneous broadening. Chemical Physics Letters. 1976;43(3):576-580

[27] Albrecht AC. On the theory of Raman intensities. The Journal of Chemical Physics. 1961;34:1476-1484

[28] Waage Jensen P, Jørgensen Bonne L. Resonance Raman spectroscopy of some iron(II) imine complexes. Journal of Molecular Structure. 1982;79:71-78

[29] Waaben Hansen P, Waage Jensen P. Vibrational studies on bisd-terpyridine-
ruthenium(II) complexes.

Spectrochimica Acta A. 1994;50:169-183

[30] Jernshøj KD, Hassing S, Olsen LF. A combination of dynamic light scattering and polarized resonance Raman scattering applied in the study of Arenicola Marina extracellular hemoglobin. The Journal of Chemical Physics. 2013;139(6):065104

[31] Lemke C, Dreybrodt W, Shelnutt J, Quirke JME, Schweitzer-Stenner R.

Polarized Raman dispersion spectroscopy probes planar and nonplanar distortions of $\mathrm{Ni}$ (II)-porphyrins with different peripheral substituents. Journal of Raman Specroscopy. 1998;29: 945-953

[32] Guthmuller J, Champagne B. Simulating and interpreting vibrational spectra of molecules: The resonant Raman spectra of rhodamine 6G. In: AIP Conference Proceedings; 2012. 1504, p. 928. doi: $10.1063 / 1.4771848$

[33] Kiewisch K, Neugebauer J, Reiher M. Selective calculation of highintensity vibrations in molecular resonance Raman spectra. The Journal of Chemical Physics. 2008;129:204103. DOI: 10.1063/1.3013351

[34] Wächtler M, Guthmuller J, Gonzalez L, Dietzek B. Analysis and characterization of coordination compounds by resonance Raman spectroscopy. Coordination Chemistry Reviews. 2012;256:1479-1508. DOI: 10.1016/j.ccr.2012.02.004

[35] Jentzen M, Ma J, Shelnutt JA. Conservation of the conformation of the porphyrin macrocycle in hemoproteins. Biophysical Journal. 1998;74:753-763

[36] Schindler J, Kupfer S, Ryan AA, Flanagan KJ, Senge MD, Dietzek B. Sterically induced distortions of nickel (II) porphyrins-Comprehensive investigation by DFT calculations and resonance Raman spectroscopy. 
What Is Vibrational Raman Spectroscopy: A Vibrational or an Electronic Spectroscopic...

DOI: http://dx.doi.org/10.5772/intechopen.86838

Coordination Chemistry Reviews. 2018; 360:1-16. DOI: 10.1016/j.ccr.2017.12.014

[37] Hassing S, Jernshøj KD, Nguyen PT, Lund T. Investigation of the stability of the ruthenium-based dye (N719) utilizing the polarization properties of dispersive Raman modes and/or of the fluorescent emission. Journal of Physical Chemistry C. 2013;117:23500-23506

[38] Hassing S, Jernshøj KD, Nguyen PT, Lund $\mathrm{T}$. In vitro polarized resonance Raman study of N719 and N719-TBP in dye sensitized solar cells. Journal of Technology Innovations in Renewable Energy. 2016;5:21-32

[39] Hassing S. Non-invasive determination of the $\mathrm{CO}$ contents in tuna fish using polarization resolved resonance Raman and/or Rayleigh spectroscopy. In: Program of XXV 2016 ICORS; Brazil; Abstract p. 61. Conference paper presented available upon request from sh@kbm.sdu.dk

[40] Smulevich G, Droghetti E, Focardi C, Coletta M, Ciaccio C, Nocentini M. A rapid spectroscopic method to detect the fraudulent treatment of tuna fish with carbon monoxide. Food Chemistry. 2007;101:1071-1077

[41] Hedegaard M, Hassing S.

Application of Raman dispersion spectroscopy in 3-way multivariate data analysis. Journal of Raman Specroscopy. 2007;39:478-489

[42] López-Peña I, Leigh BS, Schlamadinger DE, Kim JE. Insights into protein structure and dynamics by ultraviolet and visible resonance Raman spectroscopy. Biochemistry. 2015; 54(31):4770-4783. DOI: $10.1021 /$ acs. biochem.5b00514

[43] Hassing S, Jernshøj KD, Hedegaard M. Solving chemical classification problems using polarized Raman data. Journal of Raman Specroscopy. 2011;42: 21-35
[44] Kerdoncuff H, Pollard MR, Westergaard PG, Petersen JC, Lassen M. Compact and versatile laser system for polarization-sensitive stimulated Raman spectroscopy. Optics Express. 2017; 25(5):5618-5625. DOI: 10.1364/ OE. 25.005618

[45] Kiefer J. Simultaneous acquisition of the polarized and depolarized Raman signal with a single detector. Analytical Chemistry. 2017;89:5725-5728. DOI: 10.1021/acs.analchem.7b01106 



\title{
Chapter 3
}

\section{Vibrational Zero-Point Energy of Organosilicon Compounds}

\author{
Mahmoud Rahal and Abdeslam El Hajbi
}

\begin{abstract}
In this chapter, the calculation of vibrational zero-point energies (ZPEs) of organosilicon compounds is reported. An empirical formula is used. This relationship was determined by relating vibrational zero-point energy to the nature and type of bonds forming the molecule. The calculated vibrational zero-point energies for several organosilicon derivatives belonging to different categories of compounds correlate well with the reported available values. In addition, the comparison of these results with the scaled values obtained using methods of quantum chemistry (AM1, ab initio, and by a similar empirical approach) indicates the reliability of our empirical model to reproduce vibrational zero-point energy of organosilicon compounds.
\end{abstract}

Keywords: zero-point energy (ZPE), empirical ZPE, organosilicon compounds, theory-experiment correlation

\section{Introduction}

In the formulas of statistical mechanics, the vibrational zero-point energy (ZPE) constitutes the most significant term for the correction of the total energy of the molecules. ZPE (Figure 1) is a positive, additive, collective internal property and can be approximated by the harmonic formula:

$$
Z P E=\frac{R}{2} \sum_{i=1}^{3 N-6}\left(\frac{h c}{k}\right) \nu_{i}
$$

where the sum runs for the $3 \mathrm{~N}-6$ normal frequencies $\left(\nu_{\mathrm{i}}\right)$ in the case of a nonlinear molecule of $\mathrm{N}$ atoms, $R$ is the gas constant, $k$ is the Boltzmann constant, $\mathrm{c}$ is the speed of light, and $\mathrm{h}$ is the Planck constant.

Thus, for a precise calculation of the enthalpies of formation, the use of a reliable method to determine vibrational zero-point energy appears necessary. The experimental determination of the ZPE requires the knowledge of all normal vibration mode frequencies by infrared and Raman method. In certain cases [1, 2], these methods involve experimental difficulties, and they are not feasible because of the existence of overtones and combination frequencies in the molecular spectra. Also, this experimental determination becomes even more difficult for the hazardous [3-6] compounds which may be difficult to handle in air [7-9]. Otherwise, vibrational zero-point energy can be obtained by computing molecular vibrational frequencies with quantum chemistry methods [10-14]. However, such calculations 


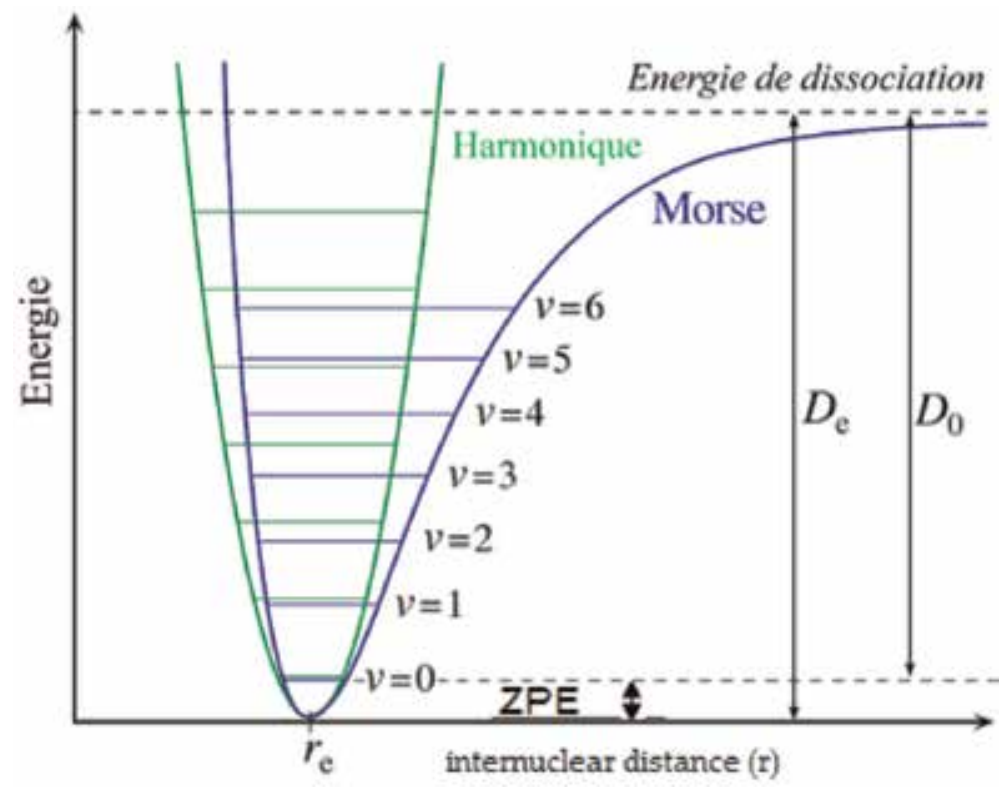

Figure 1.

The Morse potential and harmonic oscillator potential.

can be very demanding in terms of computer time and disk space for large molecules and thus in daily practice; they are not usable by the chemist and technologist. Moreover, the theoretical vibrational frequencies are generally overestimated. The neglect of anharmonicity effects in the theoretical treatment constitutes the major source of this disagreement [15]. To solve this problem, we use an empirical correction factor which depends on the method, the basis set, the property to be determined (ZPE, $\mathrm{H}_{\mathrm{vib}}(\mathrm{T}), \mathrm{S}_{\mathrm{vib}}(\mathrm{T})$, etc.), and sometimes the type of vibrations (low-frequency vibrations, high-frequency vibrations) [16]. For this reason and based on the fact that vibrational zero-point energy (ZPE) can be described with a good approximation by additivity rules [17-19], several researchers have sought to develop empirical rules to determine the vibrational $\mathrm{ZPE}$.

Thus, to estimate ZPEs of molecules without using quantum chemistry methods, several empirical rules [20-24] have been developed in recent years. These additivity rules are classified into two categories: the first methods are based on the atomic contributions, while the second one are based on the contributions of bonds or groupings. The first category takes into account each of the atoms present in a given molecule. Thus, the studied property is evaluated as the sum of the atomic contributions. In the second approach, the molecule is divided into different fragments (bonds or groups). When a fragment is present in one molecule or another, the value of its contribution remains the same.

Using the first approach based on the additivity of atomic contributions, Flanigan et al. [20] have calculated the vibrational zero-point energy (ZPE) of hydrocarbons $\mathrm{C}_{\mathrm{n}} \mathrm{H}_{\mathrm{m}}$ through the simple empirical relationship:

$$
\mathrm{ZPE}=2 \mathrm{n}+7 \mathrm{~m}(\mathrm{kcal} / \mathrm{mol})
$$

In 1985, using the least squares method, Schulman and Disch established a similar empirical relationship [25]. They determined the contributions of carbon and hydrogen atoms to estimate the vibrational zero-point energy of hydrocarbons. The relation thus obtained is written as follows: 


$$
\mathrm{ZPE}=3.88 \mathrm{n}+7.12 \mathrm{~m}-6.19(\mathrm{kcal} / \mathrm{mol})
$$

Then, this last formula has been extended to polyatomic molecular systems containing nitrogen, oxygen, chlorine, fluorine [26], bromine, sulfur [27], and silicon [28]. More recently, in order to obtain the ZPE of organophosphorus compounds, we have determined the increment of the phosphorus atom [29].

Eq. (3) takes now the form

$$
\mathrm{ZPE}=\sum_{i}^{N} N_{i} X_{i}-6.19(\mathrm{kcal} / \mathrm{mol})
$$

where $\mathrm{N}$ is the number of kinds of atom in the molecule; $\mathrm{N}_{\mathrm{i}}$ is the number of atoms of type $i$; $X_{i}$ is the increment of the atom $i$.

In this context, Grice and Politzer [1] have also developed a simple linear relationship between the ZPE and molecular stoichiometry for several organic compounds.

In 2003, Ruzsinszky et al. [30] examined the relationship between ZPE values and partial charges calculated at the functional density theory (DFT) level. The results show that atomic partial charges can be used to estimate ZPEs with high accuracy. However, this method still requires to quantum chemical calculations to estimate the ZPE of the molecules.

The sum ZPE $+\mathrm{H}(\mathrm{T})-\mathrm{H}(0)$ was studied by Fliszar et al. [13]. They found that this quantity obeyed to certain additivity rules, and they proved its correlation with the structural characteristics of the molecule, more precisely the number of atoms and the degree of branching in the case of hydrocarbons.

For the approach based on the contributions of bonds or groupings, Pitzer [31] was interested in the computation of the thermodynamic functions for gaseous hydrocarbons; he attributed an empirical value to each mode of vibration. In an n-paraffin study, Cottrell [32] found that the ZPE increases gradually with successive additions of the methylene group $\left(\mathrm{CH}_{2}\right)$. Later, Pitzer and Catalano [33] assigned the constant $17.7 \mathrm{kcal} / \mathrm{mol}$ per unit of $\mathrm{CH}_{2}$ to calculate vibrational zero-point energy of these compounds.

The empirical estimation of vibrational zero-point energies of halomethanes, ethylene, haloethylenes, methane isotopes, and benzene has been the subject of several studies by Bernstein [34-36]. He took into consideration the contributions of the internal coordinates and the interactions between them. A few years later, three empirical parameters were determined by Fujimoto and Shingu [37] to calculate the ZPEs of hydrocarbons with a precision similar to that of Bernstein, which are the contributions of $\mathrm{C}-\mathrm{C}$ bonds, $\mathrm{C}-\mathrm{H}$ bonds, and carbon chain.

In 1980, based on a system of harmonic oscillators, Oi and his collaborators published some papers [17-19] which describe the theoretical foundation showing the additivity of ZPE estimates.

Still in the framework of the estimates based on the contributions of the bonds or groupings, we established in 2001 an original empirical relation [38] which makes it possible to calculate the ZPE of the organic compounds. This rule was determined by linking the ZPE to the nature and type of bonds forming the molecular system. The established empirical formula is as follows:

$$
\mathrm{ZPE}(\mathrm{emp} .)=\sum_{\mathrm{i}}^{\mathrm{P}} \mathrm{N}_{\mathrm{i}} \times B \mathrm{C}_{\mathrm{i}}-2.09(\mathrm{kcal} / \mathrm{mol})
$$

where $\mathrm{P}$ is the number of bonds in the molecule; $\mathrm{N}_{\mathrm{i}}$ is the number of bonds of type $i ; C_{i}$ is the contribution of the bond $i$ to the $Z P E$. 
This established empirical relationship also makes it possible to calculate the vibrational zero-point energies of the aromatic derivatives with accuracy, provided that the empirical values are adjusted by the following equation:

$$
\mathrm{ZPE}=1.08 . \mathrm{ZPE}(\text { empirical })-1.07(\mathrm{kcal} / \mathrm{mol})
$$

This adjustment can be explained by the fact that in aromatic compounds, the bonds in the aromatic nucleus are all identical because of the conjugation, whereas in our model it has been assumed that there are three $\mathrm{C}-\mathrm{C}$ single bonds and three $\mathrm{C}=\mathrm{C}$ double bonds.

Eq. (5) was used to calculate the ZPEs of several organic compounds belonging to different classes of compounds (hydrocarbons, oxygen compounds, nitrogen compounds, chlorinated compounds, brominated compounds, fluorinated compounds, sulfur compounds, aromatic compounds, etc.). Thus, the contributions of the bonds $\mathrm{C}-\mathrm{H}, \mathrm{N}-\mathrm{H}, \mathrm{O}-\mathrm{H}, \mathrm{S}-\mathrm{H}, \mathrm{C}-\mathrm{O}, \mathrm{C}-\mathrm{C}, \mathrm{C}-\mathrm{N}, \mathrm{C}-\mathrm{S}, \mathrm{N}-\mathrm{N}, \mathrm{C}-\mathrm{F}, \mathrm{C}-\mathrm{Cl}, \mathrm{C}=\mathrm{C}$, $\mathrm{C}=\mathrm{N}, \mathrm{C}=\mathrm{O}, \mathrm{C}=\mathrm{S}, \mathrm{C} \equiv \mathrm{C}$, and $\mathrm{C} \equiv \mathrm{N}$ have been determined. To extend our model to brominated compounds, we have determined in 2004 the contribution of the $\mathrm{C}-\mathrm{Br}$ bond [39] and incorporated it into our empirical formula. The calculated vibrational zero-point energies for 38 compounds containing this bond $(\mathrm{C}-\mathrm{Br})$ correlate well with experimental values. In addition, we have extended the field of application of this empirical model to organophosphorus compounds (III). The bond contributions of $\mathrm{P}-\mathrm{F}, \mathrm{P}-\mathrm{C}, \mathrm{P}-\mathrm{H}, \mathrm{P}-\mathrm{Cl}, \mathrm{P}-\mathrm{S}, \mathrm{P}-\mathrm{N}$, and $\mathrm{P}-\mathrm{O}$ were determined [29]. The results obtained for 101 chemical systems containing these bonds are in good agreement with experimental values. The estimated ZPEs were compared with the results obtained by application of quantum chemistry methods at the level ab initio ( $\mathrm{HF} /$ $\left.6-31 \mathrm{G}^{*}\right)$ and DFT(B3LYP/6-31G $)$, in all cases with satisfactory results.

More recently [40], to calculate vibrational zero-point energies (ZPEs) of organophosphorus compounds $(\mathrm{V})$, we determined the contributions of the bonds $\mathrm{P}=\mathrm{O}$ and $\mathrm{P}=\mathrm{S}$ and incorporated them into our empirical formula. Comparison of the results obtained for more than 80 organophosphorus compounds (V) with the reported values and with those obtained by ab initio (HF/6-31G ${ }^{*}$ ) and $\mathrm{DFT}\left(\mathrm{B} 3 \mathrm{LYP} / 6-31 \mathrm{G}^{*}\right)$ shows the reliability of the empirical approach.

In this chapter, we describe the results obtained in the case of organosilicon compounds. We present the values obtained for the contributions of the $\mathrm{Si}-\mathrm{H}$, $\mathrm{Si}-\mathrm{C}, \mathrm{Si}-\mathrm{Cl}, \mathrm{Si}-\mathrm{O}$, and $\mathrm{Si}-\mathrm{Si}$ bonds which make it possible to calculate the vibrational zero-point energies of the silicon compounds. The results thus obtained are compared firstly with the available experimental values, secondly to the values obtained by the methods of the quantum chemistry at the semiempirical (AM1) and $\mathrm{DFT}\left(\mathrm{B} 3 \mathrm{LYP} / 6-31 \mathrm{G}^{*}\right)$ level, and finally to the results derived from a similar approach based on simple atom additivity.

\section{Computational methods}

The theoretical calculations were performed at the semiempirical [41] and density functional theory $[42,43]$ levels using, respectively, the AM1 method and B3LYP [44-46] with 6-31G ${ }^{*}$ basis set which were implemented in the Gaussian03W program $[47,48]$. The molecular geometries were optimized without any symmetry constraints, and the harmonic frequencies were calculated to ensure that the structures really corresponded to a true local minimum energy on the potential energy surface in the first time and to determine the vibrational zero-point energies in the second time. 


\section{Results and discussion}

\subsection{Estimation based on simple bond additivity}

Using the least squares method, we have determined the contributions of the $\mathrm{Si}-\mathrm{H}, \mathrm{Si}-\mathrm{C}, \mathrm{Si}-\mathrm{Cl}, \mathrm{Si}-\mathrm{O}$, and $\mathrm{Si}-\mathrm{Si}$ bond by correlating, for a population of molecules, the values of the vibrational zero-point energies obtained experimentally and those obtained by Eq. (5). The values of the contributions obtained for the studied bonds and those already established [29, 38-40, 49] are given in Table 1.

To test the reliability of the extended empirical model, we applied it to 91 silicon compounds different from those used in the compilation of contributions. This group of molecules contains different classes such as silanes, siloxanes, chlorosilanes, silyl ethers, silanols, silyl chlorides, cyclic organosilicon, and aromatic organosilicon.

The ZPEs obtained are recorded in Table 2 . These results show a very good agreement between the calculated and the experimental values. Indeed, the average error is of the order of $1.51 \mathrm{kcal} / \mathrm{mol}$ for the 91 molecular systems for which experimental or ab initio $\left(\mathrm{HF} / 6-31 \mathrm{G}^{*}\right)$ data are available. However, the ZPEs calculated for 3-phenyl-1,3-thiasilacyclohexane, 1-phenyl-1-silacyclohexane, 3-methyl-3phenyl-1,3-thiasilacyclohexane, 1-methyl-1-phenyl-1-silacyclohexane, and $\left(\mathrm{C}_{6} \mathrm{H}_{5}\right)_{3} \mathrm{SiOH}$ are underestimated.

This is due to the presence of the aromatic ring in these organosilicon compounds [29, 38-40]. Nevertheless, the adjustment of empirical ZPE values by Eq. (6) leads to a decrease of the mean error which becomes $1.02 \mathrm{kcal} / \mathrm{mol}$ (i.e., $1.98 \%$ ).

The curve of correlation between the experimental and empirical values (Figure 2) appears very satisfactory, the slope is close to unity (0.99), the correlation coefficient is equal to 0.9994 , and standard deviation is 1.2. The statistical data concerning the regression curves $\mathrm{ZPE}_{\exp }=\mathrm{aZPE}$ theor $+\mathrm{b}$ and $\mathrm{ZPE}_{\text {exp }}=\mathrm{aZPE}$ theor are summarized in Table 3. The use of this data for the adjustment of the empirical values of vibrational zero-point energies (ZPEs) reduces the mean error to $0.9 \mathrm{kcal} / \mathrm{mol}$.

\subsection{Estimation based on simple atom additivity}

In order to be able to compare the results obtained by the application of the empirical formula based on bond contribution additivity (Eq. (5)) to those obtained by the approach based on atomic contribution additivity, we have grouped in Table 2 the values of vibrational zero-point energies computed with the extended rule of Schulman and Disch (Eq. (4)). The increment of the silicon atom was calculated by AbdulHussain and Fleifel [28]. The value of this increment is shown in Table 4, with those previously published [25-29] for the atoms $\mathrm{H}, \mathrm{C}, \mathrm{O}, \mathrm{N}, \mathrm{Cl}, \mathrm{F}$, $\mathrm{Br}, \mathrm{S}$, and $\mathrm{P}$. Note that the formula of Schulman and Disch was established on the basis that the structural isomers of organic compounds have almost the same value of ZPE. However, the difference can reach $4 \mathrm{kcal} / \mathrm{mol}$ [9]. The results obtained by the method based on the additivity of the atomic contributions show, for the 91 silicon compounds, an average error of 2.53 (6.9\%). The correlation between the experimental and calculated values by the Schulman-Disch extended formula is shown in Figure 3. The statistical parameters obtained in this case are a correlation coefficient of 0.9972 , a slope of 0.95 , and a standard deviation of 2.45 . Using the regression curves (Table 3 ) for adjusting the calculated values permits to reduce the 


\begin{tabular}{|c|c|c|}
\hline Bond & Bond contribution $\left(\mathrm{BC}_{\mathbf{i}}\right)$ & Ref. \\
\hline $\mathrm{C}-\mathrm{H}$ & 7.5877 & [38] \\
\hline $\mathrm{N}-\mathrm{H}$ & 7.2013 & [38] \\
\hline $\mathrm{O}-\mathrm{H}$ & 7.2964 & [38] \\
\hline $\mathrm{S}-\mathrm{H}$ & 5.6921 & [38] \\
\hline $\mathrm{C}-\mathrm{O}$ & 2.6985 & [38] \\
\hline $\mathrm{C}-\mathrm{C}$ & 2.0751 & [38] \\
\hline $\mathrm{C}-\mathrm{N}$ & 4.1409 & [38] \\
\hline $\mathrm{C}-\mathrm{S}$ & 1.4403 & {$[38]$} \\
\hline $\mathrm{N}-\mathrm{N}$ & 6.8372 & [38] \\
\hline $\mathrm{C}-\mathrm{F}$ & 3.3078 & {$[38]$} \\
\hline $\mathrm{C}-\mathrm{Cl}$ & 2.2051 & {$[38]$} \\
\hline $\mathrm{C}=\mathrm{C}$ & 2.6501 & [38] \\
\hline $\mathrm{C}=\mathrm{N}$ & 3.8852 & {$[38]$} \\
\hline $\mathrm{C}=\mathrm{O}$ & 3.9343 & {$[38]$} \\
\hline $\mathrm{C}=\mathrm{S}$ & 2.7319 & [38] \\
\hline $\mathrm{C} \equiv \mathrm{C}$ & 4.4125 & {$[38]$} \\
\hline $\mathrm{C} \equiv \mathrm{N}$ & 4.8169 & [38] \\
\hline $\mathrm{C}-\mathrm{Br}$ & 1.9837 & [39] \\
\hline $\mathrm{Si}-\mathrm{H}$ & 5.8011 & [49] \\
\hline $\mathrm{Si}-\mathrm{C}$ & 0.3593 & [49] \\
\hline $\mathrm{Si}-\mathrm{Cl}$ & 1.7690 & [49] \\
\hline $\mathrm{Si}-\mathrm{O}$ & 1.3335 & [49] \\
\hline $\mathrm{Si}-\mathrm{Si}$ & -1.4548 & [49] \\
\hline $\mathrm{P}-\mathrm{H}$ & 6.6486 & [29] \\
\hline $\mathrm{P}-\mathrm{C}$ & 1.4190 & [29] \\
\hline $\mathrm{P}-\mathrm{O}$ & 1.8406 & [29] \\
\hline $\mathrm{P}-\mathrm{Cl}$ & 1.6717 & [29] \\
\hline $\mathrm{P}-\mathrm{N}$ & 0.9873 & [29] \\
\hline $\mathrm{P}-\mathrm{F}$ & 2.1507 & [29] \\
\hline $\mathrm{P}-\mathrm{S}$ & 1.5424 & [29] \\
\hline $\mathrm{P}=\mathrm{O}$ & 2.4032 & [40] \\
\hline $\mathrm{P}=\mathrm{S}$ & 0.6131 & [40] \\
\hline
\end{tabular}

Table 1.

Bond contributions to ZPE (in $\mathrm{kcal} / \mathrm{mol}$ ).

mean error to $1.68(4.0 \%)$ if the intercept is different from $0(b \neq 0)$ to $2.28(7.0 \%)$ if $\mathrm{b}=0$. These results are slightly less good than those obtained by our approach.

\subsection{Quantum chemical calculations}

To compare the results obtained by our empirical relationship with those obtained by quantum chemistry methods, we have calculated vibrational zero-point 
Vibrational Zero-Point Energy of Organosilicon Compounds DOI: http://dx.doi.org/10.5772/intechopen.87021

\begin{tabular}{|c|c|c|c|c|c|c|}
\hline \multirow[t]{2}{*}{ Compound } & \multicolumn{5}{|c|}{ ZPE (kcal/mol) } & \multirow[b]{2}{*}{ Ref. } \\
\hline & Exp. & Eq. $(5)^{a}$ & $A M 1^{b}$ & $\begin{array}{l}\text { B3LYP/ } \\
6-31 G^{* c}\end{array}$ & Eq. $(4)^{d}$ & \\
\hline $\mathrm{SiHCl}_{3}$ & 9.29 & 9.02 & 7.26 & 8.54 & 4.08 & [50] \\
\hline $\mathrm{SiH}_{3} \mathrm{Cl}$ & 17.01 & 17.08 & 14.50 & 15.88 & 13.88 & {$[51]$} \\
\hline $\mathrm{SiH}_{2} \mathrm{Cl}_{2}$ & 13.31 & 13.05 & 11.00 & 12.42 & 8.98 & {$[51]$} \\
\hline $\mathrm{SiH}_{4}$ & 19.90 & 21.11 & 17.71 & 18.87 & 18.78 & {$[52]$} \\
\hline $\mathrm{C}_{4} \mathrm{H}_{12} \mathrm{Si}$ (diethylsilane) & 90.68 & 90.26 & 87.36 & 89.65 & 91.26 & {$[52]$} \\
\hline $\mathrm{C}_{6} \mathrm{H}_{16} \mathrm{Si}$ (triethylsilane) & 125.98 & 124.83 & 121.78 & 124.68 & 127.50 & {$[52]$} \\
\hline $\mathrm{C}_{8} \mathrm{H}_{20} \mathrm{Si}$ (tetraethylsilane) & 161.23 & 159.40 & 156.08 & 159.52 & 163.74 & {$[52]$} \\
\hline $\mathrm{CH}_{4} \mathrm{SiCl}_{2}$ (dichloromethylsilane) & 27.09 & 27.67 & 25.27 & 26.67 & 27.10 & {$[53]$} \\
\hline $\mathrm{CH}_{5} \mathrm{SiCl}$ (chloromethylsilane) & 32.48 & 34.40 & 30.62 & 33.60 & 32.00 & {$[53]$} \\
\hline $\mathrm{CH}_{6} \mathrm{Si}$ (methyl silane) & 37.21 & 38.44 & 35.51 & 36.99 & 36.90 & [54] \\
\hline $\mathrm{C}_{3} \mathrm{H}_{6} \mathrm{Si}$ (1-silylpropyne) & 43.96 & 42.85 & 42.71 & 43.64 & 44.66 & [55] \\
\hline $\begin{array}{l}\mathrm{CH}_{2} \mathrm{Br}\left(\mathrm{CH}_{3}\right)_{2} \mathrm{SiH} \text { (bromomethyl dimethyl } \\
\text { silane) }\end{array}$ & 66.33 & 67.47 & 64.75 & 66.89 & 67.62 & {$[56]$} \\
\hline $\mathrm{H}_{3} \mathrm{SiSiH}_{3}$ & 30.08 & 31.26 & 27.27 & 29.69 & 29.51 & {$[57]$} \\
\hline $\mathrm{C}_{11} \mathrm{H}_{16} \mathrm{Si}$ (1-phenyl-1-silacyclohexane) & 151.56 & $151.30^{\mathrm{e}}$ & 144.93 & 145.51 & 146.90 & {$[58]$} \\
\hline $\mathrm{C}_{10} \mathrm{H}_{14} \mathrm{SiS}$ (3-phenyl-1,3-thiasilacyclohexane) & 134.31 & $133.54^{\mathrm{e}}$ & 127.60 & 137.91 & 130.65 & {$[58]$} \\
\hline $\begin{array}{l}\mathrm{C}_{12} \mathrm{H}_{18} \mathrm{Si} \text { (1-methyl-1-phenyl-1- } \\
\text { silacyclohexane) }\end{array}$ & 169.58 & $170.00^{\mathrm{e}}$ & 161.97 & 162.84 & 165.02 & {$[58]$} \\
\hline $\begin{array}{l}\mathrm{C}_{11} \mathrm{H}_{16} \mathrm{SiS} \text { (3-methyl-3-phenyl-1,3- } \\
\text { thiasilacyclohexane) }\end{array}$ & 152.32 & $152.24^{\mathrm{e}}$ & 144.66 & 146.27 & 148.77 & {$[58]$} \\
\hline $\mathrm{H}_{2} \mathrm{ClSiSiH}_{3}$ & 27.48 & 27.23 & 23.89 & 26.34 & 24.61 & [59] \\
\hline $\mathrm{HCl}_{2} \mathrm{SiSiH}_{3}$ & 23.63 & 23.20 & 20.29 & 22.63 & 19.71 & [59] \\
\hline $\mathrm{H}_{2} \mathrm{ClSiSiH}_{2} \mathrm{Cl}$ & 23.94 & 23.20 & 20.64 & 22.93 & 19.71 & [59] \\
\hline $\mathrm{Cl}_{3} \mathrm{SiSiH}_{3}$ & 19.47 & 19.17 & 16.53 & 18.62 & 14.81 & [59] \\
\hline $\mathrm{HCl}_{2} \mathrm{SiSiHCl}_{2}$ & 16.09 & 15.13 & 13.29 & 15.43 & 9.91 & [59] \\
\hline $\mathrm{Cl}_{3} \mathrm{SiSiH}_{2} \mathrm{Cl}$ & 15.90 & 15.13 & 13.14 & 15.18 & 9.91 & [59] \\
\hline $\mathrm{Cl}_{3} \mathrm{SiSiHCl}_{2}$ & 11.95 & 11.10 & 9.56 & 11.37 & 5.01 & [59] \\
\hline $\mathrm{Cl}_{3} \mathrm{SiSiCl}_{3}$ & 7.77 & 7.07 & 5.85 & 7.31 & 0.11 & [59] \\
\hline $\mathrm{Si}_{4} \mathrm{H}_{10}$ (n-butasilane) & 50.50 & 51.56 & 46.54 & 50.82 & 50.97 & {$[60]$} \\
\hline $\mathrm{Si}_{5} \mathrm{H}_{12}$ (n-pentasilane) & 60.93 & 61.70 & 56.14 & 61.23 & 61.70 & [60] \\
\hline $\mathrm{Si}_{6} \mathrm{H}_{14}$ (n-hexasilane) & 71.39 & 71.85 & 65.80 & 71.85 & 72.43 & {$[60]$} \\
\hline $\mathrm{Si}_{7} \mathrm{H}_{16}$ (n-heptasilane) & 81.85 & 82.00 & 81.57 & 82.43 & 83.16 & {$[60]$} \\
\hline $\mathrm{Si}_{8} \mathrm{H}_{18}$ (n-octasilane) & 92.54 & 92.15 & 85.11 & 92.90 & 93.89 & {$[60]$} \\
\hline $\mathrm{Si}_{9} \mathrm{H}_{20}$ (n-nonasilane) & 102.75 & 102.29 & 94.71 & 103.47 & 104.62 & {$[60]$} \\
\hline $\mathrm{Si}_{10} \mathrm{H}_{22}$ (n-decasilane) & 113.33 & 112.44 & 104.26 & 114.00 & 115.35 & [60] \\
\hline $\mathrm{C}_{2} \mathrm{H}_{5} \mathrm{SiCl}$ (gauche vinyl silyl chloride) & 37.31 & 37.05 & 35.75 & 37.14 & 35.88 & [61] \\
\hline $\mathrm{C}_{2} \mathrm{H}_{5} \mathrm{SiCl}$ (cis vinyl silyl chloride) & 37.31 & 35.70 & 34.19 & 37.14 & 35.88 & {$[61]$} \\
\hline $\begin{array}{l}\mathrm{C}_{2} \mathrm{H}_{6} \mathrm{SiCl}_{2} \text { (2-chloroethylsilyl chloride } \\
\text { C-gauche-Si-trans }(\mathrm{Gt}) \text { ) }\end{array}$ & 46.71 & 46.27 & 43.99 & 46.00 & 45.22 & [62] \\
\hline $\begin{array}{l}\mathrm{C}_{2} \mathrm{H}_{6} \mathrm{SiCl}_{2} \text { (2-chloroethylsilyl chloride } \\
\text { C-trans-Si-trans }(\mathrm{Tt}) \text { ) }\end{array}$ & 46.62 & 46.27 & 44.01 & 46.03 & 45.22 & [62] \\
\hline
\end{tabular}




\begin{tabular}{|c|c|c|c|c|c|c|}
\hline \multirow[t]{2}{*}{ Compound } & \multicolumn{5}{|c|}{$\mathrm{ZPE}(\mathrm{kcal} / \mathrm{mol})$} & \multirow[b]{2}{*}{ Ref. } \\
\hline & Exp. & Eq. $(5)^{a}$ & AM1 $^{\mathbf{b}}$ & $\begin{array}{l}\text { B3LYP/ } \\
6-31 G^{*} \mathrm{c}\end{array}$ & Eq. $(4)^{d}$ & \\
\hline $\begin{array}{l}\mathrm{C}_{2} \mathrm{H}_{6} \mathrm{SiCl}_{2} \text { (2-chloroethylsilyl chloride } \\
\text { C-trans-Si-gauche }(\mathrm{Tg}) \text { ) }\end{array}$ & 46.46 & 46.27 & 44.30 & 46.07 & 45.22 & {$[61]$} \\
\hline $\mathrm{C}_{2} \mathrm{H}_{7} \mathrm{SiCl}$ (1-chloroethylsilane) & 49.70 & 50.30 & 47.41 & 49.27 & 50.12 & [63] \\
\hline $\mathrm{C}_{2} \mathrm{H}_{7} \mathrm{SiCl}$ (gauche ethyl chlorosilane) & 51.38 & 51.65 & 49.18 & 51.11 & 50.12 & [64] \\
\hline $\mathrm{C}_{2} \mathrm{H}_{7} \mathrm{SiCl}$ (trans ethyl chlorosilane) & 51.42 & 51.65 & 49.19 & 51.10 & 50.12 & [65] \\
\hline $\mathrm{H}_{3} \mathrm{SiOH}$ & 24.21 & 23.94 & 21.77 & 23.22 & 22.18 & [66] \\
\hline $\mathrm{C}_{3} \mathrm{H}_{6} \mathrm{Cl}_{2} \mathrm{Si}$ (1,1-dichlorosilacyclobutane) & 49.65 & 51.84 & 51.55 & 52.36 & 49.10 & {$[67]$} \\
\hline $\mathrm{C}_{2} \mathrm{H}_{8} \mathrm{Si}$ (ethylsilane) & 54.79 & 55.69 & 52.75 & 54.54 & 55.02 & [68] \\
\hline $\mathrm{C}_{3} \mathrm{H}_{9} \mathrm{SiCl}$ (chlorotrimethylsilane) & 68.16 & 69.05 & 66.13 & 67.91 & 68.24 & [69] \\
\hline $\mathrm{C}_{3} \mathrm{H}_{10} \mathrm{Si}$ (trimethylsilane) & 71.95 & 73.08 & 70.20 & 71.86 & 73.14 & [69] \\
\hline $\mathrm{C}_{4} \mathrm{H}_{12} \mathrm{OSi}$ (methoxytrimethylsilane) & 93.41 & 94.07 & 90.81 & 92.94 & 94.66 & [69] \\
\hline$\left(\mathrm{CH}_{3}\right)_{3} \mathrm{SiOH}$ (trimethylsilanol) & 75.35 & 75.91 & 73.33 & 75.47 & 73.14 & [69] \\
\hline $\mathrm{C}_{3} \mathrm{H}_{10} \mathrm{Si}$ (gauche-n-propylsilane) & 72.29 & 72.94 & 70.00 & 71.89 & 73.14 & [70] \\
\hline $\mathrm{C}_{3} \mathrm{H}_{10} \mathrm{Si}$ (anti-n-propylsilane) & 72.07 & 72.94 & 70.00 & 71.78 & 73.14 & [70] \\
\hline $\mathrm{C}_{3} \mathrm{H}_{10} \mathrm{Si}$ (trans ethylmethylsilane) & 72.14 & 73.01 & 70.21 & 72.07 & 73.14 & {$[71]$} \\
\hline $\mathrm{C}_{3} \mathrm{H}_{10} \mathrm{Si}$ (gauche ethylmethylsilane) & 72.27 & 73.01 & 70.30 & 72.20 & 73.14 & {$[71]$} \\
\hline $\mathrm{C}_{4} \mathrm{H}_{10} \mathrm{Si}$ (cis methylsilylcyclopropane) & 76.81 & 77.09 & 74.07 & 75.51 & 77.02 & [72] \\
\hline $\mathrm{C}_{4} \mathrm{H}_{10} \mathrm{Si}$ (gauche methylsilylcyclopropane) & 76.44 & 77.09 & 74.10 & 75.45 & 77.02 & [72] \\
\hline $\mathrm{C}_{3} \mathrm{H}_{12} \mathrm{Si}_{2}$ (1,1,1-trimethyldisilane) & 82.56 & 83.23 & 79.69 & 82.38 & 83.87 & [73] \\
\hline $\mathrm{C}_{5} \mathrm{H}_{12} \mathrm{Si}$ (cyclopentylsilane) & 94.60 & 94.34 & 93.21 & 94.05 & 95.14 & [74] \\
\hline $\begin{array}{l}\mathrm{C}_{6} \mathrm{H}_{14} \mathrm{Si} \text { (cyclohexyl silane (chair- } \\
\text { equatorial)) }\end{array}$ & 112.15 & 111.59 & 110.54 & 111.83 & 113.26 & [75] \\
\hline $\mathrm{C}_{6} \mathrm{H}_{14} \mathrm{Si}$ (cyclohexyl silane (chair-axial)) & 112.93 & 111.59 & 110.54 & 112.00 & 113.26 & [75] \\
\hline $\mathrm{C}_{3} \mathrm{H}_{8} \mathrm{Si}$ (allylsilane) & 57.75 & 58.34 & 56.22 & 57.51 & 58.90 & [76] \\
\hline $\begin{array}{l}\mathrm{C}_{3} \mathrm{H}_{8} \mathrm{SiCl}_{2} \text { (anti dichloromethyldimethyl } \\
\text { silane) }\end{array}$ & 62.42 & 62.31 & 59.76 & 61.78 & 63.34 & {$[77]$} \\
\hline $\begin{array}{l}\mathrm{C}_{3} \mathrm{H}_{8} \mathrm{SiCl}_{2} \text { (gauche dichloromethyldimethyl } \\
\text { silane) }\end{array}$ & 61.73 & 62.31 & 59.78 & 61.75 & 63.34 & {$[77]$} \\
\hline $\mathrm{C}_{3} \mathrm{H}_{7} \mathrm{SiCl}$ (methylvinyl silyl chloride) & 54.48 & 54.38 & 52.92 & 54.55 & 54.00 & [78] \\
\hline$\left(\mathrm{H}_{3} \mathrm{Si}\right)_{2} \mathrm{CCH}_{2}$ & 49.65 & 51.26 & 47.92 & 57.01 & 51.51 & $* *$ \\
\hline$\left(\left(\mathrm{CH}_{3}\right)_{3} \mathrm{Si}\right)_{2} \mathrm{CCH}_{2}$ & 152.56 & 155.19 & 151.46 & 155.08 & 160.23 & $* *$ \\
\hline $\mathrm{C}_{5} \mathrm{H}_{12} \mathrm{Si}$ (1,1-dimethyl-1-silacyclobutane) & 93.33 & 94.55 & 93.48 & 94.57 & 95.14 & $* *$ \\
\hline $\mathrm{C}_{3} \mathrm{H}_{8} \mathrm{Si}$ (silacyclobutane) & 59.06 & 59.91 & 59.25 & 59.71 & 58.90 & $* *$ \\
\hline $\mathrm{C}_{4} \mathrm{H}_{10} \mathrm{Si}$ (1-methyl-silacyclobutane) & 76.38 & 77.23 & 76.50 & 77.18 & 77.02 & $* *$ \\
\hline $\mathrm{Cl}_{3} \mathrm{SiCH}_{3}$ & 25.44 & 26.34 & 24.28 & 25.83 & 22.20 & ${ }^{* *}$ \\
\hline $\mathrm{Cl}_{2} \mathrm{Si}\left(\mathrm{CH}_{3}\right)_{2}$ & 46.36 & 47.69 & 45.17 & 47.17 & 45.22 & ** \\
\hline$\left(\mathrm{CH}_{3}\right)_{3} \mathrm{SiCN}$ & 71.85 & 72.45 & 70.99 & 72.59 & 73.95 & ** \\
\hline $\mathrm{SiH}_{3} \mathrm{CN}$ & 19.27 & 20.49 & 19.36 & 19.98 & 19.59 & $* *$ \\
\hline $\mathrm{Si}(\mathrm{OH})_{4}$ & 34.56 & 32.43 & 32.10 & 34.64 & 32.38 & $* *$ \\
\hline $\mathrm{HSi}(\mathrm{OH})_{3}$ & 30.79 & 29.60 & 28.85 & 30.88 & 28.98 & $* *$ \\
\hline
\end{tabular}


Vibrational Zero-Point Energy of Organosilicon Compounds

DOI: $h t t p: / / d x$. doi.org/10.5772/intechopen.87021

\begin{tabular}{|c|c|c|c|c|c|c|}
\hline \multirow[t]{2}{*}{ Compound } & \multicolumn{5}{|c|}{$\mathrm{ZPE}(\mathrm{kcal} / \mathrm{mol})$} & \multirow[b]{2}{*}{ Ref. } \\
\hline & Exp. & Eq. $(5)^{\mathbf{a}}$ & $\mathrm{AM}^{\mathrm{b}}$ & $\begin{array}{l}\text { B3LYP/ } \\
6-31 G^{*} \mathrm{c}\end{array}$ & Eq. $(4)^{d}$ & \\
\hline $\mathrm{H}_{3} \mathrm{SiOSiH}_{3}$ & 32.90 & 35.38 & 30.93 & 33.16 & 32.91 & $* *$ \\
\hline $\mathrm{Si}(\mathrm{OH})_{3}-\mathrm{O}-\mathrm{Si}(\mathrm{OH})_{3}$ & 55.86 & 52.36 & 51.11 & 56.39 & 53.31 & ** \\
\hline $\mathrm{C}_{2} \mathrm{Si}_{2} \mathrm{H}_{8} \mathrm{O}$ (1-oxa-2,5-disilacyclopentane) & 56.90 & 56.93 & 55.41 & 57.43 & 54.91 & $* *$ \\
\hline$\left(\mathrm{H}_{2} \mathrm{SiO}\right)_{3}$ (cyclotrisiloxane) & 41.40 & 40.72 & 38.12 & 41.43 & 36.20 & ** \\
\hline$\left(\mathrm{H}_{2} \mathrm{SiO}\right)_{4}$ (cyclotetrasiloxane) & 54.91 & 54.99 & 51.19 & 55.05 & 50.33 & $* *$ \\
\hline$\left(\mathrm{C}_{2} \mathrm{H}_{5} \mathrm{O}\right)_{4} \mathrm{Si}$ & 171.18 & 174.09 & 170.16 & 172.95 & 177.34 & ${ }^{* *}$ \\
\hline $\mathrm{CH}_{3} \mathrm{SiH}_{2} \mathrm{SiH}_{2} \mathrm{CH}_{3}$ & 63.83 & 65.90 & 62.65 & 65.31 & 65.75 & $* *$ \\
\hline $\mathrm{H}_{3} \mathrm{SiSiH}_{2} \mathrm{SiH}_{3}$ & 39.94 & 41.41 & 36.92 & 40.19 & 40.24 & $* *$ \\
\hline$\left(\mathrm{Me}_{3} \mathrm{Si}\right)_{2} \mathrm{SiH}_{2}$ & 143.61 & 145.34 & 141.84 & 145.47 & 148.96 & $* *$ \\
\hline$\left(\left(\mathrm{CH}_{3}\right)_{3} \mathrm{Si}\right)_{3} \mathrm{SiH}$ & 205.33 & 207.45 & 204.16 & 208.86 & 214.05 & $* *$ \\
\hline $\mathrm{CH}_{3} \mathrm{OSi}\left(\mathrm{CH}_{3}\right)_{3}$ & 91.48 & 94.07 & 90.79 & 92.94 & 94.66 & $* *$ \\
\hline $\mathrm{Et}_{3} \mathrm{SiOH}$ & 126.18 & 127.66 & 125.19 & 128.10 & 130.90 & $* *$ \\
\hline $\mathrm{Ph}_{3} \mathrm{SiOH}$ & 172.20 & $176.01^{\mathrm{e}}$ & 174.18 & 173.66 & 177.46 & $* *$ \\
\hline nbutyl $_{3} \mathrm{SiOH}$ & 228.34 & 231.16 & 228.10 & 231.39 & 239.62 & $* *$ \\
\hline$\left(\mathrm{CH}_{3}\right)_{3} \mathrm{SiOSi}\left(\mathrm{CH}_{3}\right)_{3}$ & 134.88 & 139.31 & 133.95 & 137.52 & 141.63 & $* *$ \\
\hline$\left(\mathrm{CH}_{3}\right)_{3} \mathrm{SiOCOCH}_{3}$ & 97.34 & 97.38 & 97.00 & 98.80 & 101.94 & $* *$ \\
\hline $\begin{array}{l}\mathrm{C}_{8} \mathrm{H}_{16} \mathrm{SiO}_{2} \text { (4-trimethylsiloxy-3-penten-2- } \\
\text { one) }\end{array}$ & 134.19 & 137.23 & 134.29 & 135.98 & 142.06 & $* *$ \\
\hline $\begin{array}{l}\left(\mathrm{CH}_{3}\right)_{3} \mathrm{SiOCOCF}_{3} \\
\text { (trimethylsilyltrifluoroacetate) }\end{array}$ & 84.22 & 85.17 & 84.27 & 84.87 & 90.39 & ${ }^{* *}$ \\
\hline $\mathrm{C}_{3} \mathrm{H}_{7} \mathrm{SiCl}$ (cis-cyclopropylchlorosilane) & 55.47 & 55.80 & 53.87 & 55.03 & 54.00 & [79] \\
\hline \multicolumn{7}{|c|}{$\begin{array}{l}{ }^{a} \text { Values determined by our model. } \\
{ }^{b} \text { Values scaled by } 0.95[80] . \\
\text { cValues scaled by } 0.96[80] . \\
{ }^{d} \text { Values computed by Schulman-Disch extended empirical formula. } \\
\text { eValues adjusted by Eq. }(6) . \\
{ }^{* *} \text { Values calculated with HF/6-31G }{ }^{*} \text { and scaled by } 0.89 \text { [80]. }\end{array}$} \\
\hline
\end{tabular}

Table 2.

Comparison of computed (empirical Eq. (5), AM1, B3LYP/6-31 $G^{*}$, empirical Eq. (4)) zero-point energy with experimental values.

energies for the same organosilicon compounds using semiempirical (AM1) and DFT(B3LYP/6-31G ${ }^{*}$ ) methods. Results are summarized in Table 2. To correct calculated ZPEs, the scaling factors of Scott and Radom [80] are used.

Based on these results, the values calculated at the DFT(B3LYP/6-31G ${ }^{*}$ level are closer to the experimental data than those obtained at the semiempirical (AM1) level. The mean error is 2.50 (5.45\%) for AM1, compared to 1.08 (1.86\%) for DFT $\left(\mathrm{B} 3 \mathrm{LYP} / 6-31 \mathrm{G}^{*}\right)$. The correlations obtained between the experimental values and those calculated by using AM1 and DFT methods are represented, respectively, in Figure 4a and $\mathbf{b}$. Examination of this correlations shows that these methods are able to calculate accurately the zero-point vibration energies of the organosilicon compounds with a small advantage for the DFT method. For the adjustment of calculated ZPEs, the use of the regression line of the form $\mathrm{ZPE}_{\exp }=\mathrm{aZPE}$ theor $($ Table 3) permits to reduce the mean error of $2.5-1.83 \mathrm{kcal} / \mathrm{mol}$ for AM1 and from 1.08 to $1.06 \mathrm{kcal} / \mathrm{mol}$ for DFT(B3LYP/6-31G ${ }^{*}$ ). When the intercept is different from zero, 


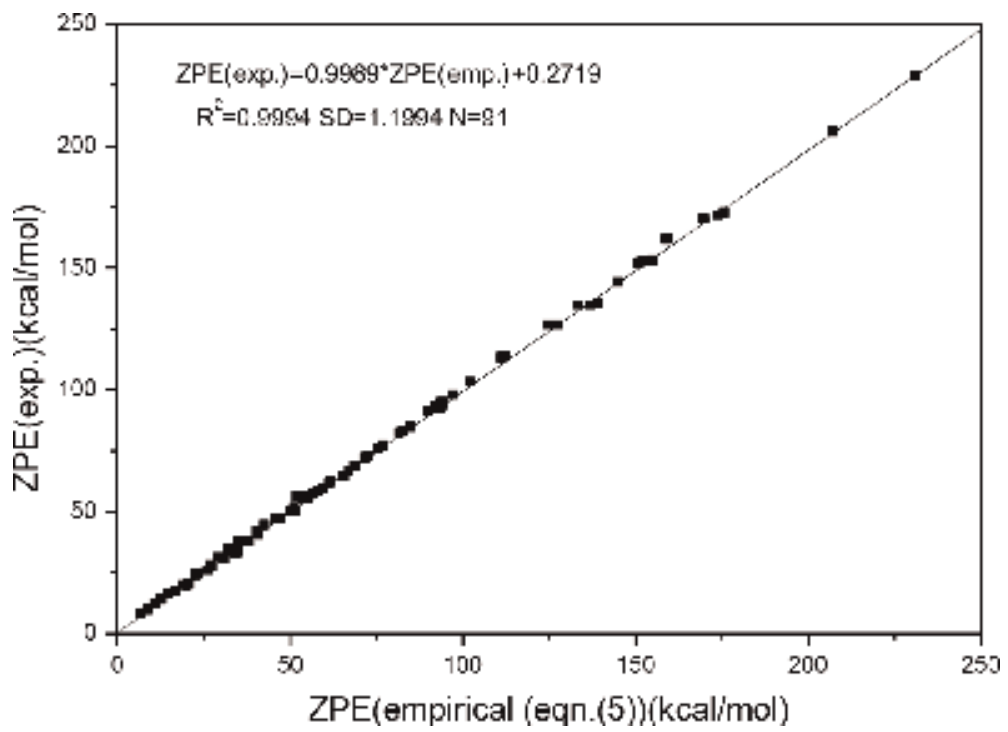

Figure 2.

Correlation between experimental ZPEs and empirical values calculated using Eq. (5).

\begin{tabular}{|c|c|c|c|c|c|}
\hline \multirow[t]{3}{*}{ Calculation method } & \multicolumn{5}{|c|}{ Linear model } \\
\hline & \multicolumn{2}{|c|}{$\mathrm{ZPE}_{\text {exp }}=\mathrm{aZPE_{ \text {theor } }}$} & \multicolumn{3}{|c|}{$\mathrm{ZPE}_{\exp }=\mathbf{b}+\mathbf{a Z P E _ { \text { theor } }}$} \\
\hline & $\mathbf{a}$ & $\mathbf{R}^{2}$ & $\mathbf{a}$ & $\mathbf{b}$ & $\mathbf{R}^{2}$ \\
\hline Proposed empirical model (Eq. (5)) & $0.993 \pm 0.002$ & 0.9998 & 0.99 & 0.272 & 0.9994 \\
\hline AM1 & $1.025 \pm 0.003$ & 0.9992 & 0.95 & 2.212 & 0.9982 \\
\hline DFT (B3LYP/6-31G*) & $0.999 \pm 0.002$ & 0.9996 & 0.95 & 0.304 & 0.9986 \\
\hline Schulman-Disch extended model (Eq. (4)) & $0.984 \pm 0.0038$ & 0.9986 & 0.95 & 3.545 & 0.9972 \\
\hline
\end{tabular}

Table 3.

Coefficients $a, b$, and $R^{2}$ in equations $Z P E_{\text {exp }}=b+a Z P E_{\text {theor }}$ in both cases $b=0$ and $b \neq 0$.

\begin{tabular}{lccccc}
\hline Atom & Increment & Ref. & Atom & Increment & Ref. \\
\hline $\mathrm{H}$ & 7120 & {$[25]$} & $\mathrm{Cl}$ & 2220 & {$[26]$} \\
\hline $\mathrm{C}$ & 3880 & {$[25]$} & $\mathrm{S}$ & 1870 & {$[27]$} \\
\hline $\mathrm{N}$ & 4050 & {$[26]$} & $\mathrm{Br}$ & 1600 & {$[27]$} \\
\hline $\mathrm{O}$ & 3400 & {$[26]$} & $\mathrm{Si}$ & -3510 & {$[28]$} \\
\hline $\mathrm{F}$ & 3270 & {$[26]$} & $\mathrm{P}$ & 0.035 & {$[29]$} \\
\hline
\end{tabular}

Table 4 .

Atom contributions to ZPE (in $\mathrm{kcal} / \mathrm{mol}$ ).

the average error decreases from 2.5 to $1.37 \mathrm{kcal} / \mathrm{mol}$ for AM1 and from 1.08 to 1.00 for DFT(B3LYP/6-31G ${ }^{*}$ ).

As a result, the four estimates of vibrational zero-point energy of the organosilicon compounds (our empirical model, Schulman-Disch extended empirical formula, AM1, and DFT) are correct. But the empirical approaches have the advantage of simplicity and speed. In addition, the approach based on bond contributions additivity has also the advantage of providing different values of ZPE for 


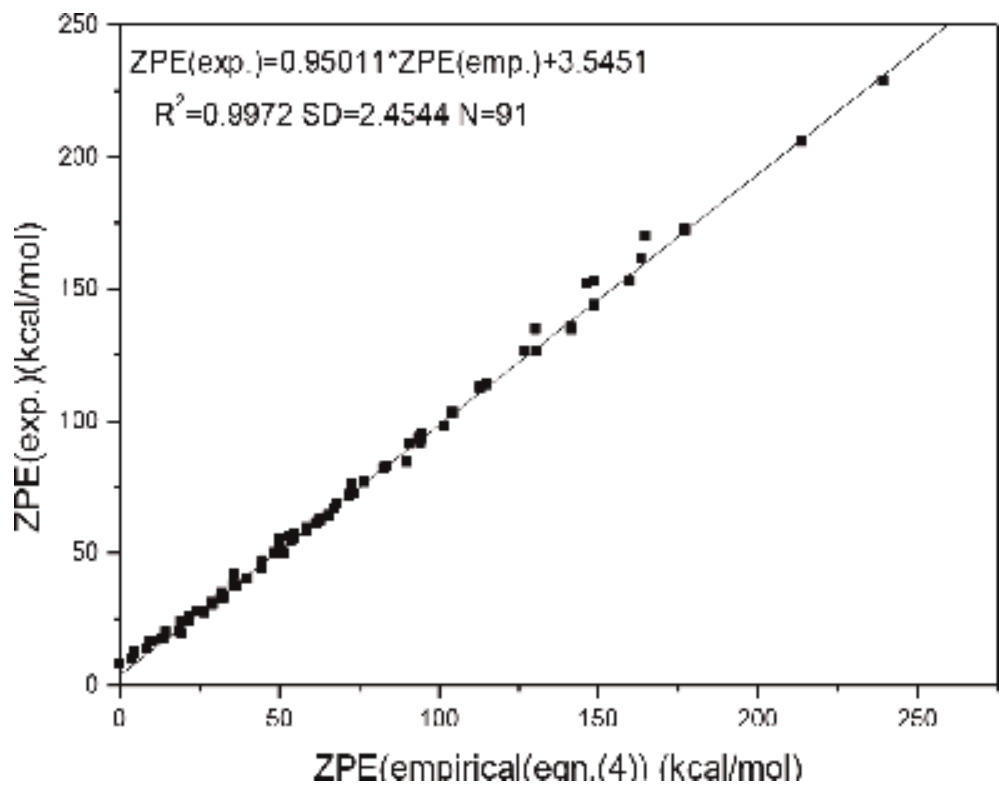

Figure 3 .

Correlation between experimental ZPEs and empirical values calculated using Eq. (4).
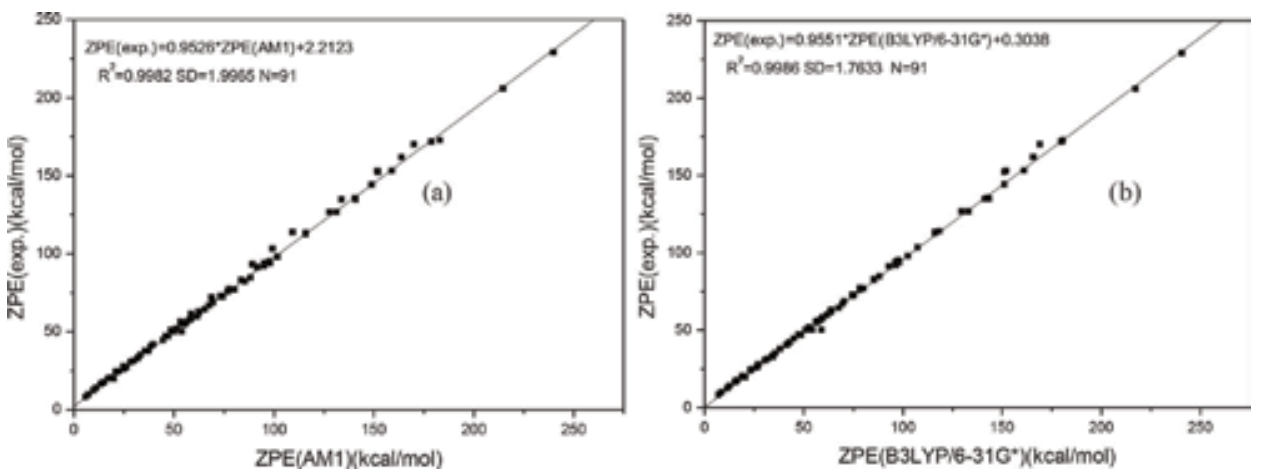

Figure 4.

(a) Correlation between experimental and theoretical (AM1) ZPE and (b) correlation between experimental and theoretical $\left(B_{3} L Y P / 6-31 G^{*}\right) Z P E$.

the function isomers. Furthermore, the adjustment of calculated values, empirically or using quantum methods, with the $\mathrm{ZPE}_{\exp }=\mathrm{b}+\mathrm{aZPE} \mathrm{E}_{\text {theor }}$ model makes the four estimates comparable.

\section{Conclusions and outlook}

In this chapter, we reported the extension of our empirical relationship established in 2011 for the computation of zero-point vibrational energies (ZPE) of organosilicon compounds. The bond contributions of $\mathrm{Si}-\mathrm{H}, \mathrm{Si}-\mathrm{C}, \mathrm{Si}-\mathrm{Cl}, \mathrm{Si}-\mathrm{O}$, and $\mathrm{Si}-\mathrm{Si}$ were determined. The application of the proposed empirical model to more than 90 organosilicon shows the reliability of this model. The results derived from this model are compared with those obtained by quantum chemistry methods (semiempirical (AM1) and DFT (B3LYP/6-31G $\left.{ }^{*}\right)$ ) on the one hand and to those obtained by similar empirical approach on the other hand. As a result, the empirical 
model provides a simple, fast, and accurate way to estimate the vibrational zeropoint energies of organosilicon compounds.

Establishing these empirical rules is becoming increasingly important. Indeed, thermodynamic data play an important role in the understanding and design of chemical processes. Experimental methods require appropriate equipment, sufficient product purity, time, and cost of experience. The use of such techniques becomes difficult for toxic compounds. In addition, the large difference between the number of synthesized compounds and the available experimental data continues to increase. In such situation, the practical approach is to use predictive models to estimate the properties of compounds from the molecular structures. In this outlook, we propose to extend the field of application of the proposed empirical model to other compounds such as organoborons, organomagnesians, etc., and to establish similar approaches to estimate other thermodynamic quantities such as enthalpy of formation $\left(\Delta \mathrm{H}_{\mathrm{f}}^{0}\right)$, entropy $\left(\mathrm{S}^{0}\right)$, and heat capacity $(\mathrm{C})$.

\section{List of abbreviations}

ZPE zero-point vibrational energy

AM1 Austin model 1 (developed by Michael Dewar and collaborators

$\mathrm{H}_{\mathrm{vib}}(\mathrm{T}) \quad$ vibrational enthalpy of the molecule

$\mathrm{S}_{\mathrm{vib}}(\mathrm{T}) \quad$ entropy due to vibrational motion

$\mathrm{H}(\mathrm{T})-\mathrm{H}(0) \quad$ thermal correction

HF/6-31G ${ }^{*} \quad$ Hartree-Fock theory in combination with the 6-31G(d) basis set

DFT

B3LYP/6-31G* density functional theory

Becke's three parameter exchange function (B3) with Lee-YangParr correlation function (LYP) functional coupled with 6-31G basis set

Eqn equation

\section{Author details}

Mahmoud Rahal ${ }^{1,2 *}$ and Abdeslam El Hajbi ${ }^{2}$

1 Centre Régional des Métiers d'Education et de Formation, Région Laayoune Sakia El Hamra, Laayoune, Morocco

2 Laboratoire de Chimie Physique, Faculté des Sciences, Université Chouaib Doukkali, El Jadida, Morocco

*Address all correspondence to: mrahal71@gmail.com

\section{IntechOpen}

(C) 2019 The Author(s). Licensee IntechOpen. This chapter is distributed under the terms of the Creative Commons Attribution License (http://creativecommons.org/licenses/ by/3.0), which permits unrestricted use, distribution, and reproduction in any medium, provided the original work is properly cited. (c) BY 


\section{References}

[1] Grice ME, Politzer P. Use of molecular stoichiometry to estimate vibrational-energy. Chemical Physics Letters. 1995;244:295-298

[2] Irikura KK. Experimental vibrational zero-point energies: Diatomic molecules. Journal of Physical and Chemical Reference Data. 2007;36: 389-397

[3] Pilcher G. In: Hartley FR, editor. The Chemistry of Organophosphorus Compounds, Vol. 1. New York: John Wiley \& Sons; 1990. p. 127

[4] Mollendal H, Konovalov A, Guillemin JC. Synthesis and microwave spectrum of (2-chloroethyl)phosphine $\left(\mathrm{ClCH}_{2} \mathrm{CH}_{2} \mathrm{PH}_{2}\right)$. The Journal of Physical Chemistry. A. 2009;113: 12904-12910

[5] Durham HD, Ecobichon DJ. An assessment of the neurotoxic potential of fenitrothion in the hen. Toxicology. 1986;41:319-332

[6] Ecobichon DJ, Davies JE, Doull J, Ehrich M, Joy R, McMillan D, et al. In: Baker SR, Wilkinson CF, editors. Neurotoxic Effects of Pesticides, in the Effects of Pesticides on Human Health, Vol. 18. Princeton, NJ: Princeton Scientific; 1990. p. 131

[7] Dorofeeva OV, Ryzhova ON, Zverev VG. Computational study of the thermodynamic properties of organophosphorus(V) compounds. Journal of Molecular Structure (THEOCHEM). 2007;811: 267-279

[8] Dorofeeva OV, Moiseeva NF. Computational study of the thermochemistry of organophosphorus (III) compounds. The Journal of Physical Chemistry. A. 2006;110: 8925-8932
[9] Sidell FR. Soman and sarin: Clinical manifestations and treatment of accidental poisoning by organophosphates. Clinical Toxicology. 1974;7:1-17

[10] Smeyers YG, Villa M. Influence of the vibrational zero-point energy correction on the amine inversion barrier and the far-infrared spectrum of methylamine. Chemical Physics Letters. 2000;324:273-278

[11] Sana M, Leroy G, Peeters D, Wilante C. The theoretical study of the heats of formation of organic compounds containing the substituents $\mathrm{CH}_{3}, \mathrm{CF}_{3}$, $\mathrm{NH}_{2}, \mathrm{NF}_{2}, \mathrm{NO}_{2}, \mathrm{OH}$ and $\mathrm{F}$. Journal of Molecular Structure (THEOCHEM). 1988;164:249-274

[12] Dykstra CE, Shuler K, Young RA, Bai Z. Anharmonicity effects on zero point energies of weakly bound molecular clusters. Journal of Molecular Structure (THEOCHEM). 2002;591: 11-18

[13] Fliszar S, Poliquin F, Badilescu I, Vauthier E. Structure dependent regularities of zero-point plus heat content energies in organic molecules. Canadian Journal of Chemistry. 1988; 66:300-303

[14] Tajti A, Szalay PG, Császár AG, Kállay M, Gauss J, Valeev EF, et al. HEAT: High accuracy extrapolated ab initio thermochemistry. The Journal of Chemical Physics. 2004;121:11599-11613

[15] Zahedi-Tabrizi M, Tayyari F, Moosavi-Tekyeh Z, Jalali A, Tayyari SF. Structure and vibrational assignment of the enol form of 1,1,1-trifluoro-2,4pentanedione. Spectrochimica Acta A. 2006;65:387-396

[16] Merrick JP, Moran D, Radom L. An evaluation of harmonic vibrational frequency scale factors. The Journal of 
Physical Chemistry. A. 2007;111: 11683-11700

[17] Oi T, Popowics A, Ischida T. Additivity of vibrational zero-point energy. The Journal of Physical Chemistry. 1986;90:3080-3088

[18] Oi T, Ischida T. Correlation of zeropoint energy with molecular structure and molecular forces. Development of the approximation. The Journal of Physical Chemistry. 1983;87:1067-1073

[19] Oi T, Ischida T. Correlation of zeropoint energy with molecular structure and molecular forces. III:

Approximation for H/D isotope shifts and linear frequency sum rule. The Journal of Physical Chemistry. 1984;88: 2057-2061

[20] Flanigan MC, Kormornicki A, McIver JW Jr. In: Segal GA, editor. Electronic Structure Calculation. New York: Plenum Press; 1977

[21] Császár AG, Furtenbacher T. Zerocost estimation of zero-point energies. The Journal of Physical Chemistry. A. 2015;119:10229-10240

[22] Barić B, Maksić ZB, Vianello R. On the atomic additivity of zero point vibrational energy in molecules. Journal of Molecular Structure (THEOCHEM). 2004;672:201-207

[23] Arimoto S. Preliminary considerations towards a theoretical foundation of the zero energy additivity rules. Physics Letters A. 1985;113A: 126-132

[24] Bernstein HJ. The physical properties of molecules in relation to their structure. I. Relations between additive molecular properties in several homologous series. The Journal of Chemical Physics. 1952;20:263-269

[25] Schulman JM, Disch RL. A simple formula for the zero-point energies of hydrocarbons. Chemical Physics Letters. 1985;113:291-293

[26] Ibrahim MR, Fataftah ZA.

Estimation of zero-point energies of compounds containing $\mathrm{N}, \mathrm{O}, \mathrm{Cl}$ and $\mathrm{F}$ atoms. Chemical Physics Letters. 1986; 125:149-154

[27] Ibrahim MR, Fataftah ZA.

Estimation of zero-point energies of bromo and thio compounds. Chemical Physics Letters. 1987;136:583-587

[28] AbdulHussain NS, Fleifel IA. Vibrational zero-point energies of nonaromatic silicon compounds. Journal of Basrah Researches. 2012;38:106-113

[29] Rahal M, Bouabdallah I, Malek F, El Hajbi A. A simple way to predict vibrational zero point energy of organophosphorus(III) compounds. Computational and Theoretical Chemistry. 2015;1068:13-20

[30] Ruzsinszky A, Kristyan S, Margitfalvi JL, Csonka GI. Rapid estimation of zero-point energies of molecules using Hartree-Fock atomic partial charges. The Journal of Physical Chemistry. A. 2003;107:1833-1839

[31] Pitzer KS. Thermodynamics of gaseous hydrocarbons: Ethane, propane, propylene, n-butane, isobutane, 1 butene, cis and trans 2-butenes, isobutene, and neopentane (tetramethylmethane). The Journal of Chemical Physics. 1937;5:473-479

[32] Cottrell TL. Binding energies in hydrocarbons. Journal of the Chemical Society. 1948;0:1448-1453

[33] Pitzer KS, Catalano E. Electronic correlation in molecules. III. The paraffin hydrocarbons. Journal of the American Chemical Society. 1956;78: 4844-4846

[34] Bernstein HJ, Pullin ADE. A sum rule for the vibrational frequencies of 
homologs. The Journal of Chemical Physics. 1953;21:2188-2195

[35] Bernstein HJ. Constitutive nature of the heat capacity of gases. The Journal of Chemical Physics. 1956;24:911-912

[36] Bernstein HJ. Bond and interaction contributions for calculating the heat of formation, diamagnetic susceptibility, molar refraction and volume, and thermodynamic properties of some substituted methanes. The Journal of Physical Chemistry. 1965;69: 1550-1564

[37] Shingu H, Fujimoto T. Empirical correlation between molecular structure and zero-point energies in hydrocarbons. The Journal of Chemical Physics. 1959;31:556-557

[38] Rahal M, Hilali M, El Hamadi A, El Mouhtadi M, El Hajbi A. Calculation of vibrational zero-point energy. Journal of Molecular Structure (THEOCHEM). 2001;572:73-80

[39] Rahal M, El Hajbi A. Vibrational zero-point energies of bromo compounds. Journal of Molecular Structure (THEOCHEM). 2004;668: 197-200

[40] Rahal M, Bouabdallah I, El Bouzaidi RD, Malek F, El Hajbi A. Vibrational zero point energy of organophosphorus (V) compounds. Vibrational Spectroscopy. 2016;86:173-180

[41] Dewar MJS, Zoebisch EG, Healy EF, Stewart JJP. Development and use of quantum mechanical molecular models. 76. AM1: A new general purpose quantum mechanical molecular model. Journal of the American Chemical Society. 1985;107:3902-3909

[42] Parr RG, Wang W. DensityFunctional Theory of Atoms and Molecules. Oxford University Press: New York; 1994
[43] Neumann R, Nobes RH, Handy NC. Exchange functionals and potentials. Molecular Physics. 1996;87:1-36

[44] Becke AD. Density-functional exchange-energy approximation with correct asymptotic behavior. Physics Review. 1988;A38:3098-30100

[45] Becke AD. Density-functional thermochemistry. III. The role of exact exchange. The Journal of Chemical Physics. 1993;98:5648-5652

[46] Lee C, Yang W, Paar RG. Development of the Colle-Salvetti correlation energy formula into a functional of the electron density. Physics Review. 1988;B37:785-786

[47] Frisch MJ, Trucks GW, Schlegel HB, Scuseria GE, Robb MA, Cheeseman JR, et al. Gaussian 03, Revision C.02.

Pittsburgh, PA: Gaussian Inc.; 2003

[48] Rahal M, El Mouhalhal N, El Hajbi

A. Détermination des grandeurs thermodynamiques par les méthodes semi-empiriques (AM1, PM3 ET MNDO). Annales de Chimie Science des Materiaux. 2006;31:103-112

[49] Rahal M, Bouabdallah I, El Hajbi A. Rapid estimation of vibrational zeropoint energies of silicon compounds. Computational and Theoretical Chemistry. 2013;1017:182-187

[50] Smith JG. The millimetre wave spectrum of trichlorosilane. Journal of Molecular Spectroscopy. 1986;120:

110-117

[51] Zhang Q, Wang S, Gu Y. Direct ab initio dynamics studies of the reactions of $\mathrm{H}$ with $\mathrm{SiH}_{4-\mathrm{n}} \mathrm{Cl}_{\mathrm{n}}(\mathrm{n}=1-3)$. The Journal of Physical Chemistry. A. 2002; 106:3796-3803

[52] Keister JW, Tomperi P, Baer T. Thermochemistry of gaseous ethylsilane cations. Journal of the American Society for Mass Spectrometry. 1998;9:597-605 
[53] Gamil GA, Panikar SS, El Defrawy AM, Kalasinsky VF, Durig JR.

Vibrational spectra, $r_{0}$ structural parameters, barriers to internal rotation, and $<\mathrm{i}>$ ab initio calculations of $\mathrm{ClCH}_{2} \mathrm{SiH}_{3}$, $\mathrm{Cl}_{2} \mathrm{CHSiH}_{3}, \mathrm{ClCH}_{2} \mathrm{SiF}_{3}$ and $\mathrm{Cl}_{2} \mathrm{CHSiF}_{3}$. Journal of Molecular Structure. 2009; 922:93-102

[54] Shimanouchi T. Tables of Molecular Vibrational Frequencies, Consolidated volume 1. Washington, DC: National Bureau of Standards; 1972. NSRDS NBS-39

[55] Khaikin LS, Grikina OE, Sipachev VA, Traetteberg M. The structure and spectra of 1-silylpropyne: Scaled quantum-chemical forcefields and vibrational effects. Journal of Molecular Structure. 2001;567:85-99

[56] Aleksa V, Klaeboe P, Nielsen CJ, Tanevska V, Guirgis GA. The conformations of bromomethyl dimethyl silane and bromomethyl dimethyl silane-d1 as studied by vibrational spectroscopy and by ab initio calculations. Vibrational Spectroscopy. 1998;17:1-30

[57] Bethke GW, Wilson MK. Vibrational spectrum of disilane. The Journal of Chemical Physics. 1957;26: 1107-1117

[58] Shainyan BA, Kleinpeter E. Conformational preferences of $\mathrm{Si}-\mathrm{Ph}, \mathrm{H}$ and $\mathrm{Si}-\mathrm{Ph}, \mathrm{Me}$ silacyclohexanes and 1,3thiasilacyclohexanes. Additivity of conformational energies in 1,1disubstituted heterocyclohexanes. Tetrahedron. 2012;68:114-125

[59] Swihart MT, Carr RW.

Thermochemistry and thermal decomposition of the chlorinated disilanes $\left(\mathrm{Si}_{2} \mathrm{H}_{\mathrm{n}} \mathrm{Cl}_{6-\mathrm{n}}, \mathrm{n}=0-6\right)$ studied by $\mathrm{ab}$ initio molecular orbital methods. The Journal of Physical Chemistry. A. 1997; 101:7434-7445
[60] Ye CC, Xie LF, Ju XH. Theoretical study of on thermodynamic properties and stabilities of n-silanes. Phosphorus, Sulfur and Silicon and the Related Elements. 2011;186:1948-1961

[61] Durig JR, Nashed YE, Qtaitat MA, Guirgis GA. Spectra and structure of silicon containing compounds. XXVI. Raman and infrared spectra, ro structural parameters, conformational stability, vibrational assignment, and ab initio calculations of vinyl silyl chloride. Journal of Molecular Structure (THEOCHEM). 2000;525:191-208

[62] Pana C, Guirgis GA, Durig JR. Infrared and raman spectra, conformational analysis, ab initio calculations and vibrational assignment of 2-chloroethylsilyl chloride. Journal of Molecular Structure. 2005;742:199-213

[63] Mohamed TA, Guirgis GA, Nashed YE, Durig JR. Spectra and structure of silicon containing compounds. Vibrational Spectroscopy. 2002;30: 111-120

[64] Guirgis GA, Nashed YE, Durig JR. Raman and infrared spectra, conformational stability, ab initio calculations and vibrational assignments for 2-chloroethyl silane. Journal of Molecular Structure. 1999;510:13-34

[65] Mohamed TA, Guirgis GA, Nashed YE, Durig JR. Spectra and structure of silicon-containing compounds. XXV. Raman and infrared spectra, $\mathrm{r} 0$ structural parameters, vibrational assignment, and $\mathrm{ab}$ initio calculations of ethyl chlorosilane-Si-d2. Structural Chemistry. 1999;10:333-348

[66] Lü R, Qiu G, Liu C. Theoretical study of ethanethiol adsorption on HZSM-5 zeolite. Journal of Natural Gas Chemistry. 2005;14:119-124

[67] Abdulaziz AA, Jaan L. Structure, vibrational spectra, and DFT and ab 
initio calculations of silacyclobutanes. Organometallics. 2008;27:3435-3443

[68] Mohamed TA, Guirgis GA, Nashed YE, Durig JR. Spectra and structures of silicon-containing compounds. XXIV.

Raman and infrared spectra, $\mathrm{r}_{0}$ structural parameters, vibrational assignment, barriers to internal rotation, and $\mathrm{ab}$ initio calculations of ethylsilane. Structural Chemistry. 1998;9:255-264

[69] Montejo M, Ureña FP, Márquez F, González JJL. The vibrational spectra of $\left(\mathrm{CH}_{3}\right)_{3} \mathrm{SiX}(\mathrm{S}=\mathrm{H}, \mathrm{F}, \mathrm{Br})$ molecules, revisited transferable scale factor sets for methylsilane derivatives.

Spectrochimica Acta Part A. 2005;62: 1058-1069

[70] Durig JR, Pana C, Guirgis GA. Spectra and structure of silicon containing compounds. XXXII. Raman and infrared spectra, conformational stability, vibrational assignment and ab initio calculations of n-propylsilane-d0 and Si-d3. Spectrochimica Acta Part A. 2003;59:979-1002

[71] Duriga JR, Pana C, Guirgis GA. Spectra and structure of silicon containing compounds. XLII. Conformational stability, ab initio calculations, $r_{0}$ structural parameters, and vibrational assignments for ethylmethylsilane and ethylmethylsilane-Si-d2. Journal of Molecular Structure. 2004;88:95-109

[72] Durig JR, Panikar SS, Guirgis GA, Gounev TK, Ward RM, Peeblesc RA, et al. Conformational stability, $\mathrm{r}_{0}$ structural parameters, barriers to internal rotation, vibrational spectra and $\mathrm{ab}$ initio calculations of $\mathrm{c}-\mathrm{C}_{3} \mathrm{H}_{5} \mathrm{SiH}_{2} \mathrm{CH}_{3}$. Journal of Molecular Structure. 2009; 923:1-12

[73] Mohamed TA. Structural parameters, barriers to internal rotation, normal coordinate analysis and quantum mechanics calculations of 1,1,1-trimethyldisilane. Journal of Molecular Structure (THEOCHEM). 2003;635:161-172

[74] Durig JR, Zhao W, Zhu X, Geyer TJ, Dakkouri M. Spectra and structure of small ring compounds. LXV. Raman and infrared spectra, conformational stability, and vibrational assignment of cyclopentylsilane. Journal of Molecular Structure. 1995;351:31-49

[75] Zheng C, Subramaniam S, Kalasinsky VF, Durig JR. Raman and infrared studies supported by ab initio calculations for the determination of conformational stability, silyl rotational barrier and structural parameters of cyclohexyl silane. Journal of Molecular Structure. 2006;785: 143-159

[76] Guirgis GA, Nashed YE, Gounev TK, Durig JR. Conformational stability, structural parameters, vibrational frequencies, and Raman and infrared intensities of allylsilane. Structural Chemistry. 1998;9:265-277

[77] Guirgis GA, Zhengb C, Nashed YE, Mohamed TA, Durig JR. Spectra and structure of silicon containing compounds XXXV infrared and Raman spectra, vibrational assignment, conformational stability and ab initio calculations of dichloromethyldimethyl silane. Journal of Molecular Structure. 2003;649:7-24

[78] Durig JR, Hur SW, Guirgis GA. Infrared and Raman spectra, conformational stability, vibrational assignment and ab initio calculations of methylvinyl silyl chloride.

Spectrochimica Acta Part A. 2003;59: 2449-2471

[79] Kalincsák F, Pongor G. Extension of the density functional derived scaled quantum mechanical force field procedure. Spectrochimica Acta Part A. 2002;58:999-1011 
[80] Scott AP, Radom L. Harmonic

vibrational frequencies: An evaluation

of Hartree-Fock, Moller-Plesset,

quadratic configuration interaction,

density functional theory, and

semiempirical scale factors. The Journal

of Physical Chemistry. 1996;100:

16502-16513 


\title{
Analysis of the Absorption Phenomenon through the Use of Finite Element Method
}

\author{
Minerva Robles-Agudo, Martha Patricia Gonzalez-Tejeda, \\ Porfirio Esau Martinez-Muñoz and Ignacio Rojas-Rodríguez
}

\begin{abstract}
In this text, a numerical model using the finite element method (FEM) is developed to describe absorption phenomena using the Beer-Lambert law. Numerical results are compared against experimental measurements made on alpha brass, characterized by photothermal radiometry (PTR). Results from the numerical analysis are in good agreement with the measurements obtained from the radiometry tests. In this technique the material is subjected to a laser heating, producing a thermal wave that is captured with a detector by means of the amplitude and phase parameters. The analysis of the data obtained from six samples provides information about their thermal properties, such as conductivity and diffusivity, which can be correlated with structural changes in a material. Results from this research lead to the characterization of mechanical properties of metallic materials.
\end{abstract}

Keywords: light absorption, Beer-Lambert law, photothermal radiometry, finite element method

\section{Introduction}

Applications of materials in the industry depend on their physical-chemical properties. Metal and semiconductor materials have had a big relevance for humanity development, facilitating the life and making possible technological advances still today. The properties and characteristics of metals are taken into account for the selection of materials according to the function we will perform.

Copper alloys are materials known for a wide range of applications. Currently more than 400 copper alloys are known, among which brass and bronze are distinguished. It is possible to vary the amounts of copper and zinc in these alloys to obtain a variety of brass with different properties [1,2].

Brass is considered one of the most important copper alloys due to the amount of zinc that can be varied considerably, ranging between 5 and $45 \%$ by weight. It is possible to produce a wide variety of brass alloys with different technological properties for various commercial and industrial applications. Among the most commonly used brass in engineering areas is high-strength brass, suitable for heavy loads, with adequate properties to resist wear and corrosion. Among the main advantages of these materials are their mechanical properties by thermal treatment and their low cost [3-5]. 
In 2010 Ozgowiez et al. [6] analyzed the influence of recrystallization annealing temperature on the microstructure and mechanical properties of $\mathrm{Cu} 30 \% \mathrm{Zn}$ brass, which was subjected to cold deformation with a variable tension in the rolling process. The mechanical test showed that the properties of the brass deteriorated and the properties of the plastic increased as the recrystallization temperature increased within the range of $400-650^{\circ} \mathrm{C}$ [7].

In 1967 Bailey studied the structure and strength of an alpha brass with $20 \%_{\mathrm{w}}$ $\mathrm{Zn}, 6 \%_{\mathrm{w}} \mathrm{Ni}$, and $1.5 \%_{\mathrm{w}} \mathrm{Al}$. Brass samples were subjected to solution heat treatment (SHT) at $800^{\circ} \mathrm{C}$ for $2 \mathrm{~h}$ followed by quenching with water. Subsequently, the samples were thermally treated for $2 \mathrm{~h}$ at $300,400,500,600$, and $700^{\circ} \mathrm{C}$. The best mechanical properties were obtained at $500^{\circ} \mathrm{C} \mathrm{PHT}$ temperature [8].

Infrared photothermal radiometry (PTR) is an optical technique used for the characterization of metallic materials such as brass, to determine the influence of the precipitation heat treatment (PHT) temperature on metallurgical microstructure, thermal properties, and microhardness. This technique is based in light absorption and consists of a laser beam incident on a sample, which will absorb the radiation and emit a thermal wave that is detected by an infrared sensor. The signals of thermal wave are translated as amplitude and phase parameters. Compared with other characterization techniques, this technique has no destructive properties and has the advantage of not having direct contact with the sample to be analyzed.

One of the most important aspects to consider in the PTR is the process of light absorption in the material to be analyzed. As trivial as this may sound, absorption very often turns out to be the most critical and cumbersome step in laser processing. An enormous amount of work has been dedicated to investigating laser absorption mechanisms under various circumstances, and a great deal can be learned from this work for the benefit of laser-material processing.

Absorption process can be thought of as secondary "source" of energy inside the material. While driven by the incident beam, it tends to develop its own dynamics and can behave in ways deviating from the laws or ordinary optics. It is this "secondary" source, rather than the beam emitted by the laser device, which determines what happens to the irradiated material [9].

Certain wavelengths of light can be selectively absorbed by a substance or a material according to its molecular structure. The absorption of light occurs when an incident photon promotes the transition of an electron from a state of lower to higher energy. Excited electrons eventually lose this gained energy, and by a spontaneous radiation process, they return to their initial state.

The radiation emitted by a molecule, or an atom, after it has absorbed energy to place itself in an excited state, is defined as luminescence, depending on the nature of the excited state. All spectrophotometric methods are based on two laws that combined which are known as Beer-Lambert law. This law states that the light absorbed by a semitransparent medium is independent of the intensity of incident light, and each successive layer of the medium absorbs an equal fraction of the light passing through it. This amount of light can be calculated by Eq. (1), where $I_{0}$ is the incident light intensity, $I$ is the transmitted light intensity, $l$ is the length through which the light passes in the spectrophotometer cell, and $k$ is the constant of the medium:

$$
\log \left(\frac{I_{0}}{I}\right)=k l
$$

In the absorption spectrometry, the comparison of incident light intensity before and after the interaction with a sample can be carried out nowadays, by means of different software. In the development of theoretical models, it is possible to include 
properties of the materials and parameters that describe the process system, such as the direction or directions of the incidence of light and wavelength, among others.

Finite element method (FEM) is one of the most practical methods for solving engineering problems. This general numerical method is used to approximate solutions of partial differential equations very complicated in the resolution of problems that involve a high degree of mathematical and physical-mathematical performance. FEM is applied to character geometries and multiphysical behavior, both of material properties and of problems where it is generally not possible to obtain any analytical solution directly with the use of mathematical expressions. Some of the areas that use the finite element method to solve problems are heat transfer, fluid flow, mass transport, distribution of electromagnetic fields, and structural analysis, among others.

FEM allows obtaining an approximate numerical solution on a body, structure, or domain (continuous medium). On this domain certain differential equations are defined in an integral form that characterizes the physical behavior of the problem, dividing it into a high number of non-subdomains intersecting each other called "finite elements." This set of finite elements forms a partition of the domain also called discretization. Within each element there are a series of representative points called nodes. Two nodes are adjacent if they belong to the same finite element; in addition, a node on the edge of a finite element can belong to several elements. The set of nodes considering their adjacency relationships is called "mesh."

Calculations are carried out on this mesh of points called nodes, which serve as the basis for the discretization of the domain in finite elements. The creation of the mesh is done in a stage prior to the calculations called pre/process and usually with special programs called mesh generators. According to these adjacency or connectivity relations, the value of a set of unknown variables defined in each node and called degrees of freedom is related. The set of relations between the values of a given variable between the nodes can be written as a system of linear (or linearized) equations. The matrix of the said system of equations is called the system's stiffness matrix. The number of equations of said system is proportional to the number of nodes.

The FEM is widely used due to its generality and the ease of introducing complex calculation domains (in two or three dimensions). In addition, the method is easily adaptable to problems of heat transmission, fluid mechanics to calculate fields of velocities and pressures (computational fluid mechanics, CFD), or electromagnetic field. Given the practical impossibility of finding the analytical solution to these problems, often in engineering practice, numerical methods and, in particular, finite elements become the only practical alternative to calculation. One important property of the method is convergence; if finite element partitions are considered successively finer, the calculated numerical solution converges rapidly toward the exact solution of the system of equations.

For all the above, the FEM is an excellent method to consider the development of theoretical models for the analysis of the phenomenon of the absorption of radiation in materials. In this chapter, an analysis of absorption process as result of laser-material interactions using Beer-Lambert law is shown. In Section 2.1 a model absorption using COMSOL Multiphysics with finite element method in alpha brass is described. In Section 3 experimental results of photothermal radiometry technique applied to alpha brass are shown.

\section{Analysis of laser-material interactions using Beer-Lambert law}

When a light beam incident upon a semitransparent material, part of the energy supplied by it will be absorbed by the material. This process can be described through Beer-Lambert law. If we consider monochromatic and collimated light, 
such as laser light, and we despise losses by reflection and dispersion in the material, considering a minimum refraction and scattering, the Beer-Lambert law can be written in differential form for the light intensity $\mathbf{I}$ as

$$
\frac{\partial \boldsymbol{I}}{\partial z}=\alpha(T) \boldsymbol{I}
$$

where $\mathrm{z}$ is the coordinate along the beam direction and $\alpha(T)$ is the temperaturedependent absorption coefficient of the material. Because the temperature can vary in space and time, we must also solve the next governing partial differential equation for temperature distribution within the material:

$$
\rho C_{p} \frac{\partial T}{\partial t}-\nabla \cdot(k \nabla \mathrm{T})=\mathrm{Q}=\alpha(T) \boldsymbol{I}
$$

where $Q$ is the heat source term equals the absorbed light. Eqs. (2) and (3) present a bidirectionally coupled multiphysics problem that is possible to solve within the core architecture of COMSOL Multiphysics program based in the finite element method [10].

\subsection{Beer-Lambert law deviations}

Beer-Lambert law states that the optical density is directly proportional to the concentration of the species they absorb. However, deviations from this law can occur due to instrumental and intrinsic causes [11-13]. Among the main deviations from Beer-Lambert law are the following:

\subsubsection{Light scattering}

There are two dispersion phenomena, one depends on the size of the solute particle or any suspended material. Biological samples are usually cloudy because macromolecules or other large aggregates scatter light. The optical densities resulting from the scattering of light are proportional to $1 / \lambda^{4}$ (Rayleigh scattering) and can therefore be recognized as an absorption background which increases rapidly with the decrease in wavelength [14]. The second type of dispersion is known as the Raman scattering. In this phenomenon, part of the excitation energy of light is abstracted by vibrational modes of the solvent molecules. In the case of water or hydroxyl solvents, the most dominant vibrations that absorb this energy are the $\mathrm{OH}$ groups, whose vibration energy is observed at a wavelength of $3300 \mathrm{~cm}^{-1}$. The Raman signal of the solvents will be observed at a wavelength that is $3300 \mathrm{~cm}^{-1}$ less in energy than the excitation wavelength. The wavelength of the Raman scattering $\left(\lambda_{\mathrm{RA}}\right)$ can be calculated as $\lambda_{\mathrm{RA}}{ }^{-1}=\lambda_{\mathrm{ex}}{ }^{-1}-0.00033$ [15].

\subsubsection{Fluorescence}

If the optical density of the sample is high and if the absorbing species are fluorescent, the emitted light can reach the detector. This process will result in derivations of Beer-Lambert law. The effect can be minimized by maintaining the distance of the detector from the sample and decreasing the efficiency with which the emission fluorescence is collected.

\subsection{Use of COMSOL multiphysics to model absorption in alpha brass through FEM}

COMSOL Multiphysics is a general-purpose simulation software for modeling designs, devices, and processes in all fields of engineering, manufacturing, and 
scientific research. The platform product can be used on its own or expanded with functionality from any combination of add-on modules for simulating electromagnetic fields, structural mechanics, acoustics, fluid flow, heat transfer, and chemical engineering. The add-on modules and LiveLink ${ }^{\mathrm{TM}}$ products connect seamlessly for a modeling workflow that remains the same regardless of what you are modeling.

Engineers and scientists use the COMSOL Multiphysics ${ }^{\circledR}$ software to simulate designs, devices, and processes in all fields of engineering, manufacturing, and scientific research. This simulation platform encompasses all of the steps in the modeling workflow-from defining geometries, material properties, and the physics that describe specific phenomena to solving and postprocessing models for producing accurate and trustworthy results [16].

In this section a simple model in COMSOL developed for the analysis of laser light absorption in alpha brass samples is described. The geometry of the problem is described using a concentric double cylinder, where the external cylinder represents the sample being heated, with a diameter of $40 \mathrm{~mm}$, while the diameter of the internal cylinder represents the diameter of the laser beam, with a diameter of $10 \mathrm{~mm}$, as shown in Figure 1. According to the geometry defined in the problem, a collimated beam with a constant intensity distribution is considered, in which the effects of divergence are neglected. In the general analysis, the domain is divided into two volumes, which are equivalent to the two cylinders. These volumes represent the same material, but only Beer-Lambert law is resolved in the internal domain (internal cylinder), which is the only region in which the material is heated by the laser beam. To implement Beer-Lambert law, it starts by adding the PDE interface with the dependent variables and unit settings.

The equation itself is implemented via the general form PDE interface. Aside from the source term, $f$, all terms within the equation are set to zero; thus, the equation being solved is $f=0$. The source term is set to Iz- $\left(50[1 / \mathrm{m}]^{*}(1+(\mathrm{T}-300[\mathrm{~K}]) / 40[\mathrm{~K}])\right)^{*} \mathrm{I}$, where the partial derivative of light intensity with respect to the $\mathrm{z}$-direction is Iz, and the absorption coefficient is $\left(50[1 / \mathrm{m}]^{*}(1+(\mathrm{T}-300[\mathrm{~K}]) / 40[\mathrm{~K}])\right)$, which introduces temperature dependency for illustrative purposes. This one line implements the BeerLambert law for a material with a temperature-dependent absorption coefficient, assuming that it will also solve for the temperature field, $\mathrm{T}$, in this model.

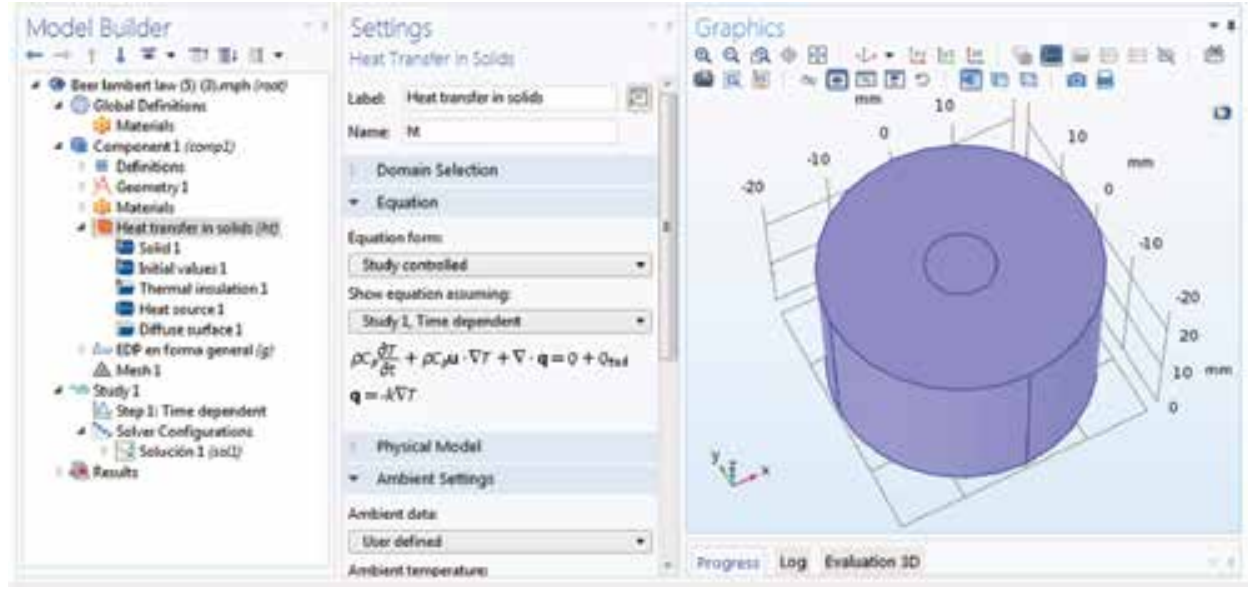

Figure 1.

Definition of the cylindrical geometry, parameters, and initial conditions for the absorption analysis model in alpha brass. 
Since this equation is linear and stationary, the initial values do not affect the solution for the intensity variable. The zero-flux boundary condition is the natural boundary condition and does not impose a constraint or loading term. It is appropriate on most faces, with the exception of the illuminated face. We will assume that the incident laser light intensity follows a Gaussian distribution with respect to distance from the origin. At the origin, and immediately above the material, the incident intensity is $0.3 \mathrm{~W} / \mathrm{mm}^{2}$. Some of the laser light will be reflected at the dielectric interface, so the intensity of light at the surface of the material is reduced to 0.95 of the incident intensity. This condition is implemented with a Dirichlet boundary condition. At the face opposite to the incident face, the default zero-flux boundary condition can be physically interpreted as meaning that any light reaching that boundary will leave the domain.

With these settings described above, the problem of temperature-dependent light absorption governed by the Beer-Lambert law has been implemented. It is, of course, also necessary to solve for the temperature variation in the material over time. In this case thermal conductivity of alpha brass of $93.32 \mathrm{~W} / \mathrm{m} / \mathrm{K}$ is considered, with a density of $2000 \mathrm{~kg} / \mathrm{m}^{3}$ and a specific heat of $1000 \mathrm{~J} / \mathrm{kg} / \mathrm{K}$ that is initially at $300 \mathrm{~K}$ with a volumetric heat source.

The heat source itself is simply the absorption coefficient times the intensity or, equivalently, the derivative of the intensity with respect to the propagation direction. Most other boundaries can be left at the default thermal insulation, which will also be appropriate for implementing the symmetry of the temperature field. However, at the illuminated boundary, the temperature will rise significantly, and radiative heat loss can occur. This can be modeled with the diffuse surface boundary condition, which takes the ambient temperature of the surroundings and the surface emissivity as inputs.

It is worth noting that using the diffuse surface boundary condition implies that the object radiates as a gray body. However, the gray body assumption would imply that this material is opaque. So how is it possible to use Beer-Lambert law, which is appropriate for semitransparent materials? It is possible to solve this apparent discrepancy by observing that the absorption capacity of the material is highly dependent on the wavelength. The depth of penetration is relatively large. However, when the part is heated, it is reradiated mainly in the long infrared regime. At long infrared wavelengths, it is possible to assume that the depth of penetration is very small and, therefore, the assumption that the volume of the material is opaque for radiation is valid.
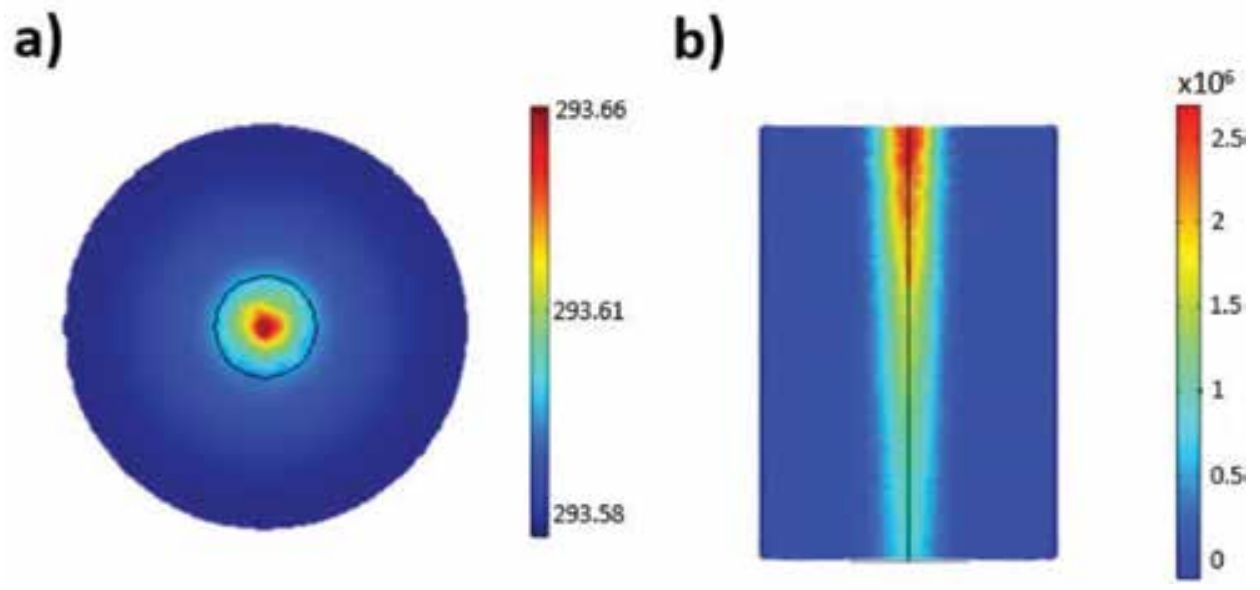

Figure 2.

Propagation of heat in the alpha brass material, (a) surface profile and (b) penetration profile. 


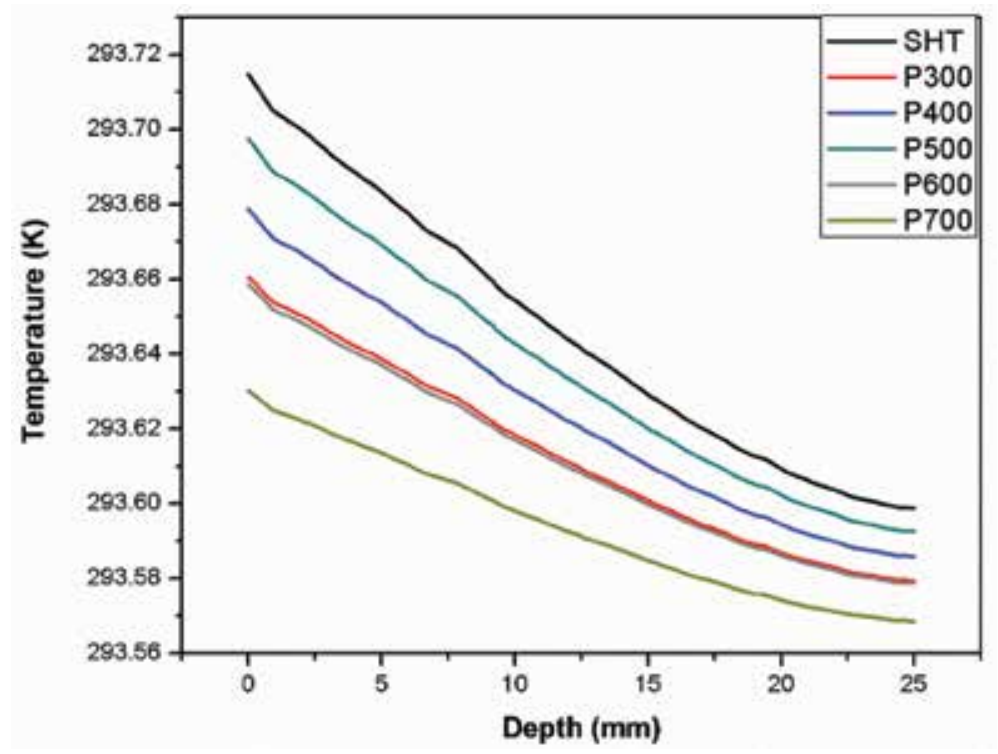

Figure 3.

Temperature as a function of depth along the centerline over 40 seconds of time, for six different samples of alpha brass.

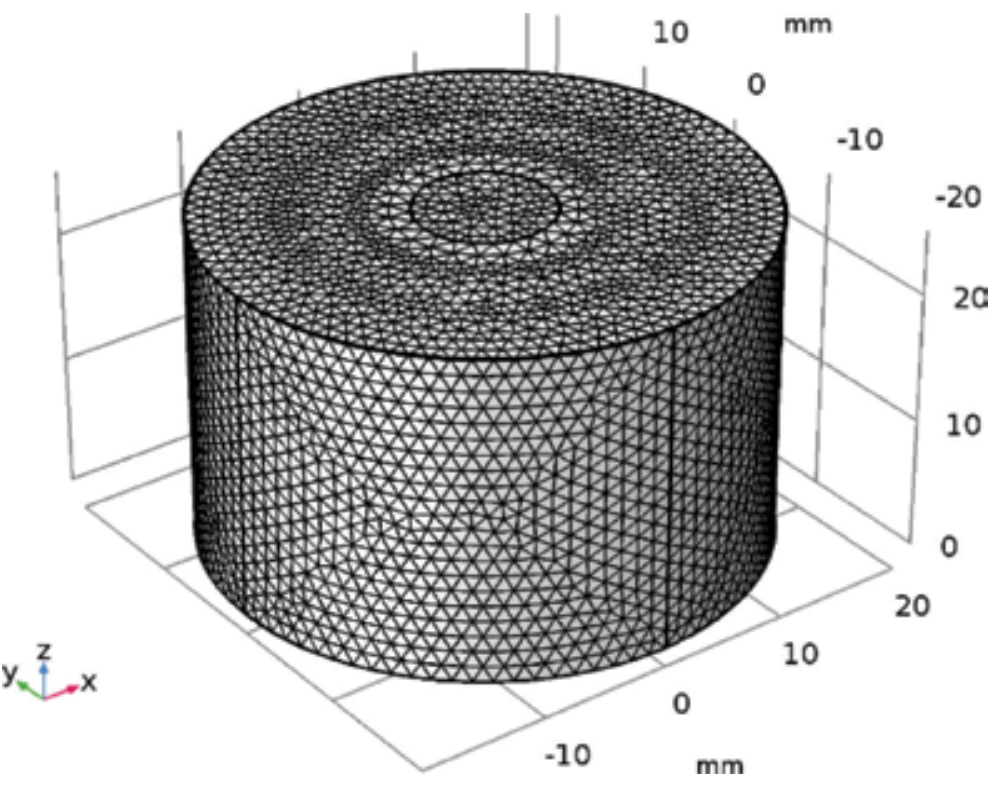

Figure 4 .

Creation of the mesh for the calculation of the heat distribution in alpha brass by means of the finite element method.

It is possible to solve this model either for the steady-state solution or for the transient response. In Figure 2 the propagation of heat in the alpha brass material is shown. In Figure 3 the changes of temperature as a function of depth along the centerline over a time of 40 seconds for six different kinds of alpha brass are shown.

In Figure 4 the mesh used to solve the equations through finite element is shown. Although it is not necessary to use a swept mesh in the absorption direction, applying this feature provides a smoot solution for the light intensity with relatively fewer elements than a tetrahedral mesh. The plot of temperature with respect to 
depth at the centerline illustrates the effect of the varying absorption coefficient due to its increase.

\section{Experimental methods to characterize Kunial alpha brass through thermal treatments}

The Kunial alpha brass is a copper-zinc alloy plus nickel and corresponds to an alpha brass with chemical composition of $20 \% \mathrm{Zn}_{\mathrm{w}}, 6 \% \mathrm{Ni}_{\mathrm{w}}, 1.5 \% \mathrm{Al}_{\mathrm{w}}$, and the balance $\mathrm{Cu}$. Six samples were characterized; one of them with solution heat treatment (SHT), as reference. Where SHT is the heating of an alloy to a temperature at which a particular constituent will enter into solid solution followed by cooling at a rate fast enough to prevent the dissolved constituent from precipitating. The other five samples were treated at different precipitation heat treatment (PHT) temperatures, as shown in Table 1. The thermal treatment by precipitation involves the addition of impurity particles to increase the strength of a material. PHT Samples were metallographically prepared with 240, 320, 400,600 , and 1000 grit sandpapers. Then, specimens were polished with $0.3 \mu \mathrm{m}$ alumina and etched with $2 \mathrm{~g}$ of $\mathrm{K}_{2} \mathrm{Cr}_{2} \mathrm{O}_{7}, 8 \mathrm{ml}$ of $\mathrm{H}_{2} \mathrm{SO}_{4}, 4 \mathrm{ml}$ of $\mathrm{NaCl}$ saturated solution, and $100 \mathrm{ml}$ of distilled $\mathrm{H}_{2} \mathrm{O}$.

To measure the hardness of the materials, 16 indentations were practiced on each polished brass sample, according to ASTM E92. One Leco Model LM300AT Vickers microhardness tester at $100 \mathrm{~g}$ load, according to ASTM-E70, was used [17]. The reported Vickers microhardness is shown in Table 1. Thermal diffusivity, conductivity, and heat capacity results of Kunial brass are shown in Table 1.

\subsection{Thermal diffusivity}

An open photoacoustic cell (OPC) was used to analyze the thermal diffusivity of samples [5]. Thicknesses and characteristics of samples are described in Table 1. In experimental setup the samples were placed on top of electret microphone, where a laser (450 nm wavelength) modulated was focused onto the sample surface. Frequency modulation range from 15 to $400 \mathrm{~Hz}$ was used. Photoacoustic technique was used to observe amplitude and phase signal behaviors in the sample [18, 19]. A thermal diffusion model was used to calculate the pressure at the photoacoustic gas

\begin{tabular}{lccccccc}
\hline Sample & $\begin{array}{c}\text { PHT } \\
\text { temperature } \\
{ }^{\circ} \mathrm{C}\end{array}$ & $\begin{array}{c}\text { Thickness } \\
(\boldsymbol{\mu \mathrm { m }})\end{array}$ & $\begin{array}{c}\text { Vickers } \\
\text { microhardness } \\
{[8]}\end{array}$ & $\begin{array}{c}\alpha \\
\left(\mathbf{c m}^{2} / \mathbf{s}\right)\end{array}$ & $\begin{array}{c}\rho \mathrm{C} \\
\left(\mathrm{J} / \mathbf{m}^{3}-\mathrm{K}\right)\end{array}$ & $\begin{array}{c}\boldsymbol{k} \\
(\mathrm{W} / \mathbf{m}-\mathrm{K})\end{array}$ & $\begin{array}{c}\text { Vickers } \\
\text { micro- } \\
\text { hardness } \\
\text { (PTR } \\
\text { technique) }\end{array}$ \\
\hline SHT & NA & 387 & 72 & 0.242 & $3.49 \times 10^{6}$ & 84.46 & 83.7 \\
\hline P300 & 300 & 392 & 82 & 0.463 & $2.60 \times 10^{6}$ & 120.46 & 92.1 \\
\hline P400 & 400 & 375 & 140 & 0.415 & $2.55 \times 10^{6}$ & 105.44 & 121.2 \\
\hline P500 & 500 & 392 & 201 & 0.339 & $2.77 \times 10^{6}$ & 93.32 & 200.6 \\
\hline P600 & 600 & 398 & 162 & 0.508 & $2.42 \times 10^{6}$ & 122.42 & 154.2 \\
\hline P700 & 700 & 401 & 135 & 0.587 & $2.70 \times 10^{6}$ & 158.63 & 133.5 \\
\hline
\end{tabular}

Table 1.

Thermal properties, Vickers microhardness, and thermal diffusivity of Kunial brass samples. 
chamber [20], with the simplification for the thermally thick and optically opaque regime [21] according to the following equation:

$$
V_{O P C}=\frac{A}{f} e^{-\sqrt{\frac{f}{f_{c}}}}
$$

where $\mathrm{A}$ is a constant that contains geometric parameters including factors as gas thermal properties, light beam intensity, and room temperature, $f$ is the frequency scan, and $f_{c}$ is the cutoff which separates thick and thin regimens. The cutoff is related to thermal diffusivity $\alpha$ and the sample thickness $l$ by the following equation [21]:

$$
\alpha=\pi l^{2} f_{c}
$$

\subsection{Heat capacity}

The thermal relaxation method was used to determine the heat capacity [8]. In the experimental setup, a thermocouple was connected to the back side of the sample. A laser light source of $450 \mathrm{~nm}$ wavelength was focused onto the sample surface with a continuous incidence and uniformly. Conduction and convection energy losses were reduced by a vacuum disposal, thereby mainly ensuring a radiation heat transfer [22]. The temperature variation was determined by

$$
\Delta T(t)=\frac{P_{0}}{\eta}\left(1-e^{-\tau / T}\right)
$$

where $\tau$ value is related to the heat capacity by

$$
\rho C_{p}=\frac{8 \varepsilon \sigma T_{0}^{3}}{l_{m}}
$$

and $\varepsilon$ is the thermal emissivity, considered 1 in this case, and $\sigma$ is the Stefan Boltzmann constant. $\mathrm{T}_{0}$ is the temperature, and $\mathrm{l}_{\mathrm{m}}$ is the thickness. The thermal conductivity is related to the heat capacity and thermal diffusivity by

$$
k=\alpha \rho C_{p}
$$

\subsection{Photothermal radiometry (PTR)}

Figure 5 shows the principle of PTR, where a beam of laser light is focused on a material. The radiation absorbed by the material penetrates a certain depth and becomes a wave of heat which propagates through the material. Using an infrared detector, it is possible to detect the signals coming from the thermal wave translated in amplitude and phase. In Figure 6 the experimental setup of photothermal radiometry technique is shown. PTR technique was used to obtain thermal images of alpha brass samples. A high-power semiconductor laser (450 nm wavelength, $300 \mathrm{~mW}$ ) was used. The laser beam was collimated, and then it was focused onto the surface of the sample with a $40 \mu \mathrm{m}$ spot size using a GRADIUM lens. The modulated infrared radiation from the excited surface was collected and collimated by two off-axis paraboloid mirrors, and then, it was focused onto a Judson Model J15D12-M204 HgCdTe detector, which was cooled by liquid nitrogen. The detector signal was amplified by a low-noise preamplifier, and then, it was sent to a lock-in amplifier SRS-850 which was interfaced 
with a PC. XYZ microstages were used to obtain PTR amplitude and phase images $[23,24]$.

An area of $2 \times 2 \mathrm{~mm}$ in each sample was scanned to obtain the thermal images. The PTR technique comprises the optical excitation of the sample with a modulated laser light source and the detection of the recombination-induced infrared emission. PTR technique covers the thermal infrared emission band from 2 to $12 \mu \mathrm{m}$. In PTR the amplitude and phase signals parameters were obtained from a highly focused laser beam, with a waist of $40 \mu \mathrm{m}$.

The thermal wave generated in the sample becomes attenuated at a distance $\mu$, thermal length. Only information due to changes in the thermal properties of the surface of the sample is obtained. The thermal length is defined by

$$
\mu=\sqrt{\frac{\alpha}{\pi f}}
$$

where $\alpha$ is the thermal diffusivity of the sample and $f=\omega / 2 \pi$.

The PTR amplitude signal generated in the sample due to the absorption of modulated laser can be described by the following equation (8):

$$
T(x, t)=\frac{I_{0}}{2 \varepsilon \sqrt{\omega}} \exp \left(-\frac{x}{\mu}\right) \cos \left(\omega t-\frac{x}{\mu}+\frac{\pi}{4}\right)
$$

where $\omega$ is the angular frequency, $\mathrm{I}_{0}$ is the laser intensity, $x$ is the sample thickness, and $\varepsilon$ is the thermal effusivity. The pre-factor in Eq. (10) is constant for a fixed modulation frequency $f=\omega / 2 \pi$.

The PTR amplitude signal is proportional to the reciprocal of the thermal effusivity, while the PTR phase lag is proportional to the $x / \mu$ term. The thermal effusivity and the thermal diffusivity are dependent parameters from the thermal wave propagation which determines the material inertia. The thermal effusivity is a significant heating periodic surface and a heat transport parameter. It is representing the dissipated heat energy in the solid material depending on the temperature change at the beginning of the periodic warming process. The thermal effusivity is related to Eq. (9) by the diffusivity coefficient ( $\alpha$ ), as shown in the following equation:

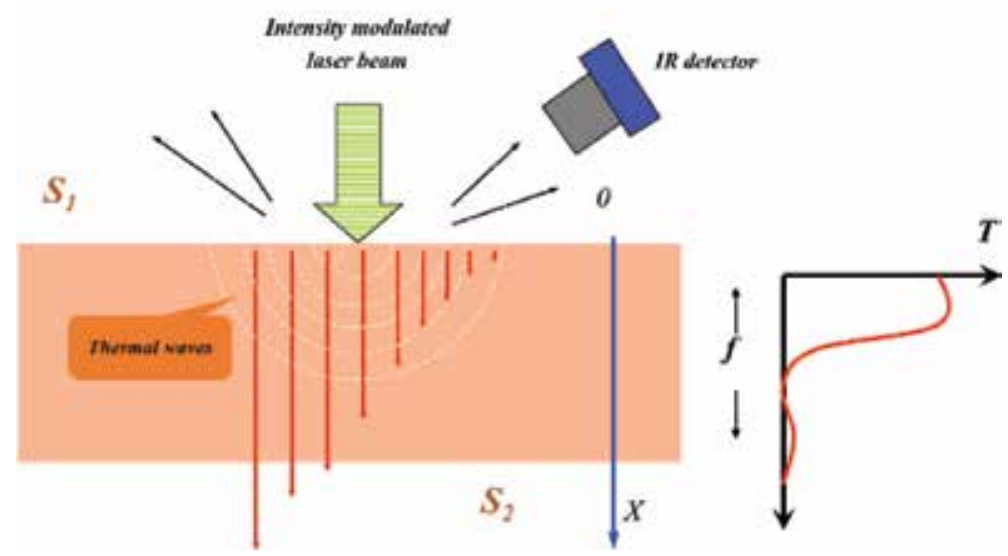

Figure 5.

Principle of PTR technique for characterization of a material. 


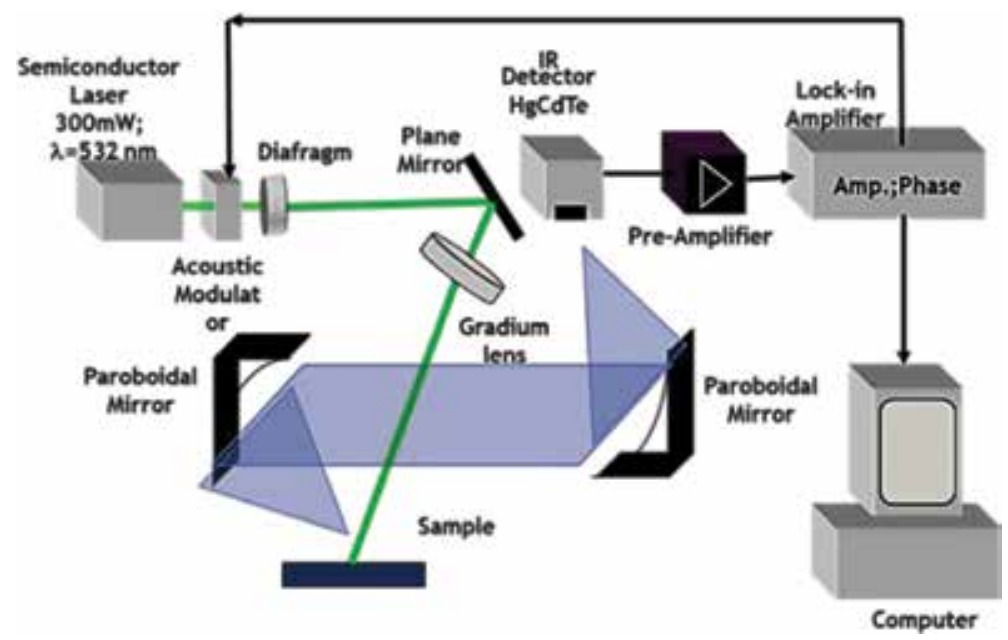

Figure 6.

Experimental setup of PTR technique.

$$
\varepsilon=\frac{k}{\sqrt{\alpha}}=\sqrt{\mathrm{k} \rho c}
$$

where $\mathrm{k}$ is the thermal conductivity, $\rho$ is the material density, and $\mathrm{c}$ is the specific heat at a constant volume.

\subsection{Thermal properties}

Lowest diffusivity and conductivity values that corresponded to the SHT sample are shown in Figures $7 \mathbf{a}$ and $\mathbf{b}$. In Figure $7 \mathbf{c}$ the highest heat capacity value was for the SHT sample. The noticed behavior was that as diffusivity and conductivity increased, the heat capacity trended to decrease. This can be explained by the thermal treatment; due to the temperature increased, the grain size increased, and this had an effect on diffusivity and conductivity. The heat capacity decreased due to the PHT effect because intermetallic precipitates migrated to grain boundaries affecting the heat capacity.

\subsection{Vickers microhardness and FWHM ${ }^{-1}$}

Vickers microhardness results are shown in Figure 8a. The lowest value of Vickers microhardness corresponds to the SHT sample and the highest value to the P500 sample. Due to recrystallization and precipitation phenomena, as the PHT temperature increased, the Vickers microhardness decreased. The precipitation had a little effect from 300 to $400^{\circ} \mathrm{C}$. However, the P500 sample reached the highest Vickers microhardness value at $500^{\circ} \mathrm{C}$. In P600 y P700 samples, the PHT temperature increased, and the microhardness decreased due to over aging.

In Figure $\mathbf{8 b}$ is shown that the $\mathrm{FWHM}^{-1}$ increased as the PHT temperature increased. As the Vickers microhardness decreased, the crystallinity quality increased. The lowest $\mathrm{FWHM}^{-1}$ value was at $400^{\circ} \mathrm{C}$, and then, it trended to increase as the PHT temperature increased. The crystalline quality improved as the PHT temperature increased. In spite of the highest Vickers microhardness value being reached at $500^{\circ} \mathrm{C}(\mathrm{P} 500)$, higher temperatures caused that the Vickers 

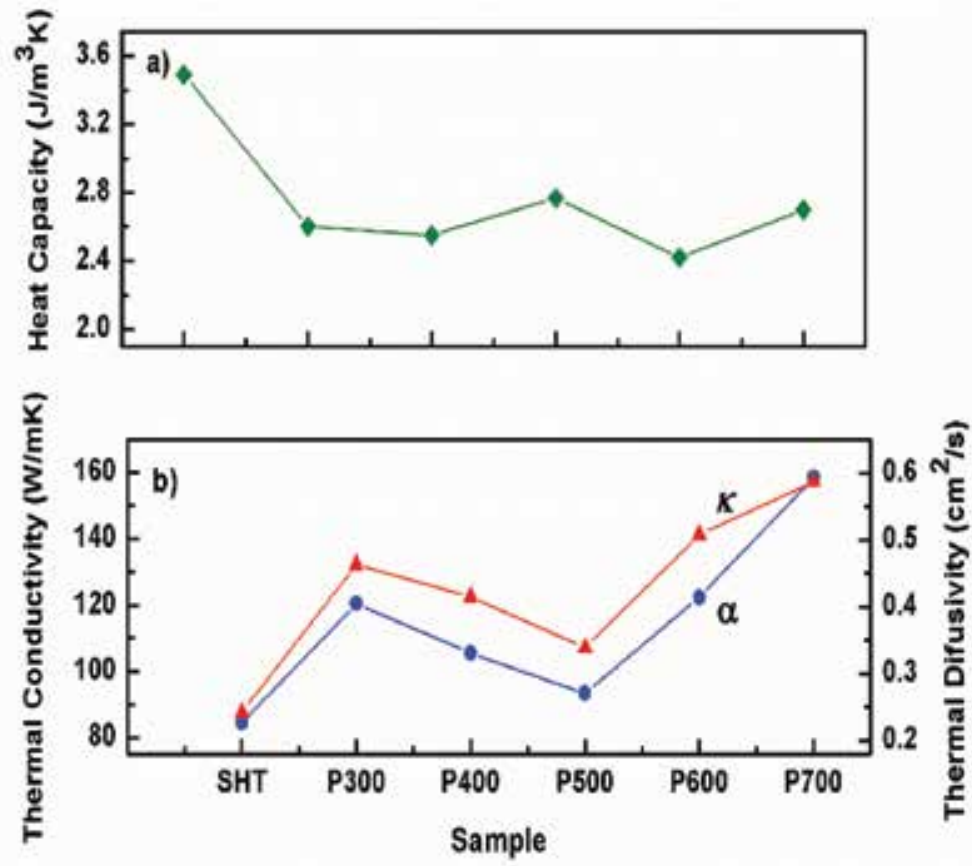

Figure 7.

Thermal properties of Kunial brass samples studied, (a) heat capacity and (b) diffusivity $(\kappa)$ and conductivity $(\alpha)$.

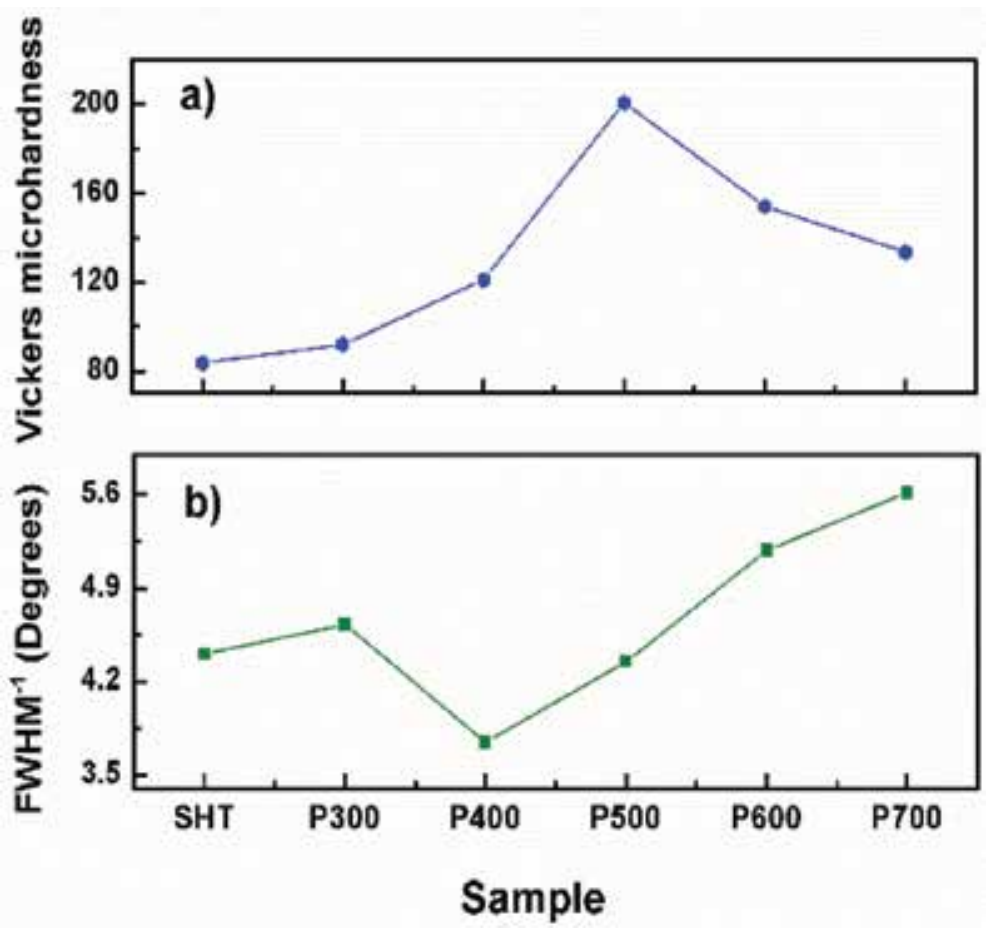

Figure 8.

(a) Vickers microhardness and (b) FWHM ${ }^{-1}$ of brass samples with different thermal properties.

microhardness trended to decrease. A result of a diffusive process at $600^{\circ} \mathrm{C}$ was observed due to the slow cooling temperature. These diffusive processes produced a recrystallization, improving the crystalline quality. 


\subsection{PTR images}

In Figure 9 PTR images are shown. In Figure 9a, it is shown that the amplitude increased as the PHT temperature increased until the highest signal of $500^{\circ} \mathrm{C}$. Subsequently, amplitude signals decreased as the temperature increased until the lowest value of $700^{\circ} \mathrm{C}$. However, no large phase signal changes were observed in Figure 9b.

The highest Vickers microhardness and the optimum precipitation were presented in the P500 sample. The lowest Vickers microhardness was obtained in the P700 sample. The comparison among the Vickers microhardness and PTR amplitude and phase signals is shown in Figure 10. Vickers microhardness trend is shown in Figure 10a. Figure 10b illustrates the amplitude behavior was similar to the hardness pattern, because the PTR signal was related to brass structural changes. The maximum PTR amplitude signal was reached at $500^{\circ} \mathrm{C}$ that is the optimum PHT temperature which corresponded to the maximum Vickers microhardness of the studied brass [25]. It is possible to observe in Figure 10c, that the phase signal
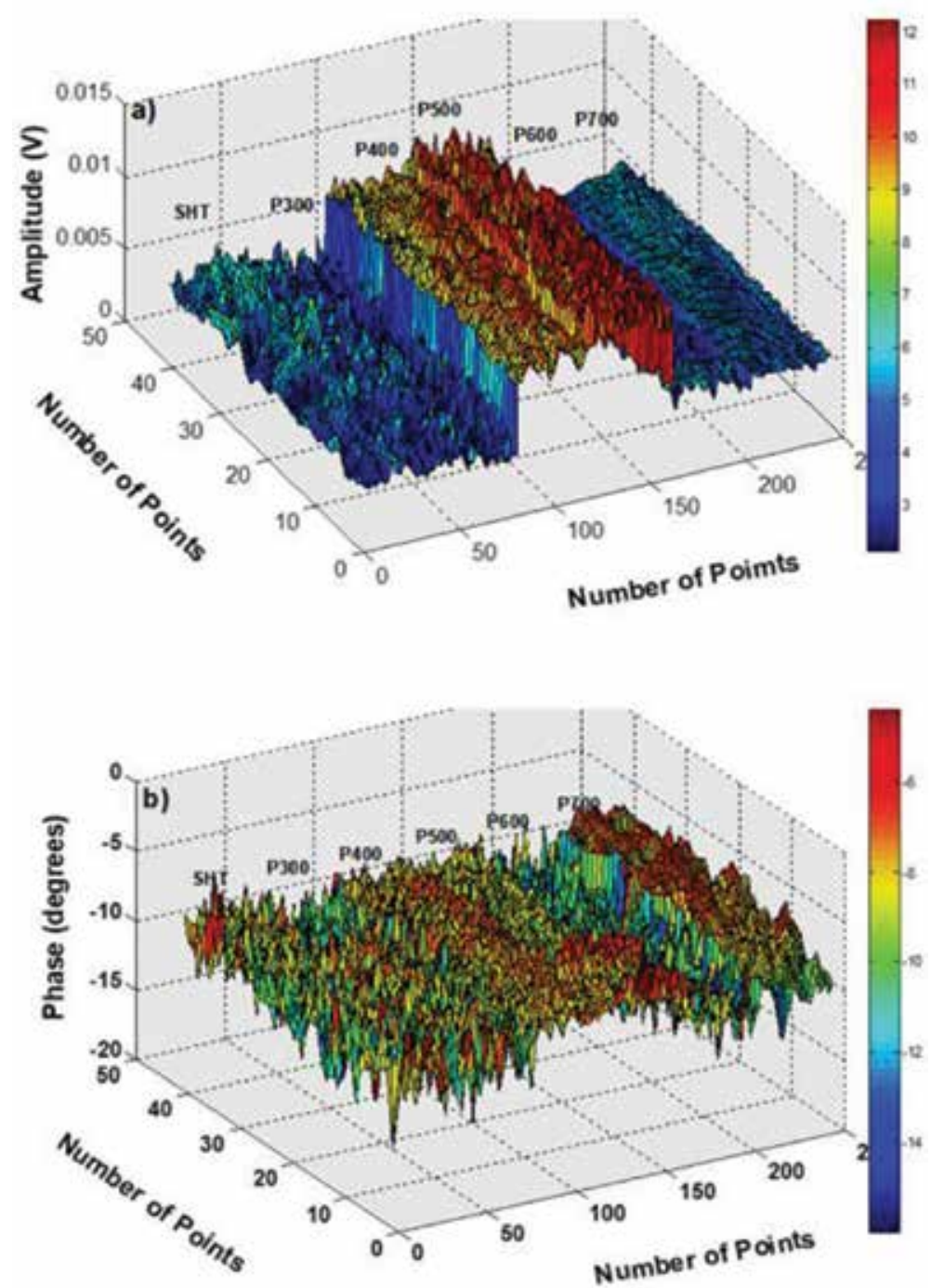

Figure 9.

Photothermal images experimental of brass samples, (a) amplitude and (b) phase. 
behavior did not show large changes as in the amplitude signal. The highest PTR phase signal corresponded to the SHT sample, while the lowest PTR phase signal corresponded at $700^{\circ} \mathrm{C}$. This means that the PTR amplitude signal was more sensitive than the PTR phase signal, to observe the effect of the PHT temperature on $\alpha$ brass thermal, structural, and Vickers microhardness properties.

\section{Conclusions}

The finite element method (FEM) offers an option for the solution of complex problems in engineering with a multiphysical profile and equations difficult to solve analytically. The model presented in this chapter, and developed on the COMSOL
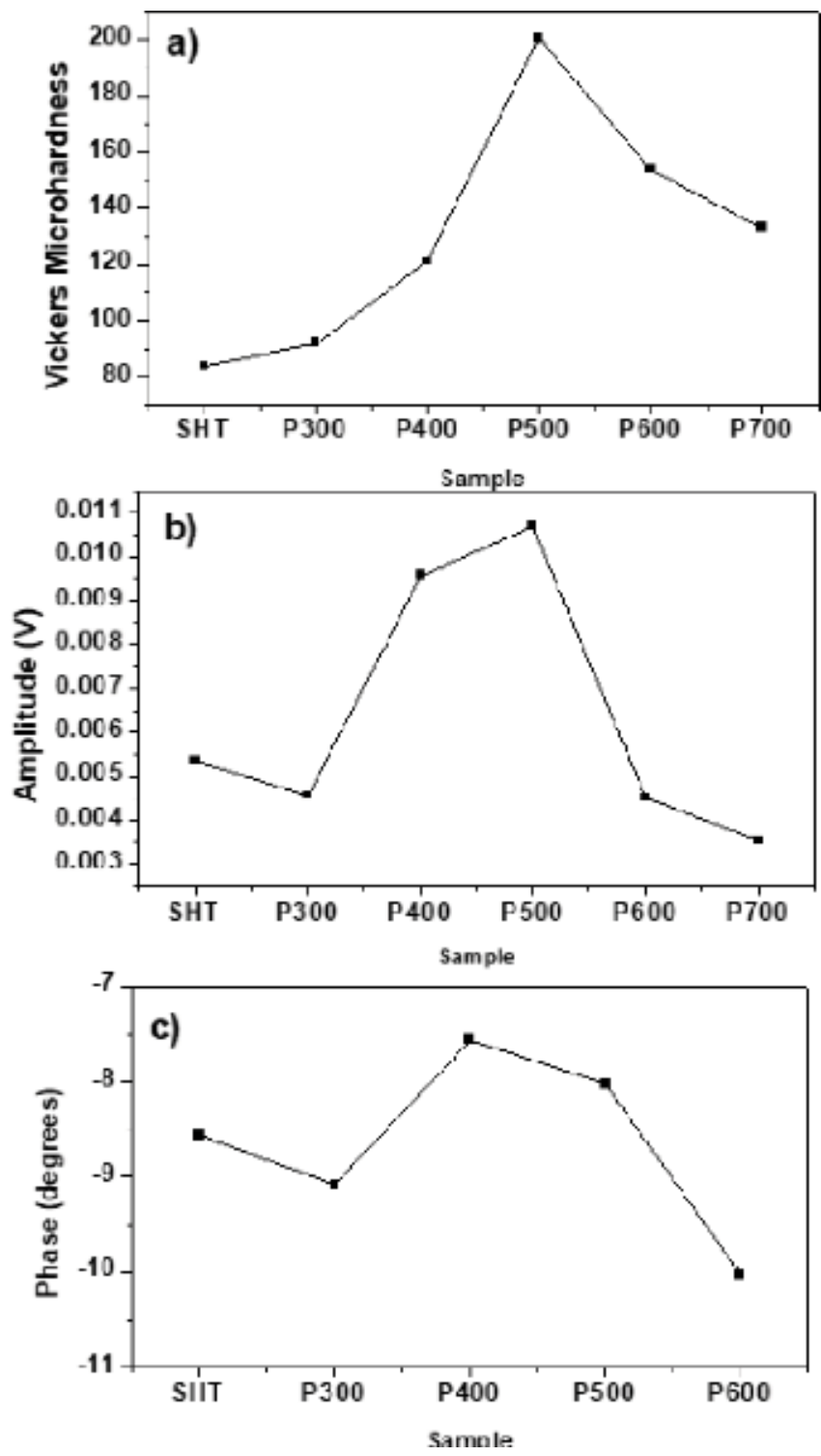

Figure 10.

Comparison among (a) Vickers microhardness and (b) amplitude and (c) phase signals of PTR applied to brass samples. 
Multiphysics platform, is a basic approach to the application of the law of LambertBeer applied to the phenomenon of the absorption of laser light in a material. Due to the multiphysics profile of the program, it is possible to complement the model with other modules of the platform as geometrical optical to modified characteristics of laser beam incident in the material.

In the program it is considered that the definition of the mesh is one of the key points to obtain a good simulation that its erroneous choice the design of the same can cost the execution time of the program, or in the worst of the cases a bad convergence, translated into erroneous results.

The phenomenon of light absorption influences materials differently, depending on their physical-chemical properties such as thermal diffusivity, density, conductivity, and heat capacity. In the experimental results shown, it can be seen that the thermal treatments that involve the absorption phenomenon induce changes in the mechanical properties of the materials, such as microhardness. As reported in the Kunial brass samples analyzed, the PHT affected its metallurgical, thermal, and mechanical properties.

A marked correlation was found between the FWHM and the diffusivity. The diffusivity increased as the FWHM-1 increased. The highest diffusivity corresponds to $700^{\circ} \mathrm{C}$ while the lowest to the SHT sample.

The PTR amplitude signal was more sensitive than the PTR phase signal to observe the effect of the PHT temperature on alpha brass thermal, structural, and Vickers microhardness properties.

It is clear that the theoretical results obtained in COMSOL describe temperature variations as a function of the penetration depth of the incident radiation in the material, which in turn depends on factors such as the incident power. Because in the photothermal radiometry technique the thermal wave captured by the detector is translated as amplitude and phase signals, it is not possible to correlate both results. However, it is clear that a finite element analysis such as that offered by COMSOL offers the advantage of inferring the behavior of the variables involved in the process.

Among the possible improvements to the program are the inclusions of the modulation of the light source, which is a very important factor in the photothermal radiometry technique (PTR). The thermal wave generated in the material is very weak and necessarily requires an amplification. Without the modulation of the light source, the amplitude and phase parameters describing the thermal wave would not be possible to capture due to the amount of interference present in the medium. It is important to mention that the modulation frequency of the incident beam directly influences the penetration length in the material and consequently the propagation of the thermal wave.

With a more detailed theoretical-experimental analysis, it is possible to arrive at a comparable result between both parties, either by making measurements directly from temperature variations in the samples analyzed or by defining the variables and conditions in the program that allow obtaining the parameters of amplitude and phase of the generated thermal wave.

\section{Acknowledgements}

The authors thank Mario Enrique Rodríguez-Garcia of the Center of Applied Physics and Advanced Technology (CFATA, UNAM) for the support received for carrying out the experiments, the program Cátedras-CONACYT, and the National Council for Science and Technology (CONACyT) for funding through project 256923. 


\section{Author details}

Minerva Robles-Agudo*, Martha Patricia Gonzalez-Tejeda, Porfirio Esau Martinez-Muñoz and Ignacio Rojas-Rodríguez CONACYT-Technological University of Querétaro (UTEQ), Querétaro, México

*Address all correspondence to: minerva.robles@uteq.edu.mx

\section{IntechOpen}

(C) 2019 The Author(s). Licensee IntechOpen. This chapter is distributed under the terms of the Creative Commons Attribution License (http://creativecommons.org/licenses/ by/3.0), which permits unrestricted use, distribution, and reproduction in any medium, provided the original work is properly cited. (cc) BY 


\section{References}

[1] Copper Development Association. Brass in Focus 2004;177:1-4. Available from: http://copperalliance.org.uk/ docs/librariesprovider5/resources/ pub-177-brass-in-focus-pdf.pdf?Status $=$ Master\&sfvrsn $=0$

[2] Khurmi RS, Gupta JK. A Textbook of Machine Design. 2004;30:6. Available from: https://es.slideshare. net/alianzam900/a-textbook-ofmachine-design-by-rskhurmi-an-djkgupta-44120476

[3] Waheed A, Ridley N. Microstructure and wear of some high-tensile brasses. Journal of Materials Science. 1994;29:1692-1699. DOI: 10.1007/ BF00368948

[4] Sadykov FA, Barykin NP, Aslanyan IR. Wear of copper and its alloys with submicrocrystalline structure. Wear Volumes. 1999;225-229:649-655. DOI: 10.1016/S0043-1648(98)00374-3

[5] Panagopoulos CN, Georgiou EP, Simeonidis K. Lubricated wear behavior of leaded $\alpha+\beta$ brass. Tribology International. 2012;50:1-5. DOI: 10.1016/j.triboint.2011.12.016

[6] Ozgowicz W, Kalinowska EO, Grzegorczyk B. The microstructure and mechanical properties of the alloy $\mathrm{Cu}-30 \% \mathrm{Zn}$ after recrystallization annealing. Journal of Achievements in Materials and Manufacturing Engineering. 2010;40:15-25. Available from: http://jamme.acmsse.h2.pl/ papers_vol40_1/4012.pdf

[7] Igelegbai EE, Alo OA, Adeodu AO, Daniyan IA. Evaluation of mechanical and microstructural properties of a $\alpha$-brass alloy produced from scrap copper and zinc metal through sand casting process. Journal of Minerals and Materials Characterization and Engineering. 2017;5:18-30. Available from: https://file.scirp.org/pdfJMMCE_2016122014370253.pdf. DOI: 10.4236/jmmce.2017.51002

[8] Bailey AR. The Structure and Strength of Metals, Annotated Metallographic Specimens. Betchworth: Gatwick Press Ltd.; 1967. pp. 75-88. Available from: http://www.worldcat. org/title/structure-and-strength-ofmetals-an-elementary-practic-alcourse-involving-th-correlation-ofmicrostructure-an-properties-in32-specially-prepared-specimens/ oclc/299573857

[9] Allmen MV, Blatter A. LaserBeam Interactions with Materials, Physical Principles and Applications. 2nd ed. Springer; 1998. Available from: https://www.springer.com/gp/ book/9783642970078

[10] COMSOL Multiphysics. Blogs. 2015. Available from: https://www.comsol. com/blogs/modeling-laser-materialinteractions-with-the-beer-lambert-law/

[11] Buijs K, Maurice MJ. Some considerations on apparent deviations from Lambert-Beer's law. Analytica Chimica Acta. 1969;47(3):469-474. DOI: 10.1016/S0003-2670(01)95647-8

[12] Tolbin AY, Pushkarev VE, Tomilova LG. A mathematical analysis of deviations from linearity of Beer's law. Chemical Physics Letters. 2018;706(16):520-524. DOI: 1016/j. cplett.2018.06.056

[13] Casasanta G, Garra R. Towards a generalized Beer-Lambert law. Fractal and Fractional. 2018;2:8. DOI: 103390/ FRACTALFRACT2010008

[14] Moosmüller H, Arnott WP. Particle optics in the Rayleigh regime. Journal of the Air and Waste Management Association. 2009;59:1028-1031. DOI: 10.3155/1047-3289.59.9.1028 
[15] Uriarte LM, Bonales LJ, Dubessy J, Lobato A, Baonza VG, Cáceres M. The self-absorption phenomenon in quantitative Raman spectroscopy and how to correct its effects. Microchemical Journal. 2018;139:134-138. DOI: 10.1016/J.MICROC.201802.013

[16] COMSOL Multiphysics. Available from: https://www.comsol.com

[17] ASTM E92-82(2003) e2. Standard Test Method for Vickers Hardness of Metallic Materials, Subcommittee: E28.06, ASTM International; 2003. pp. 1-9

[18] Dias DT, Bedeschi VC, Ferreira da Silva A, Nakamura O, Castro-Meira MV, Trava-Airoldi VJ. Photoacoustic spectroscopy and thermal diffusivity measurement on hydrogenated amorphous carbon thin films deposited by plasma-enhanced chemical vapor deposition. Diamond and Related Materials. 2014;48:1-5. DOI: 10.1016/J. diamond.2014.06.003

[19] Rojas-Rodríguez I, Lara-Guevara A, Salazar-Sicacha M, Mosquera-Mosquera JC, Robles-Agudo M, RamírezGutierrez C, et al. The influence of the precipitation heat treatment temperature on the metallurgical, microstructure, thermal properties and microhardness of an alpha brass. Materials Sciences and Applications. 2018;9:440-454. DOI: 10.4236/ msa.2018.94030

[20] Lara-Guevara A, Rojas-Rodríguez I, Ortiz-Echeverri CJ, Robles-Agudo JM, Rodríguez-García ME. Thermal, structural, and microstructural characterization of eutectoid steel at different heat treatments. Journal of Materials Research. 2017;32:2202-2209. DOI: 10.1557/jmr.2017.47

[21] Lara-Guevara A, Ortiz-Echeverri CJ, Rojas-Rodriguez I, Mosquera-Mosquera JC, Ariza-Calderón H, Ayala-García I, et al. Microstructural, structural, and thermal characterization of annealed carbon. International Journal of Thermophysics. 2016;37(10):99. DOI: 10.1007/s10765-016-2105-6

[22] Demkó L, Kézsmárki I, Csontos M, Bordács S, Mihály G. Improved thermal relaxation method for the simultaneous measurement of the specific heat and thermal conductivity. European Physics Journal B. 2010;74:27-33. DOI: 10.1140/ epjb/e2010-00055-0

[23] Rojas-Rodríguez I, JaramilloVigueras D, Velázquez-Hernández R, Del Real A, Serrounkh I, Baños $\mathrm{L}$, et al. Thermal and structural characterization of cooper-steel bonding interfaces produced by impact welding. Materials and Manufacturing Processes. 2008;23:823-828. DOI: 10.1080/10426910802384672

[24] Rosencwaig A, Gersho A. Theory of the photoacoustic effect with solids. Journal of Applied Physics. 1976;47: 64-69. DOI: $10.1063 / 1.322296$

[25] Guo X, Sivagurunathan K, Garcia JA, Mandelis A, Giunta A, Milletari $S$. Laser photothermal radiometric instrumentation for fast in line industrial steel hardness inspection and case depth measurements. Applied Optics. 2009;48:11-22. DOI: 10.1364/ AO.48.000C11 


\title{
Discrimination by Infrared Spectroscopy: Application to Micronized Locust Bean and Guar Gums
}

\author{
Catherine Rébufa, Fabien Girard, Jacques Artaud \\ and Nathalie Dupuy
}

\begin{abstract}
The authentication of locust bean and guar powder gums requires usually the use of sophisticated and time-consuming analytical techniques. There is a need for fast and simple analytical techniques for the objective of a quality control methodology. Commercial locust bean and guar micronized powder gums present characteristic MIR spectra. Principal component analysis of the infrared spectra of these micronized powder gums allowed to distinguish locust bean from guar samples and to perform good classification results. The prediction of the two varieties was done without any ambiguity with a partial least square regression-discriminant analysis (PLS-DA). A simplex approach was used to generate binary blends mathematically taking into account the intrinsic variability of chemical composition of commercial products. The simulated spectral profiles allowed to develop predictive model of the percentage of gums in blends.
\end{abstract}

Keywords: locust bean, carob, guar, infrared, chemometrics, classification

\section{Introduction}

In food industry, it is common to add ingredients that have in aqueous solution thickening, stabilizing, or gelling properties to the formulation of food products. These ingredients are interesting for the appearance and organoleptic characteristics but can also become innovative ingredients in the search for original textures or to support the use of alternative chemical additives. In bioprocessing, the field of ingredients or additives authorized in this framework is more limited than in conventional processing. However, a number of gums, starches, fibers, or flours can be used. Among them, two vegetable gums, extracted from the endosperm of legume seeds, were commonly used with similar properties: guar gum and locust bean gum. These natural polysaccharides are extensively used in a wide range of applications in food, medical, pharmaceutical, textile, paper, hydraulic fracturing, explosives, agriculture, cosmetic, bioremediation, and petroleum industries because of their ability to modify the rheological properties and in the aim of green chemistry approach [1-5]. Guar gum (GG), named E412 in European 
additive list, is a polysaccharide of natural origin, extracted from the seed of Cyamopsis tetragonoloba L. (Fabaceae), a plant also called guar or guar bean and native. The world's production of guar is concentrated in India, Pakistan, and the United States with limited amounts grown in South Africa and Brazil. The annual guar plant grows to about $0.6-1 \mathrm{~m}$ in height and produces seed pods growing in clusters giving guar pods the common name cluster bean (6-9 guar beans per pod). Locust bean gum (LBG) or E410 is the crushed endosperm of locust bean tree seeds, a dioecious evergreen tree grown in a Mediterranean climate, botanically known as Ceratonia siliqua L., belonging to the subfamily Caesalpinioideae of the Fabaceae family. It is abundant in Spain, Italy, and Cyprus and is also found in other Mediterranean countries, in various regions of North Africa, South America, and Asia. It can reach $8-15 \mathrm{~m}$ in height and live up to 500 years. Its fruits are long thick and tough pods containing 10-15 oval-shaped locust bean seeds or kernels. The locust tree can produce annually $300-800 \mathrm{~kg}$ of locust bean seeds from which LBG will be produced, also referred as locust bean seed gum, locust bean flour, or ceratonia. The guar seed is smaller than the locust bean seed but they both have the same structure. They consist of four elements (Figure 1): the tegument (outer husk or seed coat), a translucent endosperm representing $40-50 \%$ of the weight of the locust bean seed and 35-45\% of the weight of the guar seed, two cotyledons, and an embryo (or germ) [6].

The number of tissues within the locust bean would even be 10 according to microscopic studies [7]. These seeds are a basic material for the manufacture of gum and contain hydrocolloids (polysaccharides), called galactomannans, which serve as a reserve for the embryo during germination. With the help of various thermal, mechanical, or chemical processes $[2,5]$, the seeds are dehusked without damaging the endosperm and the embryo (germ). After this peeling process, the endosperm is split from the cotyledons and then it is ground to produce gum. To eliminate protein content and impurities for certain industrial applications, the gum is purified by washing with solvent or dispersing in boiling water, followed by filtering, evaporation, and drying $[2,5,6,8]$. Galactomannans are heterogeneous polysaccharides with a high molecular weight, composed by linear chain of $\beta$-(1-4)-D-mannopyranosyl units with a single $\alpha$-D-galactopyranosyl (1-6) linked residue, a conformation similar to that of cellulose. The structure of these gums differs according to the distribution and the number of galactose residue along the mannose chain, randomly arranged in pairs and triplets in the case of the GG [5] leading to regions of low or high substitution, or in blockwise while LBG presents a random, blockwise, and ordered distribution of $\alpha$-D-galactopyranosyl residues along the $\beta$-D-mannose backbone [9]. The degree of galactosyl substitution is responsible for water solubility differences of galactomannans; an increase in the substitution leads to higher solubility through steric effects, whereas galactose-poor regions are less soluble and can involve both inter- and intramolecular associations. Then, GG is dissolved in cold water, while heating is needed to solubilize LBG. The

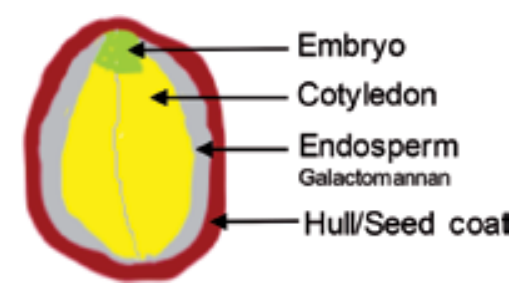

Figure 1.

Scheme of gum seed cross-section. 
ratio of mannose to galactose can vary because of the extraction process (particularly purification conditions depending of the end of the desired product) and plant source (geographic location with various climate). The average ratio of galactose to mannose has been estimated to be $1 / 2$ for GG (typically in the range $1 / 1.4-1.8$ ) $[2,10,11]$ and $1 / 4$ (found in the range $1 / 2.3-6.0$ ) $[2,11,12]$ for $L B G$ according to the different chemical techniques (high-performance liquid chromatography, gas chromatography, ${ }^{13} \mathrm{C}$ NMR spectroscopy, or enzymatic method with $\beta$-D-mannase).

Because of its good sensitivity and its simplicity in sample preparation, Fourier Transform InfraRed (FTIR) spectroscopy has been common to differentiate GG and LBG and mixtures $[10,12,13]$, to study galactomannose interaction with solids [14], to control the gum quality after chemical treatments modifying their properties $[15,16]$, and to predict the origin [17] with a partial least squares regression-discriminant analysis (PLS-DA).

The aim of this work is to confirm the potential of FTIR technique for the discrimination and the classification of the nature of LBG and GG with the help of chemometric treatments such as principal component analysis (PCA) and partial least squares regression. Moreover, linear-discriminant analysis (LDA) was used to predict the percentage of adulteration by using Scheffé's simplex network to generate simulated binary blends taking into account the variability of the chemical composition of GG and LBG because of their different geographic origins and manufacturing processes.

\section{Materials and method}

\subsection{Sampling}

Guar $(n=74)$ and locust bean $(n=25)$ commercial gums were obtained from different suppliers without information about their geographic origins, manufacturing processes, mannose/galactose ratios, and the mesh size of particles (Table 1).

These powders were freeze-dried before spectroscopic characterization to eliminate the available water interactions. Mathematical binary blends were also built with simplex approach in different GG percentages (varying between 0 and 100\% in weight) from simplex method to take into account the variability of spectral signature of GG and LBG.

Pure sugars (D-mannose and D-galactose) were purchased from Sigma-Aldrich (99\% of purity) to obtain its FTIR-ATR profile in the same conditions of the gum sample.

\subsection{Attenuated total reflectance (ATR) characterization}

The technique of attenuated total reflectance (ATR) is making easier the solid and liquid analysis by reducing the sample preparation time and increasing spectral

\begin{tabular}{cl}
\hline Gum & Suppliers \\
\hline LBG & $\begin{array}{l}\text { Alliance Gums \& Industries, ARLES, Chemcolloids Ltd, Iranex, Pharmacie des Rosiers, Santeflor, } \\
\text { SEATH International, Sigma Aldrich, Tassy \& Cie, Viscogum FA }\end{array}$ \\
\hline \multirow{2}{*}{ GG } & $\begin{array}{l}\text { Alliance Gums \& Industries, ARLES, Chemcolloids Ltd, Associated Dichem corporation, Iranex, } \\
\text { Laviosa MPC, Nitrochemie, SEATH International, Sigma Aldrich, Santeflor, Starlight, ROTH, } \\
\\
\text { Tassy \& Cie, Viscogum FA }\end{array}$ \\
\hline
\end{tabular}

Table 1.

Gum suppliers. 


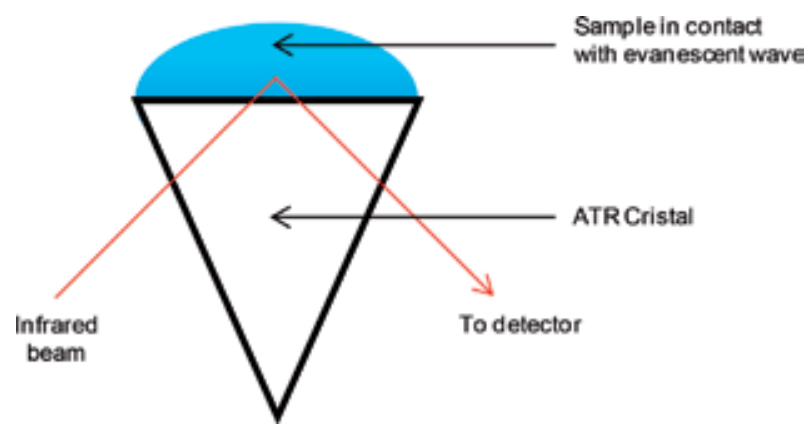

Figure 2.

A simple reflection ATR system.

reproducibility by depositing the sample on the crystal of the attenuated total reflection accessory. By crossing the optical dense crystal (with a high refractive index), the infrared beam will undergo the phenomenon of total internal reflection creating an evanescent wave that extends beyond the surface of the crystal and penetrates a few microns into the sample (the least dense medium). If the sample absorbs light, a part of light energy is retained and the total reflection is attenuated (Figure 2).

The penetration depth $D_{p}$ of the evanescent wave at any specific wavelength is a function of the angle $\phi$ of incidence of the internally reflected beam and of the ratio of the refractive index $n_{1}$ of the crystal to the index $n_{2}$ of the sample:

$$
\mathrm{D}_{\mathrm{p}}=\lambda /\left[2 \pi \mathrm{n}_{1}\left(\sin ^{2} \phi-\left(\mathrm{n}_{2} / \mathrm{n}_{1}\right)^{2}\right)\right]^{1 / 2}
$$

The refractive index of a diamond crystal is $\mathrm{n}_{1}=2.4$ and for the organic compound $n_{2}=1.5$ on the average. For an angle of incidence of $45^{\circ}$, the penetration depth is approximated by $\mathrm{D}_{\mathrm{p}}=0.2 \lambda$ (i.e., between 0.5 and $5 / \mu \mathrm{m}$ approximately for the mid-infrared range). This ATR experiment supposes a very good optical contact between the crystal and the sample. To improve this contact, a press is used.

GG and LBG powders were directly deposited on the attenuated total reflectance (ATR) accessory (Specac's "Golden Gate”) equipped with a diamond crystal prism (brazed in only one tungsten carbide part), four mirrors, and two $\mathrm{ZnSe}$ focusing lenses to reflect the optical path. The sample was pressed on the crystal area with the pressure arm. FTIR-ATR spectra were recorded with a Thermo Nicolet IS10 spectrometer equipped with a Mercury Cadmium Telluride (MCT) detector, an Ever-Glo source, and a $\mathrm{KBr} / \mathrm{Ge}$ beam-splitter, at room temperature. Data acquisition was done in absorbance mode from 4000 to $650 \mathrm{~cm}^{-1}$ with a $4 \mathrm{~cm}^{-1}$ nominal resolution (OMNIC 8.1 software). For each spectrum, 100 scans were co-added. A background scan in air (in the same resolution and scanning conditions used for the samples) was carried out before the acquisition. The ATR crystal was carefully cleaned with ethanol to remove any residual trace of the previous sample. Three spectra were recorded for each sample.

\subsection{Spectral corrections}

The spectral range of the absorption of the carbon dioxide was removed (between 2400 and $1900 \mathrm{~cm}^{-1}$ ) and then a baseline correction was used to adjust the spectral offset. A unit vector normalization was applied to compensate for additive and/or multiplicative effects (Figure 3). 

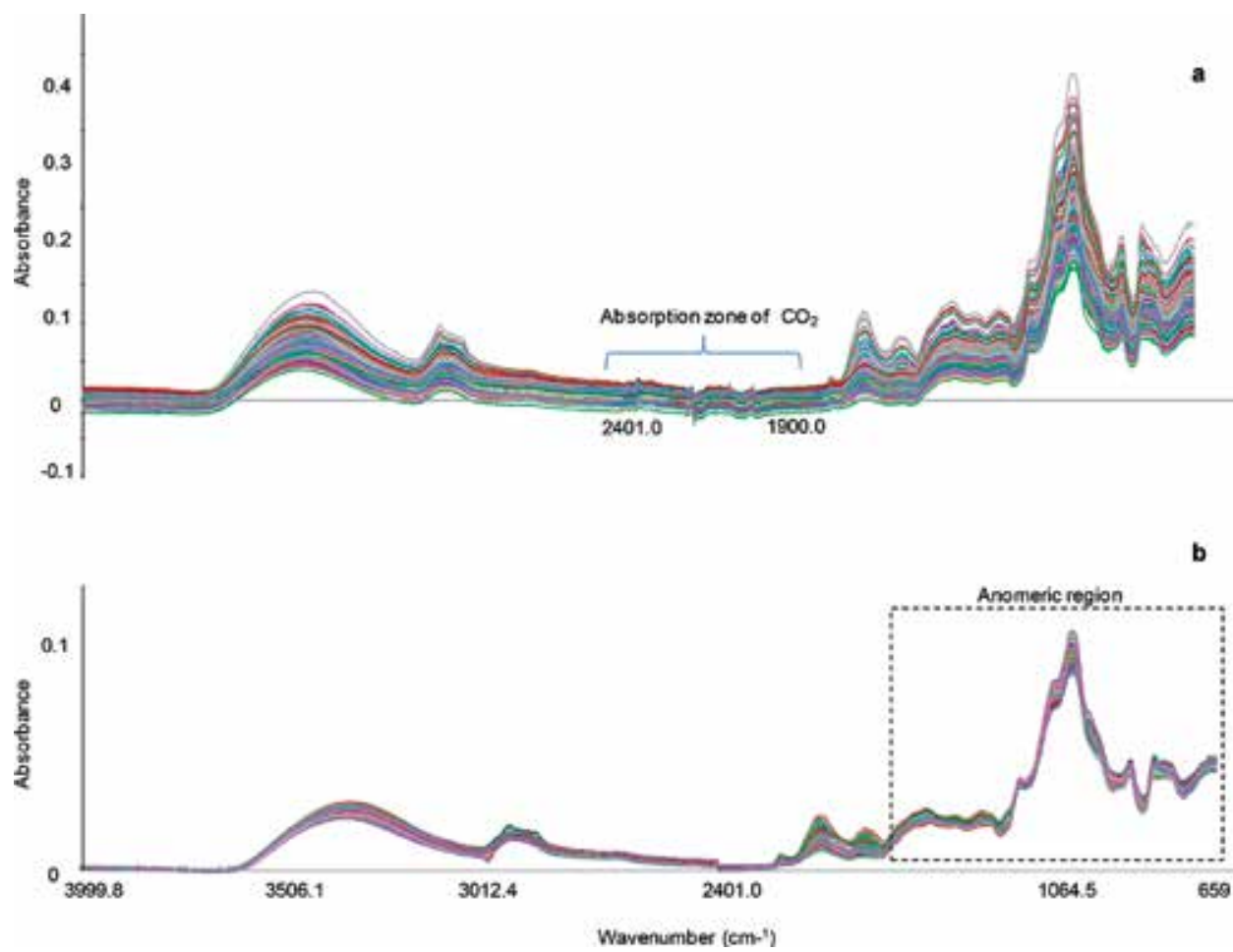

Figure 3.

Guar and locust bean gum FTIR-ATR spectra: original data (a) and corrected data (b).

A selection of variable was used between 1450 and $650 \mathrm{~cm}^{-1}$ for the chemometric treatments to keep only the fingerprint of gums where the anomeric region was located.

\subsection{Chemometrics}

Principal component analysis (PCA) and partial least squares discriminant analysis (PLS-DA) regression are two tools currently used in chemometrics, they are described in a previous study $[18,19]$. The optimal number of principal components and latent variables was determined by a cross validation. A binary codification was used to build PLS-DA models which will predict continuous values (positive or negative) and arbitrary intervals of predicted values were chosen as zones delimited the predicted samples well and no-recognized. Also samples with a reference value of one (expected positive) were considered true positive if their predicted value was between 0.7 and 1.3, false negative if predicted under 0.5 , and uncertain if predicted between 0.5 and 0.7 or over 1.3 . On the contrary, samples with a reference value of zero (expected negative) were considered true negative if predicted between -0.3 and 0.3 , false positive over 0.5 , and uncertain between 0.3 and 0.5 or under -0.3 . The quality of the model was evaluated by the total percentage of correct classification, as well as sensitivity and specificity, calculated according to the following equations:

$$
\begin{gathered}
\text { \%correct classification of variety }=\frac{\text { true positive }+ \text { true negative }}{\text { total number of predicted samples }} \times 100 \\
\text { \%sensitivity }=\frac{\text { true positive }}{\text { expected positive }} \times 100
\end{gathered}
$$




$$
\% \text { specificity }=\frac{\text { true negative }}{\text { expected negative }} \times 100
$$

Linear discriminant analysis (LDA) is the simplest of all possible classification methods that provide a linear transformation of $n$-dimensional samples into an $\mathrm{m}$-dimensional space $(\mathrm{m}<\mathrm{n})$. LDA allowed to develop a model based on predefined classes (GG proportion-LBG proportion) and corresponding FTIR-ATR data of pure gums and gum blends. Here calibration model was built with gum blends generated mathematically from Scheffés simplex approach (described in the following section) in order to obtain a large number of combinations of gum proportions with different increments (between 0 and 100\%) and determine the best fit parameters for classification of gums. These models were then used to classify pure gums or blends not considered in the calibration step. For models, the percentage of recognition (or correct classification) of gum proportions was obtained by calculating first the absolute error on the proportion of each gum as follows:

$$
\text { Absolute error }=\mid \text { proportion }^{\text {predicted }}-\text { proportion }\left.^{\text {reference }}\right|_{\text {guar or locust bean gum }}
$$

A correct classification was considered when absolute error on gum proportion was inferior to the increment and a value of one was attributed to the sample. Otherwise, a zero value was affected to samples with badly predicted gum proportion. Then a percentage of correct classification was calculated.

\subsection{Simplex mixture system}

The dataset of binary mixtures was built with a simplex approach [20] where the studied factors were the proportions of the components. FTIR-ATR spectral data of GG and LBG powders were combined in several proportions given by Scheffé's mixture design [21, 22] to create artificial blends. Considering q components and a constant constraint on the sum of component proportions, Scheffé used a regular $(q-1)$ dimensional simplex to describe the experimental region of the possible component combinations. In our case, a binary system $(q=2)$, the required simplex is a straight line, where $q$ apexes of simplex space corresponded to pure component (GG or LBG). On this linear space, each of the $N$ calculated mixtures was characterized by a weight $\left(\mathrm{w}_{\mathrm{j}}\right)$ satisfying the following equations:

$$
\begin{gathered}
\sum_{j=1}^{\mathrm{q}=2} \mathrm{w}_{\mathrm{j}}=1 \\
\mathrm{~N}=\frac{(\mathrm{w}+\mathrm{q}-1) !}{(\mathrm{q}-1) ! \mathrm{w} !}
\end{gathered}
$$

This method favored a uniform distribution of each mixture between the two pure components and made sure that all these immediate neighbors were at the same distance from the latter. The constant $\mathrm{w}$ represented here the mesh step.

An approach similar to that developed by Semmar et al. [20] was applied to take into account the variability of the spectral profile of pure components due to different geographic origins or different manufacturing processes. Spectral profiles of pure component were randomly chosen between the dataset of pure GG or LBG samples to obtain an average profile subsequently used to build mixture components. The number of spectral data of pure components was equal to w, defined previously. $k$ iterations (400) of Scheffé's simplex design were carried out to ensure variability in average spectral profiles. A matrix of $(\mathrm{N} \times \mathrm{k})$ mixtures was obtained with variables corresponding to the spectral wavenumbers and the associated values 
of $w_{j}$. These average mixture profiles were used as input data to perform a linear discriminant analysis to build a calibration model to predict further the pair of points $\left(\mathrm{w}_{\mathrm{GG}}, \mathrm{w}_{\mathrm{LBG}}\right)$ representing the proportions of each gum in the blends.

\subsection{Software}

The Unscrambler Version 10.3 from CAMO (Computer Aided Modeling, Trondheim, Norway) was used to perform chemometric analyses (PCA, PLS, and LDA). The Scheffé approach was developed using MATLAB R2014b.

\section{Results and discussion}

\subsection{Spectral signature of GG and LBG}

Galactomannans are polysaccharides formed by a linear (1-4)- $\beta$-D-mannan backbone with a D-galactose side chain (Figure 4). On average, GG has a single $\alpha$-D-galactopyranosyl unit connected by (1-6) linkages to every second main chain unit. In the case of the LBG, unsubstituted or sparingly (1-4) substituted regions of mannopyranose units and regions heavily substituted with $\alpha$-D-galactopyranosyl residues attached by (1-6)-bonds have been observed.

The galactose substitution that influences the intrinsic flexibility of mannan backbone causes solubility differences and controls the rheological properties. The FTIRATR signatures of GG and LBG (Figure 5) show essentially difference in intensity due to the mannose/galactose ratio and the presence of residual chemical compounds from thermo-mechanical and chemical dehusking pretreatments (remaining germ particles, products of thermal degradation of endosperm, etc.). In GG, the endosperm is composed of $75 \%$ of galactomannose and the rest consists of pentosan, protein, pectin, phytin, ash, and dilute acid insoluble residues [5]. High protein content (like albumin,

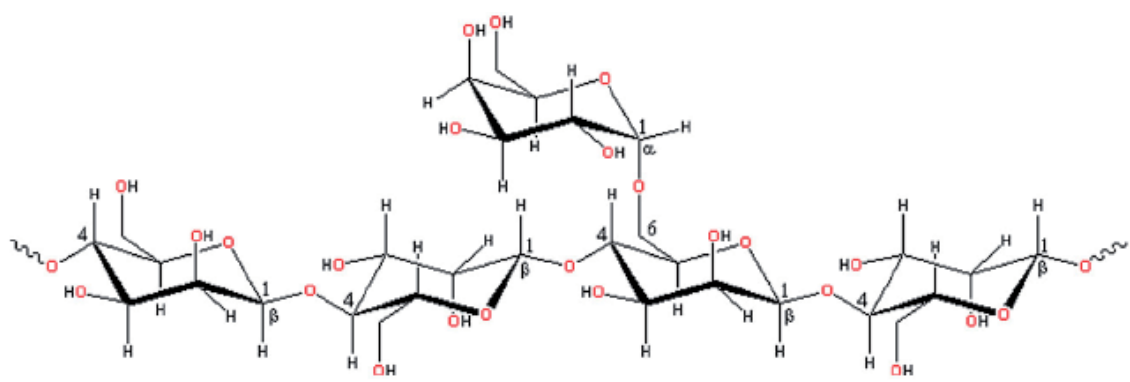

(a)
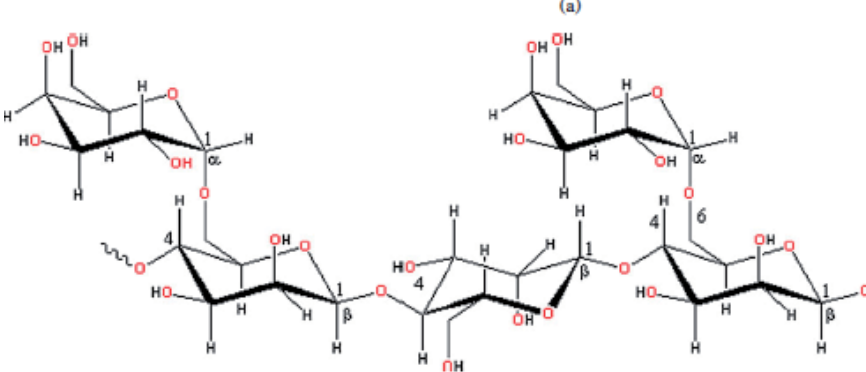

(b)

Figure 4.

Molecular structure of LBG (a) and GG (b) from [14]. 


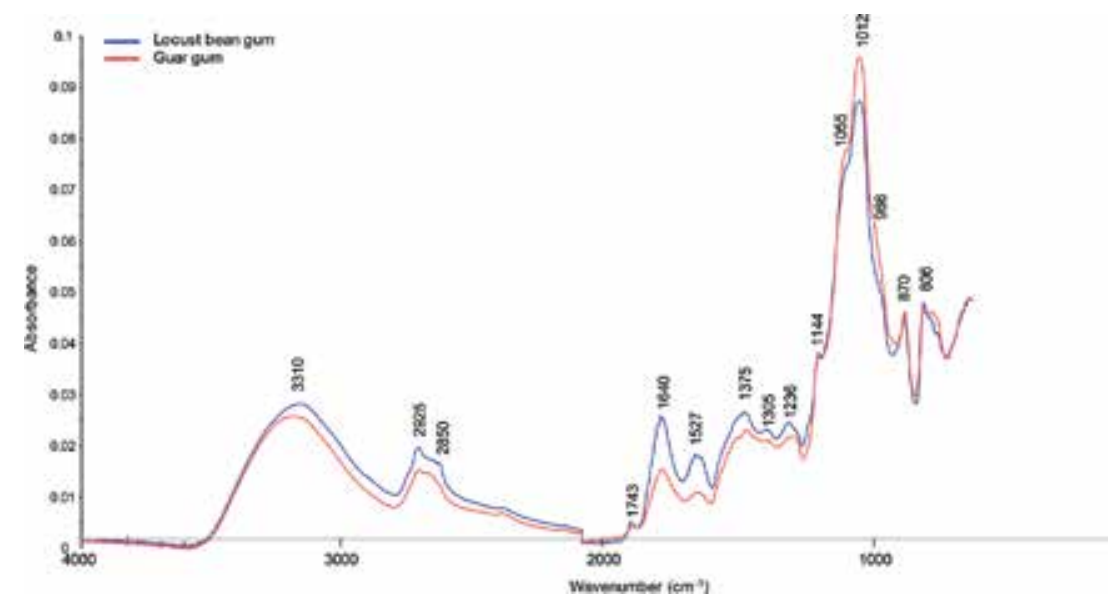

Figure 5.

FTIR-ATR spectra of $L B G$ and $G G$.

globulin, and glutelin [2]) could be found in LBG because of a greater contamination by germ particles; lipids were also detected, just like the presence of rhamnose, arabinose, xylose, and glucose, contaminants proceeding from the seed coat [23].

The broadband between 3700 and $3000 \mathrm{~cm}^{-1}$ describes the $\mathrm{O}-\mathrm{H}$ and $\mathrm{N}-\mathrm{H}$ stretching vibration (hydrogen bonding) attributed to water, amide, and carbohydrates. The spectral zone between 3000 and $2800 \mathrm{~cm}^{-1}$ contains two bands assigned to $\mathrm{C}-\mathrm{H}$ stretching vibration in methylene groups of rings $\left(\nu_{\mathrm{as}} \mathrm{CH}_{2}\right.$ at $2925 \mathrm{~cm}^{-1}$ and $\nu_{\mathrm{s}} \mathrm{CH}_{2}$ at $\left.2871 \mathrm{~cm}^{-1}\right)$. The carbonyl signal at $1743 \mathrm{~cm}^{-1}(\mathrm{C}=\mathrm{O}$ stretching vibration) could be in relation with the amide group of amino acids. A spectrum of GG reference material does not reveal the bands of these contaminants [10]. The band pointed at $1640 \mathrm{~cm}^{-1}$ is due to the presence of bound water $(\mathrm{O}-\mathrm{H}$ bending of absorbed water), which cannot be eliminated despite the freeze-drying. This last band could also be attributed to the axial deformation of $\mathrm{C}=\mathrm{O}$ bond (amide band I) and the one pointed at $1527 \mathrm{~cm}^{-1}$ could correspond to the angular deformation of $\mathrm{N}-\mathrm{H}$ bond (amide band II) or to amine $\mathrm{N}-\mathrm{H}$ deformation vibrations due to the presence of impurities such as proteins and amino acids found in the germ and seed coat, badly remaining during the purification process [24].

The peaks observed in the spectra between 1480 and $1190 \mathrm{~cm}^{-1}$ represented $\mathrm{C}-\mathrm{H}, \mathrm{C}-\mathrm{OH}\left(1236 \mathrm{~cm}^{-1}\right)$, and $\mathrm{H}-\mathrm{C}-\mathrm{H}$ deformation vibration (bending).

Depending on the level of protein impurities, a band at $1236 \mathrm{~cm}^{-1}$ could be assigned to the amide band III $(\mathrm{C}-\mathrm{N})$ vibration mode [24]. The following spectral region (1190-900 $\mathrm{cm}^{-1}$ ) presents different bands contributing to the skeletal vibrations and glycosidic bonds $(\nu \mathrm{CC}, \nu \mathrm{COC}, \nu \mathrm{CCO}$, and $\delta \mathrm{OCH})$ of galactomannans' sugar composition. The $\mathrm{C}-\mathrm{O}$ stretching mode of pyranose ring exists as a small shoulder at $1055 \mathrm{~cm}^{-1}$ and a broad peak at $1012 \mathrm{~cm}^{-1}$, while the shoulder at $966 \mathrm{~cm}^{-1}$ is a characteristic contribution of $\mathrm{C}-\mathrm{OH}$ bending [25]. At lower wavenumbers $\left(900-700 \mathrm{~cm}^{-1}\right)$, peaks at 806 and $870 \mathrm{~cm}^{-1}$ appear that are related with anomeric $\mathrm{C}-\mathrm{H}$ deformation bands $(\mathrm{CCH}$ and $\mathrm{OCH})$ of structural isomers ( $\alpha$ or $\beta$-pyranose compounds), equatorial $\mathrm{C}-\mathrm{H}$ deformation bands (nonglycosidic), and skeletal symmetric and asymmetric ring vibrations (CCO, COC, and OCO) $[26,27]$.

The FTIR-ATR spectrum of each sugar is presented in Figure 6.

These compounds have a $\mathrm{CH}_{2} \mathrm{OH}$ labile group capable of generating intra- and intermolecular hydrogen bonding. This $\mathrm{CH}_{2} \mathrm{OH}$ labile group and the arrangement of the other $\mathrm{OH}$ group on pyranose ring affect differently the spectral region between 3500 and $3000 \mathrm{~cm}^{-1}$. The reversed hydroxyl group orientation in the C-2 and C-4 atom in the 


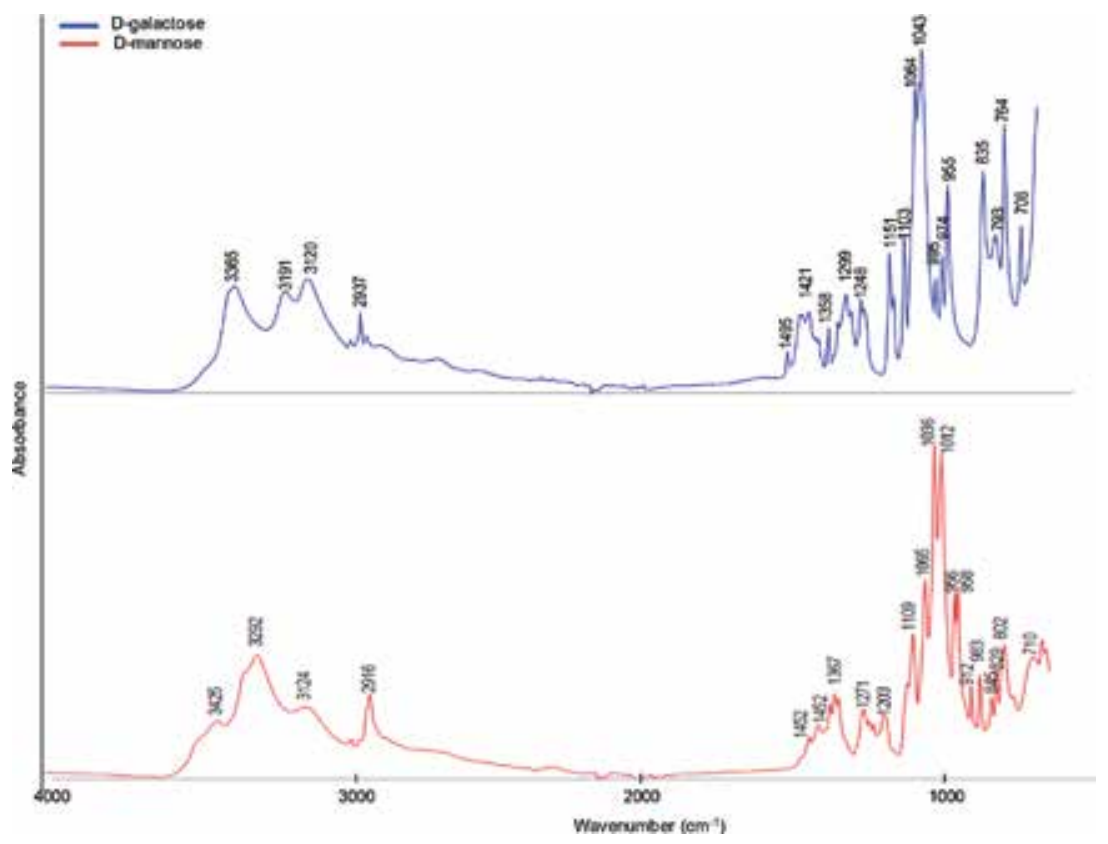

Figure 6.

FTIR-ATR spectra of D-mannose and D-galactose.

structure results in differences in positions and intensities of several bands in the region of $\mathrm{CH}_{2}$ scissoring (1495-1420 cm $\mathrm{cm}^{-1}$ ), between 1400 and 1100 where bands of $\mathrm{CH}_{2}$ wagging and twisting, $\mathrm{C}-\mathrm{O}$ and $\mathrm{C}-\mathrm{C}$ stretching vibration of backbone, and $\mathrm{COH}$ bending of primary and secondary bonded alcohol appear. Because of the equatorial and axial $\mathrm{OH}$ position, the glycosidic and anomeric regions $\left(1000-700 \mathrm{~cm}^{-1}\right)$ show also spectral differences about skeletal stretching $(\mathrm{CO}, \mathrm{CC}$, and $\mathrm{COC})$ vibration coupled with $(\mathrm{COH}$, $\mathrm{CCH}$, and CCO) deformation bands (Table 2) [27, 28] .

\subsection{PCA of gum FTIR-ATR spectra}

PCA, carried out on all of the normalized infrared spectra of gums, shows that on the components 1 and 2, which represent respectively 57 and $24 \%$ of the total spectral variance, the samples of GG in red and LBG in blue form two groups perfectly separated from each (Figure 7). The best separation between clusters was obtained with a selection of variables in anomeric region, between 1450 and $700 \mathrm{~cm}^{-1}$.

The mid-infrared technique allows characterizing the commercial samples of GG and LBG. Globally, all GG samples are negatively projected; LBG samples are positively projected along PC1, while PC2 is a representative of the intragroup variability. The spectral band characteristics of GG and LBG powders are observable on PC1 loading (Figure 8).

The negative part of PC1 loading characterize more LBG samples by exalting spectral bands pointed at 1386,1315 , and $1236 \mathrm{~cm}^{-1}$ (amide bands) characterizing the proteins whose content appears more important in the case of LBG. The band at 1170 could be attributed to $\mathrm{C}-\mathrm{O}$ vibrations of locust bean galactomannan. The large dispersion of locust bean samples along PC1 could be due to the presence of impurities in gum powders coming from husk and germ, (like insoluble matter, proteins, amino acid, etc.), residual compounds resulting from the various steps of extraction, and purification processes. Different maturity stages of seed, geographic origin, and climatic conditions could also be responsible for the variability of the chemical composition of these galactomannan gums. The positive part of PC1 reveals 


\begin{tabular}{|c|c|c|c|}
\hline \multirow{2}{*}{$\begin{array}{l}\text { Spectral } \\
\text { region }\end{array}$} & \multicolumn{2}{|c|}{ Wavelength $\left(\mathrm{cm}^{-1}\right)$} & \multirow[t]{2}{*}{ Chemical group assignment } \\
\hline & D-Galactose & D-Mannose & \\
\hline $3500-3000$ & $\begin{array}{l}3365,3191 \\
\quad 3120\end{array}$ & 3425 & $\mathrm{O}-\mathrm{H}$ stretching vibration, hydrogen bonded \\
\hline $3000-2800$ & 2937 & 2916 & $\mathrm{C}-\mathrm{H}$ stretching vibration of $\mathrm{CH}_{2}$ group \\
\hline $1500-1200$ & $\begin{array}{l}1495,1421 \\
1358,1299 \\
1248\end{array}$ & $\begin{array}{c}1452,1419,1367 \\
1271,1203\end{array}$ & $\mathrm{CH}_{2}$ symmetric deformation, $\mathrm{C}-\mathrm{OH}$ deformation \\
\hline $1200-950$ & $\begin{array}{l}1151,1103 \\
1064,1043 \\
995,974\end{array}$ & $\begin{array}{l}1109,1065,1036 \\
1012,956,958 \\
912\end{array}$ & $\begin{array}{c}\mathrm{C}-\mathrm{C} \text { skeletal, } \mathrm{C}-\mathrm{O}-\mathrm{C}, \mathrm{CO}-\mathrm{O}-\mathrm{C}, \mathrm{C}-\mathrm{O} \\
\text { stretching vibration, } \mathrm{C}-\mathrm{O} \text { bending of } \mathrm{C}-\mathrm{OH} \\
\text { group, } \mathrm{OCH} \text { bending }\end{array}$ \\
\hline $950-700$ & $\begin{array}{c}835,793,764 \\
706\end{array}$ & $845,829,802$ & $\begin{array}{c}\text { Anomeric } \mathrm{C}-\mathrm{H} \text { deformation ( } \alpha \text { or } \beta \text { ), equatorial } \\
\mathrm{C}-\mathrm{H} \text { deformation (nonglycosidic) and } \\
\text { asymmetric and symmetric ring vibration }\end{array}$ \\
\hline
\end{tabular}

Table 2.

Spectral interpretations of pure sugars.

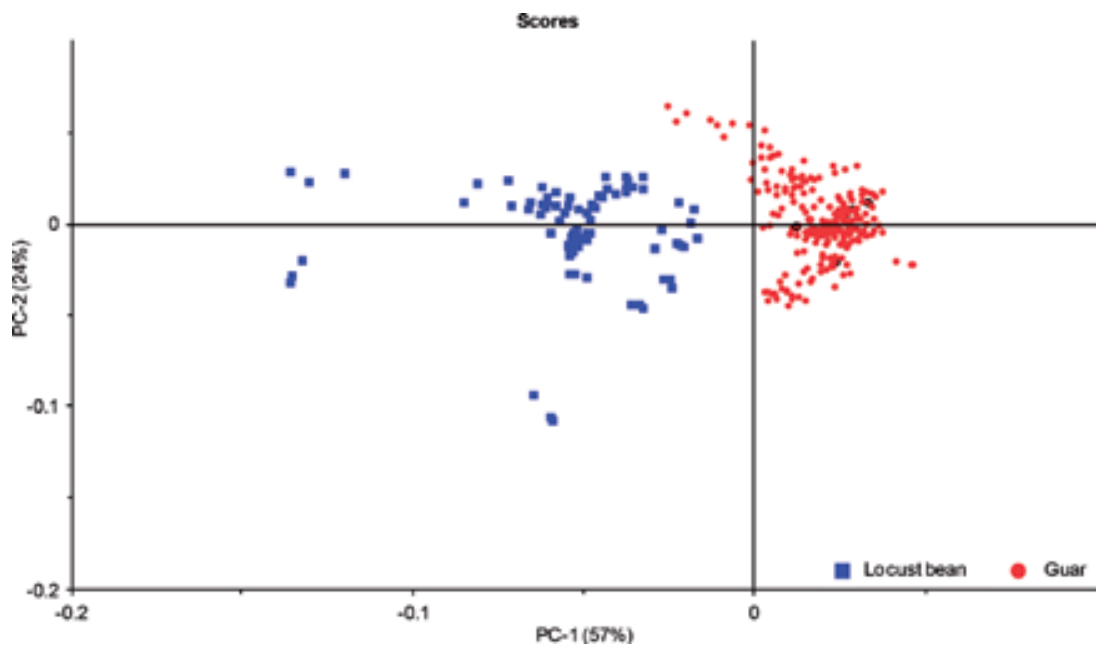

Figure 7.

Representation of GG and LBG samples in (PC1 and PC2) plan (normalized spectral data).

the most intense spectral bands in GG samples at 1066, 1012, 964, 863, 819, and $771 \mathrm{~cm}^{-1}$. Bands at 1012 and $964 \mathrm{~cm}^{-1}$ are attributed to C-O-H and C-O vibrations, respectively. The band at $771 \mathrm{~cm}^{-1}$ is due to ring stretching and ring deformation of $\beta$-D-(1-4) and $\alpha$-D-(1-6) linkages. These last ones are specific to the anomeric region where $\mathrm{C}-\mathrm{O}$ stretching bands are more representative because of the largest number of galactose units (1-6) linked to $\beta$-D-mannopyranosyl backbone in GG.

While GG was richer in galactosyl residue, no specific bands of D-galactose were found in positive part of PC1 loading representing the GG samples. The comparison with the spectral bands of pure D-galactose or D-mannose is not a good way because of their crystalline structure (free form) that is not the case in their polymer form. Another explanation could be a possible interference with the presence of water that modifies the band's resolution in the anomeric region as it is observable in Figure 9 presenting sugar profiles under crystalline and hydrated forms. 
Discrimination by Infrared Spectroscopy: Application to Micronized Locust Bean and Guar Gums DOI: $h$ ttp://dx.doi.org/10.5772/intechopen.87568

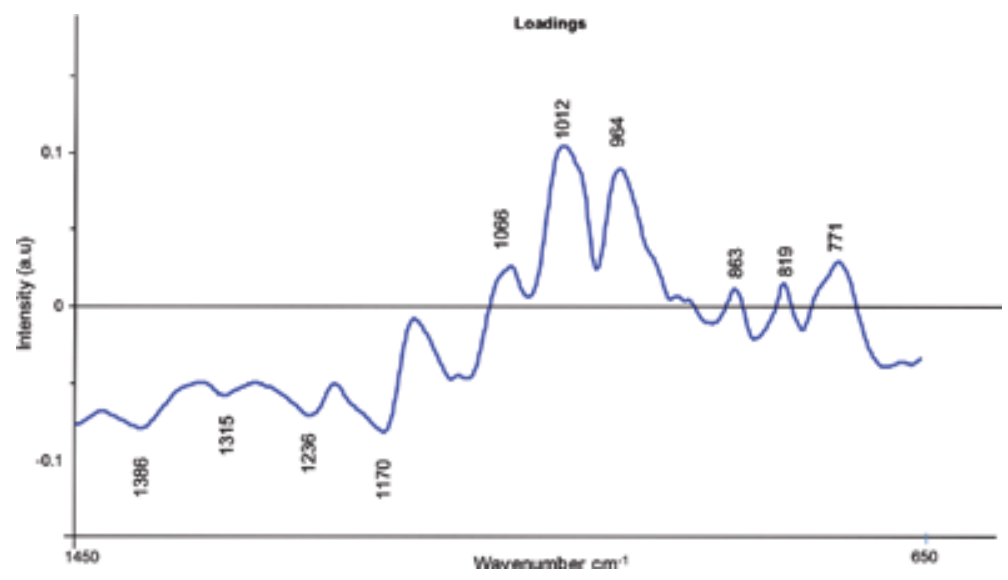

Figure 8.

PC1 loading.
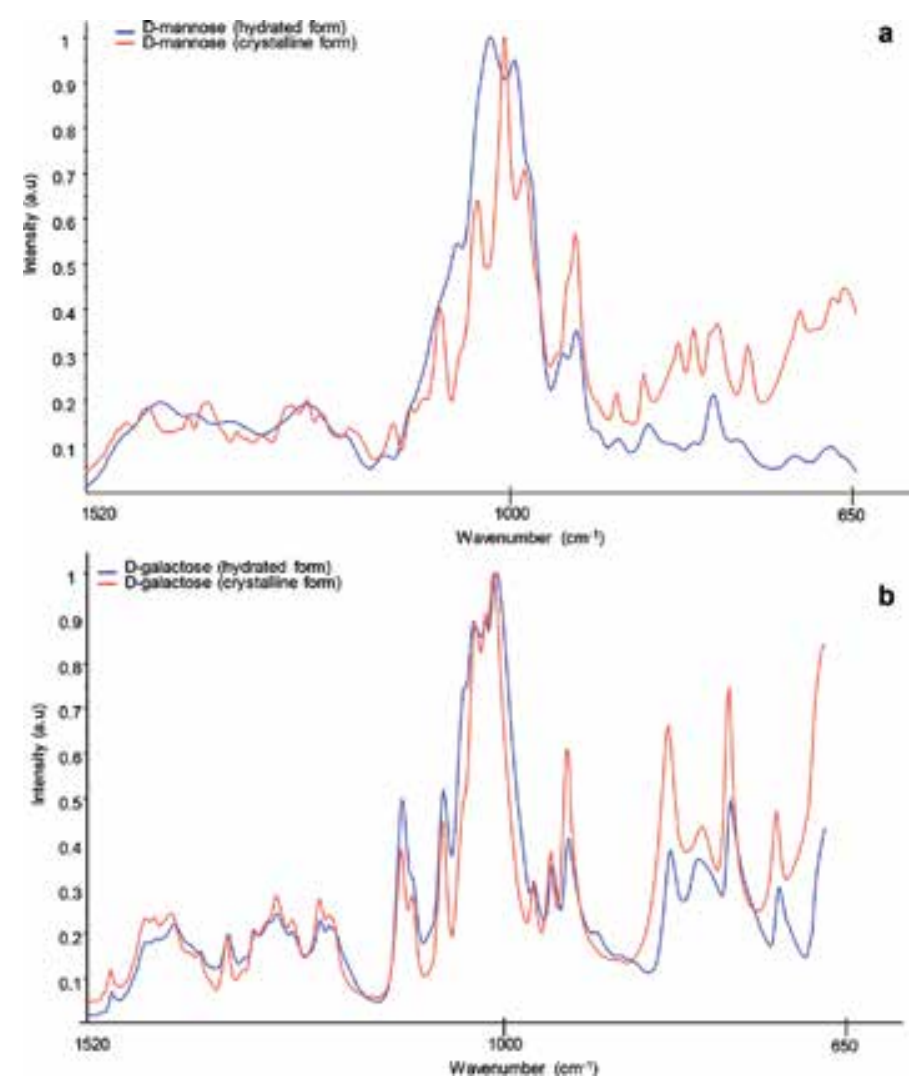

Figure 9.

Spectral profiles of D-mannose (a) and D-galactose (b) under crystalline and hydrated forms.

\subsection{Prediction of species origin by PLS-1-DA regression}

The classification into varietal origin (GG or LBG) was performed using PLS-1-DA analysis. The calibration dataset was composed of 37 GG and 13 LBG $(\mathrm{n}=50$ samples $\times 3$ spectra $=150)$. Different samples of calibration set, 12 LBG and 37 GG $(n=49$ samples $\times 3$ spectra $=147)$, have constituted the validation set. 


\begin{tabular}{lc}
\hline Model parameters & Values \\
\hline Spectra number for calibration & 150 \\
\hline Spectra number for validation & 147 \\
\hline LV & 3 \\
\hline SEC & 0.08 \\
\hline SEP & 0.11 \\
\hline $\mathrm{R}^{2}$ & 0.97 \\
\hline $\mathrm{Q}^{2}$ & 0.94 \\
\hline$\%$ Sensibility & 100 \\
\hline$\%$ Specificity & 100 \\
\hline$\%$ Correct classification & 100 \\
\hline SEC or SEP: standard error of calibration or prediction, $R^{2}$ or $Q^{2}$ correlation coefficients for calibration or prediction. \\
\hline
\end{tabular}

Table 3.

$P L S-1-D A$ parameters for $L B G$.

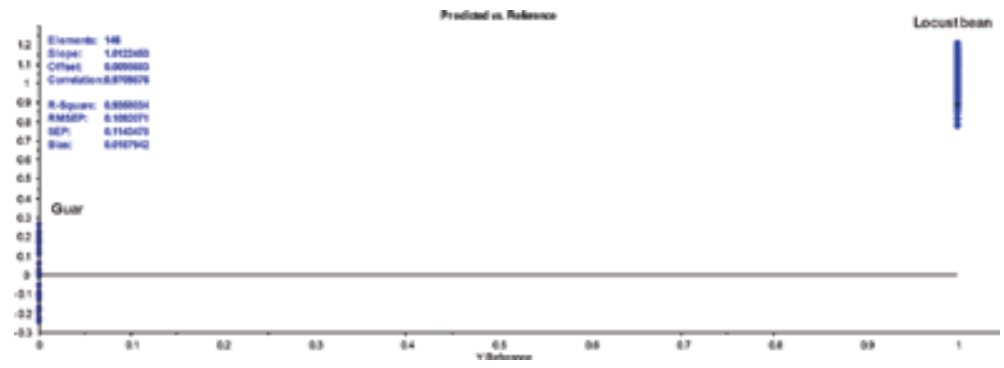

Figure 10.

Predicted versus reference value of gum classification (validation step).

The calibration model had very good quality parameters, as shown in Table 3 , and very good validation results for LBG were obtained using normalized spectra with a selection of wavelength region from 1450 to $700 \mathrm{~cm}^{-1}$.

For GG validation, the different statistic parameters were also closed to $100 \%$.

The following graph (Figure 10) shows that predicted values from validation data are closed to zero for GG and 1 for LBG.

Calibration model has satisfying quality parameters, as shown in Figure 10, with RMSEP ranging from 0.11 for LBG to 0.94 for $\mathrm{Q}^{2}$ (R-square), and $100 \%$ good classification is obtained. The predicted species origins are never given by zero or one results because the different rates of carbohydrates in the samples vary according to the origins. As a matter of fact, there is a natural variation of the carbohydrate rates that can notably be a function of geographic origins and harvest dates.

Contrary to the results of Prado et al. [12] who published that diffuse reflectance (DRIFT) method was better suited for differentiation of gum type, ATR technique showed here a very good classification. It was noted that these authors have realized spectra with a ZnSe multiple bounce ATR on gum aqueous solutions, heated for their preparation. Nevertheless, the work of Wang et al. [14] showed that the computer-simulated molecular space filling structure of GG and LBG was different in no solvent and aqueous environments. In the aqueous environment, GG form presented a more complicated structure than LBG form because of the increase in galactose units on the mannose backbone [14]. In conclusion, spectral data in solid or liquid environment were difficult to compare as done by Prado et al. [12] because the intermolecular interactions in the structure of gums were not the same. 


\subsection{Quantitative analysis of gum mixtures}

As PLS-DA allowed easily the prediction of the botanical origin of galactomannans, LDA was adapted to the prediction of the proportions (or weights, $\mathrm{w}_{\mathrm{i}}$ ) of pure compounds in blends with the advantage to be governed by a constant sum of $\mathrm{w}_{\mathrm{j}}$ (equal to $100 \%$ ). But the fact to provide ordinal predictions (or class) leads to a strict response of model, which considers a wrong classification even if the predictive weights are slightly different from reference data. A selection of variables between 1900 and $650 \mathrm{~cm}^{-1}$ and an average reduction (by two) of variables in this spectral zone were performed to make simplex iterative operations possible, and because in LDA classification method, the number of objects (or pairs of weights) should be larger than the number of variables (wavenumbers). In this way, 325 variables, 11 pairs of weights between 0 and 1 (with a constant increment of 0.1 ), and a value of $k$ (number of iterations in Scheffé's simplex) equal to 400 to be superior at the variable number (the constraint of LDA calibration step) were used to generate 4400 artificial bends $(11 \times 400)$ from simplex design.

An example of the repartition of simulated blends generated with this method is given in Figure 11 where a step of $10 \%$ was chosen to clarify the graphic representation. The graph has been obtained after realizing a PCA on blends' binary data.

Dispersed experimental samples were placed at the extrema of PC1 axis: LBG at the left in the negative part of PC1 and GG at the right in the positive part, respectively. It well appears that simulated blends well take into account the intrinsic variability of pure components, but in certain regions, an inevitable overlapping originating from the high variability of chemical composition of pure gums is also observed.

Five different LDA calibration models were built with five values of weight step $(0.100,0.050,0.04,0.02$, and 0.007$)$. The robustness of each calibration model has been tested with four validation sets obtained with steps of $0.10,0.083,0.067$, and 0.002 , without constraint about the number of blends. The results are resumed in Table 4.

All weights of GG and LBG in pure state and mixtures containing 2-10\% of GG were well predicted in the validation step. A percentage of $61 \%$ was obtained when the increment chosen in validation was lower than the calibration step.

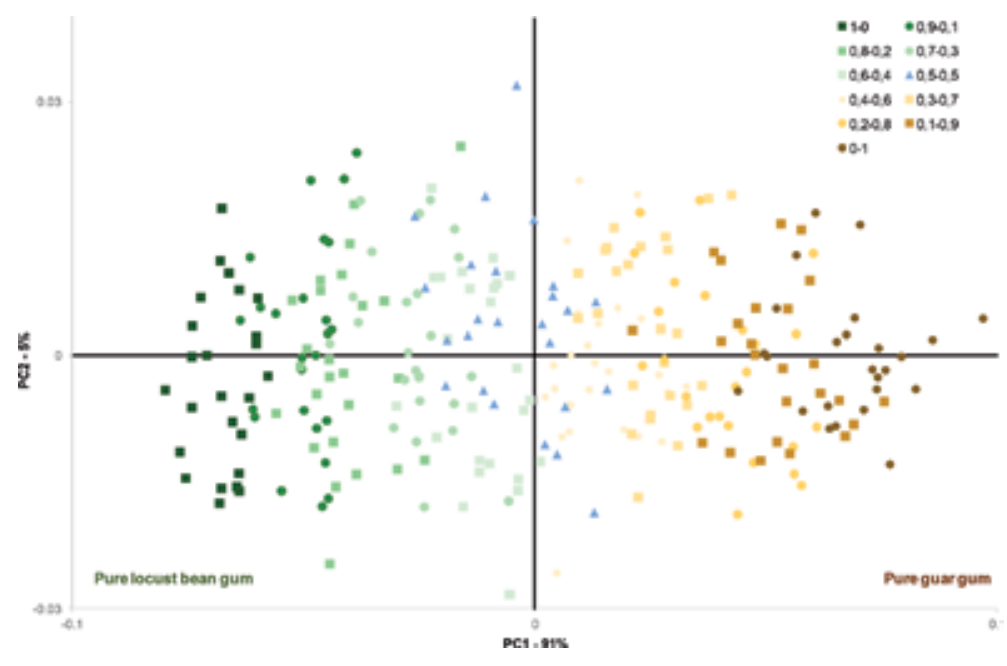

Figure 11.

Location of the binary blends in the simplex space defined by GG and LBG proportions (step of $10 \%$ ). 


\begin{tabular}{lcccccc}
\hline & \multicolumn{5}{c}{ Calibration model } \\
\hline Blends number & & 4400 & 8400 & 10400 & 20400 & 60400 \\
\hline \multirow{2}{*}{1100} & Weight step & 0.100 & 0.050 & 0.040 & 0.020 & 0.0067 \\
\hline 780 & 0.100 & $100 \%$ & $100 \%$ & $100 \%$ & $100 \%$ & $100 \%$ \\
\hline 1200 & 0.083 & $100 \%$ & $100 \%$ & $100 \%$ & $100 \%$ & $100 \%$ \\
\hline 5100 & 0.067 & $100 \%$ & $100 \%$ & $100 \%$ & $100 \%$ & $100 \%$ \\
\hline
\end{tabular}

Table 4.

Prediction of correct weight for different validation sets.

\section{Conclusions and outlook}

Guar and locust bean gums are galactomannans with similar chemical structure because only the galactose/mannose ratio differentiates them. Depending on the geographic origins of seeds and their industrial manufacturing process, this ratio can be a variable and improves certain variability in their chemical composition, weakly discernible according to the analytical techniques used to characterize these gums. Despite the similarity of the infrared spectral signatures of guar and locust bean gums and the overlapping of IR bands due to inter- and intramolecular interactions, this work showed the feasibility of using FTIR-ATR technique to discriminate GG and LBG with the help of chemometric treatments. Best results have been obtained with a variable selection in the anomeric spectral region (between 1450 and $650 \mathrm{~cm}^{-1}$ ) to differentiate gum samples using a principal component analysis and to predict the species origin with a partial least square regression. A particular approach has been proposed to quantify the proportion of gums in blends with a good accuracy with the advantage to detect an adulteration of LBG by GG from their spectral profiles. The Scheffés simplex approach allowed taking into account the variability of the chemical composition of gum samples due to different environmental parameters and manufacturing processes and generating a lot of simulated blends to increase the robustness of LDA calibration model. The approach with computational blends is a rapid and a low cost way to generate an FTIR-ATR dataset used in favor of quality control and prediction of adulteration. 


\section{Author details}

Catherine Rébufa, Fabien Girard, Jacques Artaud and Nathalie Dupuy* Aix Marseille Univ, Avignon Université, CNRS, IRD, IMBE, Marseille, France

*Address all correspondence to: nathalie.dupuy@univ-amu.fr

\section{IntechOpen}

(C) 2019 The Author(s). Licensee IntechOpen. This chapter is distributed under the terms of the Creative Commons Attribution License (http://creativecommons.org/licenses/ by/3.0), which permits unrestricted use, distribution, and reproduction in any medium, provided the original work is properly cited. (cc) BY 


\section{References}

[1] Aqualon. Guar and Guar Derivatives Oil and Gas Field Applications. 2007. Available from: http://www.ashland. com/Ashland/Static/Documents/AAFI/ PRO_250-61_Guar.pdf

[2] Barak S, Mudgil D. Locust bean gum: Processing, properties and food applications-A review. International Journal of Biological Macromolecules. 2014;66:74-80

[3] Thombare N, Jha U, Mishra S, Siddiqui MZ. Guar gum as a promising starting material for diverse applications: A review. International Journal of Biological Macromolecules. 2016;88:361-372

[4] Hasan AMA, Abdel-Raouf ME. Applications of guar gum and its derivatives in petroleum industry: A review. Egyptian Journal of Petroleum. 2018;27:1043-1050

[5] Gupta S, Variyar PS. Chapter 12: Guar gum: A versatile polymer for the food industry. In: Grumezescu AM, Holban AM, editors. Handbook of Food Bioengineering. Vol. XX: Biopolymers for Food Design. Cambridge: Elsevier, Academic Press; 2008. pp. 383-407

[6] Gillet S, Simon M, Paquot M, Richel A. Synthèse bibliographique de l'influence du procédé d'extraction et de purification sur les caractéristiques et propriétés d'une gomme de caroube. Biotechnology, Agronomy, Society and Environment. 2014;18:97-107

[7] Srečec S, Dunkić V, Bezić N, Kremer D, Erhatić R. Some doubts controversies about anatomy of locust bean (Ceratonia siliqua L.) seed coat. Genetics, plant breeding and seed production. In: Proceeding of 53rd Croatian \& 13th International Symposium on Agriculture; 18-23 February 2018; Vodice, Croatia. 2018
[8] Vishwakarma RK, Shivhare US, Nanda SK. Novel method and machine for dehulling of guar seeds and optimisation of dehulling process. Food and Bioproducts Processing. 2016;99:51-57

[9] Pitkänen L, Tuomainen P, Mikkonen $\mathrm{K}$, Tenkanen $\mathrm{M}$. The effect of galactose side units and manna chain length on the macromolecular characteristics of galactomannans. Carbohydrate Polymers. 2011;86:130-1235

[10] Liyanage S, Abidi N, Aud D, Moussa H. Chemical and physical characterization of galactomannan of extracted from guar cultivars (Cyamopsis tetragonolobus L.). Industrial Crops and Products. 2015;74:388-396

[11] Prajapati VD, Girish KJ, Moradiya NG, Randeria NP, Nagar BJ, Naikwadi NN, et al. Galactomannan: A versatile biodegradable seed polysaccharide. International Journal of Biological Macromolecules. 2013;60:83-92

[12] Prado BM, Kim S, Ozen BF, Mauer LJ. Differenciation of carbohydrate gums and mixtures using Fourier transform infrared spectroscopy and chemometrics. Journal of Agricultural and Food Chemistry. 2005;53:2823-2829

[13] Ćopíková J, Synystsya A, Ćerná M, Kaasová J, Novotná M. Application of FT-IR spectroscopy in detection of food hydrocolloids in confectionery jellies and food supplements. Czech Journal of Food Sciences. 2001;19:31-36

[14] Wang J, Somasundaram P. Study of galactomannose interaction with solid using AFM, IR and allied techniques. Journal of Colloid and Interface Science. 2007;309:373-338

[15] Mudgil D, Barak S, Khatkae BS. $\mathrm{X}$-ray diffraction, IR spectroscopy and 
thermal characterization of partially hydrolysed guar gum. International Journal of Biological Macromolecules. 2012;50:1035-1039

[16] Yen SN, Choi SM, Phillips DL, Ma CY. Raman and FTIR spectroscopic study of carboxymethylated non-starch polysaccharides. Food Chemistry. 2009;114:1091-1098

[17] Alabdi F, Elharfi N, Balouki A, Kzaiber F, Oussama A. Locust bean origin classification by FTIR spectroscopy and chemometrics. Journal of Chemistry and Chemical Engineering. 2001;5:1020-1029

[18] Lafhal S, Vanloot P, Bombarda I, Valls R, Kister J, Dupuy N. Raman spectroscopy for identification and quantification analysis of essential oil varieties: A multivariate approach applied to lavender and lavandin essential oils. Journal of Raman Spectroscopy. 2015;46:577-585

[19] Bombarda I, Dupuy N, Le Van Da JP, Gaydou EM. Comparative chemometric analyses of geographic origins and compositions of lavandin var. Grosso essential oils by mid-infrared spectroscopy and gas chromatography. Analytica Chimica Acta. 2008;613:31-39

[20] Semmar N, Artaud A. A new simplex-based approach predicting olive oil blend compositions from fatty acid data. Journal of Food Composition and Analysis. 2015;43:149-159

[21] Scheffé H. Experiments with mixtures. Journal of the Royal Statistical Society: Series B. 1958;20:344-360

[22] Scheffé H. Simplex centroid designs for experiments with mixtures. Journal of the Royal Statistical Society: Series B. 1963;25:235-263

[23] Dakia A, Blecker C, Robert C, Wathelet B, Paquot M. Composition and physicochemical properties of locust bean gum extracted from whole seeds by acid or water dehulling pre-treatment. Food Hydrocolloids. 2008;22:807-818

[24] Figueiró SD, Góes J, Moreira RA, Sombra ASB. On the physicochemical and dielectric properties of glutaraldehyde crosslinked galactomannan-collagen films. Carbohydrate Polymers. 2004;56:313-320

[25] Cerquiera MA, Souza BWS, Simões J, Teixeira JA, Domingues MRM, Coimbra MA, et al. Structural and thermal characterization of galactomannans from non-conventional sources. Carbohydrate Polymers. 2011;83:179-185

[26] Talari ACS, Martinez MAG, Movasaghi Z, Rehman S, Rehman IU. Advances in Fourier transform infrared (FTIR) spectroscopy of biological tissues. Applied Spectroscopy. 2017;52:456-506

[27] Socrates G. Infrared and Raman Characteristic Group Frequencies Tables and Charts. 3rd ed. New York: John Wiley \& Sons, Ltd; 2001. p. 347

[28] Wiercigroch E, Szafraniec E, Czamara K, Pacia MZ, Majzner K, Kochan K, et al. Raman and infrared spectroscopy of carbohydrates: A review. Spectrochimica Acta, Part A: Molecular and Biomolecular Spectroscopy. 2017;185:317-335 



\title{
Raman Spectroscopy and Imaging of Carbon Allotropes
}

\author{
Gustavo Morari do Nascimento
}

\begin{abstract}
Raman spectroscopy is employed to study a myriad of complex materials due to the coupling of different resonances process and microscopic resources. For instance, vibrational and electronic aspects of carbon allotropes can be deeply investigated by resonance Raman spectroscopy. By selecting the appropriate laser line, it is possible to monitor different aspects of the samples, such as the behavior of metallic or semiconducting tubes and the graphene with different layers or chemical modifications. In this chapter, the potentialities of Raman technique will be exemplified in some examples obtained in our group about the characterization of carbon nanotubes and graphene. For instance, the doping process of nanotubes, carbon tube interactions with molecular magnets, and inhomogeneity of graphene samples will be discussed.
\end{abstract}

Keywords: Raman spectroscopy, Raman imaging, carbon nanotubes, graphene, modification

\section{Introduction}

\subsection{Nanostructures}

In the recent years, the synthesis and characterization of nanomaterials have been one of the most efficacious ways to produce new materials with improved or completely new properties [1]. Their physical dimensions can be used to classify the nanomaterials in subgroups. One-dimensional (1D) nanostructures are systems in which one of the spatial dimensions has less than $100 \mathrm{~nm}$, such as carbon nanotubes, metallic nanowires, or zeolites having 1D cavities (Figure 1). Lamellar materials are classified as two-dimensional (2D) nanostructures, because there are formed by platelets piled up in one crystallographic direction, as the graphite and clays. For materials having nanocavities or structures those follow in all directions, they are named as three-dimensional nanostructures (3D; as some zeolites). When the material is symmetric in all directions, it is considered as zero-dimensional (0D) nanostructures, as found in quantum dots, fullerenes, or cyclodextrins (Figure 1).

Nanostructures are systems in which at least one of the spatial dimensions is smaller than $100 \mathrm{~nm}$ [1]. The synthesis of controlled dimensional nanostructures and the characterization of the intrinsic and potentially peculiar properties of these nanostructures are central themes in nanoscience. The study of different nanostructures has great potential to test and understand fundamental concepts about the role of particle dimensionality on their physicochemical properties. Among the various materials studied in the literature, undoubtedly, 
3-D

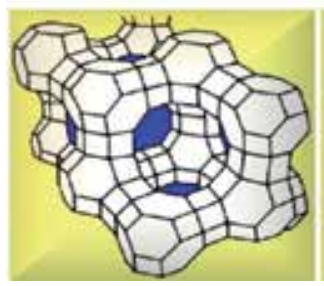

2-D

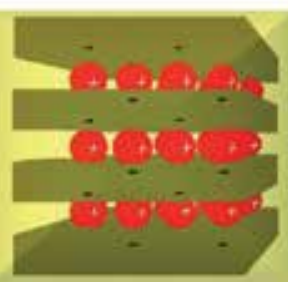

1-D

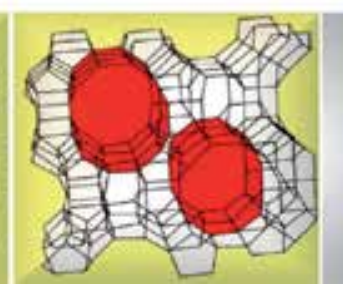

0-D

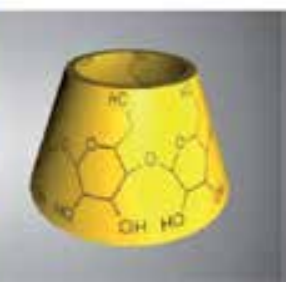

Figure 1.

Schematic models for $3 D, 2 D, 1 D$, and $o D$ materials.

carbon-derived materials, especially fullerenes and nanotubes, and more recently graphenes, are of particular note.

There are two central questions for the study of chemistry and physics of these nanostructures: (i) how controlled dimensionality nanostructures can be fabricated, and (ii) what are the intrinsic and potentially peculiar properties of these nanostructures. ID structures have great potential for testing and understanding fundamental concepts about the role of dimensionality and size over the properties. For example, 1D systems must have singularities in their electronic density of states. There are also several applications, as 1D systems are smaller structures that can be used for efficient electrical transport. Their selected properties can be explored, for example, in nanoelectronics. Among the carbon nanostructures, we highlight carbon nanotubes and graphenes [2-4].

\subsection{Carbon allotropes}

The discovery of fullerenes in 1985 opened a new field in chemistry [5]. Since this discovery, research with carbon structures has grown rapidly. In 1991, Sumio Iijima was the first researcher to observe some unusual carbon structures under a transmission electron microscope. Iijima called these structures as carbon nanotubes (CNTs) [2], because they consisted of many cylindrical coiled carbon layers. The layers have carbon atoms attached by six-membered rings and are stacked in the c-crystallographic direction, such as in graphite. CNTs can be open-ended or closed-ended with closed ends having five-membered carbon rings as in fullerenes.

The CNTs can be multiwalled carbon nanotubes (MWCNTs), double-walled carbon nanotubes (DWCNTs), or single-walled carbon nanotubes (SWCNTs). In fact, depending on the way, the graphite layers are coiled, i.e., the different combinations of the vectors (defined by the integers $n, m$ ) that define each tube geometrically, they can be classified as “armchair," “zigzag," and "chiral” (Figure 2). As consequence of this peculiar geometry, the electronic conductivity and optical properties, such as luminescence and light scattering, are dependent of the tube geometry [6-8].

CNTs attract great attention because they are model systems for nanoscience and have great potential for applications such as composite materials, batteries, sensors, and nanoscale electronics. Interest in CNTs is in their unique structure and properties, their small size (from 0.8 to $2 \mathrm{~nm}$ in diameter, see Figure 1), their ability to be metallic or semiconductor depending on their geometric structure (Figure 2), their exceptional ballistic transport properties and thermal conductivity, optical polarizability, and high structural perfection $[6,7]$.

SWCNTs have a relatively simple structure, allowing detailed calculations of their electronic structure [7, 9-12]. The unique optical properties of CNTs are due to the confinement of electronic states in one direction, resulting in the so-called 


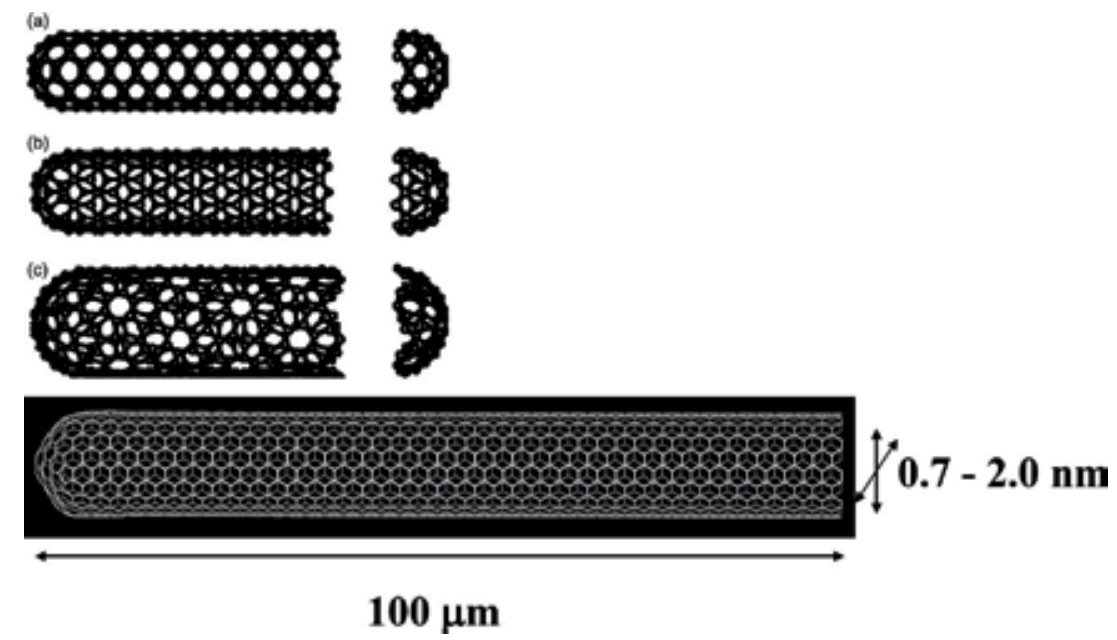

Figure 2.

Schematic models for single-axis carbon nanotubes with normal axis in (a) direction $\theta=30^{\circ}$ [tube seat $(n, n)$ ], (b) direction $\theta=0^{\circ}$ [zigzag tube $\left.(n, o)\right]$, and $(c)$ direction $0^{\circ}<\theta<30^{\circ}$ [chiral tube $\left.(n, m)\right]$. The values of $m$ and $n$ specify a symmetry and nanotube diameter [6].

van Hove singularities $[10,13]$. The presence of a large number of electron states with very close values in energy leads to an intensification of corresponding photophysical processes, and it is possible through electron absorption, photoluminescence, resonance Raman, and photoelectron spectroscopy techniques to obtain detailed information about the electronic structure and vibration analysis of nanotubes $[7,14,15]$.

Theoretical predictions about the one-dimensional electronic structure of nanotubes have been experimentally verified. The most conclusive evidence comes from Scanning Tunneling Microscopy/Spectroscopy (STM/STS) studies, which showed atomic resolution images and the corresponding electronic structures for metallic and semiconductor nanotubes, and verified the dependence of electronic properties on diameter and helicity [16]. Therefore, the electronic structure of nanotubes depends only on their symmetry, being quite peculiar to solid-state physics. Specifically, the electronic structure may be metallic or semiconductor, depending on diameter and chirality, although there is no difference in the chemical bonds between carbon atoms in different nanotubes.

Despite the unique properties of CNTs, and these are already produced in macroscopic quantities, allowing the study of their physicochemical properties, they still have low solubility in most solvents, as a consequence of their high aggregation, limiting the possibility of chemical manipulation and technological application. Thus, different approaches have been employed to separate or disaggregate CNTs, such as chemical modifications of nanotubes or through interaction with polymers.

Graphite (3D), which is the most well-known allotropic form of carbon, has layers of several carbon atoms bonded with $\mathrm{sp}^{2}$ hybridization (Figure 3), one carbon atom joining three other atoms forming a planar array of hexagons. The layers remain connected via the interaction of van der Waals forces. These twodimensional sheets have the thickness of a carbon atom, which allows them to have different properties that differ from graphite, such as high electrical, thermal conductivity, and mechanical stiffness [3, 4, 18-22]. These layers have been called graphene (2D), which were discovered by scientists André Geim and Konstantin Novoselov at the end of 2004 at the University of Manchester.

Graphene is the allotropic form of carbon most recently studied, due to its wide application in the scientific environment. This material is obtained via graphite 


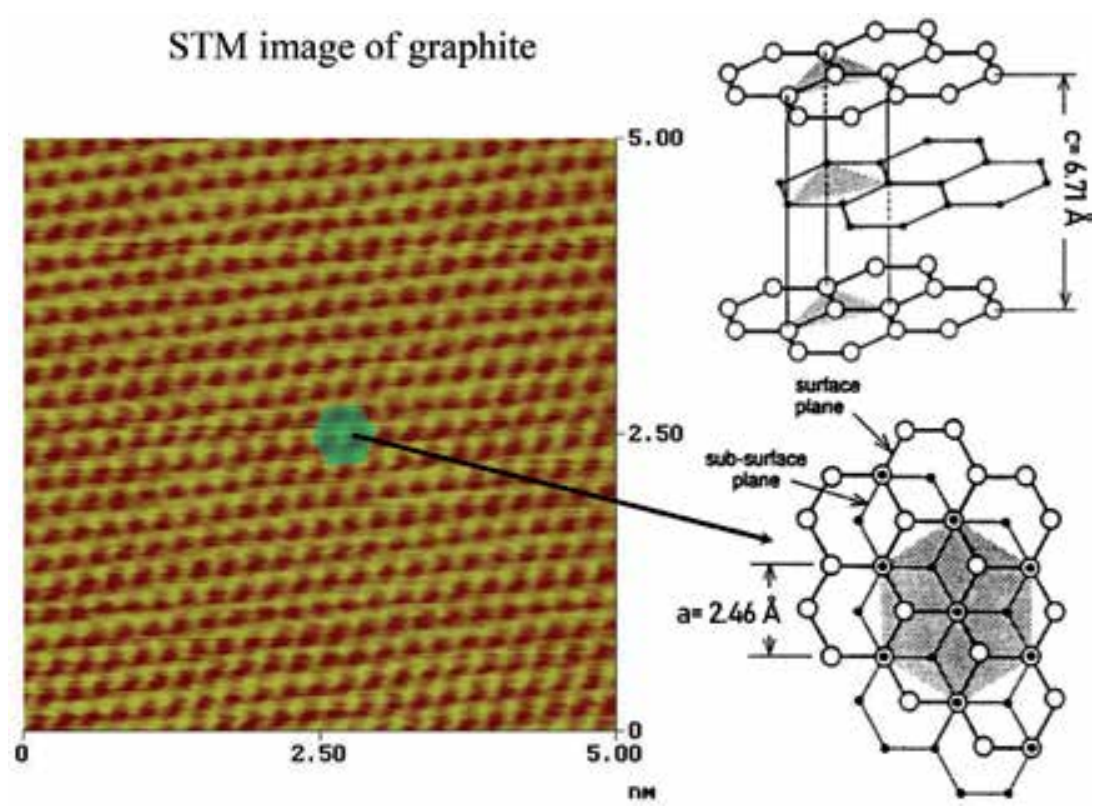

Figure 3.

STM image obtained from a HOPG crystal and the schematic models for the crystalline graphite. The crystallographic parameters were obtained from Ref. [17].

oxidation and exfoliation processes. Depending on the synthesis parameters (oxidant type, reactant proportions, temperature, etc.), graphene sheets or their oxide (GO) or graphene oxide will have distinct properties. Brodie performed the first documented synthesis in 1859 [23]. Since then, several other scientists have prepared graphene oxide (GO) synthesis experiments from graphite $(\mathrm{G})$ in order to reduce the environmental hazards that this synthesis presents in the use of strong oxidants and concentrated acids. Modifications were later made by Staudenmaier (1898), who focused on improving the reaction introduced $\mathrm{H}_{2} \mathrm{SO}_{4}$ to the mixture and some aliquots of solid $\mathrm{KClO}_{3}$ throughout the reaction. With these modifications, the author achieved the synthesis of a more oxidized graphic material and a simplification in the reaction [24]. However, there is release of $\mathrm{ClO}_{2}$ gas, with risks of explosions during the process. In 1958, Hummers proposed some more modifications to this synthesis with the intention of making it safer and more profitable, replacing the oxidizing agent used in the Brodie method with $\mathrm{MnO}_{4}$ and an additive, $\mathrm{NaNO}_{3}$. All the methods mentioned so far make use of strong and toxic reagents for the production of GO, however the use of thermal expansion of graphite, or sonic spacing, those are more green routes, it takes around 6-12 h of preparation.

\subsection{Raman spectroscopy and imaging}

Through the years, the infrared and Raman spectroscopies have been the techniques par excellence for the investigation of the vibrational structure of conjugated materials, such as dyes [25], metallic complexes [26-28], conducting polymers $[29,30]$, nanocomposites [31-33], and carbon allotropes [34-39]. The laser is the common source in Raman spectroscopy, the radiation interacts with the sample through the scattering process. The incident light (laser source) has much more energy than the vibrational levels, however, by the scattering process; the information about the vibrational modes can be accessed. This behavior is very different 
from the IR vibrational spectroscopy, where the radiation energies have the same magnitude of vibrational levels, and by absorption process, the vibrational modes can be studied (Figure 4).

The intensities of the Raman bands are proportional to the fourth power of the scattered frequency $\lambda_{s}^{4}$ when the laser energy is very far from a permitted molecular electronic transition $[40,41]$. This behavior changes dramatically when the laser energy is close to an electronic transition, this condition is known as Resonance Raman. Hence, the intensities of the vibrational modes associated with the excited electronic state in resonance are amplified by $10^{5-7}$ times. For multichromophoric systems, like conducting polymers, it is possible to screen the different chromophores, just by selecting the appropriated laser line energy.

In recent years, the use of Raman spectrometers coupled with different microscopes, from optical to force atomic type, has increased. In Figure 5, the configuration of an optical microscope coupled to a laser source used for Raman measurements is schematically illustrated. The laser lines reach to the sample on the microscope stage by optical elements. The Raman scattered radiation is collected in a scattering angle of $180^{\circ}$ by the same microscope objective and captured by an opening of the spectrometer using a beam splitter. It is necessary that the instrument has a high lighting efficiency, and the collection of scattered radiation must be precisely done, owing to the very small Raman cross section (typically a factor of $10^{-6}$ to $10^{-12}$ of the incident radiation) and the small volume of the sample. Raman microscopy can be considered a nondestructive technique; however, sometimes the laser power can destroy the sample or change its structure during the measurement. The use of microscopy opens the possibility to search different areas of the sample. In a conventional microscope, it is possible to investigate a very small part of the

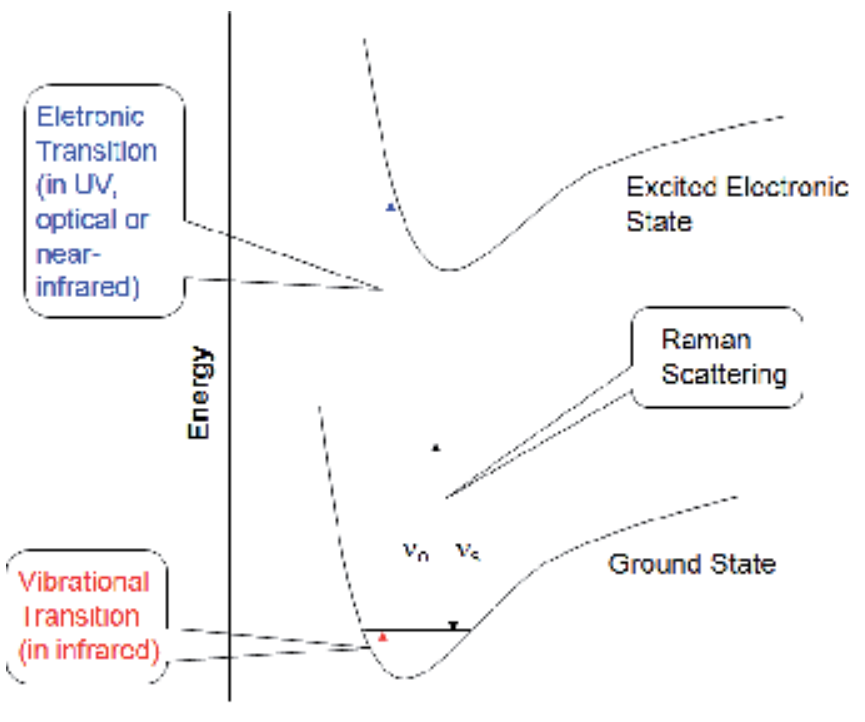

Internuclear separation

Figure 4.

Schematic representation of two electronic states (ground and excited) and their respective vibrational levels (the electronic and vibrational levels are not in the same scale). The arrows indicated the types of transitions among the different levels. For Raman scattering, if the laser line (wavenumber is represented by $\nu_{0}$ ) has energy similar to one electronic transition of the molecule, the signal can be intensified by resonance process, known as resonance Raman effect. In the figure, $\nu_{0}$ and $\nu_{s}$ (the scattered frequency is composed of $\nu_{\text {ev,gm }}$ and $\nu_{e v, g n}$, the stoke and antistoke components, respectively) are the laser line and the scattered frequencies, respectively (for illustration purposes, just the stokes, $\nu_{s}<\nu_{0}$, component is shown in the diagram). 


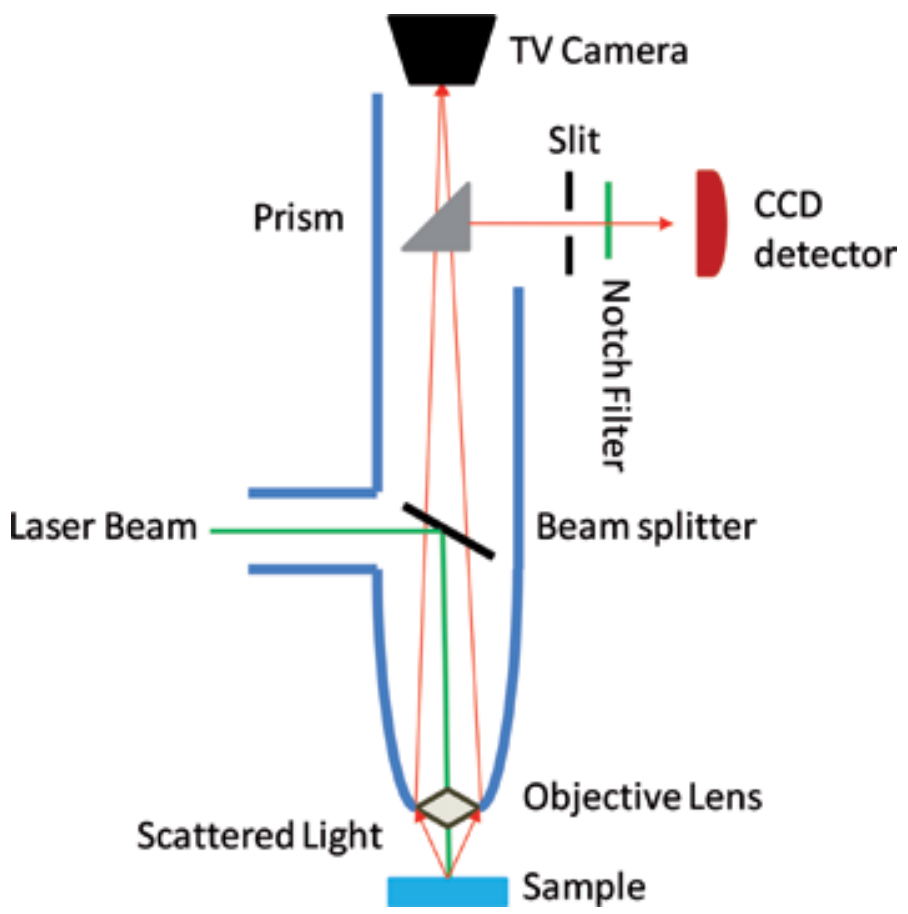

Figure 5.

Conventional Raman microscope.

sample ( $1 \mu \mathrm{m}$ approximately or smaller). The high lateral resolution and depth of field (the order of a few micrometers) are very useful for the study of multilayered polymeric thin films or other complex materials [42-44].

\section{Results and discussion}

\subsection{Resonance Raman of modified single- and double-walled carbon nanotubes}

The resonance Raman spectroscopy technique is sensitive to the electronic, structural, and vibrational properties of CNTs. Our group is using resonance Raman spectroscopy to characterize the interactions between nanotubes and different kinds of molecules [25-39], such as the conducting polyaniline (PANI), molecular magnets such as $\left(\mathrm{NBu}_{4}\right)_{2}[\mathrm{Cu}($ opba $)],[\mathrm{MnCu}(\text { opba })]_{\mathrm{n}}$ chains, where opba $=$ ortho-phenylenebis (oxamate), dyes such as phenosafranine (PS) and Nile Blue (NB), and CNTs doped with bromine or iodine.

For all samples investigated, the resonance Raman spectra are dominated by SWCNT or DWCNT bands at different laser excitation energies $\left(E_{\text {laser }}\right)$ [37-39]. The Raman spectra obtained with laser lines from 790 to $514.5 \mathrm{~nm}$ ( $E_{\text {laser }}$ from 1.57 to $2.41 \mathrm{eV}$ ) are dominated by the characteristic bands from the SWCNTs or DWCNTs. This behavior is associated with the strong resonance Raman with the van Hove singularities of the single-walled nanotubes $[6,7]$. The spectra can be divided into four groups of bands: (1) sharp bands from 120 to $350 \mathrm{~cm}^{-1}$ are assigned to the radial breathing modes (RBMs); (2) strong band in the frequency range from 1500 to $1650 \mathrm{~cm}^{-1}$ is attributed to stretching modes of carbon atoms (tangential $G$ band); (3) the mode at ca. $1300-1350 \mathrm{~cm}^{-1}$ is forbidden for symmetry reasons and is related to the disorder-induced $D$-band feature; and (4) finally, the second-order 
G' band (or 2D band), which is the highly dispersive harmonic of the $D$-band frequency, and it is observed here from ca. 2600 to $2700 \mathrm{~cm}^{-1}$.

As a consequence of the intense CNT bands, the analysis remains in the comparison of standard CNTs before and after any chemical change. For instance, Figure 6 exhibits the Raman spectra, in the RBM region, obtained for the composite between the SWNTs and [MnCu(opba) $]_{\mathrm{n}}$ chains. At higher $E_{\text {laser }}(2.33 \mathrm{eV})$ or $532.0 \mathrm{~nm}$, it was observed many changes in the RBM bands, shifts from 254 to $257 \mathrm{~cm}^{-1}$ $\left(\Delta=+3 \mathrm{~cm}^{-1}\right)$, from 266 to $270 \mathrm{~cm}^{-1}\left(\Delta=+4 \mathrm{~cm}^{-1}\right)$, and from 276 to $281 \mathrm{~cm}^{-1}$ $\left(\Delta=+5 \mathrm{~cm}^{-1}\right)$ were seen for tubes assigned to $E_{11}^{M}$ family with minor diameter than observed for the other laser lines. In Figure 6, it is also observed that RBM bands assigned to $E_{33}^{s}$ family also have changed their intensities and frequencies from 155 to $160 \mathrm{~cm}^{-1}\left(\Delta=+5 \mathrm{~cm}^{-1}\right)$ and from 143 to $149 \mathrm{~cm}^{-1}\left(\Delta=+6 \mathrm{~cm}^{-1}\right)$ in the presence of the heterobimetallic complex.

At $E_{\text {laser }}=2.33 \mathrm{eV}$ also, semiconducting tubes interact with the heterobimetallic polymer, contrarily to that observed for lower $E_{\text {laser }}$ energies. Hence, the RBM data suggest that the heterobimetallic polymer interacts mainly with metallic tubes independently of the diameter of the tube and excitation energy, however, for semiconducting tubes, solely for tubes with diameter higher than ca. $1.47 \mathrm{~nm}$ (Figure 5). In certain circumstances, it is possible to use UV laser line, and some bands from the metallic complex emerge in the spectra. For instance, the resonance Raman spectra, at higher $E_{\text {laser }}(3.82 \mathrm{eV})$, of SWCNTs- $\left(\mathrm{NBu}_{4}\right)_{2}[\mathrm{Cu}$ (opba)] samples show bands at 1430 and $1474 \mathrm{~cm}^{-1}$. These bands can be assigned to the vibrational modes related to the benzene-like ring in the molecular structure of the $\left[\mathrm{Cu}(\text { opba) }]^{2-}\right.$ anion [34].

The CNT families are assigned by using the Kataura plot (Figure 7a). The Kataura plot is the result of calculated energy separations between the van Hove singularities $\left[E_{\mathrm{ii}}\left(d_{\mathrm{t}}\right)\right]$ for SWCNTs obtained from tight-binding calculations [15, $45]$. For instance, according to the Kataura plot, at $514.5 \mathrm{~nm}\left(E_{\text {laser }}=2.41 \mathrm{eV}\right)$ or $532.0 \mathrm{~nm}\left(E_{\text {laser }}=2.33 \mathrm{eV}\right)$, the DWCNTs in resonance have inner tube metallic and outer tube semiconducting. The $\mathrm{Br}_{2}$ doping was reported for SWCNTs $[47,48]$ and for DWCNTs $[49,50]$. A very large Raman bands upshifted at $514.5 \mathrm{~nm}(2.41 \mathrm{eV})$

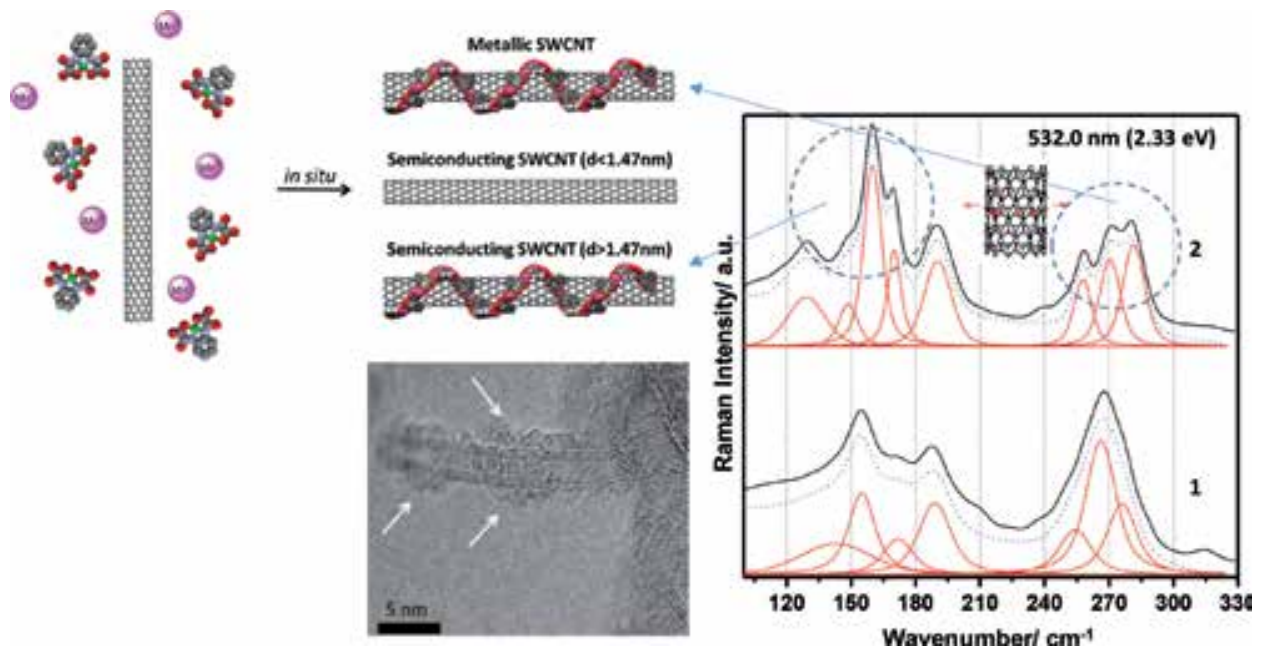

Figure 6.

Schematic representation of the selective interaction scheme between $C N T s$ and $[M n C u(o p b a)]_{n}$ chains formed from the reaction of $\mathrm{Cu}(\mathrm{opba})^{2-}$ and $\mathrm{Mn}^{2+}$ ions. The wrapping is selective for metallic tubes and for semiconducting with diameter higher than $1.47 \mathrm{~nm}$ (the HR-TEM, high resolution transmission microscopy, image of the nanocomposite is also given in the figure). Resonance Raman spectra (RBM band region) excited by laser line at $532.0 \mathrm{~nm}$ (2.33 eV) for powder samples 1 (DWCNTs) and 2 ([MnCu(opba) $]_{n}-S W C N T$ materials). 


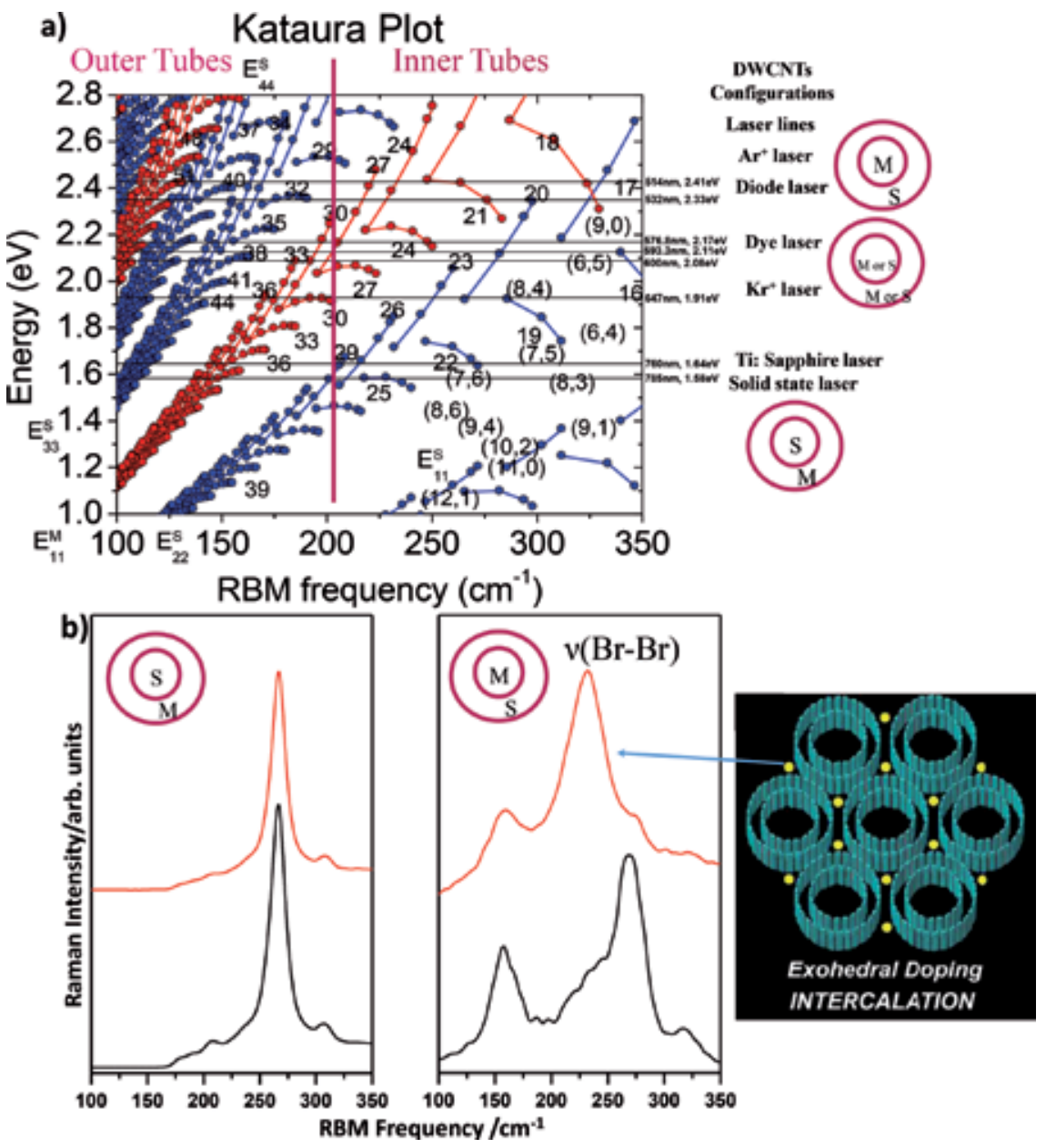

Figure 7.

(a) The Kataura plot at RBM region is based on the extended tight binding model for SWCNTs [15, 45, 46]. The horizontal lines show the laser lines used in the experiments. The numbers indicate the $2 n+m$ families. Red circles correspond to metallic white blue to semiconducting tubes. The diameters of the tubes can be calculated by using the expression $\nu_{R B M}=233 / d t+14$ [46]. (b) Resonance Raman spectra at $532.0 \mathrm{~nm}(2.33 \mathrm{eV})$ of DWCNTs before and after doping (black and red lines, respectively) with 31 wt\% of $\mathrm{Br}_{2}$. For illustrative purpose, the schematic representation of the exohedral doping is also given in the figure.

laser line in both the RBM and $\mathrm{G}^{+}$band for brominated SWCNTs are observed. Indeed, the doping induces a Fermi level depression of 1.2-1.4 eV in the DWCNTs after bromination, mainly in the outer tubes. The $\mathrm{Br}-\mathrm{Br}$ molecular vibration is reduced to ca. $233 \mathrm{~cm}^{-1}$ in comparison with molecular bromine $\left(323 \mathrm{~cm}^{-1}\right)$ [37-39], as a consequence to the charge transfer with the carbon tubes.

The use of a large variety of laser lines [37-39] permits to monitor differences in the charge transfer behavior to the inner and outer tubes from the adsorbed bromine or iodine. Figure $7 \mathbf{b}$ shows that the $\mathrm{Br}_{2}$ molecules are interacting with the outer tube surface of the DWCNTs and that the adsorbed bromine molecules act as an electron acceptor. In addition, it is observed that metallic tubes are extremely sensitive to doping process. In fact, the presence of $\mathrm{Br}_{2}$ molecules changes the Raman spectra of the metallic tubes even when they are in the inner configuration surrounded by semiconducting outer tubes of DWCNTs.

The differences between metallic and semiconducting tubes can also be analyzed in the other regions of the Raman spectra of CNTs. For instance, the presence of the lower frequency component of the G-band spectra is associated with the 

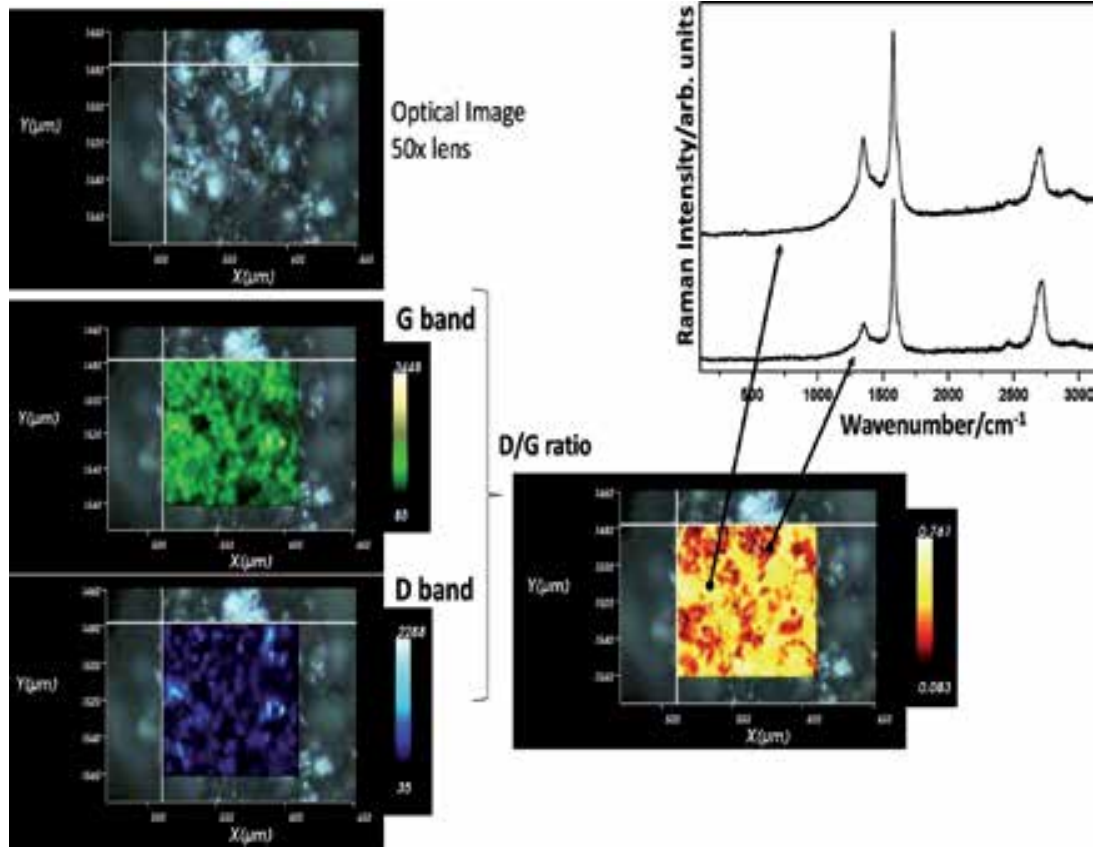

Figure 8.

Resonance Raman imaging of commercial samples of graphene. The images on left are from the top: optical image obtained with a $50 \times$ lens, the Raman imaging using the $G$ band as intensity marker (green image), and the Raman imaging using the $D$ band as intensity marker (blue image). In addition, the Raman imaging obtained from $D / G$ ratio is also given (red-yellow image) and the corresponding resonance Raman spectra from red and yellow regions.

metallic tubes. This is the result of the strong electron-phonon coupling observed in metallic nanotubes, and it gives rise to Kohn anomalies in the phonon dispersions $[51,52]$. Hence, the influence of the metallic tubes can easily be distinguished from semiconducting $G^{-}$feature, which shows a Breit-Wigner-Fano (BWF) line shape $[53,54]$. The changes in relative intensities, dispersion, and linewidth of the G' (2D) band can also be used as a probe to extract information about interactions between the tubes and the graphene layers.

\subsection{Raman imaging of commercial sample of graphene}

More recently, our group is dedicated to prepare modified graphene samples with a myriad of molecules. However, due to the limitations of chemical procedures for the preparation of chemically modified graphene, the resulting samples are very inhomogeneous. Graphene consists in a two-dimensional single-layer sheet of $\mathrm{sp}^{2}$ hybridized carbon atoms having excellent electron transport properties and very high carrier mobility and thermal conductivity. However, the interesting properties exhibited by graphene are only observed for graphene films that contain only one or a few graphene layers [3, 4]. It is optically difficult to observe low number of layers, but single-, double-, and multilayer graphenes can be differentiated by their Raman fingerprints. The graphene Raman spectrum is dominated by $\mathrm{G}$ band (ca. $1582 \mathrm{~cm}^{-1}$ ) and 2D band (ca. $2685 \mathrm{~cm}^{-1}$ ). Ferrari and coworkers [55] used Raman to characterize samples consisting of varying number of graphene layers and found that the 2D band width and shape modify with increasing the number of layers. Therefore, the spectral shape of the $2 \mathrm{D}$ band is representative of the number of graphene layers and can be used to determine that number. By using 
Raman imaging has been possible to analyze a large area of the graphene samples in order to search irregularities. For example, Figure 8 shows the Raman imaging obtained from a commercial sample of graphene (Sigma-Aldrich). The D- and G-band intensities were used as probe to see the differences in a large area of the sample.

The $\mathrm{D}$ and $\mathrm{G}$ images can be combined to form an image from the $\mathrm{D} / \mathrm{G}$ ratio of intensities (red-yellow image). The Raman spectra of two spots of the sample exemplify the point; yellow regions have more intense $\mathrm{D}$ bands than red regions.

\section{Conclusion and future remarks}

The screening of the electronic and vibrational structure of carbon allotropes through Raman spectroscopy has been decisive in determination of their structure and in the study of the interactions between the carbon allotropes with a myriad of other molecules. In fact, by selecting the appropriate laser line, it is possible to study in particular metallic or semiconducting tubes or monolayer graphenes. The new Raman instruments can give better Raman imaging of the samples and open the possibility to study inhomogeneity, chemical modifications, and many other aspects of the carbon samples in a large area.

\section{Author details}

Gustavo Morari do Nascimento

Federal University of ABC, Santo André, Brazil

*Address all correspondence to: Gustavo.morari@ufabc.edu.br; morari@yahoo.com

IntechOpen

(C) 2020 The Author(s). Licensee IntechOpen. This chapter is distributed under the terms of the Creative Commons Attribution License (http://creativecommons.org/licenses/ by/3.0), which permits unrestricted use, distribution, and reproduction in any medium, provided the original work is properly cited. (cc) BY 


\section{References}

[1] Ozin GA, Arsenault AC.

Nanochemistry: A Chemical Approach to Nanomaterials. Cambridge: RSC

Publishing; 2005

[2] Ijima S. Helical microtubules of graphitic carbon. Nature. 1991;354:56

[3] Novoselov KS, Geim AK, Morozov SV, Jiang D, Zhang Y, Dubonos SV, et al. Electric field effect in atomically thin carbon films. Science. 2004;306:666

[4] Geim AK, Novoselov KS. The rise of graphene. Nature Materials. 2007;6:183

[5] Kroto HW, Heath JR, O'Brien SC, Curl RF, Smalley RE. C60:

Buckminsterfullerene. Nature. 1985;318:162

[6] Saito R, Dresselhaus G, Dresselhaus MS. Physical Properties of Carbon Nanotubes. London: Imperial College Press; 1998

[7] Dresselhaus MS, Dai H. Carbon nanotubes: Continued innovations and challenges. MRS Bulletin. 2004;29:237

[8] Jorio A, Saito R, Hertel T, Weisman RB, Dresselhaus G, Dresselhaus MS. Carbon nanotube photophysics. MRS Bulletin. 2004;29:276

[9] Saito R, Fujita M, Dresselhaus G, Dresselhaus MS. Electronic structure of graphene tubules based on C60. Physical Review B. 1992;46:1804

[10] Mintmire JW, Robertson DH, White CT. Properties of fullerene nanotubules. Journal of Physics and Chemistry of Solids. 1993;54:1935

[11] Ijima S, Ichihashi T. Single-shell carbon nanotubes of 1-nm diameter. Nature. 1993;363:603
[12] Bethune DS, Kiang CH, de Vries MS, Gorman G, Savoy R, Vazquez J, et al. Cobalt-catalysed growth of carbon nanotubes with single-atomic-layer walls. Nature. 1993;363:605

[13] Dresselhaus MS, Dresselhaus G, Avouris P. Carbon Nanotubes: Synthesis, Structure, Properties and Applications. Berlin: Springer-Verlag; 2001

[14] Dresselhaus MS, Dresselhaus G, Jorio A, Souza Filho AG, Pimenta MA, Saito R. Single nanotube Raman spectroscopy. Accounts of Chemical Research. 2002;35:1070

[15] Jorio A, Saito R, Hafner JH, Lieber CM, Hunter M, McClure T, et al. Structural $(\mathrm{n}, \mathrm{m})$ determination of isolated single-wall carbon nanotubes by resonant Raman scattering. Physical Review Letters. 2001;86:1118

[16] Qujang M, Huang J-L, Lieber CM. Fundamental electronic properties and applications of single-walled carbon nanotubes. Accounts of Chemical Research. 2002;35:1018

[17] Chung D. Review: graphite. Journal of Materials Science. 2002;37:1475

[18] Rao CNR, Sood AK, Subrahmanyam KS, Govindaraj A. Graphene: the new two-dimensional nanomaterial. Angewandte Chemie, International Edition. 2009;48:7752

[19] Pati SK, Enoki T, Rao CNR. Graphene and its Fascinating Attributes. Singapore: World Scientific; 2011

[20] Wallace PR. The band theory of graphite. Physics Review. 1947;71:622

[21] Dreyer DR, Ruoff RS, Bielawski CW. From conception to realization: An historial account of graphene and some perspectives for its future. Angewandte 
Chemie, International Edition. 2010;49:9336

[22] Novoselov KS, Jiang D, Schedin F, Booth TJ, Khotkevich VV, Morozov SV, et al. Proceedings of the National Academy of Sciences of the United States of America. 2005;102:10451

[23] Pendolino F, Armata N. Graphene Oxide in Environmental Remediation Process. 1st ed. Itália: Springer; 2017

[24] Hummers S, Offeman RE. Preparation of graphitic oxide. Journal of the American Chemical Society. 1958;80(6):1339

[25] Bonancea CE, do Nascimento GM, de Souza ML, Temperini MLA, Corio P. Substrate development for surface-enhanced Raman study of photocatalytic degradation processes: Congo red over silver modified titanium dioxide films. Applied Catalysis B: Environmental. 2006;69:34-42

[26] Silva IF, Teixeira IF, Barros WP, Pinheiro CB, Ardisson JD, do Nascimento GM, et al. An FeIII dinuclear metallacycle complex as a sizeselective adsorbent for nitrogenous compounds and a potentially effective ammonia storage material. Journal of Materials Chemistry A. 2019;7:15225-15232

[27] do Nascimento GM, do Pim WD, Reis DO, Simoes TRG, Pradie NA, Stumpf HO. Characterization of compounds derived from copperoxamate and imidazolium by $\mathrm{X}$-ray absorption and vibrational spectroscopies. Spectrochimica Acta Part A. 2015;142:303-310

[28] do Nascimento GM, Corio P, Novickis RW, Temperini MLA, Dresselhaus MS. Synthesis and characterization of single-wall-carbonnanotube-doped emeraldine salt and base polyaniline nanocomposites. Journal of Polymer Science, Part A: Polymer Chemistry. 2005;43:815
[29] do Nascimento GM, Silva TB, Corio P, Dresselhaus MS. Chargetransfer behavior of polyaniline single wall carbon nanotubes nanocomposites monitored by resonance Raman spectroscopy. Journal of Raman Specroscopy. 2010;41:1587

[30] do Nascimento GM, Constantino VRL, Temperini MLA. Spectroscopic characterization of a new type of conducting polymer-clay nanocomposite. Macromolecules. 2002;35:7535

[31] do Nascimento GM, Constantino VRL, Temperini MLA. Spectroscopic characterization of doped poly(benzidine) and its nanocomposite with cationic clay. The Journal of Physical Chemistry B. 2004;108:5564

[32] do Nascimento GM, Constantino VRL, Landers R, Temperini MLA. Aniline polymerization into montmorillonite clay: A spectroscopic investigation of the intercalated conducting polymer. Macromolecules. 2004;37:9373

[33] Do Nascimento GM, do Pim WD, Endo M, Choi GB, Kim YA, Pradie NA, et al. Single-wall carbon nanotube modified with copper-oxamate flat complex probed by synchrotron x-ray photoelectron and $x$-ray absorption spectroscopies. Journal of Molecular Structure. 2019;1176:711-719

[34] Silva IF, do Pim WD, Teixeira IF, Barros WP, Teixeira APC, Do Nascimento GM, et al. Selective wrapping of few-walled carbon nanotubes by a serpent-like heterobimetallic coordination polymer. Journal of Physical Chemistry C. 2016;120:1245

[35] do Nascimento GM, Barros WP, Kim YA, Muramatsu H, Hayashi T, Endo M, et al. Single-wall carbon nanotube interactions with copper-oxamato building block of 
moleculebased magnets probed by resonance Raman spectroscopy. Journal of Raman Specroscopy. 2012;43:1951

[36] do Nascimento GM, de Oliveira RC, Pradie NA, Lins PRG, Worfel PR, Martinez GR, et al. Single-wall carbon nanotubes modified with organic dyes: Synthesis, characterization and potential cytotoxic effects. Journal of Photochemistry and Photobiology A: Chemistry. 2010;211(2-3):99-107

[37] do Nascimento GM, Hou T, Kim YA, Muramatsu H, Hayashi T, Endo M, et al. Behavior of the high frequency Raman modes of double-wall carbon nanotubes after doping with bromine or iodine vapors. Carbon. 2011;49:3585-3596

[38] do Nascimento GM, Hou T, Kim YA, Muramatsu H, Hayashi T, Endo M, et al. Double-wall carbon nanotubes doped with different Br2 doping levels: A resonance Raman study. Nano Letters. 2008;8(12):4168-4172

[39] do Nascimento GM, Hou T, Kim YA, Muramatsu H, Hayashi T, Endo M, et al. Comparison of the resonance raman behavior of double-walled carbon nanotubes doped with bromine or iodine vapors. Journal of Physical Chemistry C. 2009;113(10):3934-3938

[40] Nakamoto N, Ferraro JR. Introduction to Raman Spectroscopy. London: Academic Press, Inc.; 1994

[41] Rostron P. D. Gerber Raman spectroscopy, a review. International Journal of Engineering and Technical Research. 2016;6:50-64

[42] Batchelder DN. In: Brässler H, editor. Optical Techniques to Characterize Polymer Systems. Amsterdam: Elsevier; 1987

[43] Batchelder DN, Bloor D. In Advances in Infrared and Raman Spectroscopy. Wiley-Heyden: London; 1984
[44] Clark JH, Dines TJ. Resonance raman spectroscopy, and its application to inorganic chemistry. New Analytical Methods (27). Angewandte Chemie (International Ed. in English). 1986;25:131

[45] Kataura H, Kumazawa Y, Maniwa Y, Umezu I, Suzuki S, Ohtsuka Y, et al. Optical properties of single-wall carbon nanotubes. Synthetic Metals. 1999;103(1-3):2555-2558

[46] Pfeiffer R, Kramberger C, Simon F, Kusmany H, Popov VN, Kataura H. Interaction between concentric tubes in DWCNTs. European Physical Journal B. 2004;42:345-350

[47] Lee RS, Kim HJ, Fischer JE, Thess A, Smalley RE. Conductivity enhancement in single-walled carbon nanotube bundles doped with $\mathrm{K}$ and $\mathrm{Br}$. Nature. 1997;388(6639):255-257

[48] Rao AM, Eklund PC, Bandow S, Thess A, Smalley RE. Evidence for charge transfer in doped carbon nanotube bundles from Raman scattering. Nature. 1997;388(6639):257-259

[49] Chen G, Bandow S, Margine ER, Nisoli C, Olmogorov AN, Crespi VH, et al. Chemically doped doublewalled carbon nanotubes: Cylindrical molecular capacitors. Physical Review Letters. 2003;90(25):257403-257404

[50] Souza Filho AG, Endo M, Muramatsu H, Hayashi T, Kim YA, Barros EB. et al. Resonance Raman scattering studies in Br2-adsorbed double-wall carbon nanotubes. Physical Review B.2006;73(23):235413235411-12

[51] Piscanec S, Lazzeri M, Robertson J, Ferrari AC, Mauri F. Optical phonons in carbon nanotubes: Kohn anomalies, Peierls distortions, and dynamic effects. Physical Review B. 2007;75(3):035427 
[52] Basko DM, Piscanec S, Ferrari AC.

Electron-electron interactions and doping dependence of the two-phonon Raman intensity in graphene. Physical ReviewB.2009;80(16):165413-165411-10

[53] Souza Filho AG, Jorio A, Samsonidze GG, Dresselhaus G, Pimenta MA, Dresselhaus MS et al. Competing spring constant versus double resonance effects on the properties of dispersive modes in isolated single-wall carbon nanotubes. Physical Review B.2003;67(3):035427- 035421-7

[54] Jorio A, Souza Filho AG, Dresselhaus G, Dresselhaus MS, et al. G-band resonant Raman study of 62 isolated single-wall carbon nanotubes. Physical Review B. 2002;65(15):155412-155419

[55] Ferrari AC, Meyer JC, Scardaci V, Casiraghi C, Lazzeri M, Mauri F, et al. Raman spectrum of graphene and graphene layers. Physical Review Letters. 2006;97:187401 


\title{
Chapter 7
}

\section{Quantitative Analysis by IR: Determination of Chitin/ Chitosan DD}

\author{
Boukhlifi Fatima
}

\begin{abstract}
FTIR spectroscopy has been widely used to quantitatively study the parameters of the chitin deacetylation. A new research on a Canadian chitin has shown that a degree of deacetylation (DD) of $90 \%$ has been reached with a base concentration of $12.5 \mathrm{M}$, a reaction time of $120 \mathrm{~min}$, and a temperature of $110^{\circ} \mathrm{C}$. In parallel, our study on Moroccan chitin allowed to reach $75 \%$. A degree of deacetylation of $75 \%$ was obtained at $\mathrm{T}=120^{\circ} \mathrm{C}$ and at $\mathrm{C}_{\mathrm{NaOH}}=12 \mathrm{~N}$ in a single step for 6 hours. Another study followed by IR prepared the chitosan under pressure or under irradiation. Firstly, the compression method was used for preparing $100 \%$ deacetylated chitosan with less environmental pollution. The $100 \%$ fully deacetylated chitosan is produced in lowconcentration alkali and high-pressure conditions under 0.11-0.12 MPa for $120 \mathrm{~min}$. Secondly, microwave deacetylation showed that a degree of deacetylation of $95.19 \%$ was achieved after irradiating chitin at 60 meshes with $50 \% \mathrm{NaOH}$ solution in a microwave for $10 \mathrm{~min}$ at $1400-\mathrm{W}$ power. To find these results, the authors used different formulas to calculate DD by FTIRM, but the most used and reliable formula is that which calculates DD of chitosan by the report of absorbance of amide at $1655 \mathrm{~cm}^{-1}$ that measures the acetyl group and absorbance at $3430 \mathrm{~cm}^{-1}$ relating to the hydroxyl group.
\end{abstract}

Keywords: chitin, chitosan, DD, FTIR, optimization, synthesis

\section{Introduction}

Very commonly used in quantitative analysis, infrared spectrometry FTIR has been exploited in many fields. It has been used in mineralogy in addition to chemical analyzes to determine the structure of a rock and to know the bonds between the atoms, in pharmaceutical for the quantitative measurement of the constituents and the thickness of the coating of the tablets, in dairy industries to determine the moisture of milk powders and butters, in the textile industry for fiber sizing and maturity of cottons, in the chemical industry, in agribusiness, and in several other industries. When a specific absorption of a chemical function of a complex molecule is sufficiently isolated in its spectrum, it is always possible to carry out quantitative measurements. There are several major fields of application for infrared spectrophotometry. First, we can quote functional analysis is probably the main application of infrared spectrometry, at least in industry. Then, the structural analysis, infrared spectrometry allows to obtain even more fine information, concerning the 
"construction of the molecular edifice." In organic compounds, it allows for example to differentiate the isomers of position (ortho-, meta-, and para-) aromatic hydrocarbons, as well as the cis- and transisomers of olefins. For mineral compounds, the infrared spectrum depends on the symmetry of molecules; it often allows finding the system in which a chemical compound is crystallized. It is also possible, but only in the case of small molecules, to calculate geometric parameters such as moments of inertia. The analysis is done by comparing with reference spectra of which there are several files. Also, FTIR spectroscopy is the method most used for calculating the degree of DD of chitosan. This polymer was discovered in 1859 by C. Rouget by treating chitin with concentrated $\mathrm{KOH}$ at elevated temperature. But it was not until 1894 that Hoppe-Seyler gave the "modified chitin" the name chitosan [1]. Chitosan has some advantageous properties, such as biocompatibility, biodegradable polymer of high molecular weight, nontoxic, and antimicrobial activity, that encourage its applications in many fields including agriculture $[2,3]$, paper industry, food and textile industries, pharmaceutics $[4,5]$, biochemistry, biotechnology, cosmetics, biomedical applications [6-8], environment, and water treatment [9-13]. The properties of chitin and chitosan depend considerably on the degree of deacetylation (DD), a parameter defined as the mole fraction of deacetylated units in the polymer chain $[14,15]$. Therefore, the determination of DD has been one of the interesting parameters to study chitosan preparations. The process of deacetylation involves the removal of acetyl groups from the molecular chain of chitin, leaving behind a complete amino group $\left(-\mathrm{NH}_{2}\right)$, and chitosan properties are very linked on this high degree of chemical reactive amino groups. Since the degree of deacetylation (DD) depends mainly on the method of purification and reaction conditions, it is therefore essential to characterize chitosan by determining its DD prior to its use. The main parameters involved in the process are temperature, time of reactions, and the concentration of reagents. A simple and nonexpensive chemical treatment of mineral/protein removal from chitin is usually used with $\mathrm{HCl} / \mathrm{NaOH}$ reagents, respectively, and chitosan is chemically or enzymatically produced. They vary only on the acetyl group container, which is designated by the degree of acetylating (DA) designating the percentage of acetylated units relative to the number of total units. The term chitosan applies to any copolymer whose DD is greater than $50 \%$. Each chitosan is therefore characterized by the fraction of residual $\mathrm{N}$-acetamide groups (DA) or by the relative amount of amino groups of the chitosan molecule (DD =1-DA) [16]. It is important to distinguish between the degree of acetylation (DA) and the degree of deacetylation (DD). One being the opposite of the other, that is to say that chitosan having an $85 \% \mathrm{DD}$, it has $15 \%$ of acetyl groups and $85 \%$ of amine groups on its chains. The degree of deacetylation (DD) of chitosan is a dominant structural parameter that significantly influences the physicochemical properties of chitosan such as solubility, overall charge, reactivity, and mechanical properties such as elongation, breaking, and tensile strength. This parameter also influences biological properties [17] such as biocompatibility and biodegradability. For determination of the degree of deacetylation (DD), several analytical methods have been employed. Infrared spectroscopy $[18,19]$ and UV spectrophotometry [20] as analytical tools offer advantage over other traditional techniques which are expensive and destructive to the sample. FTIR spectroscopy is a quick technique for a quantitative evaluation of the DD through the determination of absorption ratios. FTIR analysis is attractive due to its nondestructive character, fastness, sensitivity, and suitability for both soluble and nonsoluble samples. Among the solution methods, first-derivative UV spectrophotometry draws attention owing to its simplicity and effectiveness in providing accurate results for highly deacetylated chitin. It was conceived by Muzzarelli and 
Rocchetti [20] and relies on simple reagents and instrumentation. In addition, the results obtained from this method are reasonably independent of protein contamination. Alonso et al. [21] established the possibility to determine the acetylation degree with the use of empirical correlations based on the weight losses associated with the main decomposition peaks. A similar approach has been adopted to investigate if there was any relationship between the weight loss of the sample and its DD. According to Yu et al. [22], the conductometric assay is an adequate and accurate method for determining the degree of deacetylation of chitosan, except for some samples that have a high degree of crystallization. The conductometric method can be carried out in basic and acid medium. Other methods have also been used such as SEM and NMR for magnetic properties of certain atomic nuclei and the determination of DD.

The objective of this chapter is to present a bibliographic synthesis on the use of spectroscopy FTIR in order to study and optimize the reaction of deacetylation by calculating the chitosan DD. At the end of the chapter, a simple comparison between IR and other methods of DD determination is presented to find the most reliable formula of DD calculation.

\section{How to extract chitin}

Chitin is the structural polymer of exoskeletons of all arthropods (crustaceans and insects) and endoskeletons of cephalopods (cuttlefish, squid, etc.). The cuticles of various crustaceans, mainly crabs and shrimp, are the main sources of raw material for the production of chitin (Table 1). Chitin is found as part of a complex network of proteins on which calcium carbonate is deposited to form the rigid shell in crustaceans or more specifically in shellfish. The interaction between chitin and proteins is very intimate and there is also a small fraction of proteins involved in a polysaccharide-protein complex [24]. Thus, the preparation of chitin from shellfish requires the elimination of the two main constituents, namely proteins by deproteinization and calcium carbonate by demineralization, as well as small amounts of pigments and lipids generally removed during the two steps. An additional fading step is applied to remove residual pigments. Many methods have been proposed and used over the years to prepare pure chitin; however, no standard method has been

\begin{tabular}{llll}
\hline Lower plants & Annelid & Mushrooms & Molluscs \\
\hline Algae Lichen yeasts & $\begin{array}{l}\text { Ascomycetes (Class) } \\
\text { Penicillium } \\
\text { Blastocladiales (Family) } \\
\text { Chytridiaceae (Family) }\end{array}$ & Earthworm Leech & Cuttlefish Octopus \\
& & \\
\hline Arthropods & Arachnids & Insects & \\
\hline Crustaceans & Octopus & Spiders & \\
\hline Lobsters & Scorpions & Ants \\
Crabs & & Cockroaches & \\
Shrimps & & Coleoptera (Order) & \\
Scampi & & & \\
Krill & & & \\
\hline
\end{tabular}

Table 1.

Sources of chitin [25]. 
adopted. Deproteinization and demineralization can be carried out using chemical or enzymatic treatments. In the case of shrimp, the shell wall is thinner, which facilitates the isolation of chitin compared to other types of shells. The selected shells are then cleaned, dried, and ground into small shell pieces. Shrimp carapaces have the following average mass composition:

- $75 \%$ water

- $12 \%$ protein

- $9 \%$ mineral salts

- $4 \%$ chitin

- traces of lipids and organic pigments

We then develop the two essential steps for the preparation of chitin from the carapaces, namely deproteinization and demineralization.

\subsection{Chemical deproteinization}

The deproteinization of chitin consists in eliminating proteins; it is difficult because there is a breakdown of the chemical bonds between chitin and proteins. This is done using basic solutions in a heterogeneous way. Complete protein isolation is particularly important for biomedical applications. A wide range of chemicals have been tested as deproteinization reagents, including $\mathrm{NaOH}, \mathrm{Na}_{2} \mathrm{CO}_{3}, \mathrm{NaHCO}_{3}$, $\mathrm{KOH}, \mathrm{K}_{2} \mathrm{CO}_{3}, \mathrm{Ca}(\mathrm{OH})_{2}, \mathrm{Na}_{2} \mathrm{SO}_{3}, \mathrm{NaHSO}_{3}, \mathrm{CaHSO}_{3}, \mathrm{Na}_{3} \mathrm{PO}_{4}$, and $\mathrm{Na}_{2} \mathrm{~S}$. The reaction conditions vary considerably in each study. $\mathrm{NaOH}$ is the preferred reagent and is applied at a concentration ranging from 0.125 to $12 \mathrm{M}$, at different temperatures (up to $20^{\circ} \mathrm{C}$ ) and a duration of treatment (from a few minutes to a few days). In addition to deproteinization, the use of $\mathrm{NaOH}$ results in partial deacetylation of chitin and hydrolysis of the biopolymer, which decreases its molecular weight.

\subsection{Chemical demineralization}

Demineralization is a necessary step to produce chitosan. It consists of dissolving minerals, mainly calcium carbonate bound to chitin. Demineralization is generally carried out by acid treatment using $\mathrm{HCl}, \mathrm{HNO}_{3}, \mathrm{H}_{2} \mathrm{SO}_{4}, \mathrm{CH}_{3} \mathrm{COOH}$, and $\mathrm{HCOOH}$ $[26,27]$. Of these acids, the preferred reagent is dilute hydrochloric acid. Demineralization is an acid-base reaction between carbonate ions and acids in water with the release of carbon dioxide, as indicated in the following equation:

$$
2 \mathrm{HCl}+\mathrm{CaCO}_{3} \rightarrow \mathrm{CaCl}_{2}+\mathrm{H}_{2} \mathrm{O}+\mathrm{CO}_{2}
$$

All the other minerals present in the crustacean cuticle react in the same way and give soluble salts in the presence of acid. Then, the salts can be easily separated by filtering the solid phase of chitin, followed by washing with distilled water.

Chemically, a kilogram of fresh shells provides about $40 \mathrm{~g}$ of dry chitin. After the chemical treatment, the grinding and sieving processes to obtain a homogeneous chitin cause losses of about $40 \%$. The final yield is $2.5 \%, 25 \mathrm{~g}$ of chitin per kilogram of shell. Table 2 summarizes the operating conditions of chitin extraction according to different sources. 
Quantitative Analysis by IR: Determination of Chitin/Chitosan DD

DOI: http://dx.doi.org/10.5772/intechopen.89708

\begin{tabular}{|c|c|c|c|c|c|c|c|c|}
\hline \multirow[b]{2}{*}{ Source } & \multicolumn{4}{|c|}{ Deproteinization } & \multicolumn{4}{|c|}{ Demineralization } \\
\hline & $\mathrm{C}_{\mathrm{NaOH}}{ }^{*}$ & $\mathrm{~T}^{\circ} \mathrm{C}$ & $\begin{array}{c}\text { Number of } \\
\text { baths }\end{array}$ & $\begin{array}{l}\text { Duration } \\
\text { (h) }\end{array}$ & $\mathrm{C}_{\mathrm{HCL}}$ & $\mathrm{T}^{\circ} \mathrm{C}$ & $\begin{array}{c}\text { DURAT-ION } \\
(\mathbf{h})\end{array}$ & \\
\hline Shrimp & $0.125 \mathrm{M}$ & 100 & 1 & 0.5 & $1.25 \mathrm{M}$ & Room & 1 & [28] \\
\hline & $0.75 \mathrm{M}$ & 100 & 1 & 0.5 & & & & \\
\hline Shrimp & $1.25 \mathrm{M}$ & 100 & 1 & 0.5 & $1.57 \mathrm{M}$ & $20-22$ & $1-3$ & [29] \\
\hline Shrimp & $1 \%$ & 65 & 1 & 1 & $0.5 \mathrm{M}$ & Room & - & [30] \\
\hline Shrimp & $3 \%$ & 100 & 1 & 1 & $1 \mathrm{M}$ & Room & 0.5 & [31] \\
\hline Shrimp & $4 \%$ & 100 & 1 & 1 & $5 \%$ & Room & - & [32] \\
\hline Shrimp & $\begin{array}{c}7.5- \\
12.5 \mathrm{M}\end{array}$ & & 1 & 30-180 mn & $0.3-3.5 \mathrm{M}$ & Room & 24 & [23] \\
\hline Shrimp & $1 \mathrm{M}$ & Room & 1 & $24 \mathrm{~h}$ & $1 \mathrm{M}$ & Room & 24 & [3] \\
\hline Crab & $0.5 \mathrm{M}$ & 65 & 1 & 2 & $1.57 \mathrm{M}$ & Room & 5 & [33] \\
\hline Crab & $1 \mathrm{M}$ & 80 & 1 & 3 & $1 \mathrm{M}$ & Room & 12 & [34] \\
\hline Crab & $1 \mathrm{M}$ & 100 & 1 & 36 & $2 \mathrm{M}$ & Room & 48 & [28] \\
\hline Crab & $1 \mathrm{M}$ & 100 & 3 & 72 & $1 \mathrm{M}$ & Room & - & [35] \\
\hline Crab & $1.25 \mathrm{M}$ & $\begin{array}{c}85- \\
90\end{array}$ & 3 & 24 & $1.37 \mathrm{M}$ & Room & 24 & [36] \\
\hline Crab & $1 \mathrm{M}$ & 50 & 1 & 6 & $1 \mathrm{M}$ & 20 & 3 & [37] \\
\hline $\begin{array}{l}\text { Crab/ } \\
\text { lobster }\end{array}$ & $2.5 \mathrm{M}$ & Room & 3 & 72 & $11 \mathrm{M}$ & -20 & 4 & [38] \\
\hline Lobster & $1 \mathrm{M}$ & 100 & 5 & 12 & $2 \mathrm{M}$ & Room & 5 & [39] \\
\hline Lobster & $5 \%$ & $\begin{array}{c}80- \\
85\end{array}$ & 2 & 0.5 & $5 \%$ & 70 & 4 & [40] \\
\hline Lobster & $10 \%$ & 100 & 1 & 2.5 & $\begin{array}{c}10 \% \mathrm{HCl} \\
90 \% \\
\text { formic }\end{array}$ & Room & 18 & [41] \\
\hline Krill & 0.875 & $\begin{array}{c}90- \\
95\end{array}$ & 1 & 2 & $0.6 \mathrm{M}$ & Room & 2 & [42] \\
\hline Krill & $3.5 \%$ & 25 & 1 & 2 & $3.5 \%$ & 20 & 1.5 & [43] \\
\hline Crawfish & & & & & & Room & & [44] \\
\hline
\end{tabular}

Table 2.

Chitin extraction conditions.

\section{How to extract chitosan from chitin}

Chitosan represents a family of polymers obtained at varying degrees after deacetylation of chitin. In fact, the degree of acetylation (DA), which reflects the balance between the two types of residues (Figure 1), differentiates chitin from chitosan. When the DA (expressed as molar percentage) is less than $50 \mathrm{~mol} \%$, the product is called chitosan, and it is characterized by its solubility in acidic solutions [45]. During deacetylation, the amides are protonated and the acetyl groups are removed, but a depolymerization reaction, indicated by the changes in the molecular weight of the chitosan, is also produced.

Chitin can be converted to chitosan by enzymatic preparations [46-49] or by a chemical process $[50,51]$. Chemical methods are widely used for commercial purposes for the preparation of chitosan because of their low cost and their ability to mass produce [51]. 


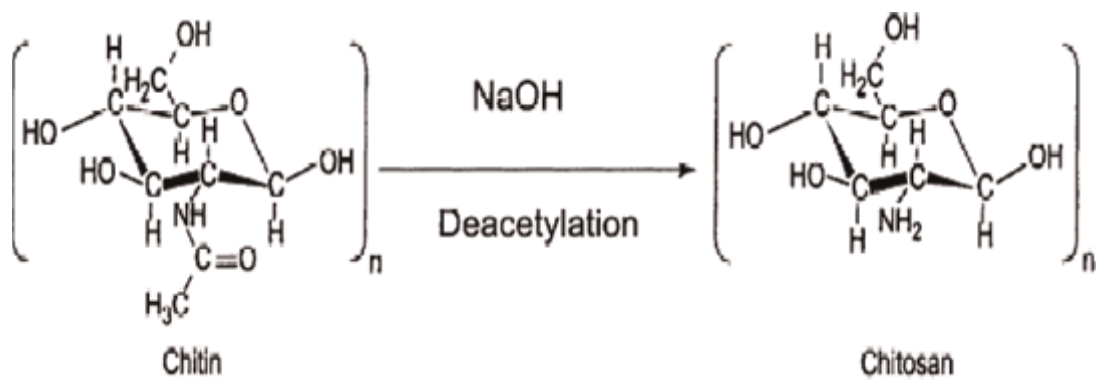

Figure 1.

Equation of chitin conversion to chitosan by deacetylation.

\subsection{Chemical deacetylation}

To deacetylate chitin chemically, acids or bases are used. However, glycosidic bonds are very sensitive to acid treatment [51, 52]; therefore, alkaline deacetylation is often used. The deacetylation reaction is heterogeneous [53] or homogeneous [54]. Generally, in the heterogeneous process, chitin is treated with hot concentrated $\mathrm{NaOH}$ solution for a few hours and chitosan is produced as an $85-99 \%$ deacetylated insoluble residue. According to the homogeneous method, the alkaline chitin is prepared after dispersion of the chitin in concentrated $\mathrm{NaOH}\left(30 \mathrm{~g} \mathrm{NaOH} / 45 \mathrm{~g} \mathrm{H}_{2} \mathrm{O} / 3 \mathrm{~g}\right.$ Chitin) at $25^{\circ} \mathrm{C}$ for $3 \mathrm{~h}$ or more and then dissolved in crushed ice around $0{ }^{\circ} \mathrm{C}$. This method gives a soluble chitosan with an average degree of acetylation of 48-55\% [50]. This process produces a deacetylation with acetyl groups uniformly distributed in the chains, for example chitosan with DA $=10 \%$ after $580 \mathrm{~h}$ at $25^{\circ} \mathrm{C}$ [54].

The details of the process parameters employed by various researchers that include the number of demineralization, deproteinization, and deacetylation step have been reviewed and summarized in Table 3 .

\subsection{Enzymatic deacetylation}

The main disadvantages of chemical deacetylation are energy consumption, waste concentrated alkaline solutions and thus increased environmental pollution. In order to avoid these disadvantages, an alternative enzymatic method exploiting chitin deacetylases has been explored. The use of chitin deacetylase offers the possibility of a nondegradable controlled process, leading to the production of welldefined chitosan [56]. This method is especially used to prepare chitosan oligomers.

Chitin deacetylase catalyzes the hydrolysis of $\mathrm{N}$-acetamido linkages in chitin to produce chitosan. The presence of this enzymatic activity has been reported in several fungi $[57,58]$ and insect species [59]. The most studied enzymes are those extracted from the mushrooms Mucor rouxii [46, 56, 57], Absidia coerulea [60, 61], and Aspergillus nidulans [62, 63], and two strains of Colletotrichum lindemuthianum $[64,65]$. All enzymes are glycoproteins and are secreted either in the periplasmic region or in the culture medium. In addition, all the enzymes exhibit remarkable thermal stability at their optimum temperature $\left(50^{\circ} \mathrm{C}\right)$ and a very high specificity for the bound $\mathrm{N}$-acetyl-D-glucosamine polymers.

\section{How to follow the transformation reaction of chitin into chitosan (deacetylation) and calculate DD by IR spectroscopy}

The determination of average DD for chitosan may be performed by different techniques: infrared spectroscopy, elementary analysis, potentiometric titration, 
Quantitative Analysis by IR: Determination of Chitin/Chitosan DD

DOI: http://dx.doi.org/10.5772/intechopen.8970 8

\begin{tabular}{|c|c|c|c|c|}
\hline \multirow[t]{2}{*}{ Source } & & $\begin{array}{c}\text { Number of deproteinization } \\
\text { baths }\end{array}$ & $\begin{array}{c}\text { Number of demineralization } \\
\text { baths }\end{array}$ & DA \\
\hline & & $0.3 \mathrm{M} ; \mathrm{NaOH} 80^{\circ} \mathrm{C} ; 1 \mathrm{~h}$ & $0.55 \mathrm{M} \mathrm{HCl} ; 25^{\circ} \mathrm{C} ; 2 \mathrm{~h}$ & \\
\hline Cirripedia & Anatife & 4 & 2 & 51 \\
\hline Reptantia & Red crab & 3 & 5 & 48 \\
\hline Brachyura & Marbled crab & 3 & 3 & 50 \\
\hline Reptantia & Spider crab & 3 & 3 & 47 \\
\hline Macrura & Lobster & 3 & 3 & - \\
\hline \multirow[t]{5}{*}{ Natantia } & Crayfish & 7 & 3 & 51 \\
\hline & Slipper lobster & 3 & 2 & - \\
\hline & $\begin{array}{l}\text { Freshwater } \\
\text { cayfish }\end{array}$ & 3 & 2 & - \\
\hline & Pink shrimp & 3 & 3 & 51 \\
\hline & Gray shrimp & 2 & 2 & 51 \\
\hline Stomatopoda & Squilla & 3 & 3 & 51 \\
\hline Cephalopoda & Squid & 2 & 2 & 51 \\
\hline
\end{tabular}

Table 3.

Comparison of chitosan production from different sources according to Tolaimate et al. [55].

and ${ }^{1} \mathrm{H}$ liquid state and solid state ${ }^{13} \mathrm{C}$-NMR. But FTIR technique has specifically proved to be useful for the analysis of chitin due to its limited solubility in most of the solvents. Nevertheless, FTIR needs a calibration versus an absolute technique like nuclear magnetic resonance (NMR). Identifying the right combination of bands and baselines required a lot of effort, which led the authors to preface a large number of methods in the literature. In fact, several methods have been tried to determine the degree of deacetylation (DD) by FTIR [18, 66-69]. Below is a description of the major three types used to calculate DD by FTIR.

\subsection{Formula 1}

El ouahli [70] calculated from the absorption bands at 1320 and $1420 \mathrm{~cm}^{-1}$. The first band is characteristic of the acetylated amine or amide function, while the second band is chosen as the reference band. The following equation is used to determine DD of chitosan:

$$
\mathrm{DD} \%=100-\left(\frac{\mathrm{A} 1320}{\mathrm{~A} 1420}-0.3822\right) * 1 / 0.03133
$$

\subsection{Formula 2}

This IR characterization formula for chitosan is based on the relationship between the absorbance (A) value of the primary amide at $1655 \mathrm{~cm}^{-1}$ and that of the hydroxyl at $3450 \mathrm{~cm}^{-1}$. The degree of deacetylation (DD) was calculated by Eq. (3), [66, 69]:

$$
\mathrm{DD} \%=100-\left[\left(\frac{\mathrm{A}_{1655}}{\mathrm{~A}_{3450}}\right) * 115\right]
$$




\subsection{Formula 3}

In our study, a ratio had been already proposed $[68,71,72]$. However, choosing an appropriate calculation procedure was not an easy task since the choice of the baseline on FTIR spectra, reference, and the probe bands was difficult. In our work, the average DD was determined by the following formula:

$$
\mathrm{DD} \%=100-\left[\left(\frac{\mathrm{A}_{1655}}{\mathrm{~A}_{3450}}\right) * \frac{100}{1.33}\right]
$$

where $A_{1655}$ is the absorbance at $1655 \mathrm{~cm}^{-1}$ of the amide I band as a measure of the $\mathrm{N}$-acetyl group content and $\mathrm{A}_{3430}$ is the absorbance at $3430 \mathrm{~cm}^{-1}$ due to hydroxyl group as an internal standard. The value 1.33 represents the ratio of this absorbance for a fully acetylated compound. An appropriate baseline in each spectrum was determined by using origin software.

The baseline problem for reference peaks has been studied and summarized in Table 4 and Figure 2. Table 4 shows that there are other formulas for calculating chitosan DD by FTIR and that these formulas have been approved by other techniques such as NMR.

A synthesis of the study on the effect of the operating conditions to know temperature concentration of the base and the time on the reaction of deacetylation will be presented using IR.

\begin{tabular}{lll}
\hline DA calibration curve & Method for DA standard & Ref. \\
\hline 1. $\mathrm{DA}=(\mathrm{A} 1655 / \mathrm{A} 3450) \times 155$ & Titration & {$[66]$} \\
\hline 2. $(\mathrm{A} 1320 / \mathrm{A} 1420)=0.3822+0.03133 \times \mathrm{DA}$ & 1H NMR, 13C NMR & {$[67]$} \\
\hline 3. $(\mathrm{A} 1320 / \mathrm{A} 3450)=0.03146+0.00226 \times \mathrm{DA}$ & 1H NMR, 13C NMR & {$[67]$} \\
\hline 4. $(\mathrm{A} 1560 / \mathrm{A} 2875)=0.2+0.0125 \times \mathrm{DA}$ & Elemental analysis & {$[71]$} \\
\hline
\end{tabular}

Table 4.

Calibration curves from absorption ratios versus standard DA values [73].

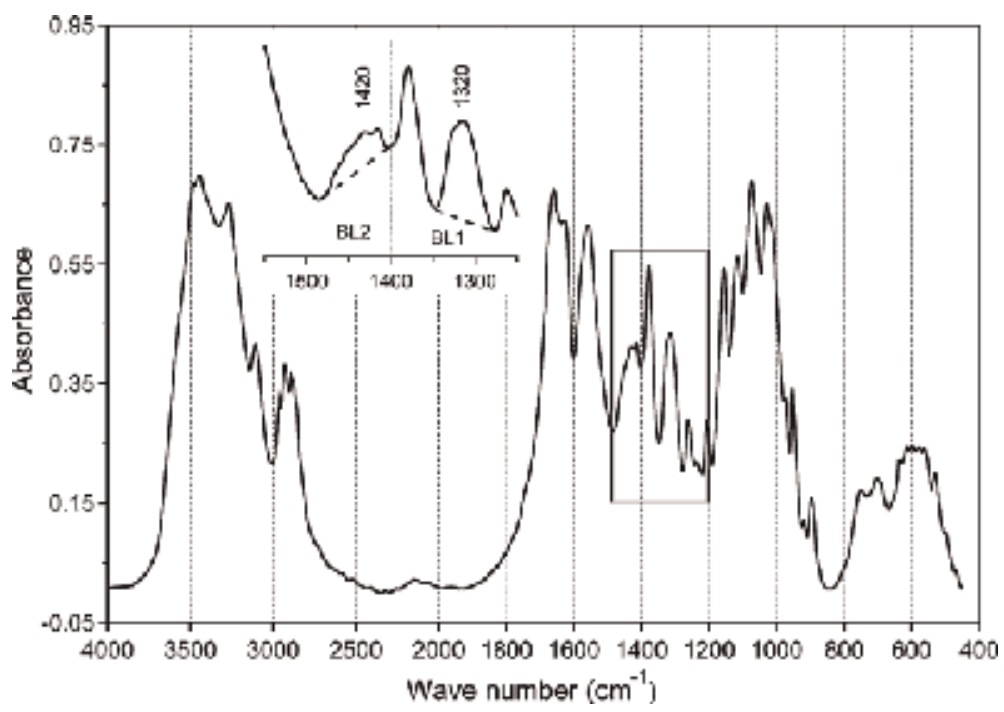

Figure 2.

FTIR spectrum of chitin and the baseline [73]. 


\section{Optimization of chitosan extraction by IR}

\subsection{IR study of the effect of alkaline concentration on the chitin deacetylation}

In this deacetylation reaction, it is an interrelation between the following variables: the concentration of the basic solution, the temperature, and the reaction time. The deacetylation reaction in basic medium is summarized in Eq. (5):

$$
\mathrm{R}-\mathrm{NHCOCH}_{3}+\mathrm{OH}^{-} \rightarrow \mathrm{R}-\mathrm{NH}_{2}+\mathrm{CH}_{3} \mathrm{COO}^{-}
$$

For $\mathrm{C}_{\mathrm{NaOH}}=12 \mathrm{~N}$, the DD values calculated from the FTIR shown in Table 5 do not exceed $55 \%$ and stabilize after a critical time $t<60 \mathrm{~min}$. At a low concentration of $\mathrm{NaOH}$, the equilibrium time became higher, which shows that the chitosan DD is low, which means that the deacetylation reaction is highly dependent on the concentration of $\mathrm{NaOH}$, due to the inaccessibility of acetamide groups in the polymer chain. This type of behavior, observed by other authors, is explained firstly by the fact that the N-deacetylation occurs preferably at the level of the amorphous region of chitin and then passes from the edge to the interior of the crystalline region [26, 27]. The second reason concerns the equilibrium of the reaction and the degradation of chitosan. Other authors $[10,22,28]$ have assumed that it can be controlled by both reaction and diffusion. The low deacetylation of chitin has also been attributed to the rearrangement of acetyl groups in the monomer unit with respect to the $\mathrm{OH}$ hydroxyl group [9]. Analysis of the results of the measurement of the DD (Table 5) shows that this increased by approximately $8 \%(66-74 \% \mathrm{DD})$, at a high concentration of $\mathrm{NaOH}$ $(10 \mathrm{M})$ and with a thermal increase of $20^{\circ} \mathrm{C}\left(100-120^{\circ} \mathrm{C}\right)$. This variation of DD seems less important, only $+2 \%(26-28 \% \mathrm{DD})$ at low concentration of $\mathrm{NaOH}(7.5 \mathrm{M})$. This increase in DD is particularly significant (13\%) with a base concentration increase of $2.5 \mathrm{M}(10 \mathrm{M}$ to $12.5 \mathrm{M})$. Thus, the variation in $\mathrm{NaOH}$ concentration more significantly influences the reaction rate of DD than temperature.

\subsection{IR study of the effect of temperature on the chitin deacetylation}

Table 6 shows the effect of temperature on deacetylation; the more the temperature increases, the more the deacetylation increases, but the total conversion of

\begin{tabular}{|c|c|c|c|}
\hline Temperature of DD & Concentration of the base (M) & Time (mn) & DD \\
\hline \multirow[t]{3}{*}{$25^{\circ} \mathrm{C}$} & 8 & 180 & 38 \\
\hline & 10 & 120 & 45 \\
\hline & 12 & 60 & 55 \\
\hline \multirow[t]{4}{*}{$80^{\circ} \mathrm{C}$} & 4 & 38 & 35 \\
\hline & 8 & 38 & 52 \\
\hline & 10 & 38 & 55 \\
\hline & 12 & 38 & 63 \\
\hline \multirow[t]{4}{*}{$120^{\circ} \mathrm{C}$} & 8 & $50 \mathrm{~min}$ & 40 \\
\hline & 12 & $50 \mathrm{~min}$ & 50 \\
\hline & 8 & $300 \max$ & 65 \\
\hline & 12 & $300 \max$ & 70 \\
\hline
\end{tabular}

Table 5 .

Evolution of $D D$ as a function of the concentration of the base at different temperatures [3]. 


\begin{tabular}{lcccccc}
\hline Ref. [23] & \multicolumn{7}{c}{ Ref. [3] } \\
\hline $\mathbf{T}^{\circ} \mathbf{C} / \mathbf{C}_{\mathbf{N a O H}} \mathbf{~ m o l} / \mathbf{l}$ & $\mathbf{7 . 5}$ & $\mathbf{1 0}$ & $\mathbf{1 2 . 5}$ & $\mathbf{C}_{\text {NaOH }} \mathbf{~ m o l} / \mathbf{l}$ & $\mathbf{T}^{\circ} \mathbf{C}$ & DD \\
\hline 40 & - & 1 & 2 & 8 & 180 & 38 \\
\hline 60 & 10 & 14 & 20 & 10 & 120 & 45 \\
\hline 80 & 24 & 60 & 67 & 12 & 60 & 55 \\
\hline 100 & 26 & 68 & 78 & 8 & 180 & 38 \\
\hline 120 & 28 & 74 & 90 & 10 & 120 & 45 \\
\hline
\end{tabular}

Table 6.

Evolution of $D D$ in relation to temperature at different concentrations of the base [3, 23].

chitin to chitosan is only carried out at the temperature of $80^{\circ} \mathrm{C}$ and for high concentrations of the base [23]. Analysis of the results of the measurement of DD shows that it has increased approximately $8 \%(66-74 \% \mathrm{DD})$, at high concentration of $\mathrm{NaOH}(10 \mathrm{M})$ and a thermal increase of $20^{\circ} \mathrm{C}\left(100-120^{\circ} \mathrm{C}\right)$. This variation of DD seems less important, only $+2 \%(26-28 \% \mathrm{DD})$ at low concentration of $\mathrm{NaOH}$ $(7.5 \mathrm{M})$. This discrepancy can be considered as an error of analysis. However, this increase in DD is particularly significant (13\%) with a base concentration increase of $2.5 \mathrm{M}(10-12.5 \mathrm{M})$. The temperature in this step is a factor that weakens the binding of the acetyl groups and accelerates the deacetylation reaction. Ahlafi and al [3] also found that the DDs increase as the $\mathrm{NaOH}$ concentration and temperature increase as the time of reaction increases. These reached a maximum of $63 \%$ and $71 \%$ for $\mathrm{C}_{\mathrm{NaOH}}=12 \mathrm{~N}$ at $\mathrm{T}=80$ and $120^{\circ} \mathrm{C}$, respectively. On the other hand, it can be observed that the critical time decreases when the temperature and the concentration of $\mathrm{NaOH}$ increase. It can be seen in the case of $\mathrm{C}_{\mathrm{NaOH}}=12 \mathrm{~N}$ and $\mathrm{T}=120^{\circ} \mathrm{C}$. The increase of DD values with these parameters can be attributed to the change of the structure during deacetylation of chitin at height temperature, as confirmed by FTIR studies. These results confirm the hypothesis that the interaction of concentration and thermal energy are the main criteria to be taken into account for the deacetylation reaction.

\subsection{FTIR study of the effect of time on the chitin deacetylation}

The analysis of the results (Table 7) shows that the best deacetylation started after $60 \mathrm{~min}$, for the three concentrations of $\mathrm{NaOH}$ studied. At this time, the activation energy for deacetylation can be reached and the chemical bond break occurs. However, this breakdown of the acetyl groups is significant only at the level of the high alkaline concentration (> $10 \mathrm{M})$. Diffusion of the alkaline solution on the shell substrate increases with time. These results confirm the hypothesis that there is an interaction between the concentration of $\mathrm{NaOH}$ and the reaction time on DD.

At the lowest $\mathrm{NaOH}$ concentration $(7.5 \mathrm{M})$, the percentage of deacetylation increases from about $60^{\circ} \mathrm{C}$ but remains low even at $120^{\circ} \mathrm{C}$ (Table 7). The extracted product remains predominantly in the form of chitin. The deacetylation is terminated after a period ranging from 90 to $120 \mathrm{~min}$ depending on the concentration of $\mathrm{NaOH}$. Moreover, for a lower concentration of $\mathrm{NaOH}(8.75 \mathrm{M})$, this reaction is incomplete even after $3 \mathrm{~h}$ for different authors [74]. Therefore, in the case of a low concentration of $\mathrm{NaOH}$ (less than $10 \mathrm{M}$ ), the deacetylation does not occur completely. Thus, to obtain a good-quality chitosan, it is necessary to resume the treatment several times using $\mathrm{NaOH}$ solution $[75,76]$. This reprocessing scheme is technically not easy and economically unviable on a large scale. So, for better deacetylation, $1 \mathrm{~h}$ is sufficient for concentrations greater than $10 \mathrm{M}$. 


\begin{tabular}{lccccccc}
\hline & \multicolumn{2}{c}{$[\mathbf{2 3}]$} & \multicolumn{3}{c}{$\mathbf{1 2 0}{ }^{\circ} \mathrm{C}[3]$} \\
\hline $\mathbf{C ~} \mathbf{~ m o l} / \mathbf{l} / \mathbf{t ~ m n}$ & $\mathbf{8 . 7 5}$ & $\mathbf{1 0}$ & $\mathbf{1 1 . 2 5}$ & $\mathbf{1 2 . 5}$ & $\mathbf{C ~} \mathbf{~ m o l} / \mathbf{l} / \mathbf{t ~ m n}$ & $\mathbf{8}$ & $\mathbf{1 2}$ \\
\hline 30 & 8 & 23 & 42 & 45 & 50 & 40 & 50 \\
\hline 60 & 13 & 60 & 70 & 78 & 100 & 48 & 52.5 \\
\hline 90 & 18 & 75 & 81 & 86 & 150 & 53 & 54 \\
\hline 120 & 23 & 82 & 86 & 91 & 200 & 55 & 67 \\
\hline 150 & 26.5 & 84 & 88 & 92 & 250 & 64 & 68 \\
\hline 180 & 30 & 86 & 90 & 93 & 300 & 65 & 70 \\
\hline
\end{tabular}

Table 7 .

Evolution of $D D$ as a function of time at different concentrations of the base [3, 23].

\begin{tabular}{lccc}
\hline $\mathbf{T}\left({ }^{\circ} \mathbf{C}\right)$ & 25 & 80 & 120 \\
\hline $\mathrm{k}_{2}\left(\mathrm{~min}^{-1}\right)$ 2nd step & $1.70 \times 10^{-5}$ & $2.23 \times 10^{-4}$ & $22 \times 10^{-4}$ \\
\hline
\end{tabular}

Table 8 .

Apparent rate constant [3].

The rate of deacetylation therefore depends not only on the concentration of $\mathrm{NaOH}$ used but also on the temperature for a breakdown of the acetyl bonds. However, a high temperature leads to a degradation of chitosan, which causes the viscosity $\eta$ to drop and the molecular weight $M(\eta=\mathrm{kM}$ a) to drop, which affects the solubility of chitosan. The variation of the concentration of $\mathrm{NaOH}$ does not affect its molecular weight [76].

\subsection{Determination of apparent rate constant $k$ and energetic activation (Ea)}

The chitin deacetylation process followed the pseudo-first-order kinetics for all the temperatures studied $\left(25,80\right.$, and $\left.120^{\circ} \mathrm{C}\right)$ and at the same alkaline concentration $(12 \mathrm{~N})$ [3]. The Table 8 showed that the values decreased as a function of temperature. This indicates that the speed of the deacetylation reaction is faster at the beginning of the reaction $t<60 \mathrm{~min}$, but it was very slow at the end of reaction.

The apparent activation energy was estimated at about $48.76 \mathrm{~kJ} / \mathrm{mol}$ from the straight line of the Arrhenius plot (in k vs. (1/T)) [3]. This value is in the same order of magnitude as that found by other authors for heterogeneous $\mathrm{N}$-deacetylation performed between 80 and $120^{\circ} \mathrm{C}[10,12,22,26]$. The concentration of $\mathrm{NaOH}$ significantly influences the variation of the reaction temperature. Rinaud and al. [14] mention that at a $\mathrm{NaOH}$ concentration of $10-15 \mathrm{M}$, the energetic activation (Ea) of the deacetylation is, respectively, about $22-50 \mathrm{~kJ} / \mathrm{mol}$, which makes it possible to increase the degree of deacetylation. The results indicated that the reaction at higher concentration and temperature proceeded easier than that at their lower values.

\subsection{Mechanism of the chitin deacetylation}

The reagent diffusion mechanism represents the second step in deacetylation of chitin. Recently, Sarhan et al. [28] have proposed a mechanism in which heterogeneous $\mathrm{N}$-deacetylation is controlled by both reaction and diffusion: the first step involves the reaction of the onium salt, designated $\left(\mathrm{Q}^{+} \mathrm{X}^{-}\right)$, with $\mathrm{NaOH}$ to give the 
corresponding onium hydroxide $\left(\mathrm{Q}^{+} \mathrm{OH}^{-}\right)$capable of diffusing from the aqueous phase to the organic phase to start the deacetylation process by attacking the $\mathrm{C}=\mathrm{O}$ of the acetyl group and then at the end of the reaction of hydrolysis, the resulting onium acetate $\left(\mathrm{CH} 3 \mathrm{COO}^{-} \mathrm{Q}^{+}\right)$will diffuse into the aqueous phase to be regenerated to a new onium hydroxide by the reaction with $\mathrm{NaOH}$. From our results, the DD/ $\mathrm{C}_{\mathrm{NaOH}}$ ratios remain constant at each temperature at longer deacetylation times, which means that the deacetylation reaction is complete.

\section{IR statistic study for optimization of deacetylation conditions}

The results obtained under various conditions were analyzed statistically using multilinear regression analysis (uncertainty value $=0.05$ ) [23]. The $\mathrm{NaOH}$ concentration, temperature, and reaction time were chosen as independent parameters for the three-variable and three-level (maximum, mean, and minimum) factorial design. The best equation obtained for the chitosan extraction process in this study is Eq. (6). Shrimp at a higher temperature is not reliable. In addition, the production of chitosan at high temperature causes degradation of the container and therefore involves manipulations more difficult to adapt for industry. To ensure the quality of the chitosan product, the digestion temperature must be kept constant at $110^{\circ} \mathrm{C}$.

$$
\begin{aligned}
\% \mathrm{DD}= & 72,56+5,6 *(\mathrm{NaOH})+3,78 *(\text { temperature })+24,86 *(\text { temps }) \\
& -11,92 *(\text { temps })
\end{aligned}
$$

The values of this equation indicate that the effect of three factors studied influences DD in the following order: reaction time $(24.86)>\mathrm{NaOH}$ concentration (5.6) > reaction temperature (3.78). Further, a reaction time of $152 \mathrm{~min}(\approx 2.5 \mathrm{~h})$ is predicted from Eq. (5) to reach $90 \% \mathrm{DD}$ using a $\mathrm{NaOH}$ concentration of $12.5 \mathrm{M}$ and a temperature set at $110^{\circ} \mathrm{C}$. This time predicted by the factorial plane seems consistent with the actual deacetylation reaction time $(120 \mathrm{~min})$ with an error of $27 \%$.

The production of chitosan from dried shrimp exoskeletons can be done in 1 day instead of the 3 days required by the conventional method. The results obtained show that the extracted chitosan has a DD greater than $90 \%$ under optimal conditions by the hydrothermal-chemical technique in two stages at $110^{\circ} \mathrm{C}$, that is, 11.25 M NaOH for $3 \mathrm{~h}$ or $12.5 \mathrm{M} \mathrm{NaOH}$ during $2 \mathrm{~h}$. For the extraction of chitosan at an SD of $85 \%$ in the context of water treatment, the transformation procedure must be carried out under the following optimal conditions:

- Demineralization at $50^{\circ} \mathrm{C}$ for $2.5 \mathrm{~h}$ in $2 \mathrm{M} \mathrm{HCl}$

- Deproteinization and deacetylation at $110^{\circ} \mathrm{C}$ with $11.25 \mathrm{M} \mathrm{NaOH}$ for $2 \mathrm{~h}$

\section{Deacetylation of chitin by compression method study by FTIR}

A new method of producing very high DD content chitosan under low concentration alkaline conditions has been introduced by Xiaofei et al. [79]. The synthesis was produced at high temperatures and pressures. The results in Table 9 were deduced from IR spectra. Compared to traditional methods, low concentration alkaline and short reaction time are excellent benefits. In addition, compared to the enzymatic and organic solvent treatment method, the pressure method was inexpensive and convenient without further purification. Excellent repeatability and simplified operation increased its availability in production and large application 


\begin{tabular}{|c|c|c|c|c|c|}
\hline $\mathrm{C} / \%$ & $\mathrm{CT}: \mathrm{NaOH}$ & $\mathrm{RT} / \mathrm{min}$ & $\mathrm{T} / \mathrm{C}$ & Productivity/\% & $\mathrm{DD} / \%$ \\
\hline 5 & $1: 20$ & 120 & 120 & 88.95 & A \\
\hline 5 & $1: 20$ & 240 & 120 & 90.48 & A \\
\hline 10 & $1: 20$ & 120 & 120 & 88.41 & A \\
\hline 10 & $1: 20$ & 240 & 120 & 90.88 & A \\
\hline 15 & $1: 20$ & 120 & 120 & 86.76 & A \\
\hline 15 & $1: 20$ & 240 & 120 & 88.73 & A \\
\hline 20 & $1: 20$ & 120 & 120 & 87.66 & A \\
\hline 20 & $1: 20$ & 240 & 120 & 86.94 & A \\
\hline 25 & $1: 20$ & 120 & 120 & 85.69 & A \\
\hline 25 & $1: 20$ & 240 & 120 & 85.88 & A \\
\hline 30 & $1: 20$ & 120 & 120 & 85.19 & A \\
\hline 30 & $1: 20$ & 240 & 120 & 86.53 & A \\
\hline 30 & $1: 20$ & 360 & 120 & 85.44 & A \\
\hline 35 & $1: 20$ & 120 & 120 & 84.17 & 85.05 \\
\hline 40 & 1:20 & 120 & 120 & 82.00 & 90.13 \\
\hline 40 & $1: 20$ & 120 & 120 & 81.27 & 94.26 \\
\hline 40 & 1:20 & 120 & 120 & 81.93 & 89.19 \\
\hline
\end{tabular}

Table 9.

The effect of different reaction conditions on DD value of chitosan [79].

chitosan scale with large quantity production. In order to produce chitosan with a very high content of DD by chitin in a more efficient and more environmentally friendly way, Xiaofei et al. [79] have changed the pressure and have used the multistep method for the deacetylation of chitin in alkaline at low concentration. They adopted the alkaline recycling model using alkaline waste in the next deacetylated step. In this model, they added an alkali according to the different demands of each phase and eliminated acetyl in a timely manner, in case the high concentration of the acetyl group would inhibit the deacetylation. In addition, the use of the base in several steps will be effective not only to control the degree of deacetylation but also to control the molecular weight of the resulting product.

Working under high pressure and with low concentrations of the base, they were able to extract chitosan with DD of $100 \%$. In fact, only $15 \%$ of alkali solution and a ratio of 1:10 chitosan powder to $\mathrm{NaOH}$ solution and to a pressure of less than $0.11-0.12 \mathrm{MPa}$ for $120 \mathrm{~min}$ lead to $100 \% \mathrm{DD}$. When the alkali concentration varies from $5-15 \%$, the very high value DD chitosan (up to 95\%) is produced. The method under pressure to prepare $100 \%$ deacetylated chitosan with less environmental pollution is very interesting.

\section{Deacetylation of chitin under microwave irradiation effect}

To extract a large quantity of chitosan, industrialists need high temperatures and chemicals in large quantities. In addition, the conventional process requires a lot of time and consumes a lot of energy, which would harm the environment. Recently, microwave irradiation has been used as an unconventional energy source in chemical reactions. The objective of this study is to synthesis chitosan under microwave 


\begin{tabular}{lcccc}
\hline Chitin treatment & NaOH conc. (\%) & DD\% & Mw (k Daltons) & Solubility (\%) \\
\hline \multirow{2}{*}{20 mesh } & 30 & $67.58^{\mathrm{F}} \pm 0.92$ & 2415.09 & $66.31^{\mathrm{H}} \pm 0.35$ \\
\cline { 2 - 4 } & 40 & $75.77^{\mathrm{E}} \pm 3.54$ & 1476.21 & $74.94^{\mathrm{G}} \pm 0.79$ \\
\cline { 2 - 5 } & 50 & $78.83^{\mathrm{D}} \pm 1.05$ & 1267.11 & $96.77^{\mathrm{BC}} \pm 0.17$ \\
\hline \multirow{2}{*}{40 mesh } & 30 & $76.89^{\mathrm{DE}} \pm 0.89$ & 866.03 & $99.05^{\mathrm{A}} \pm 0.05$ \\
\cline { 2 - 5 } & 40 & $78.64^{\mathrm{D}} \pm 0.86$ & 1107.50 & $92.79^{\mathrm{D}} \pm 0.01$ \\
\hline \multirow{2}{*}{60 mesh } & 50 & $83.05^{\mathrm{C}} \pm 0.29$ & 2160.88 & $95.60^{\mathrm{C}} \pm 0.87$ \\
\cline { 2 - 5 } & 30 & $88.39^{\mathrm{B}} \pm 0.49$ & 949.95 & $83.28^{\mathrm{F}} \pm 0.87$ \\
\cline { 2 - 5 } & 40 & $89.17^{\mathrm{B}} \pm 0.28$ & 1274.85 & $85.57^{\mathrm{E}} \pm 1.37$ \\
\hline Commercial chitosan & 50 & $95.19^{\mathrm{A}} \pm 0.74$ & 4467.05 & $97.73^{\mathrm{AB}} \pm 0.95$ \\
\hline & & & \\
\hline
\end{tabular}

Data are the mean.

Mean values in the same column bearing the same superscript do not differ significantly.

Table 10.

Degree of deacetylation (DD), molecular weight ( $m w)$, and solubility of chitosan samples extracted from shrimp wastes using microwave technique [81].

irradiation in order to reduce the impact of environmental pollution due to excessive use of chemical treatments [80-82]. The study will examine the effect of chemical addition, reaction time, operating temperature on manufacturing, and chitosan DD under microwave irradiation. These results will be compared to those from conventional heating methods to compare results. As part of this research, they developed the design and manufacture of a proton prototype for the production of chitosan from shrimp shell waste. Research has concluded that microwaves will accelerate reaction time.

The results showed that the demineralization condition of shrimp waste was achieved at the concentration of $\mathrm{HCl} 3,5 \mathrm{~N}$ solution with the weight ratio of shrimp shell waste and $\mathrm{HCl}$ solution of $1: 5(\mathrm{w} / \mathrm{v})$, at a temperature of $50^{\circ} \mathrm{C}$ during $1 \mathrm{~h}$ heating. In those conditions, the ash content was $8.06 \%$. Ash content decreases to $5.4 \%$ if the demineralization reaction is carried out under microwave irradiation with 130 watts for $10 \mathrm{~min}$. The optimum condition of the deproteinization process was achieved by heating at a temperature of $70^{\circ} \mathrm{C}$ for $2 \mathrm{~h}$ and at $4 \% \mathrm{NaOH}$ concentration for shrimp waste ratio: a NaOH solution of 1:5 (w/v). In this condition, they obtained nitrogen levels of $1.882 \%$ (11.763\% protein content). If the deproteinization reaction was performed under microwave irradiation with $130 \mathrm{~W}$ of power for $15 \mathrm{~min}$, the nitrogen content obtained was $1.833 \%$ (11.461\% protein content) (Table 10).

\section{Comparison between IR and other techniques for calculating DD}

To differentiate chitin from chitosan, it is necessary to define the degree of acetylation (DA), that is to say the ratio of the number of units comprising an acetyl group on the number of units in the molecule. We can also speak of degree of deacetylation (DD) such that: DD = 100 - DA in\%.

The calculation of DD was made according to several methods described in the literature: acid and basic conductometric method, $\mathrm{pH}$-metric method, UV method, and IR spectroscopy method. In a recent study [70], an attempt was made to 
compare IR and other analytical methods to compute DD. The analysis was performed by acidic conductometric assay. Basic conductometric dosing and $\mathrm{pH}$ metric dosing techniques will be compared to IR.

\subsection{Determination of the degree of deacetylation by conductometric assay}

According to $\mathrm{Yu}$ et al. [22], the conductometric assay is an adequate and accurate method to determine the degree of deacetylation of chitosan. It was carried out in basic and acid medium.

\subsubsection{Basic conductivity measurement}

A solution of chitosan was prepared by dissolving a mass of $150 \mathrm{mg}$ of chitosan in $10 \mathrm{ml}$ of hydrochloric acid $(0.1 \mathrm{~N})$ and then the volume was adjusted to $200 \mathrm{ml}$ by addition of distilled water. The prepared solution is titrated with stirring with sodium hydroxide solution $(0.1 \mathrm{~N})$. Figure $3 \mathrm{~A}$ shows the change in the volume of sodium hydroxide as a function of the conductivity of the chitosan solution. The curve has two points of inflection. The difference in the volume of $\mathrm{NaOH}$ between these two points corresponds to the amount of $\mathrm{HCl}$ required to dissolve the chitosan, which is to say to transform the $-\mathrm{NH}_{2}$ groups into $-\mathrm{NH}_{3}{ }^{+}$.

The degree of deacetylation (DD) of chitosan is then determined from the following relationship [70]:

$$
\mathrm{DD}=203 \times(\mathrm{V} 2-\mathrm{V} 1) \times \frac{\mathrm{N}}{\mathrm{m}+42 \times(\mathrm{V} 2-\mathrm{V} 1) \times \mathrm{N}} \times 100
$$

where $\mathrm{N}$ is the normality of the $\mathrm{NaOH}$ solution (mol/l); V2 and $\mathrm{V} 1$ are the equivalent volumes of $\mathrm{NaOH}$ representing two inflection points, respectively; $\mathrm{M}$ is the mass of chitosan; $203(\mathrm{~g} / \mathrm{mol})$ is the molar mass of the acetyl monomer; and 42 $(\mathrm{g} / \mathrm{mol})$ is the difference between the molecular weight of the acetyl monomer and the molecular weight of the deacetylated monomer.

$$
\mathrm{DD}=203 \times(9.4-2.7) .10-3 \times \frac{0.1}{0.15+42 \times(9.4-2.7) .10-3 \times 0.1} \times 100
$$

The degree of deacetylation according to the conductometric method is: $\mathrm{DD}=76.35 \%$.
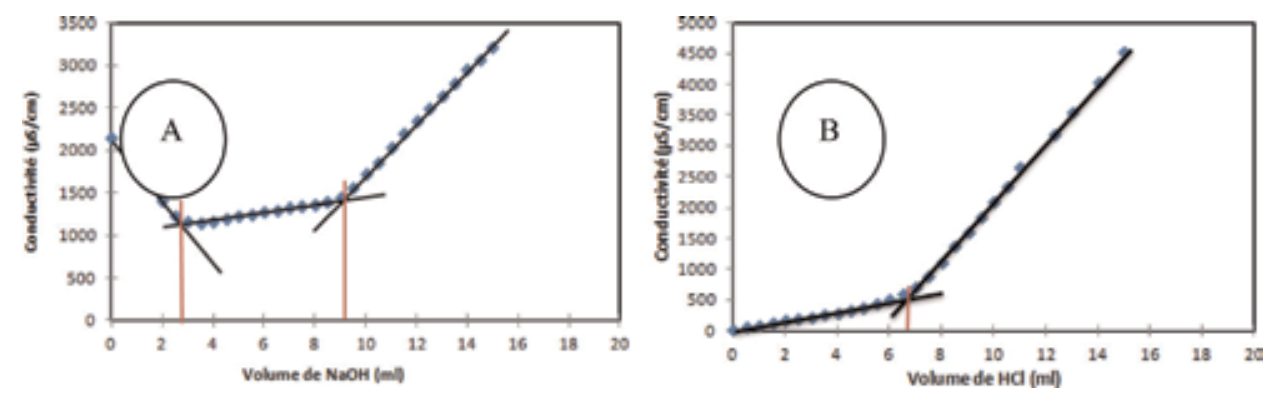

Figure 3.

(A) Variation of the conductivity of the chitosan solution as a function of the volume of the base (B) variation of the conductivity of the chitosan solution as a function of the volume of the acid solution. 


\subsubsection{Acidic conductivity dosing}

The determination of the DD by acid conductometric assay is carried out as follows: a mass of $150 \mathrm{mg}$ of chitosan is dispersed in $200 \mathrm{ml}$ of distilled water; while stirring, the mixture is titrated with $0.1 \mathrm{~N} \mathrm{HCl}$ solution. Figure 3B shows the evolution of the conductivity of the chitosan solution as a function of the volume of $\mathrm{HCl}$ poured. The point of inflection corresponds to the amount of $\mathrm{HCl}$ consumed by the amine groups of chitosan. The DD will be calculated from the following equation:

$$
\mathrm{DD}=(203 \times \mathrm{V} \times \mathrm{N}) /(\mathrm{m}+42 \times \mathrm{V} \times \mathrm{N}) \times 100
$$

where $\mathrm{N}$ is the normality of the $\mathrm{HCI}$ solution (mol/l), $\mathrm{V}$ is the volume corresponding to the inflection point as shown in Figure 3B, $\mathrm{m}$ is the mass of chitosan $(\mathrm{g})$, and $42(\mathrm{~g} / \mathrm{mol})$ is the difference between the molecular weight of the acetylated monomer and the molecular weight of the deacetylated monomer.

DDA calculation gives:

$$
\begin{aligned}
\mathrm{DD}= & (203 \times 6.8 \times 10-3 \times 0.1) /(0.15+42 \times 6.8 \times 10-3 \times 0.1) \times 100 \\
& \mathrm{DD}=77.30 \% .
\end{aligned}
$$

\subsection{Determination of the degree of deacetylation by $\mathrm{pH}$-metric determination}

The determination of the DD by $\mathrm{pH}$-metric assay was carried out according to the method described in the literature [77, 78].

A solution of chitosan was prepared by dissolving a mass of $125 \mathrm{mg}$ of chitosan in a solution of excess $\mathrm{HCl}(0.1 \mathrm{~N})$ and then neutralizing this solution with sodium hydroxide solution $(0.05 \mathrm{~N})$. Figure $4 \mathrm{~A}$ shows the titration curve of chitosan, and Figure 4B shows the corresponding secondary derivative. From this last curve, the amount of hydrochloric acid necessary to protonate the amine groups is determined. The degree of deacetylation calculated from this method is $77.10 \%$.

The results of the DDA obtained by the different assay methods are shown in

\section{Table 11.}

The average value of DD calculated for the chitosan prepared during this work is $77.32 \%$. So, IR spectroscopy remains the simplest and most economical technique for calculating DD.

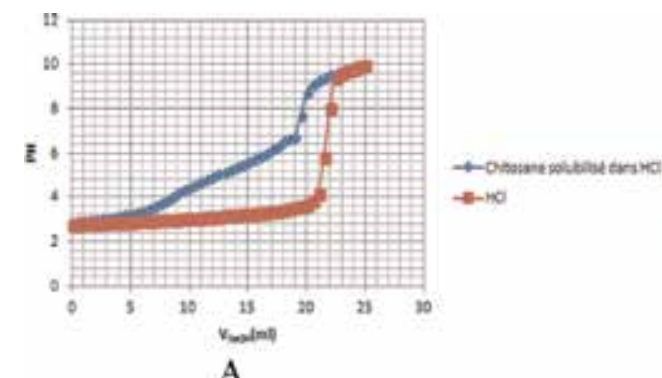

Figure 4.

(A) Variation of the $\mathrm{pH}$ according to the volume of soda poured for the solution of the chitosan and the solution of the hydrochloric acid, (B) secondary derivative curve corresponding to the assay. 
Quantitative Analysis by IR: Determination of Chitin/Chitosan DD

DOI: http://dx.doi.org/10.5772/intechopen.8970 8

\begin{tabular}{lc}
\hline Dosing method & DDA (\%) \\
\hline Basic conductivity meter & 76.35 \\
\hline Conductometric acid & 77.30 \\
\hline pH-metric & 77.10 \\
\hline Infrared spectroscopy & 78.50 \\
\hline Calculated average & 77.32 \\
\hline
\end{tabular}

Table 11.

DD values obtained with different assay methods [70].

\begin{tabular}{lccccc}
\hline Method & $\mathbf{1}(\mathbf{\%})$ & $\mathbf{2}(\mathbf{\%})$ & $\mathbf{3}(\mathbf{\%})$ & $\mathbf{4}(\mathbf{\%})$ & $\mathbf{5}(\mathbf{\%})$ \\
\hline DDTitration1 & 90.82 & 90.57 & 94.93 & 93.04 & 92.48 \\
\hline DDTitration2 & 92.85 & 94.62 & 93.97 & 95.40 & 94.99 \\
\hline DDTitration3 & 93.10 & 94.45 & 94.86 & 94.35 & 94.66 \\
\hline DD-UV1 & 95.97 & 95.96 & 99.42 & 98.18 & 99.34 \\
\hline DD-UV2 & 94.44 & 94.87 & 97.72 & 97.07 & 97.00 \\
\hline DD-UV3 & 95.02 & 95.36 & 98.07 & 97.42 & 97.99 \\
\hline & & Part-II & & \\
\hline DD-UV1 & 82.00 & 95.96 & 97.68 & 100.00 & 100.00 \\
\hline DD-UV2 & 82.27 & 95.86 & 97.73 & 100.00 & 100.00 \\
\hline DD-IR1658/3450 & 80.43 & 94.50 & 94.60 & 95.37 & 96.33 \\
\hline DD-IR1320/1420 & 87.64 & 100.00 & 100.00 & 100.00 & 100.00 \\
\hline
\end{tabular}

Table 12.

Comparison of DD value measured by different methods [79].

\subsection{Comparison to UV: visible}

Another study presented by Xiaofei and al [79] gave a comparison between IR spectroscopy and other techniques and showed the importance of FTIR for calculating DD (Table 12). They have been measured for 3 or 2 times.

\section{Conclusion}

The calculation of DD and the number of amine present in chitosan by FTIR allows first to follow the transformation reaction of chitin into chitosan and others by finding the optimal conditions for the synthesis of chitosan by studying the effect of different parameters, namely the concentration of the base, the temperature, and the duration of the reaction. The valorization of shrimp exoskeletons by extraction of chitosan according to the hydrothermal-chemical technique proposed in two stages makes it possible to reduce the production time by at least four times compared to the conventional technique in three stages (3-4 days). In addition, the consumption of digestion and energy chemicals is also significantly reduced. The chitosan obtained by the two-step technique is of good quality. Indeed, the degree 
of deacetylation is greater than $90 \%$ under the optimal conditions for the simultaneous deproteinization and deacetylation.

The compression method for preparing $100 \%$ deacetylated chitosan with less environmental pollution was studied by FTIR. The $100 \%$ fully deacetylated chitosan was produced in low-concentration alkali and high-pressure conditions, which only requires $15 \%$ alkali solution and 1:10 chitosan powder to $\mathrm{NaOH}$ solution ratio under 0.11-0.12 MPa for $120 \mathrm{~min}$. When the alkali concentration varied from 5-15\%, the chitosan with ultra-high DD value (up to 95\%) is produced.

In parallel, the FTIR calculation was also used to show that the microwave could be used in the extraction step of chitosan from chitin. From these results, it could be concluded that shrimp waste is an excellent source for chitin, and the yields of chitosan increased with decreasing the chitin particle size and increasing the concentration of $\mathrm{NaOH}$ solution used in deacetylation step. The highest degree of deacetylation was obtained from chitin samples at particle size of 60 mesh deacetylated by $50 \% \mathrm{NaOH}$ solution, and it was $95.19 \%$ compared with $85 \%$ for commercial chitosan. Based on this synthesis, it is concluded that FTIR is an effective and reliable technique for the determination of DD and the study of the deacetylation reaction of chitin. All the formulas quoted are valid for calculating DD of chitosan, but the most reliable formula and the formula most approved by other techniques is formula 3 quoted in our works.

\title{
Author details
}

\author{
Boukhlifi Fatima \\ Equip, Materials and Applied Catalysis (MCA), Faculty of Science, Moulay Ismail \\ University, Meknes, Morocco
}

*Address all correspondence to: boukhlifi1@yahoo.fr

\section{IntechOpen}

(C) 2020 The Author(s). Licensee IntechOpen. This chapter is distributed under the terms of the Creative Commons Attribution License (http://creativecommons.org/licenses/ by/3.0), which permits unrestricted use, distribution, and reproduction in any medium, provided the original work is properly cited. (c) BY 


\section{References}

[1] Okazaki S, Tachibana T,

Naganuma A, Mano N, Kuge S.

Multistep disulfide bond formation in

Yap1 is required for sensing and transduction of $\mathrm{H}_{2} \mathrm{O}_{2}$ stress signal.

Molecular Cell. 2007;27(4):675-688

[2] Boukhlifi F, Mamouni FZ, Razouk R. Chitin/Chitosan's bio-fertilizer: Ch 16: Usage in vegetative growth of wheat a nd potato crops. In: Dongre RS, editor. Chitin-Chitosan - Myriad Functionalitie $\mathrm{s}$ in Science and Technology. Rijeka: IntechOpen; 2018. p. 75208

[3] Ahlafi H, Moussout H, Boukhlifi F, Echetna M, Naciri Bennani M, Slimane SM. Kinetics of N-deacetylation of chitin extracted from shrimp shells collected from coastal area of Morocco. Mediterranean Journal of Chemistry. 2013;2(3):503-513

[4] Ouattara B, Simard RE, Piette G, Begin A, Holley RA. Inhibition of surface spoilage bacteria in processed meats by application of antimicrobial films prepared with chitosan. International Journal of Food Microbiology. 2000;62:139-148

[5] Muzzarelli RAA, Muzzarelli C. Chitosan chemistry: Relevance to the biomedical sciences. Advances in Polymer Science Journal. 2005;186: 151-209

[6] Alves MN, Mano JF. Chitosan derivatives obtained by chemical modifications for biomedical and environmental applications. International Journal of Biological Macromolecules. 2008;43:401-414

[7] Dzung NA. Chitosan and chitosan derivatives as potential adjuvants for influenza vaccine. In: Kim SK, editor. Chitin and Chitosan Derivatives: Advances in Drug and Discovery and Developments. CRC Taylors \& Francis; 2014
[8] Ngo DN, Kim SK. Antioxidant, antimicrobial properties of chitin, chitosan, and their derivatives. Advances in Food and Nutrition Research. 2014;73:15-31

[9] Boukhlifi F, Bencheikh A. Study of the competitive adsorption of heavy metals on crude chitin: Application to wastewater from a chemical industry. The Water Tribune. 2001;55(611/3): 37-43

[10] Boukhlifi F, Bencheikh A.

Characterization of natural biosorbents used for the depollution of waste water. Annales de Chimie Science des Materiaux. 2000;256:153-160

[11] Boukhlifi F. Study of the retention of metallic micro-pollutants $(\mathrm{Pb}, \mathrm{Cd}, \mathrm{Cu}$ and $\mathrm{Zn}$ ) on new biosorbent materials: liquid industrial effluent purification tests [doctoral thesis]. Morocco: Chouaib Doukkali El University jadida; 2000

[12] Boukhlifi F, El Akili C, Moussout H, Benzakour A, Ahlafi H. Treatment of global rejection of electroplating industry by raw chitin. International Journal of Applied Environmental Sciences. 2013;8:13-23

[13] Boukhlifi F, Bencheikh A, Ahlafi H. Characterization and adsorption of chitin toward copper $\mathrm{Cu}^{2+}$. Physical and Chemical News. 2011;58:67-72

[14] Rinaudo M, Milas M, Ledung P.

Characterization of chitosan-Influence of ionic-strength and degree of acetylation on chain expansion. International Journal of Biological Macromolecules. 1993;15(5):281-285

[15] Wei W, Bo SQ, Li SQ, Wen Q. Determination of the mark-Houwink equation for chitosans with different degrees of desacetylation. International 
Journal of Biological Macromolecules. 1991;13(5):281-285

[16] Dash M, Chiellini F, Fernandez EG, Piras AM. Statistical approach to the spectroscopic determination of the desacetylation degree of chitins and chitosans. Carbohydrate Polymers. 2011;86:65-71

[17] Chatelet O, Damour A, Domard A. Influence of the degree of acetylation on some biological properties of chitosan films. Biomaterials. 2001;22:261-268

[18] Domszy JG, Roberts GAF. Evaluation of infrared spectroscopic techniques for analyzing chitosan. Makromolekulare ChemieMacromolecular Chemistry and Physics. 1985;186(8):1671-1677

[19] Sannan T, Kurita K, Ogura K, Iwakura Y. Studies on chitin. 7. Infrared spectroscopic determination of degree of desacetylation. Polymer. 1978;19(4): 458-459

[20] Muzzarelli RAA, Rocchetti R. Determination of the degree of acetylation of chitosans by 1st derivative ultraviolet spectrophotometry. Carbohydrate Polymers. 1985;5(6): 461-472

[21] Alonso IG, Peniche-Covas C, Nieto JM. Determination of the degree of acetylation of chitin and chitosan by thermal-analysis. Journal of Thermal Analysis. 1983;28(1):189-193

[22] Yu JH, YM D, Zheng H. Blend films of chitosan/gelatin. Journal of Wuhan University (Natural Sciences Edition). 1999;45:440-444

[23] Truong TO, Hausler R, Monette F, Niquette $\mathrm{P}$. Valorisation des résidus industriels de pêches pour la transformation de chitosane par technique hydrothermochimique. Revue des Sciences de l'Eau. 2007;20(3): 253-262
[24] Horst MN, Walker AN, Klar E. The pathway of crustacean chitin synthesis. In: Horst MN, Freeman JA, editors. The Crustacean Integument: Morphology and Biochemistry. Boca Raton, FL: CRC, USA; 1993. pp. 113-149

[25] Younes I, Rinaudo M. Review chitin and chitosan preparation from marine sources. Structure, properties and applications. Marine Drugs. 2015;13: 1133-1174

[26] No HK, Hur EY. Control of foam formation by antifoam during demineralization of crustacean shell in preparation of chitin. Journal of Agricultural and Food Chemistry. 1998; 46:3844-3846

[27] Percot A, Viton C, Domard A. Characterization of shrimp shell deproteinization. Biomacromolecules. 2003;4:1380-1385

[28] Muzzarelli RAA, Tanfani F, Emanuelli M, Gentile S. The chelation of cupric ions by chitosan membranes [Callinectes sapidus, blue crab shell]. Journal of Applied Biochemistry. 1980; 2:380-389

[29] Moorjani MN, Achutha V, Khasim DI. Parameters affecting the viscosity of chitosan from prawn waste. Journal of Food Science and Technology. 1975;12:187-189

[30] Wu ACM, Bough WA. A study of variables in the chitosan manufacturing process in relation to molecular-weight distribution, chemical characteristics and waste-treatment effectiveness. In: Proceedings of the 1st International Conference on Chitin/Chitosan, Boston, USA. 1977

[31] Bough WA, Salter WL, Wu ACM, Perkins BE. Influence of manufacturing variables on the characteristics and effectiveness of chitosan products 1 . Chemical composition, viscosity, and molecular-weight distribution of 
chitosan products. Biotechnology and Bioengineering. 1978;20:1931-1943

[32] Sluyanarayana Rao SV, Yashodha KP, Mahendrakar NS, Puttarajappa P. Deacetylation of chitin at low temperature by a novel alkali impregnation technique. Indian. Journal of Technology. 1987;25:194-196

[33] Mima S, Miya M, Iwamoto R, Yoshikawa S. Highly desacetylated chitosan and its properties. Journal of Applied Polymer Science. 1983;28: 1909-1917

[34] Shimahara K, Ohkouchi K, Ikeda M. In: Roberts GAF, editor. Chitin Chemistry. London, UK: Macmillan Press; 1992. p. 56

[35] Younes I, Rinaudo M. Review chitin and chitosan preparation from marine sources. Structure, properties and applications. Marine Drugs. 2015;13: 1133-1174

[36] Broussignac P. Un haut polymère naturel peu connu dans l'industrie, Le chitosane. Chimie and Industrie Genie Chimique. 1968;99:1241-1247

[37] Hakman RH, Goldberg M. Lightscattering and infrared-

spectrophotometric studies of chitin and chitin derivatives. Carbohydrate

Research. 1974;38:35-45

[38] BeMiller JN, Whistler RL. Alkaline degradation of amino sugars. The Journal of Organic Chemistry. 1963;27: 1161-1164

[39] Hakman RH, Goldberg M. Lightscattering and infraredspectrophotometric studies of chitin and chitin derivatives. Carbohydrate Research. 1974;38:35-45

[40] Blumberg R, Southall CL, van Rensburg NJ, Volckman OB. South African fish products. XXXII-The rock lobster: A study of chitin production from processing wastes. Journal of the Science of Food and Agriculture. 1951;2: 571-576

[41] Muzzarelli RAA, Priser ER, editors. Mit Sea Grant Program. MA, USA: Cambridge; 1978. pp. 54-63

[42] Anderson GG, de Pablo N, Romo C. Antartic krill (Euphausia superba) as a source of chitin and chitosan. In: Proceedings of First International Conference on Chitin and Chitosan; 2001

[43] Brzeski MM. Concept of chitin chitosan isolation from Antartic krill (Euphausia superba) shells on a technical scale. In: Hirano S, Tokura S, editors. Proceedings of the Second International Conference on Chitin and Chitosan. Sapporo, Japan: The Japan Society of Chitin and Chitosan; 1982. pp. 15-29

[44] Tolaimate A, Desbrieres J, Rhazi M, Alagui A. Contribution to the preparation of chitins and chitosans with controlled physico-chemical properties. Polymer. 2003;44:7939-7952

[45] Roberts GAF. Structure of chitin and chitosan. In: Roberts GAE, editor. Chitin Chemistry. London, UK: Palgrave Macmillan; 1992. pp. 85-91

[46] Kurita K, Sannan T, Iwakura Y. Studies on chitin, 4: Evidence for formation of block and random copolymers of $\mathrm{N}$-acetyl-D-glucosamine and D-glucosamine by hetero- and homogeneous hydrolyses.

Makromolekulare Chemie. 1977;178: 3197-3202

[47] No HK, Meyers SP. Preparation and characterization of chitin and chitosan -A review. Journal of Aquatic Food Product Technology. 1995;2:27-52

[48] Kafetzopoulos D, Martinou A, Bouriotis V. Bioconversion of chitin to chitosan: Purification and characterization of chitin deacetylase 
from Mucor rouxii. Proceedings of the National Academy of Sciences of the United States of America. 1993;90: 2564-2568

[49] Aiba SI. Preparation of Nacetylchitooligosaccharides by hydrolysis of chitosan with chitinase followed by $\mathrm{N}$-acetylation. Carbohydrate Research. 1994;265: 323-328

[50] Hajji S, Younes I, Ghorbel-Bellaaj O, Hajji R, Rinaudo M, Nasri M, et al. Structural differences between chitin and chitosan extracted from three different marine sources. International Journal of Biological Macromolecules. 2014;65:298-306

[51] Chang KLB, Tsai G, Lee J, Fu WR. Heterogeneous N-desacetylation of chitin in alkaline solution. Carbohydrate Research. 1997;303:327-332

[52] Sannan T, Kurita K, Iwakura Y. Studies on chitin, 2. Effect of desacetylation on solubility. Makromol Chem. 1976;177:3589-3600

[53] Van de Velde K, Kiekens P. Struc ture analysis and degree of substitution of chitin, chitosan and dibutyrylchitin $b$ y FT-IR spectroscopy and solid state 13C NMR. Carbohydrate Polymers. 2004;58: 409-416

[54] Chen R, Wang X, Yao X, Zheng X, Wang J, Jiang X. Near-IR-triggered $h$ otothermal/photodynamic dual-moda lity therapy system via chitosan hybrid nanospheres. Biomaterials. 2013;34(33): 8314-8322

[55] Tolaimate, A. Exploration des gisements chitineux de la faune marine marocaine. Procédé d'extraction de chitines fortement acétylées. Préparation de chitosanes à caractéristiques contrôlées. [Ph.D. Dissertation] Marrakech, Maroc: Cadi Ayyad University; 2000
[56] Tsigos I, Martinou A,

Kafetzopoulos D, Bouriotis V. Chitin deacetylases: New, versatile tools in biotechnology. Trends in Biotechnology. 2000;18:305-312

[57] Araki Y, Ito E. A pathway of chitosan formation in Mucor rouxii: Enzymatic desacetylation of chitin. European Journal of Biochemistry. 1975; 189:249-253

[58] Martinou A, Kafetzopoulos D, Bouriotis V. Isolation of chitin deacetylase from Mucor rouxii by immunoaffinity chromatography. Journal of Chromatography. 1993;644: 35-41

[59] Sundara RG, Aruchami M, Gowri N. Natural desacetylation of chitin to chitosan in the abdominal cuticle of the physogastric queen of Macrotermes estherae. In: Tokura S, Hirano S, editors. Proceeding Second International Conference Chitin/Chitosan, Sapporo, Japan, 12-14 July 1982. Tottori, Japan: Japanese Soc. Chitin; 1982

[60] Gao XD, Katsumoto T, Onodera K. Purification and characterization of chitin deacetylase from Absidia coerulea. Journal of Biochemistry. 1995;117: 257-263

[61] Younes I, Nasri R, Bkahiria I, Jellouli K, Nasri M. New proteases extracted from red scorpionfish (Scorpaena scrofa) viscera: Characterization and application as a detergent additive and for shrimp waste deproteinization. Food and Bioproducts Processing. 2014;94:453-462. DOI: 10.1016/j.fbp.2014.06.003

[62] Kaur S, Dhillon GS. Recent trends in biological extraction of chitin from marine shell wastes: A review. Critical Reviews in Biotechnology. 2015;35: 44-61

[63] Boukhlifi F, El Akili C, Moussout H, Benzakour A, Ahlafi H. Treatment of 
global rejection of electroplating industry by raw chitin. International Journal of Applied Environmental Sciences. 2013;8:13-23

[64] Tsigos I, Bouriotis V. Purification and characterization of chitin deacetylase from Colletotrichum lindemuthianum. The Journal of Biological Chemistry. 1995;270: 26286-26291

[65] Tokuyasu K, Kameyama MO, Hiyashi K. Purification and characterization of extracellular chitin deacetylase from Colletotrichum lindemuthianum. Bioscience, Biotechnology, and Biochemistry. 1996; 60:1598-1603

[66] Baxter A, Dillon M, Taylor KDA, Roberts GAF. Improved method for IR determination of the degree of $\mathrm{N}$ acetylation of chitosan. International Journal of Biological Macromolecules. 1992;14(3):166-169

[67] Brugnerotto J, Lizardi J, Goycoolea FM, Arguelles-Monal W, Desbrieres J, Rinaudo M. An infrared investigation in relation with chitin and chitosan characterization. Polymer. 2001;42(8):3569-3580

[68] Duarte ML, Ferreira MC, Marvao MR, Rocha J. An optimised method to determine the degree of acetylation of chitin and chitosan by FTIR spectroscopy. International Journal of Biological Macromolecules. 2002;31(1-3):1-8

[69] Khan TA, Peh KK, Ch'ng HS. Reporting degree of desacetylation values of chitosan: The influence of analytical methods. Journal of Pharmacy and Pharmaceutical Sciences. 2002;5(3): 205-212

[70] ELOUAHLI Abdelaziz Préparation et caractérisation de poudre pure de phosphate tricalcique. Application à la mise au point d'un nouveau nano- composite phosphate tricalcique apatitique/chitosane pour usage orthopédique et dentaire; 2017. THESE Présentée A Universite chouaib doukkali Faculte des sciences El jadida

[71] Kasaai MR. A review of several reported procedures to determine the degree of $\mathrm{N}$-acetylation for chitin and chitosan using infrared spectroscopy. Carbohydrate Polymers. 2008;71: 497-508

[72] Ren D, Yi H, Wang W, Xiaojun M. The enzymatic degradation and swelling properties of chitosan matrices with different degrees of $\mathrm{N}$-acetylation. Carbohydrate Research. 2005;340: 2403-2410

[73] Dash M, Chiellini F, Fernandez EG, Piras AM, Chiellini E. Statistical approach to the spectroscopic determination of the desacetylation degree of chitins and chitosans. Carbohydrate Polymers. 2011;86:65-71

[74] Miya M, Iwamoto R, Mima S. FTIR study of intermolecular interactions in polymer blends. Journal of Polymer Science. 1984;22:1149-1151

[75] Fenton DM, Eveleigh DE. Purification and mode of action of a chitosanase from Penicillium islandicum. Journal of General Microbiology. 1981; 126:151-165

[76] Wu ACM. Determination of molecular-weight distribution of chitosan by high-performance liquid chromatography. Methods in Enzymology. 1988;161:447-452

[77] Tolaimate A, Desbrieres J, Rhazi M, Alagui A. Contribution to the preparation of chitins and chitosans with controlled physico-chemical properties. Polymer. 2003;44:7939-7952

[78] El-Sherbiny IM. Synthesis, characte rization and metal uptake capacity of a 
new carboxymethyl chitosan derivative. European Polymer Journal. 2009;45:

199-210

[79] He X, Li K, Xing R, Liu S, Hu L, Li P. The production of fully desacetylated chitosan by compression method.

Egyptian Journal of Aquatic Research. 2016;42:75-81

[80] Titik D, Susanto H, Rokhati N.

Influence of microwave irradiation on extraction of chitosan from shrimp.

Shell Waste Reaktor. 2018;18(1):45-50

[81] Samar M, Khaloufi M.

Physicochemical, functional antioxidant and ntibacterial properties of chitosan extracted from shrimp wastes by microwave technique. Annals of Agricultural Science. 2013;500(1):33-41

[82] Horowitz ST, Roseman S, Blumental HJ. Preparation of glucosamine oligosaccharides. 1. Separation. Journal of the American Chemical Society. 1957;79:5046-5049 



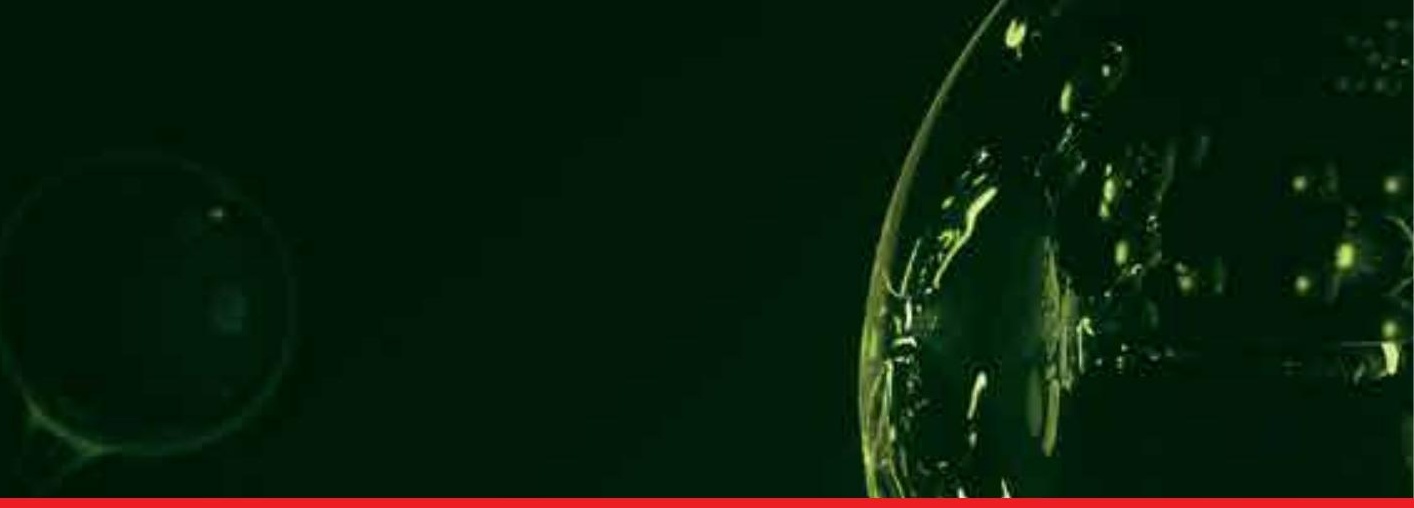

\section{Edited by Maaz Khan, Gustavo Morari do Nascimento and Marwa El-Azazy}

Modern spectroscopic techniques have a number of applications in many fields including material science, physics, chemistry, biology, and medicine. This book, "Modern Spectroscopic Techniques and Applications", presents knowledge about these techniques and their applications. The chapters cover many aspects such as an introduction to atomic microscopy, Raman spectroscopy, infrared spectroscopy and their applications covering both the experimental and theoretical aspects. This book is aimed to provide understanding about modern spectroscopic techniques and their applications to students, scientists, and engineers working in the relevant areas. 